\title{
TANDFM MIRROR REACTOR WITH THERMAL BARRIERS
}

G. A. Carlson, B. Arfin, W. L. Barr, B. M. Boghosian,

J. L. Erickson, J. H. Fink, G. W. Hamilton, B. G.

Logan, J. O. Myall, and W. S. Neef, Jr.

September 19, 1979

Work performed under the auspices of the U.S. Department of

Energy by the UCLLL under contract number W-7405-ENG-48.

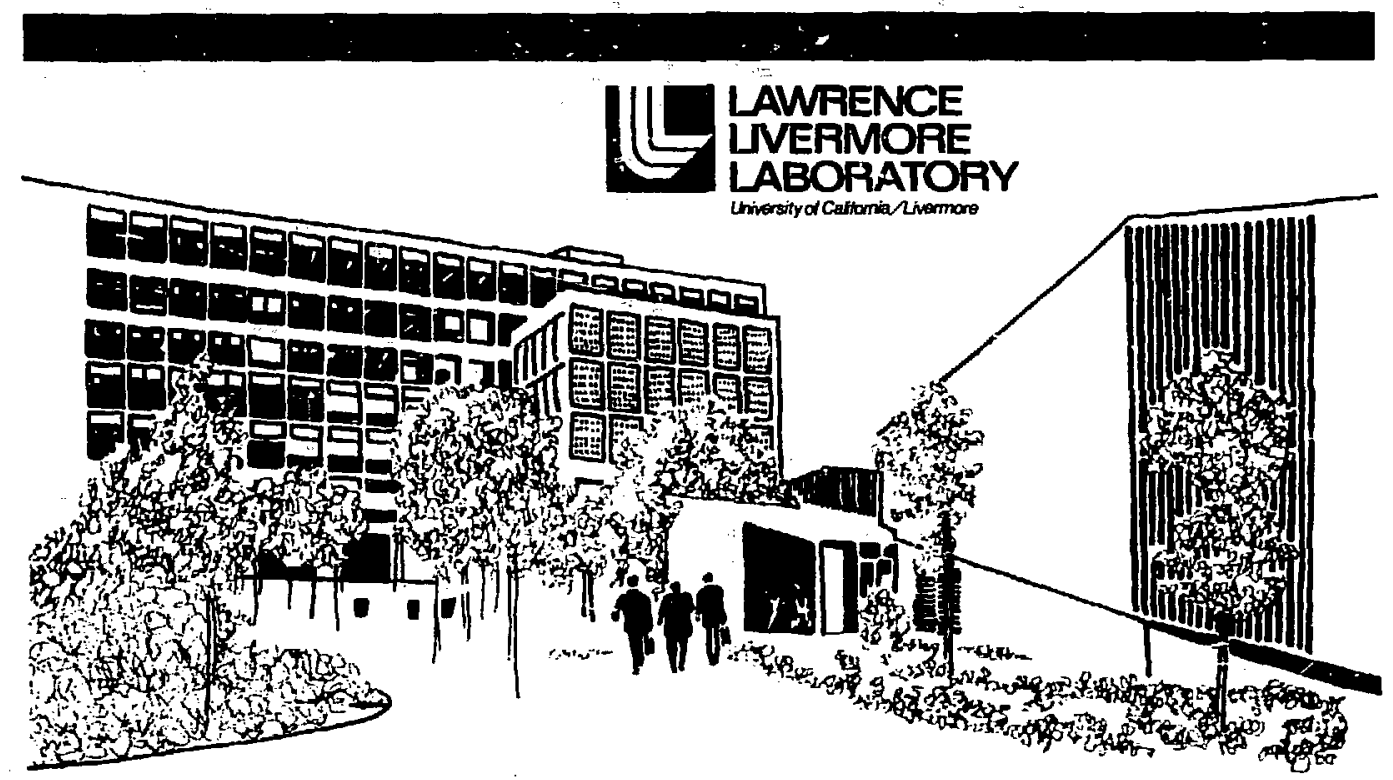

$+$ 


\section{แ4}

\section{LAWRENCE IIVERMORE I_ABORATORY}

University of Caltornia Livermore,California 94550

\section{MASTER}

UCRL-52836

\section{TANDEM MIRROR REACTOR WITH THERMAL BARRIERS}

G. A. Carlson, B. Artin, W. L. Barr, B. M. Boghosian, J. L. Erickson, ${ }^{*}$ J. H. Fink, ${ }^{\dagger}$ G. W. Hamilton, B. G. Logan, J. O. Myall, and W. S. Neef, Jr.

Manuscripl date: September 19, 1979

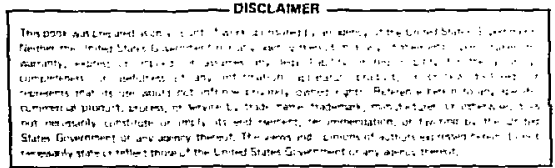

*Grummar Aerospace Corporation.

${ }^{+}$Westinghouse Electric Corporation. 


\section{CONTENTS}

Abstract . . . . . . . . . . . . . . . 1

Chapter 1. Overview . . . . . . . . . . . . 3 (G. A. Carlson)

Chapter 2. Plasma Physics . . . . . . . . . 27 (B. G. Logan, B. M. Boghosian)

Chapcer 3. Magnets . . . . . . . . . 65 (J. 0. Myal1)

Chapter 4. End-Plug Neutral Beams . . . . . . . . 81 (C. W. Hamilton, J. L. Erickson, J. H. Fink)

Chapter 5. Barrier Pump Neutral Beams ....... . 123 (G. W. Hamilton, B. G. Logan)

Chapter 6. Electron Cyclotron Rescnant Heating . . 149 (G. W. Hamilton, B. Arfin)

Chapter 7. Plasma Direct Converter ........ 163 (W. L. Barr)

Chapter 8. Central Cell . . . . . . . . . 181 (W. S. Neef, Jr.) 


\section{ABSTRACT}

The basic tandem mirror concept entails the improved axial confinement of a long cylindrical fusion plasma within a solenoid by means of strong electrostatic potentials at the ends. In the original tandem mirror concept, the confining electrostatic potential was produced by mirror-confined, end-plug plasmas of considerably higher density than the fusion plasma in the solenoidal center cell. As shown in a conceptual reactor design study completed in 1977, the requirement for a high plasma density plug led to advanced technological requirements for plug magnets and neutral-beam injectors.

A major new invention-the thermal barrier-can result in further improved axial confinement and, at the same time, a reduction in the end-plug technological requirements. Basically, the thermal barrier consists of a region of much reduced magnetic field strength, plasma density, and plasma potential between the solenoidal central cell and the end plug. The depressed positive plasma potential is an electrostatic barrier to electrons and permits the establishment of a hotter electron population in the plugs than in the central cell. The hotter electron population in turn increases the electrostatic confining potential. This report describes the concept of the improved tandem mirror reactor with thermal barriers and the progress we have made toward the conceptual design of such a reactor.

An analytic computer model for the tandem mirror plasma with thermal barriers has been constructed. Included is a detailed treatment of thermal ion trapping in the barrier region and barrier "pumping" by neutral beam charge exchange. Other new computer models were constructed to assess the MHD stability of plasmas contained in the new magnetic configuration. 
Major end region elements of the new design are a magnet system comprised of a 12-T solenoidal barrier coil of niobium tin and niobium titanium plug coils (yin-yang shape) and transition coils, a low current $400 \mathrm{keV}$ negative ion type neutral-beam injection system for the plugs, microwave heating of the plug electrons, a positive ion type neutral-beam system for chargeexchange "pumping" of the barrier region, and a plasma direct converter. The central cell is constructed of axial modules, each $2 \mathrm{~m}$ in length. Each module contains an annulus of helium-cooled blanket assemblies, a shield region, and two niobium titanium solenoidal coils.

Based on preliminary calculations, we set as the goal for the new reactor design a 500-MWe output from a high $Q(\geq 10)$ fusion plasma confined in a central cell about $60 \mathrm{~m}$ in length. Although our progress toward this goal has been significant, additional work must be done to achieve a self-consistent reactor design which satisifes al1 of the known physics constraints. The present magnet design has proved inadequate against the criterion for MHD ba1looning stability at our assumed value for central cell $\beta$. Ongoing work emphasizes the redesign of the magnet system to iricrease the $\beta$ limit for stability. 


\section{CHAPTER 1. OVERVIEW}

G. A. Carlson

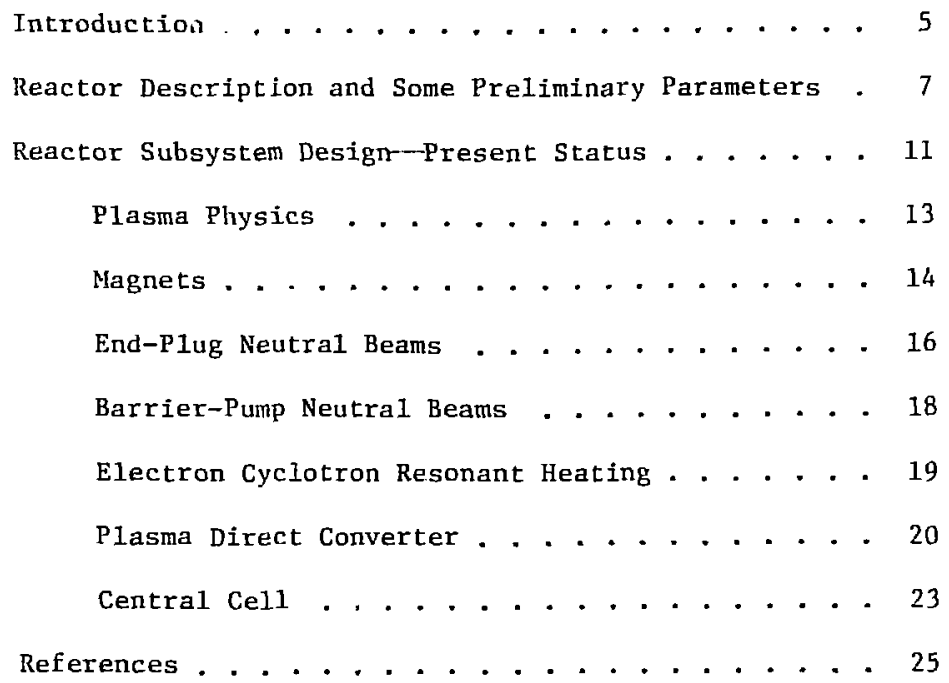


The concept of the tandem mirror magnetic fusion device was invented in 1976 by Logan and Fowler ${ }^{1-1}$ at LLL and independently by Dimov ${ }^{1-2}$ in the USSR. The basic concept entails the improved axial confinement of a long cylindrical Fusion plasma within a solenoid by means of strong electrostatic potentials at the ends, produced by mirror-confined, end-plug plasmas. The first conceptual fusion reactor design based on the concept was published in July $1977^{1-3}$. The reactor had a central cell $100 \mathrm{~m}$ in length and produced 1000 MW of net electrical power from $2800 \mathrm{MW}$ of fusion power. Drawbacks of the first design included a somewhat modest plasma performance $(Q \equiv$ fusion power/total injected power trapped by the plasma $\approx 5$ ) and a requirement for high technology components for the end plugs (17-T magnetic coils and 1.2-MeV neutral-beam injectors).

Work aimed at improving the tandem mirror reactor (TMR) was reported in April 1979. 1-4 In that report we investigated the use of auxiliary plasma cells (A-cells) at the ends of the reactor to improve plug confinement and to reduce the required neutral-beam injection energy, and the use of direct electron heating to further reduce the neutral-beam requirements. These new features also allowed the reduction of the plug magnetic field strengths. One reactor case reported in that study had a central cell $320 \mathrm{~m}$ in length and produced $3000 \mathrm{MW}$ of fusion power. The maximum magnetic field strength (in the conductor of the axisymmetric plug coil set) was $15 \mathrm{~T}$ and the required neutral-beam injection energy was $350 \mathrm{keV}$. The reduced fusion power per metre of central cell length (compared to the 1977 design) was believed to be acceptable because of central cell design simplifications and cost reduction. Also reported in April 1979 was a major new invention for tandem mirrors-the thermal barrier concept. ${ }^{1-5}$ This invention followed from the 
realization that the optimal use of electron heating in the tandem mirror involved the establishment of a hotter electron population in the plugs than in the central cell because the confining electrostatic potential is directly proportional to the electron temperature in the plugs, while cooler central cell electrons allow a higher fusion power density for a given magnetic field strength. However, in the normal tandem mirror, electron flow between the plugs and central cell is so high that only small temperature differences can be established, even with electron heating localized in the end plugs. The new concept introduces a barrier between the plug and central cell that effectively reduces the passing of central cell electrons into the plug. Because of the reduced electron interaction, a modest amount of electron heating in the plugs is sufficient to establish a large temperature difference.

Basically, the thermal barrier consists of a region of much reduced magnetic field strength, plasma density, and plasma potential. The depressed positive plasma potential is an electrostatic barrier to electrons. Although a region of reduced magnetic field strength could be produced in a tandem mirror by simply separating the plug coils from the central cell solenoid, it is more desirable to first increase the magnetic field at the end of the central ce11, and then to produce the low field region by proper spacing of the plug coils. We call the new solenoidal coil at the end of the central cell the barrier coil. The primary physics complication introduced by the thermal barrier concept is the requirement for a method to prevent the filling of the barrier region by thermal ions leaking from the central cell. Such filling is unacceptable because it would negate the depression in plasma potential. In the Apri1 1979 report,,$^{1-4}$ two possible methods for "barrier pumping" were described: heating at the ion bounce frequency and a periodic elimilation of the barrier magnetic well by means of an additional pulsed barrier coil. Since then, a third concept has emerged as the preferred method for barrier 
pumping: the use of neutral beams to charge exchange with the thermal ions trapped in the barrier region. The charge exchange neutrals are lost radially from the plasma. By proper selection of the barrier pump neutral-beam energies and injection angles, the new ions are not trapped in the barrier region, but become fuel ions for the central cell.

In this report we describe the concept of this new TMR with thermal barriers and the progress we have made toward the conceptual design of such a reactor.

REACTOR DESCRIPTION AND SOME PRELIMINARY PARAMETERS

Figure 1-1 shows the proposed design for a 500-Mwe tandem mirror reactor with thermal barriers. The DT fusion plasma is contained in the 56-m-1ong central cell. The central cell consists of 28 2-m-long modules, each containing an annular blanket region, a magnet shield region, and two niobiumLLtanium solenoidal magnets. The entixe central cell resides in a vacuum trench, which allows the module-to-module seal to be made by an annular metal inflatable cushion. When the cushion is deflated, these seals allow the removal of 1ndividual modules by an overhead crane. B1anket coolant gallexies are shown below the vacuum trench.

The end-plug components of the reactor are housed in large cylindrical vacum vessels. Beginning at the central cell end, we see the high field barrier coil (a solenoid), a transition coil to transform the magnetic flux sundle from circular to elliptical, the minimum-B plug coils (a yin-yang pair), and a circularizing coil. The latter coil is used to recircularize the elliptical flux bundle emerging from the yin-yang and permits the use of a circular cross-section direct converter. The end-plug magnets are housed in a 


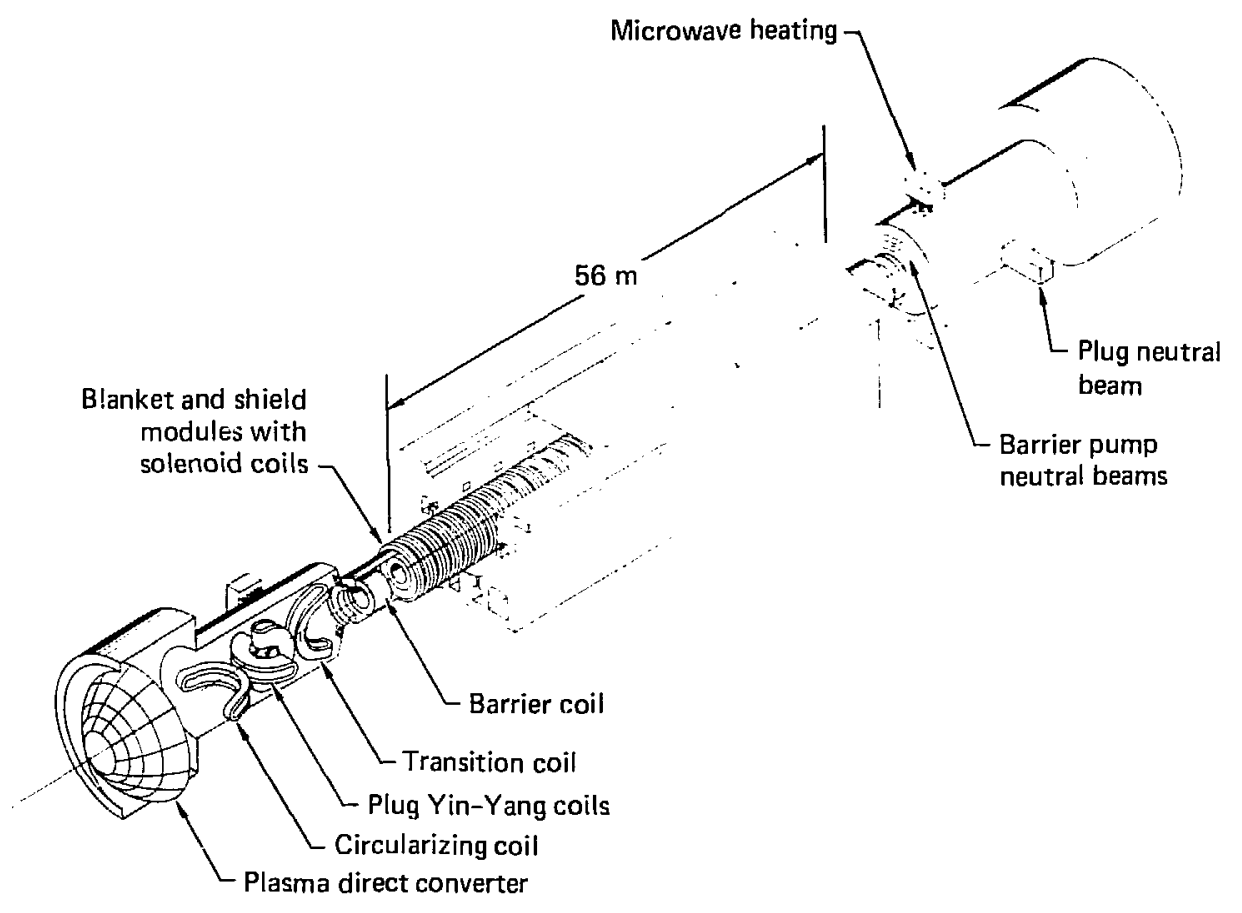

Fig. 1-1. 500-MWe tandem mirror reactor.

12-m-dian vacuum vessel; the plasma direct converter vacuum vessel is $20 \mathrm{~m}$ in diameter.

The plug plasmas, contained in the plug yin-yang coils, are each sustained by a low-current, 400-keV neutral beam (shown only on the far end in Fig. 1-1). Also shown is the gyrotron tube system for microwave heating of the electrons on the piug side of the thermal barrier. The small neutral beams indicated on the end wall of the plug vacuum vessel are the barrier region beams for charge-exchange pumping of the barrier and fueling of the central cell.

The preliminary design shown in Fig. 1-1 was based on a particular magnetic geometry. The various magnetic field strengths along the axis of the 
machine are $2.8 \mathrm{~T}$ in the central cel1, $12 \mathrm{~T}$ at the barrier maximum, $1.2 \mathrm{~T}$ at the barrier minimum, $6 \mathrm{~T}$ at the plug mirrors, and $4 \mathrm{~T}$ at the plug center.

For a central cell plasma $\beta$ of 0.75 , the fusion power is 31 -MW power per metre of central cell length, or $1770 \mathrm{MW}$ for the 56-m-1ong central cell. A preliminary analysis of the injection requirements for this reactor led to the simplified power flow diagram shown in Fig. 1-2. The total injection requirements for both plug regions are 1 MW of plug neutral beams, 54 MW of barrierpump neutral beams, and $108 \mathrm{MW}$ of electron cyclotron resonant heating (ECRH). Dividing the fusion power by the sum of these injection requirements yields a plasma $Q=10.9$. Leaving the central cell are $1420 \mathrm{MW}$ of neutron power and $516 \mathrm{MH}$ of charged particle power. (Here we show the charged particle power as the total injected power plus $20 \%$ of the fusion power, which is an overestimate due to the significant amount of charge-exchange neutral power that will deposit on the wa 1 in the barrier region due to barrier pumping. We roughly compensate for this overestimate by choosing a modest direct conversion efficienc $\xi_{\text {. }}$ ) We have taken the blanket energy multiplication to be 1.2 and have chosen nominal efficiencies of 0.35 for the thermal converter and 0.5 for all of the neutral beams, the ECRH, and the plasma direct converter. As shown in Fig. 1-2, the resulting net electrical power is 527 MWe. Note that the plasma direct converter can provide most of the input power for the neutral beams and FGRH. This is advantageous in that the near instantaneous rise to power possible in the plasma direct converter (as opposed to the risa time of the thermal plant) will allow start-up of the reactor without a large power drain from the utility grid.

Because the central cell plasma is near or at ignition, the power output of the reactor can be increased by increasing the central cel1 length and retaining the same end plug systems. Figure 1-3 shows the resulting power flow diagram for a reactor with a central cell length of $100 \mathrm{~m}$. The fusion power 


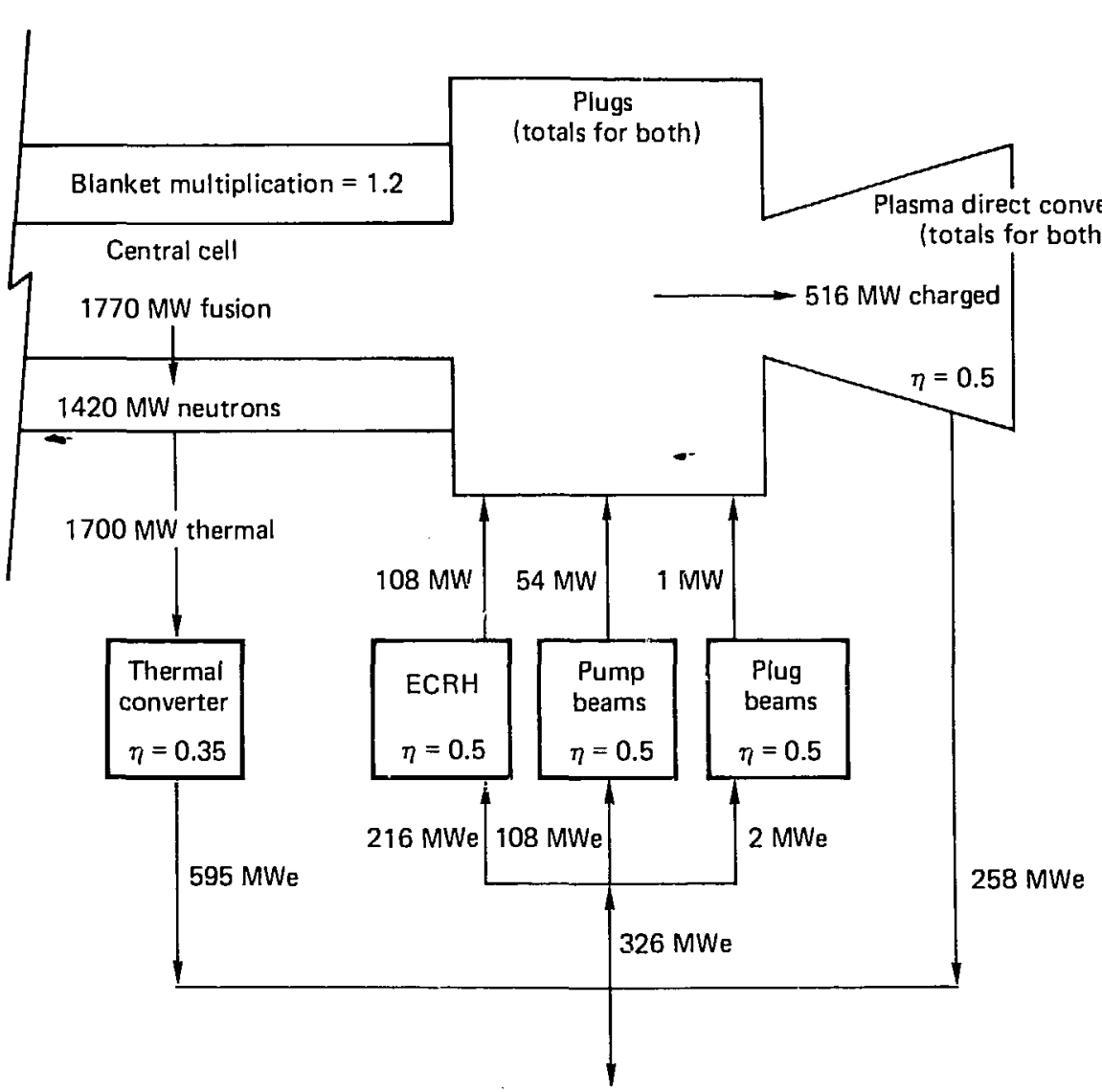

527 MWe net

Fig. 1-2. Power flow diagram for a tandem mirror reactor with 56-m-long central cell $(Q=11)$.

is now $3160 \mathrm{MW}$, and because the end-plug injection requirements have not changed the plasma $Q$ has increased to 19 . The net power output of this 1 arger reactor is 1130 MWe. Note that in this case the plasma direct converter output is more than adequate to power the neutral beams and ECRH.

It must be emphasized that the reactor parameters presented above are based on preliminary analysis and estimates and comprise the goal of our reactor design effort and not, at this time, a final, self-consistent point 


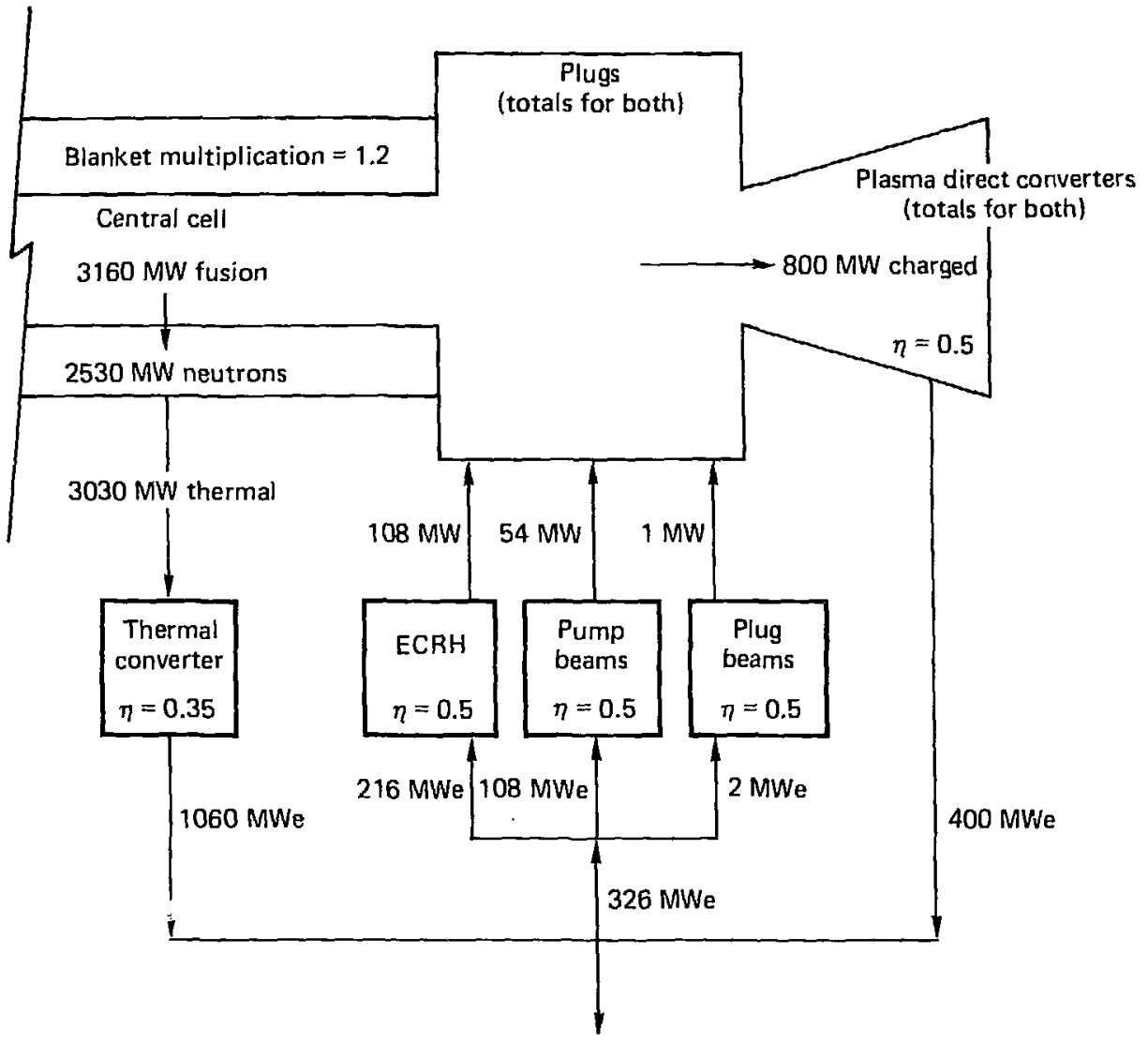

1130 MWe net

Fig. 1-3. Power flow diagram for tanden mirror reactor with $100-m-10 n g$ central cell $(Q=19)$.

design. The following section of this overview and the body of the report describe our progress to date toward that goal.

\section{REACTOR SUBSYSTEM DESIGN-PRESENT STATUS}

In the several months since the invention of the thermal barrier concept, we have made considerable progress toward the design of an improved TMR. Several major new analytic tools have been developed: for example, a 
plasma physics model incorporating a quite detailed treatment of chargeexchange pumped thermal barriers and two computer codes to assess complex tandem mirror magnet designs against plasma magnetohydrodynamic (MHD) stability criteria.

As discussed in the previous section, preliminary estimates of the performance of tandem mirrors with charge-exchange pumped thermal barriers have given us the goal of our design effort. At this time we have not yet achieved that goal. The most obvious shortfall is in the magnet design which failed the criterion for MHD ballooning stability at our assumed value for central cel1 $\beta$ of 0.75 . (This $\beta$ value was demonstrated to more than meet the requirements for MHD interchange stability; unfortunately, the analytic tool to assess the ballooning criterion was not available until after the magnet design was complete.) Also, the large volume of the barrier region contributed to an undesirably large requirement for charge-exchange pumping.

Another contributing factor to the incompleteness of our design is the fact that the new physics model was just very recently completed so as to realistically treat the barrier region, and time has not permitted a thorough exercising of parameter space. Our ongoing work will emphasize the thorough exercising of the physics model and a major redesign of the magnet system. The goal of the magnet redesign will be a substantial shortening of the barrier region that will reduce the charge-exchange pumping requirements and also, we believe, improve the MHD stability situation.

In the remaining sections of the overview, we discuss briefly our progress to date on the individual subsystems of the reactor. Each of these aspects is covered in more detail in the main body of the report. 
The major physics innovation in the present reactor design study is the thermal barrier concept. The thermal barrier consists of a region of much reduced magnetic field strength, plasma density, and plasma potential. The depressed positive plasma potential is an electrostatic barrier to electrons. Because of the reduced interaction of plug and central cell electrons, a.. modest amount of electron heating in the plugs is sufficient to establish an elevated electron temperature there. With thermal barriers the confining potential for central cell ions is given by:

$$
\phi_{c}=T_{e p} \ln \left(\frac{n_{p}}{n_{c}}\right)+\left(T_{e p}-T_{e c}\right) \ln \left(\frac{{ }^{n} c}{n_{b}}\right),
$$

where $\mathrm{T}_{\text {ep }}$ is the plug electr. $\mathrm{n}$ temperature, $\mathrm{T}_{\mathrm{ec}}$ is the central cell electron temperature, $n_{p} / n_{c}$ is the plug-to-central cell density ratio, and $n_{c} / n_{b}$ is the central cell-to-barrier density ratio. It follows that thermal barriers, giving $n_{c} / n_{b}>1$, and ECRH, giving $T_{e p}>T_{e c}$, allow better central cell confinement with lower $n_{p} / n_{c}\left(\right.$ even $\left.n_{p} / n_{c}<1\right)$ and thus a lower magnetic field ratio, ${ }_{\mathrm{p}} / \mathrm{B}_{\mathrm{c}}$.

An analytic model for the tandem mirror plasma with thermal barriers has been constructed and is presented in Chapter 2. Especially noteworthy is the detailed treatment of thermal ion trapping in the barrier region and barrier pumping by neutral-beam charge exchange. Because this part of the physics analysis utilizes magnetic field data, it must be closely coordinated with the magnet design.

Two computer models were constructed to assess the interchange and ballooning modes of MHD stability for plasmas contained in the new tandem mirror 
magnetic configuration. Results from the first model indicate that our present magnet design is satisfactory for the interchange mode. But recent calculations with the second model indicate that the ballooning mode would allow a central cell $\beta$ of only $\sim 0.1$ for our present magnet design. Operation at this low a $\beta$ would result in an unacceptably low plasma $Q$. A preliminary analysis indicates that a considerably shorter barrier region will be needed to meet our high $Q$ reactor goal.

Magnets

The arrangement of magnets is shown in Fig. 1-1, and the field strength variation along the reactor axis has been described. The coil arrangement is also shown in Fig. 1-4. The goal of the design iterations was a magnet arrangement yielding the desired field strength variation, having adequate room For plasma and neutron shielding, and satisfying the MHD criterion for interchange stability at high central cell $\beta$. (The computer code for assessing ballooning stability was not available when the coils were designed.) It was Found that the interchange stability condition was strongly affected by the design of the transition coil, the final version of which is a cee-shaped coil with a $130^{\circ}$ major arc angle. The interchange stability calculation with this transition coil yielded an upper limit of 1.38 for the central cel1-toplug $\beta$ ratio. The transition coil is niobium-titanium superconductor and has a peak conductor field of about $6 \mathrm{~T}$.

The outside diameter of the plug yin-yang pair is $\sim 7.5 \mathrm{~m}$. We wish to use niobium-titanium superconductor for the plug yin-yang coils also, and must therefore limit their maximum conductor field strength to about $8 \mathrm{~T}$. The present design has a maximum field strength of $9 \mathrm{~T}$ in a very localized region at 


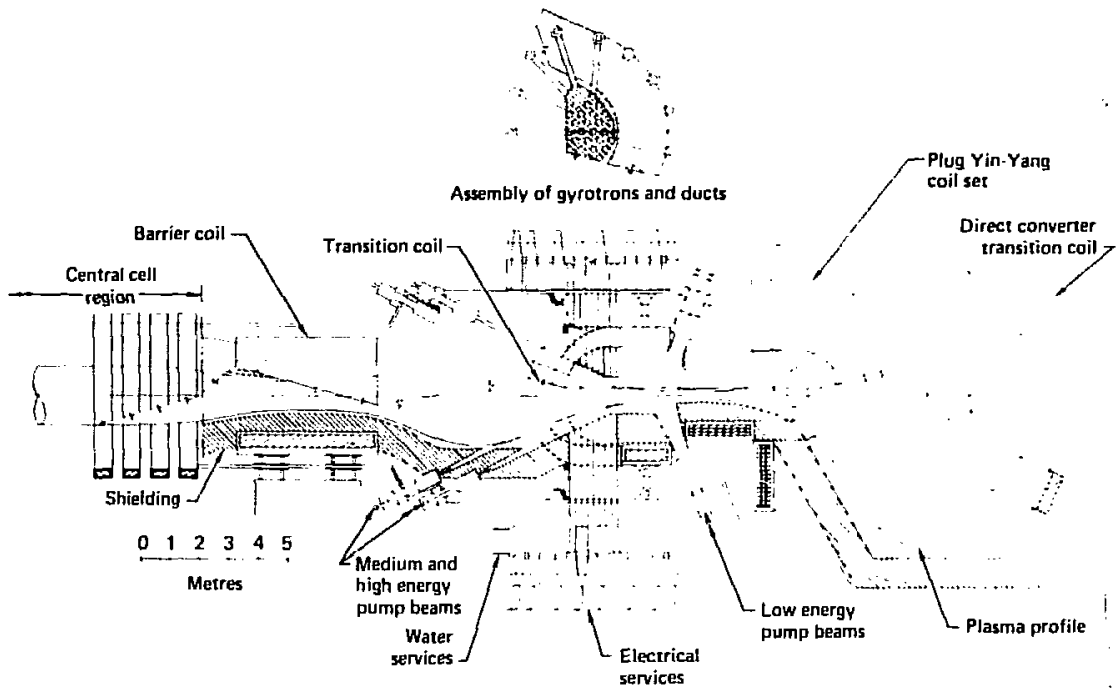

Fig. 1-4. Tandem mirror reactor showing barrier, transition, and plug regions.

the inside radius of the minor arc. A further spreading of the conductors in that region should yield a reduction to $8 \mathrm{~T}$.

The plug barrier coil must be of high magnetic efficiency since we want a 12-T on-axis field and wish to use niobium-tin superconductor presently under development for operation at $212 \mathrm{~T}$. The 4.5-m-1ong solenoid we desigtied has an efficiency of $96 \%$ and, hence, a maximum conductor magnetic field of $12.5 \mathrm{~T}$.

For comparison purposes, we designed an alternative magnet set in which the plug coil was not required to be minimum-B. (In this more speculative 
design the "an=hor" For MHD stability must be provided by some other means, such as a hoi electron ring in the plug, i.e., an EBT cell; we have not yet quantified the stability requirement in such a case.) There is a great simplification of the plug region for such a design. The plug coil is a pair of niobium-titanium solenoids (peak conductor field, $7.9 \mathrm{~T}$ ) only $3.7 \mathrm{~m}$ in outside diameter, and the transition and circularizing cee coils are no longer needed. The plug coil conductor volume is reduced by a factor of almost 5 from the case of the yin-yang pair with two cee coils.

The magnet design is discussed more fully in Chapter 3.

\section{End-Plug Neutral Beams}

The end-plug plasmas are to be sustained by $400-k e V$ neutral-beam injection. We designed a negative ion type neutral-beam injector that produces 1.3 MW of $400-\mathrm{keV}$ neutral $\mathrm{H}^{\circ}$ for injection into the plasma. One injector will be used for each end plug. (Because of beam penetration, only about $0.25 \mathrm{MW}$ of the injected beam will be trapped in the end-plug plasma, the remainder will enter a beam dump.)

An overall view of the injector is shown in Fig. 1-5. The $\mathrm{H}^{-}$ions are produzed by the double charge exchange method. First, $1-\mathrm{keV} \mathrm{H}^{+}$ions are extracted from a steady state positive ion source. About $20 \%$ of these ions are converted to $\mathrm{H}^{-}$by double charge exchange in a cesium vapor cell. Several features of the cell design serve to eliminate cesium loss from the cell: the plug nozzle design, a Elowing eutectic collector at $-20^{\circ} \mathrm{C}$, and venetian blind collectors surrounding the cell apertures. 


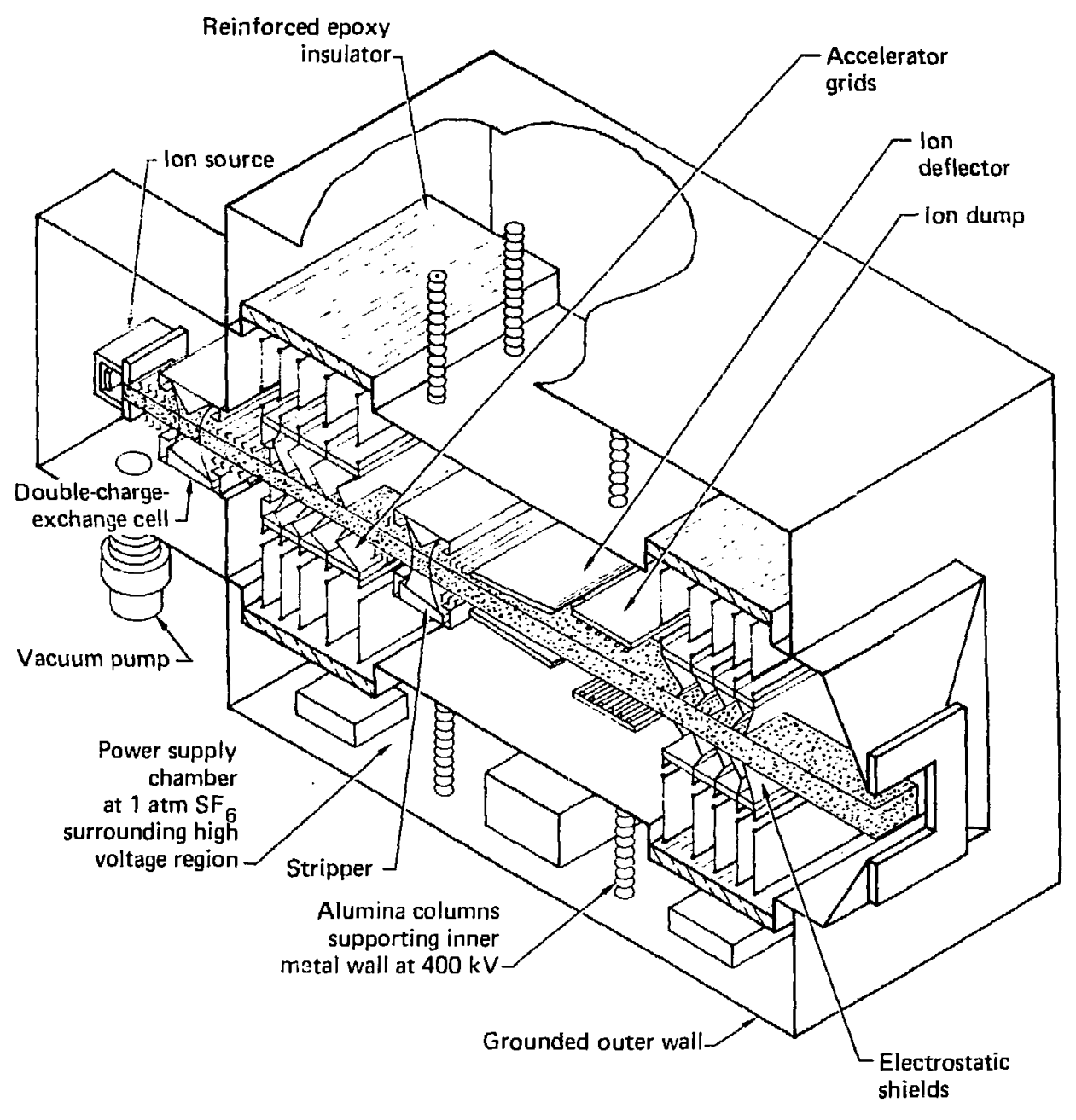

Fig. 1-5. Neutral-beam injector for plug region.

The negative ions are accelerated to $400 \mathrm{keV}$ and then neutralized in a second cesium vapor cell. With line density optimized for maximum electron stripping the neutralization fraction is 0.50 . The remaining ions (about half positive and half negative) are electrostatically deflected onto beam dumps. 
The calculated efficiency for the plug neutral-beam injector is $53 \%$, not including any thermal recovery of beam dump power. Thus the electrical input power requi remelt for each $1.3-\mathrm{MW}$ injector is $2.4 \mathrm{MWe}$.

The plug neutral-beam design is discussed more fully in Chapter 4.

\section{Barrier Pump Neutral Beams}

As discussed previously, we propose the use of neutral beams to charge exchange with the thermal ions trapped in the barrier region and thereby "pump" these ions from the plasma. By proper selection of the barrier pump neutral-beam energies and injection angles, the new ions are not trapped in the barrier region, but become fuel ions for the central cell.

Analysis of the charge-exchange pumping concept has shown that to minimize the power requirements for the pump beams a multistage pumping system is desirable. In this system, decreasing fractions of the total ion load are pumped by injectors of increasing energy level (analogous to a multistage vacuum pumping system in which decreasing fractions of the total gas load are pumped at increasing vacuum levels).

Chapter 5 describes the methodology behind the design of a four-stage barrier pump system. The system consists of a gas jet to charge-exchange with those trapped ions that follow drift surfaces extending out of the main plasma column, and neutral beams at three energies: 10, 50, and $139 \mathrm{keV}$. The neutral beams are a11 of the positive ion type. The locations of the low-, medium-, and high-energy neutral beams are shown in Fig. 1-4.

The specific system described in Chapter 5 was designed to continuously pump a total of $1076 \mathrm{~A}$ of trapped ions from the two barrier regions of the reactor. This trapping rate was the result of a preliminary calculation for 
the magnet system already described and is subject to change with future magnet and plasma iterations. The total neutral currents injected into the barrier region were calculated to be $615 \mathrm{~A}$ for the gas $\mathrm{jet}$, and 697,639 , and 156 A of low-, medium-, and high-energy neutral beam, respectively. This gives a total injected current of $2107 \mathrm{~A}$, nearly double the required pumping rate, largely because of the competing ionization reactions. Multiplying the currents by the beam energies gives the injected power for the pump beams, which totals 60.6 MW for all of the beams. An analysis of the performance of positive ion injectors with no energy recovery for the residual ions exiting the neutralizer yielded a total input power requirement of $119 \mathrm{MWe}$; thus the overall pump beam system efficiency is 0.51 . Note that both the efficiency and the total pump beam power are very close to those in our preliminary parameter set (Figs. 1-2 and 1-3).

\section{Electron Cyclotron Resonant Heating}

Microwave heating of the electrons on the plug side of the thermal barrier is used to maintain the plug electron temperature at about $200 \mathrm{keV}$. The high plug electron temperature has two beneficial effects: it increases the plug potential and thereby the central cell ion confinement and it decreases the electron drag in the plug. The latter effect is responsible for the much reduced current and power level of the plug neutral-beam injectors.

We propose to provide the microwave power by electron cyclotron resonant heating (ECRH). Prime reasons for this choice are (1) that ECRH power can be transmitted by simple waveguides, and (2) that high power oscillators (gyrotrons) in the ECRH frequency range are now becoming available. 
The ECRH power is required to balance two principal electron energy losses in the plug and transition region: (1) conduction energy transfer to those central cell electrons that pass through the thermal barrier, and (2) the introduction of cold electrons by the barrier pump neutral beams. The latter loss dominates and for the barrier pump design discussed above (and in Chapter 5) amounts to a total of $60 \mathrm{MW}$ for both ends of the reactor. The conduction loss is estimated to be $18 \mathrm{MW}$. Thus, $78 \mathrm{MW}$ of absorbed ECRH is required. Fortunately, as shown in Chapter 6, the absorption of ECRH power in our plasma should be essentially 100\%. Thus the total injected ECRH power is $78 \mathrm{MW}$ for the case considered. Note that this is somewhat less that our preliminary estimate (Figs. 1-2 and 1-3).

There is some flexibility in the choice of ECRH frequency. The frequency should be at the electron cyclotron frequency ( $\mathrm{f}_{\text {ce }}=28 \mathrm{~B}$ in units of GHz and T), but must be greater than the electron plasma frequency to avoid reflections from the plasma ( $f_{p e}=8976 \mathrm{n}_{\mathrm{e}}^{1 / 2}$ in units of $\mathrm{Hz}$ and $\mathrm{cm}^{-3}$ ). Fortunately, these two conditions are mutually compatible in the tandem mirror plugs and transition region. We have chosen to introduce the ECRH in the transition region where $B \simeq 2 \mathrm{~T}$ and therefore the required frequency is $=56 \mathrm{GHz}$. The locations of the ECRH gyrotrons and waveguides are shown in Fig, $1-4$.

The ECRH system is discussed in more detail in Chapter 6 .

\section{$\underline{\text { Plasma Direct Converter }}$}

The most striking feature of the plasma direct converter shown in Fig. 1-1 is its circular cross section. This is made possible by the circularizing coil that is placed outside the plug yin-yang. As a result of the 
flux bundle circularization, the maximum dimension of the direct converter vacuum tank is reduced.

The other unusual feature of the plasma direct converter proposed for this reactor is that it handles only the end-loss ions. The ions are preferentially lost at one end of the reactor by a slight lowering of the potential in the one plug. The electrons are preferentially lost at the other end by a relative biasing of the first grid voltages at the two ends. The advantage of this scheme is that the first grid in the plasma direct converter no longer collects all of the electron power. (In the ordinary arrangement the second grid in the direct converter repels the end-loss electrons, which then reflect back and forth through the first grid until they are finally collected.) Thus, the plasma direct converter can be designed for higher ion power density and can be more compact.

Chapter 7 describes the design of a particular direct converter that handles $180 \mathrm{MW}$ of ion power. Another $205 \mathrm{MW}$ of charged particle power exits at the other end of the reactor (via electrons and alpha particles) and is collected on a thermal dump. Note that the total charged particle power is $385 \mathrm{MW}, 131 \mathrm{MW}$ lass than shown on our preliminary power flow diagram, Fig. 1-2. The values used in the direct converter design are from a p1asma case with fusion power and $Q$ nearly the same as Fig. 1-2, and the difference between the charged particle powers is due to the charge exchange neutral power to the wall.

The plasma direct converter has a single collector stage and two watercooled grids and is divided into seven modules-a center module of hexagonal cross section (see Fig. 1-6) and six surrounding modules of trapezoidal shape. It is designed to handle an incident ion $f l u x$ of $280 \mathrm{~W} / \mathrm{cm}^{2}$ and has a maximum diameter (point to point) of about $13 \mathrm{~m}$. It will fit easily into the 20-m-diam vacuum vessel shown in Fig. 1-l, and probably into a smaller vessel. 


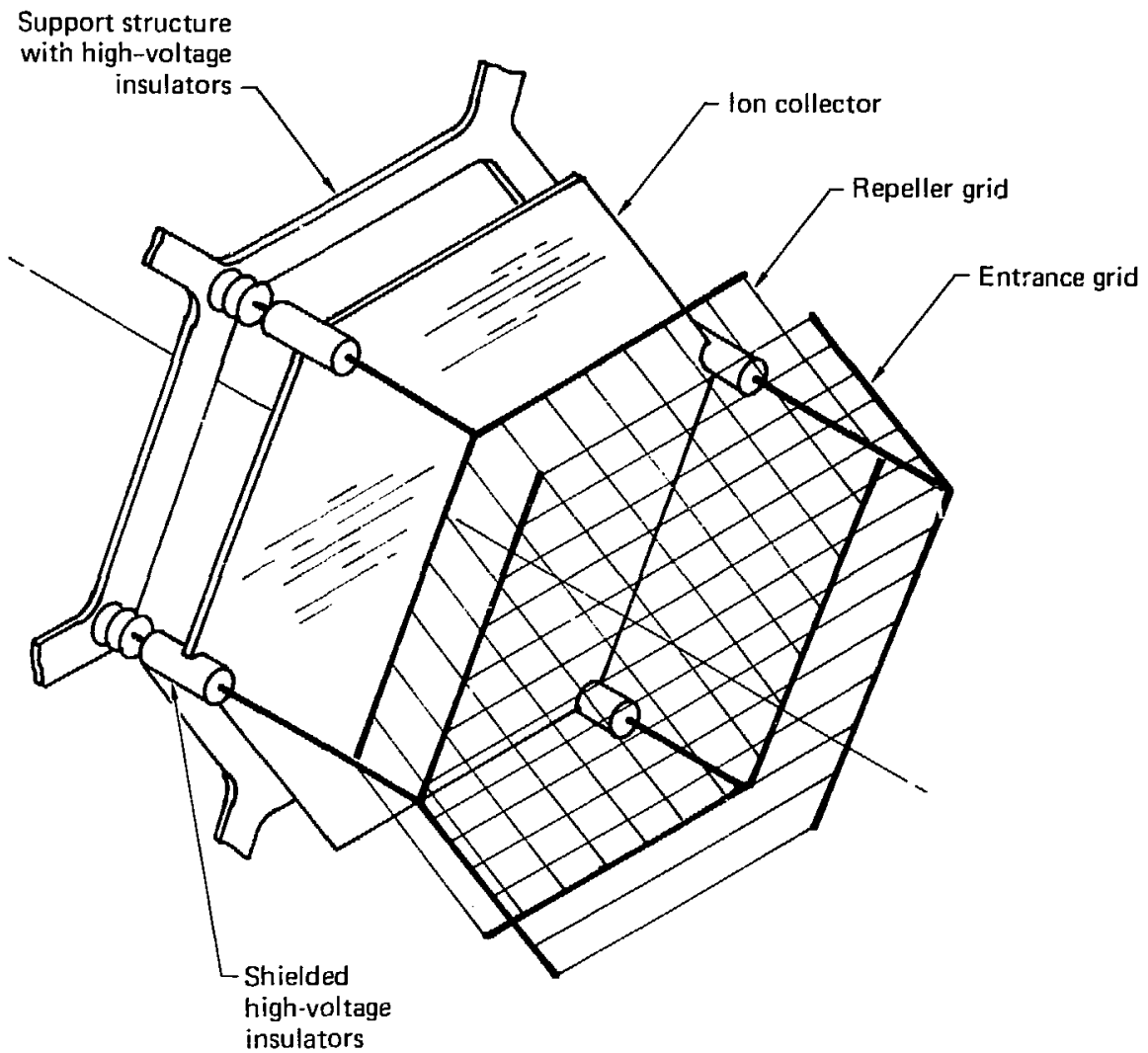

Fig. 1-6. Central module of plasma direct converter.

The calculated electrical output of the direct converter is 115 MHe; thus its operating efficiency is $64 \%$. The electrical output is c insiderably less than estimated in the preliminary power flow diagram (Fig. 1-2) because of the smaller total charged particle power and because of the large electron and alpha power that is deposited on the thermal dump at the other end of the reactor. Note that if this power (205 MW) as well as the collector thermal power in the plasma direct converter ( $47 \mathrm{MW})$ can be thermally converted at an 
efficiency of $35 \%$, the total electrical production from the charged-parti=le end loss is $203 \mathrm{MWe}$, which is only $\sim 50$ MWe short of that estimated in Fig. 1-2.

\section{Centra1 Ce11}

The design goal for the central cell is that it be compact, costeffective, and easily maintained. Figure 1-7 shows three adjacent central cell modules, each $2 \mathrm{~m}$ in length. Each module contains an annulus of blanket pod assemblies, a shield region, and two niobium-titanium solenoid coils.

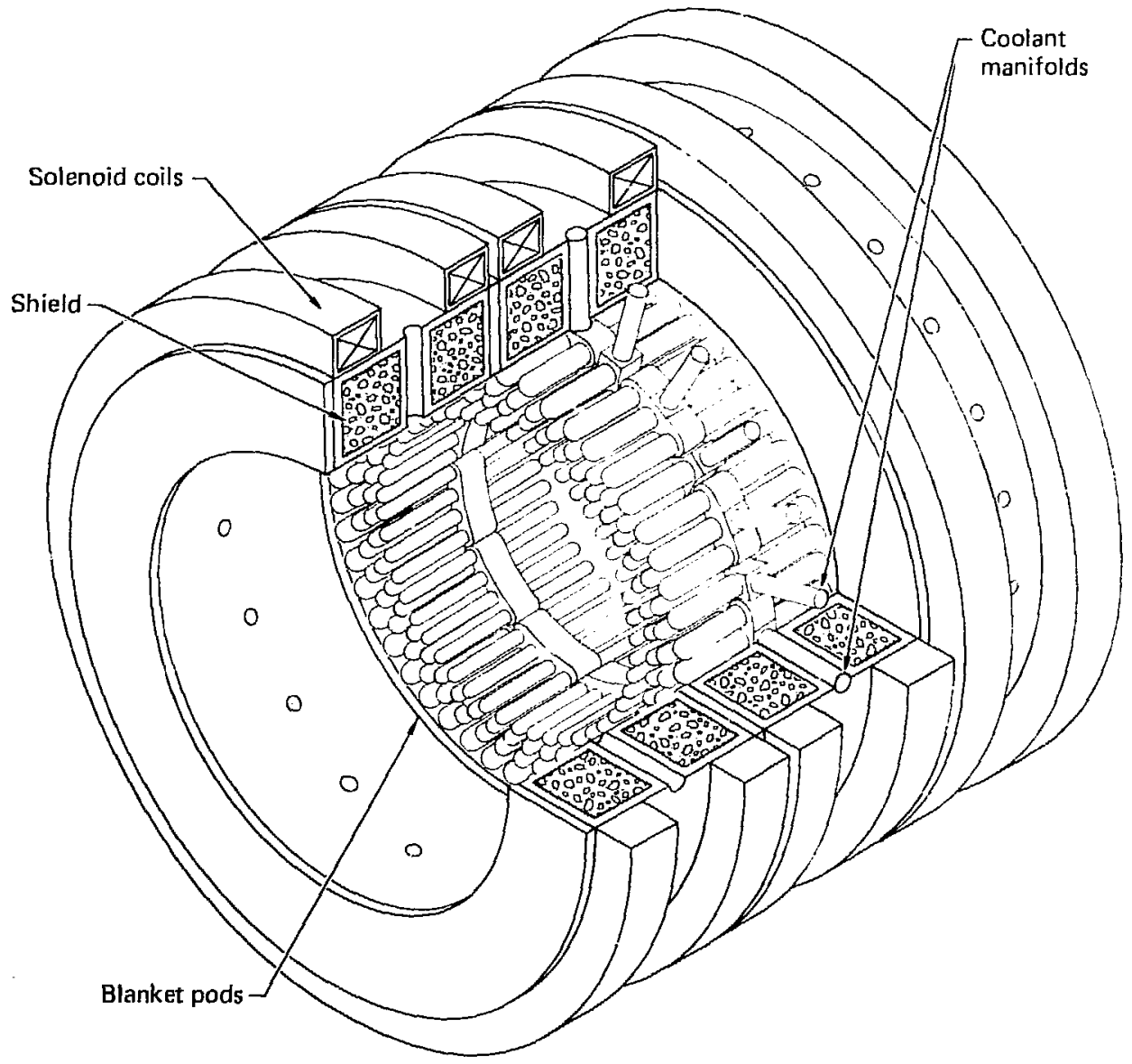

Fig. 1-7. Three central cell modules. 
The blanket pod assemblies are arrangements of cylindrical pods mounted in coolant distributor housings. The pods contain stainless steel canisters of granulated $\mathrm{Li}_{2} \mathrm{O}$ for neutron attenuation and tritium breeding. The can. isters, pod shelis, and coolant distributor housings are all helium-cooled. The coolant inlet and outlet pipes are contained in the stems protruding through the shield from the pod assemblies. The shield is made of poured lead concrete inside a steel case.

A three-dimensional analytic model of the blanket pod assembly has been constructed for use with the Monte Carlo neutronic code TARTNP. Our neutronic calculations predict a tritium breeding ratio of over 1.1 and a blanket neutron-energy multiplication factor of about 1.05 . Note that the latter value is somewhat lower than that estimated in Figs. 1-2 and 1-3.

As described previously, the central cell is housed in a vacuum trench, and intermodule seals are made by annular metal inflatable cushions in the shield region. For blanket changeout or other major maintenance tive 500tonne, central cell modules can be extracted by an overhead crane. For the repair of local blanket failures we have designed the pod assemblies so that they can be removed from inside the central cell without extracting the entire module. The defective pod assembly is removed by a remotely operated service car that travels in the bore of the central cel1.

The central cell design is presented in more detail in chapter 8. 
1-1. T.K. Fowler and B.G. Logan, "The Tandem Mirror Reactor," Comments Plasma Phys. 2, $167(1977)$.

1-2. G.I. Dimov, V.V. Zakaidakov, and M.E. Kishinevsky, "Open Trap With Ambipolar Mirrors," Fiz. Plasmy 2, 597 (1976).

1-3. R.W. Moir, W. L. Barr, G. A. Carlson, W. L. Dexter, J. N. Doggett, J. H. Fink, G. W. Hamilton, J. E. Lee, B. G. Logan, W. S. Neef, Jr., M. A. Peterson, and M. E. Rensink, Preliminary Design Study of the Tanden Mirror Reactor, Lawrence Livermore Laboratory, Livermore, CA, JCRL-52302 (1977).

1-4. G.A. Carlson, B. M. Boghosian, J, H. Fink, J. 0. Myal1, and W. S. Neef, Jr., Parametric Studies of Tandem Mirzor Reactors, Lawrence Livermore Laboratory, Livermore, CA, UCID-18158 (1979).

1-5. D.E. Baldwin, B.G. Logan, and T.K. Fowler, An Improved Tandem Mirror Fusion Reactor, Lawrence Livermore Laboratory, Livermore, CA, UCID-18156 (1979). 


\section{CHAPTER 2. PLASMA PHYSICS}

B. G. Logan, B. M. Boghosian

Introduction . . . . . . . . . . . . . . . 29

Description of the Plasma Mode1.. . . . . . . . 35

Definition and Nomenclature, . . . . . . . . 35

Determination of Potentials and

Densities in the Thermal Barrier . . . . . . . 39

Trapped Ion Pumping in the Thermal Barrier . . . 43

Plug Model . . . . . . . . . . . . . 48

The Particle and Energy Balance Equations . . . 51

TMR Scaling With Thermal Barriers . . . . . . . 57

References .............. 63 
In previous designs for tandem mirror reactors, ${ }^{2-1}$ fusion ions in the center cell were axially confined by electrostatic potentials of end-plug mirror plasmas that were more dense than the plasma in the center ce11-more dense in order to provide a center-cell potential well

$$
\phi_{c}=T_{e} \ln \left(\frac{n_{P}}{n_{c}}\right)
$$

where $n_{p} / n_{c}$ was the plug-to-center-cell density ratio and $T_{e}$ was an assumed axially uniform electron temperature. Since $\phi_{c}$ increases only logarithmically with the density ratio $n_{p} / n_{c}$, whereas the ratio of center-cell fusion power density to the injection power density required to maintain the plugs varies as $\left(n_{c} / n_{p}\right)^{2}$, constraints on the reactor $Q(Q \geq 5)$, power $\left(P_{\text {net }} \lesssim 1000 \mathrm{MWe}\right)$, and neutron wall loading $\left(\Gamma \gtrsim 1 \mathrm{MW} / \mathrm{m}^{2}\right)$ led to fairly stringent technology requirements in the plug magnetic fields $\left(B_{\text {cond }} \gtrsim 17 \mathrm{~T}\right.$ ) and neutral-beam injection energies $\left(E_{i n j} \gtrsim 1 \mathrm{MeV}\right)$. Increasing $\mathrm{T}_{\mathrm{e}}$ by auxiliary heating of the center cell electrons improved the situation somewhat, allowing the neutralbeam energy of the plug to be decreased to about $600 \mathrm{keV}$, while $Q$ was increased to 10 at $\mathrm{P}_{\text {net }}=1000 \mathrm{MW}(\mathrm{e})$ and $\Gamma=0.6 \mathrm{MW} / \mathrm{m}^{2} ;$ however, the required plug magnetic fields were still high $\left(\mathrm{B}_{\text {mirror }}>15 \mathrm{~T}, \mathrm{~B}_{\text {cond }}>20 \mathrm{~T}\right) .^{2-2}$ Recent observations of axial $\mathrm{T}_{\mathrm{e}}$ gradients in the 2 XITB experiment ${ }^{2-3}$ have prompted consideration of the finite power/volume transferred between electrons trapped in the local plug potential well $\Delta \phi$ at temperature $\mathrm{T}_{\text {ep }}$ and transiting center ce 11 electrons at temperature $\mathrm{T}_{\text {ec }}$ with the estimated magnitude

$$
\mathbf{P}_{c p} \approx G_{e} \frac{n_{p}^{2}}{\left(n_{T_{e e}}\right)} \quad\left(T_{e p}-T_{e c}\right) \exp \left(-\frac{\Delta \phi}{T_{e p}}\right),
$$


where

$$
(n \tau)_{\text {ee }}=\frac{8.2 \times 10^{9}}{\ln \Lambda_{\text {ee }}}\left[\mathrm{T}_{\mathrm{ep}}(\mathrm{keV})\right]^{3 / 2} \mathrm{~cm}^{-3} \mathrm{~s}
$$

and $G_{e}$ of order unity is a weak function of potential and mirror ratio. (Accurate determination of this transfer rate is important for detailed reactor calculations and is being pursued by analytical and numerical means.) By applying auxiliary electron heating such as electron cyclotron resonance heating (ECRH) to the plug electrons rather than to the center-cell electrons, and by using Eq. (2-2) with $\Delta \phi=\phi_{c}$ for calculating the increased local plug electron temperature arising from the electron heating power transferred from trapped plug electrons to passing center-cell electrons, we obtained further improvements in neutron wall loading (to $\Gamma \geq 1.2 \mathrm{MW} / \mathrm{m}^{2}$ ) at $Q=10$ and $P_{\text {net }}=1000 \mathrm{MW}(\mathrm{e})$ together with a reduction in peak plug magnetic field to $\mathrm{B}_{\text {cond }}(\max ) \approx 14 \mathrm{~T}^{2-4}$ These further improvements in tandem mirror reactor (TMR) performance obtained by locating the electron heating in the plugs came about because, for $\mathrm{T}_{\text {ep }}$ not too much greater than $\mathrm{T}_{\mathrm{ec}}$, the plug-to-center-cell potential $\psi_{c}$ increases roughly proportionally to the local plug electron temperature; i.e.,

$$
\phi_{c} \approx \mathrm{T}_{\text {ep }} \ln \left(\mathrm{n}_{\mathrm{p}} / \mathrm{n}_{\mathrm{c}}\right),\left(\text { when } \mathrm{n}_{\mathrm{p}}>\mathrm{n}_{\mathrm{c}}\right) \text {. }
$$

Alternatively, for a given required potential well $\phi_{c}$, the plug density $\mathbf{n}_{\mathbf{p}}$ could be reduced if $\mathrm{T}_{\text {ep }}$ were increased by heating.

A new concept called thermal barriers ${ }^{2-5}$ promises to improve the TMR performance much further still by exploiting a greater temperature difference $\mathrm{T}_{\text {ep }}-\mathrm{T}_{\text {ec }}$ made possible by reducing the plug density $\mathrm{n}_{\mathrm{p}}$ even below the center-cell density $n_{c}$. Thermal barriers are localized reductions $1 n$ field, density, and potential between the center cell and the electron-heated plugs (see Fig. 2-1). As center-cell ions pass through the peak field of the 

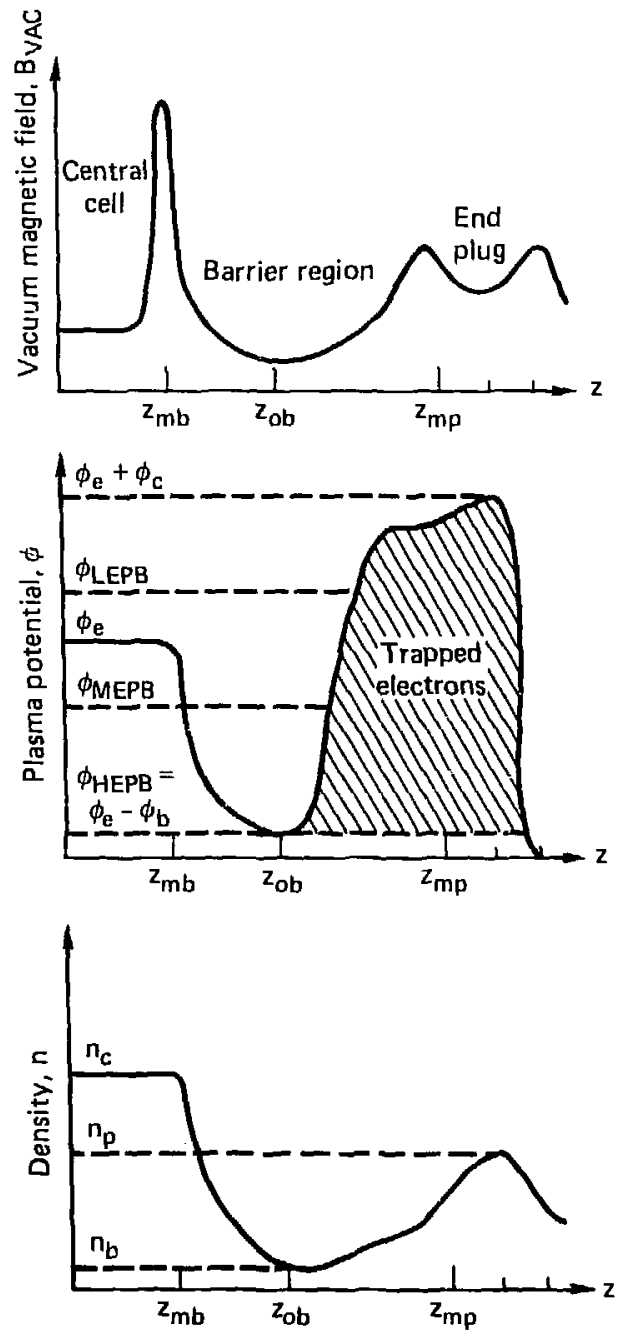

Fig. 2-la. Vacuum magnetic field profile.

Fig. 2-1b. Plasma potential profile.

Fig. 2-1c. Ion density profile. 
barrier mirror (field $\mathrm{B}_{\mathrm{mb}}$ in Fig. 2-1), their density falls with the decrease in field and potential to a minimum

$$
n_{\text {pass }}(b) \approx \frac{n_{c} B_{b}}{B_{m b}}\left(\frac{T_{i c}}{\pi \phi_{b}+T_{i c}}\right)^{1 / 2}
$$

at the minimum field $B_{b}$ in the barrier. Added to the density will be the density of ions trapped by collisions in the barrier region, which must be removed by some means within a collision time of order

$$
\tau_{\text {trap }} \approx \frac{5 \times 10^{11} \mathrm{~T}_{i c}^{3 / 2}}{n_{\text {pass }(b)} \ln \Lambda}\left(\frac{1}{R_{b}}\right)
$$

where $R_{b}=B_{n b} / B_{b}$ is the barrier mirror ratio, and $T_{i c}$ is the temperature of the center-cell ions. If we normalize the total ion density (trapped + passing) to the density of the passing ions,

$$
\mathrm{n}_{\mathrm{b}}=\mathrm{g}_{\mathrm{b}} \mathrm{n}_{\text {pass }}(\mathrm{b}) \text {, }
$$

a potential drop between the center cell and barrier minimum of magnitude

$$
\phi_{b}=T_{e c} \ln \left(\frac{n_{c}}{n_{b}}\right)
$$

occurs when $n_{b}<n_{c}$ due to removal of trapped ions (pumping). Provided $n_{b}$ is also less than ${ }_{p}$, there is a potential rise going from the barrier to the plug given roughly by a formula similar to Eq. (2-4):

$$
\phi_{c}+\phi_{b}=T_{e p} \ln \left(\frac{n_{p}}{n_{b}}\right)
$$

Combining Eqs. (2-8) and (2-9), we obtain

$$
\frac{\mathrm{n}_{\mathrm{p}}}{\mathrm{n}_{\mathrm{c}}} \approx \exp \left(-\frac{\phi_{\mathrm{b}}}{\mathrm{T}_{\mathrm{ec}}}+\frac{\phi_{\mathrm{b}}+\phi_{\mathrm{c}}}{\mathrm{T}_{\mathrm{ep}}}\right) \text {. }
$$


By heating $T_{e p}>T_{e c}\left(1+\phi_{c} / \phi_{b}\right)$, we can achieve a plug potential greater than the center-cell potential by $\phi_{c}$, sufficient to confine the center-cell ions, while the plug density is reduced below that of the center cell $\left(n_{p} / n_{c}<1\right)$. Then the ratio of electron heating power $\approx n_{p}^{2}$ by Eq. $2-2$, using $\Delta \phi=\phi_{c}+\phi_{b}$ with thermal barriers, to the center-cell fusion power $\propto n_{c}^{2}$ is reduced relative to the case without thermal barriers for the same $\phi_{c}$, $T_{\text {ep }}$, and $T_{\text {ec }}$ by a factor of approximately

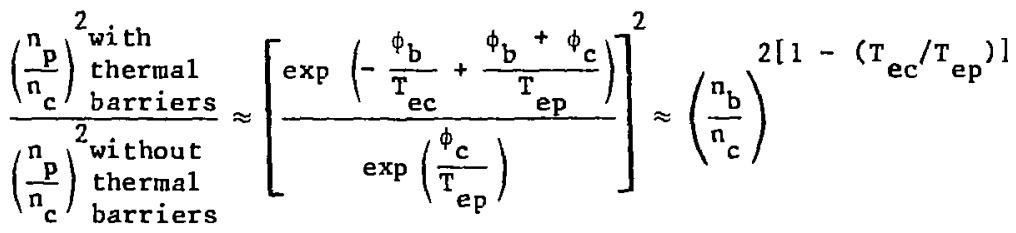

Note that for this factor to be desirably small, we must achieve both $n_{b}<n_{c}$ by pumping trapped ions out of the thermal barrier and also $T_{\text {ep }}>T_{\text {ec }}$ by heating electrons in the plugs, simultaneously.

The TMR reactor $Q$ can be written in a simplified way as

$$
Q \equiv \frac{P_{\text {fusion }} V_{c}}{\left(P_{E C R H}+P_{N B}\right) 2 V_{p}+P_{p u m p} 2 V_{b}} \text {, }
$$

where $P_{\text {fusion }}$ is the fusion power density in the center-cell volume $V_{c}, P_{E C R H}$ and $\mathrm{P}_{\mathrm{NB}}$ are the electron heating and neutral beam heating power densities, respectively, in the plugs of volume $2 \mathrm{v}_{\mathrm{p}}$, and where $\mathrm{P}_{\text {pump }}$ is the power density in the barrier plasma volumes $2 V_{b}$ required to remove the trapped ions:

$$
\mathrm{R}_{\text {pump }} \approx \mathrm{G}_{i} \frac{\mathrm{n}_{\mathrm{b}}}{\tau_{\text {trap }}} \phi_{\mathrm{b}},
$$

where $G_{i}$ is a proportionality constant that depends on the specific pumping mechanism to be used for removing the trapped ions from the barrier. Since 
$P_{N B}$ scales with $n_{P}^{2}$ as well as $P_{C_{P}}$, we can expect $Q$ to improve with the addition of thermal barriers, inversely with the $\left(n_{p} / n_{c}\right)^{2}$ factor given by Eq. (2-11), up to the point at which $P_{\text {pump }}$ becomes the remaining dominant term. In fact, we shall find $\mathrm{P}_{\text {pump }} 2 \mathrm{~V}_{\mathrm{b}} \gg\left(\mathrm{P}_{E C R H}+\mathrm{P}_{\mathrm{NB}}\right) 2 \mathrm{~V}_{\mathrm{p}}$ once $\mathrm{n}_{\mathrm{b}} \ll \mathrm{n}_{c}$ is achieved with thermal barriers. The extent to which the iimiting $Q$ then given by

$$
\mathrm{Q}=\frac{\mathrm{P}_{\text {fusion }}}{\mathrm{P}_{\text {pump }}}\left(\frac{\mathrm{V}_{\mathrm{c}}}{2 \mathrm{~V}_{\mathrm{b}}}\right)
$$

can be greater than $Q$ in previous TMR cases without thermal barriers depends on the efficiency of pumping [e.g., the coefficient $G_{i}$ in Eq. (2-13)] and on the barrier volume $v_{b}$, which depends on the design of the magnetic field. A new pumping scheme based on a combination of charge exchange of trapped ions on neutral beams aimed within the barrier loss cone, together with bad drift orbits of some of the trapped ions ding to their radial loss ${ }^{2-6}$, appears to be the most energy-efficient way of removing the trapped ions, as well as requiring less sophisticated technology than various other pumping schemes discussed in Ref. 2-5. The charge-exchange pumping system is described i: more detail in Chapter 5 of this report.

Besides enhancing $Q$, thermal barriers are also expected to increase neutron wall loading and to reduce plug magnetic fields and injected neutral-beam current and voltage. The basic factor of reduction in $\left(\mathrm{n}_{\mathrm{p}} / \mathrm{n}_{\mathrm{c}}\right)^{2}$ by $\mathrm{Eq} .(2-11)$ for thermal barriers can be exploited two ways. The center-cell density can be increased, increasing fusion power density and correspondingly by the first-wall neutron loading $\Gamma$. A factor of about 4 higher $n_{c}^{2}$ from previous TMR designs should be sufficient to raise $\Gamma$ to maximum economic values set by heat transfer and wall life considerations. Beyond these increases in $n_{c}^{2}$, we can then reduce $n_{p}^{2}$, allowing reductions in confining plug magnetic fields 
$B_{p} \propto \sqrt{n_{p}}$. Once the maximum conductor field $B_{\text {cond }}$ falls below $12 \mathrm{~T}$, further reductions in $B_{p}$ with $n_{p}$ can allow increases in the $p$ lug wirror ratio $R_{p}=B_{\text {mp }} / B_{P}$, which in turn lowers the minimum neutral-beam injection energy set by the minimum cutoff energy

$$
E_{i n j}>E_{\text {cutoff }}=\frac{\phi_{c}+\phi_{e}}{R_{p}-1}
$$

that can be confined by the mirrors against the outer plug potential drop $\phi_{c}+\phi_{e}$. Perhaps the most important technology relaxation made possible by thermal barriers, however, is the reduction in neutral-beam current $\propto n_{p}^{2}$. For given beam access area through the magnets, reductions in total beam current allow reductions in the beam current density $J_{B}$, easing dc beam source design, electric fields in the accel gaps (gap spacing $d \propto 1 / \sqrt{J_{B}}$ ), and gridsputtering lifetimes. Alternatively, lower beam currents would permit smaller individual beam sizes, easing gas pumping, stored electrostatic energy, and beam dump problems. Ultimately, it may be possible to reduce individual beam sizes to less than $1 \mathrm{~A}$ each, where well established accelerator technology such a linacs could be used for voltages above a few hundred keV. Finally, sufficient reductions in neutral-beam power with thermal barriers will relax efficiency and cost/watt requirements.

DESCRIPTION OF THE PLASMA MODEL

\section{Definitions and Nomenclature}

Figures 2-1(a), (b), and (c) display the vacuum magnetic field profile, ambipolar potential profile, and ion density profile at one end of the barrier ce11 TMR. The barrier cell is the region between $z_{b m}$ (barrier mirror) and $z_{p m}$ 
(inboard plug mirror); the transition region is the subset of the barrier cell lying between $z_{b 0}$ (barrier midplane) and $z_{p m}$. The ion density of the central cell is $n_{c}$, that at the barrier midplane is $n_{b}$, and that at the plug midplane is $n_{p}$. The temperature of central-cell ions is $T_{c}$, that of passing electrons is $T_{e c}$, and that of trapped (plug and transition region) electrons is $T_{e p}$. The quantities $n^{*}(z), n_{\text {pass }}(z), B_{\text {vac }}^{*}(z), \phi^{*}(z)$, and $\beta^{*}(z)$ are the total ion density, passing ion density, vacuum magnetic field, ambipolar potential, and perpendicular beta within the barrier-cell region, respective1y. Thus, by definition:

$$
\begin{aligned}
& n^{*}\left(z_{b m}\right)=n_{p a s s}\left(z_{b m}\right)=n_{c}, \\
& n^{*}\left(z_{b 0}\right)=n_{b}, \\
& B_{v a c}^{*}\left(z_{b m}\right)=B_{b m, v a c}, \\
& B_{v a c}^{*}\left(z_{b 0}\right)=B_{b 0, v a c}, \\
& B_{v a c}^{*}\left(z_{p m}\right)=B_{p m, v a c}, \\
& { }^{*}\left(z_{b m}\right)=\phi_{e},
\end{aligned}
$$

and

$$
\phi^{*}\left(z_{b 0}\right)=\phi_{e}-\phi_{b}
$$


The function $g_{b}(z)$ is defined as the ratio of total ion density to passing ion density in the barrier cell:

$$
g_{b}(z)=n^{*}(z) / n_{p a s s}(z)
$$

Note that $\mathrm{g}_{\mathrm{b}}\left(\mathrm{z}_{\mathrm{bm}}\right)=1$ since there can be no trapped ions at the barrier mirror. The quantity

$$
g_{b 0} \equiv g_{b}\left(z_{b 0}\right)>1
$$

is a measure of pumping effectiveness, and approaches 1 from above as the pumping improves (fewer ions trapped in barrier). The following model is used for the $g_{b}(z)$ profile:

$$
g_{b}(z)=1+\left(g_{b 0}-1\right) \quad \frac{\left[1-\frac{B_{v a c}^{*}(z)}{B_{b m, v a c}}\right]}{\left[1-\frac{B_{b 0, v a c}}{B_{b m, v a c}}\right]} ;
$$

that is, $g_{b}$ is assumed to vary linearly with $B_{\text {vac }}^{*}$.

Finally, we note that the plasma magnetic field in the barrier cell is given by

$$
\mathrm{B}_{\mathrm{pla}}^{*}(z)=\mathrm{B}_{\mathrm{vac}}^{*}(z)\left[1-\beta^{*}(z)\right]^{1 / 2}
$$

and that the barrier-ce11 vacuum and plasma mirror ratios are given by

$$
R_{b, v a c} \equiv B_{b m, v a c} / B_{b 0, v a c}
$$


and

$$
\mathrm{R}_{\mathrm{b}} \equiv \mathrm{B}_{\mathrm{bm}, \mathrm{p} 1 \mathrm{a}} / \mathrm{B}_{\mathrm{b} 0, \mathrm{pla}}
$$

respectively.

Other miscellaneous definitions: The energy of alpha particles born in fusion reactions ( $3.52 \mathrm{MeV}$ ) is denoted by $\mathrm{E}_{\alpha}$, and the fractions of this superthermal alpha energy imparted to electrons and ions are given by

$$
\mathrm{f}_{\alpha \mathrm{e}} \approx 0.8816 \exp \left(-\mathrm{T}_{\mathrm{ec}} / 67.40\right),
$$

and

$$
\mathrm{F}_{\alpha i} \approx 1.0-0.9080 \exp \left(-\mathrm{T}_{\mathrm{ec}} / 101.7\right)
$$

respectively. (Note that the two expressions do not add up to unity because of superthermal alphas that scatter into the loss cone while slowing down.) The fusion neutron energy $(14.06 \mathrm{MeV})$ is denoted by $E_{n}$, and the total fusion energy release $(17.58 \mathrm{MeV})$ is denoted by $E_{F U S^{*}}$ The fusion reaction rate averaged over a Maxwe 11 ian plasma is modeled by ${ }^{2-7}$

$$
<\sigma>_{D T} \approx \frac{3.05 \times 10^{-13} \exp \left(-17.7 / \mathrm{T}_{c}^{0.348}\right)}{\left(1.0+0.1554 \mathrm{~T}_{\mathrm{c}}^{1 / 3}-0.1418 \mathrm{~T}_{\mathrm{c}}^{2 / 3}-0.05 \mathrm{~T}_{\mathrm{c}}+0.0364 \mathrm{~T}_{\mathrm{c}}^{4 / 3}\right)}
$$

Finally, all units are in the CGS system, except energies which are in keV, currents which are in amps, and powers which are in MW, unless otherwise specified. 
The Maxwell-Boltzmann relation between central-cell electrons and barrier-cell electrons at $z \leq z_{b 0}$ is given by

$$
n^{*}(z)=n_{c} \exp \left(-\frac{\phi_{e}-\phi^{*}(z)}{T_{e c}}\right) \text { for } z \leq z_{b 0} \text {. }
$$

A specific case of Eq. (2-23) is

$$
n_{b}=n_{c} \exp \left(-\frac{\phi_{b}}{T_{e c}}\right)
$$

The Maxwe11-Boltzmann relation between plug electrons and barrier-cell electrons at $z \geq z_{b 0}$ is given by

$$
n^{*}(z)=n_{p} \exp \left(-\frac{\phi_{e}+\phi_{c}-\phi^{*}(z)}{T_{e p}}\right) \quad \text { for } z \geq z_{b 0}
$$

A specific case of Eq. (2-25) is

$$
n_{b}=n_{p} \exp \left(-\frac{\phi_{c}+\phi_{b}}{T_{e p}}\right) \text { for } z \geq z_{b 0} \text {. }
$$

Next, we note that conservation of momentum of passing ions between the central cell and the barrier cell yields the relationship 


$$
n_{\text {pass }}(z) \approx n_{c} \frac{B_{p l a}^{*}(z)}{B_{m b}, p l a}\left\{\frac{T_{c}}{\pi\left[\phi_{e}-\phi^{*}(z)\right]+T_{c}}\right\}^{1 / 2} \text { for } \phi^{*} \leq \phi_{e} \text {. }
$$

A specific case of Eq. $(2-27)$ is

$$
n_{\text {pass }}\left(z_{b 0}\right) \cdot g_{b 0}=n_{b}=n_{c}\left(\frac{g_{b 0}}{R_{b}}\right)\left(\frac{T_{c}}{\pi \phi_{b}+T_{c}}\right)^{1 / 2} .
$$

Next, we apply the Maxwell-Boltzmann relation between passing ions in the central cell and passing ions in the barrier cell to get

$$
n_{p a s s}(z)=n_{c} \frac{B_{p l a}^{*}(z)}{B_{m b, p 1 a}}\left[\frac{\exp \left(-\frac{\phi^{*}(z)-\phi_{e}}{T_{c}}\right)-\exp \left(-\frac{\phi_{c}}{T_{c}}\right)}{1-\exp \left(-\frac{\phi_{c}}{T_{c}}\right)}\right] \text { for } \phi^{*}>\phi_{e},
$$

where the factor $\mathrm{B}^{*}(\mathrm{z}) / \mathrm{B} \mathrm{mb}, \mathrm{pla}$ has been inserted because the passing ions are not isotropic, but rather are only found within the barrier-cell loss cone. We note that Eq. (2-29) matches Eq. (2-27) when $\phi^{*}=\phi_{e}$; indeed, this is how we selected the regimes of validity of Eqs. (2-27) and (2-29). The factors $\exp \left(-\phi_{c} / T_{c}\right)$ are required for the density of passing ions to go to zero at the plug mirror $\left(\phi^{*}=\phi_{e}+\phi_{c}\right)$. An unmodified Maxwell-Boltzmann exponential factor could not do this simply because the passing-ion distribution is not Maxwellian (our modification is tantamount to cutting off the Maxwellian tail at $\left.\frac{1}{2} \mathrm{MV}_{\|}{ }^{2}=\phi_{c}\right)$. This form exhibits the proper behavior as $\phi^{*} \rightarrow_{e}+\phi_{c}$ as we11 as when $\phi^{*} \rightarrow \phi_{e}$.

The perpendicular ion temperature in the barrier is assumed to be $\left(T_{c} / R_{b}\right)$, so $\beta^{*}(z)$ is given by the following equations: 


$$
B^{\star}(z)=\frac{n^{\star}(z)\left(T_{c} / R_{b}\right)+n^{\star}(z) T_{e c}}{\left[B_{v a c}^{\star}(z)\right]^{2} / 2 \mu_{0}} \text { if } z<z_{b 0}
$$

and

$$
B^{*}(z)=\frac{n^{*}(z)\left(T_{c} / R_{b}\right)+\left[n^{*}(z)-n_{b}\right] T_{e p}+n_{b} T_{e c}}{\left[B_{v a c}^{*}(z)\right]^{2} / 2 H_{0}} \text { if } z>z_{b 0} \text {. }
$$

The following is a convenient formulary resulting from algebraic manipulations of the preceding equations. The first of these, Eq. (2-31), is a transcendental equation for $\phi_{b}$, and is obtained by combining Eqs. (2-24) and (2-28) to eliminate $n_{b}$. The second, Eq. $(2-, 2)$, is the equation for $\phi_{c}$, and is obtained by combining Eqs. (2-24) and (2-25) to eliminate $n_{b}$. Equations (2-33) follow from Eqs. (2-17), (2-19), (2-27), and (2-29). Equations (2-34) follow from Eqs. (2-23) and (2-25). Equations (2-35) are a restatement of Eqs. (2-30). Together, Eqs. (2-33), (2-34), and (2-35) constitute three equations for the three unknowns $n^{*}(z), \phi^{*}(z)$, and $\beta^{*}(z)$, assuming $\mathrm{Bvac}_{\mathrm{vac}}^{*}(x)$ is given, at every point in the barrier cell.

$$
\begin{aligned}
& \left(\frac{\phi_{b}}{T_{c}}\right)=\frac{T_{e c}}{T_{c}} \ln \left\{\frac{R_{b}}{g_{b}}\left[1+\pi\left(\frac{\phi_{b}}{T_{c}}\right)\right]^{1 / 2}\right\}, \\
& \phi_{c}=T_{e p} \ln \left(\frac{n_{p}}{n_{c}}\right)+\left(\frac{T_{e P}}{T_{e c}}-1\right) \phi_{b},
\end{aligned}
$$

$n^{*}(z)=g_{b}(z) n_{c} \frac{B_{\text {vac }}^{*}(z)}{B_{m b}, p 1 a}\left[1-B^{*}(z)\right]^{1 / 2}\left\{\frac{T_{c}}{\pi\left[\phi_{e}-\phi^{*}(z)\right]+T_{c}}\right\}^{1 / 2}$ for $\phi^{*} \leq \phi_{e}$, 


$$
\mathrm{n}^{*}(\mathrm{z})=\mathrm{g}_{\mathrm{b}}(\mathrm{z}) \mathrm{n}_{\mathrm{c}} \frac{\mathrm{B}_{\mathrm{vac}}^{*}(z)}{\mathrm{B}_{\mathrm{mb}, \mathrm{pla}}}\left[1-\beta^{*}(z)\right] 1 / 2\left[\frac{\exp \left(-\frac{\phi^{*}(z)-\phi_{\mathrm{e}}}{\mathrm{T}_{\mathrm{c}}}\right)-\exp \left(-\frac{\phi_{c}}{\mathrm{~T}_{\mathrm{c}}}\right)}{1-\exp \left(-\frac{\phi_{c}}{\mathrm{~T}_{\mathrm{c}}}\right)}\right]
$$$$
\phi^{*}(z)=\phi_{e}-T_{e c} \ln \left[n_{c} / n^{*}(z)\right] \quad \text { for } z \leq z_{b 0},
$$$$
\phi^{*}(z)=\phi_{e}+\phi_{c}-T_{e p} \ln \left[n_{p} / n^{*}(z)\right] \text { for } z \geq z_{b 0},
$$

$$
\beta^{*}(z)=\left[n^{*}(z)\left(T_{c} / R_{b}\right)+n^{*}(z) T_{e c}\right] /\left\{\left[B_{v a c}^{*}(z)\right]^{2} / 2 \mu_{0}\right\} \text { for } z \leq z_{b 0} \text {, }
$$

$$
\begin{aligned}
& \beta^{*}(z)=\left\{n^{*}(z)\left(T_{c} / R_{b}\right)+\left[n^{*}(z)-n_{b}\right] T_{e p}+n_{b} T e c\right\} /\left\{\left[B_{v a c}^{*}(z)\right]^{2} / 2 \mu_{0}\right\} \\
& \text { for } z \geq z_{b 0} \text {. }
\end{aligned}
$$

Thus, given a magnetic field profile $\mathrm{B}^{*}{ }_{\text {vac }}(z)$, it is possible to determine the density, potential, and beta profiles as well. The radius of the barrier cell at any point is given by the equation for conservation of magnetic flux:

$$
\pi r_{c}^{2} B_{c c, \operatorname{vac}}\left(1-\beta_{c}\right)^{1 / 2}=\pi\left[r^{*}(z)\right]^{2} B_{v a c}^{*}(z)\left[1-\beta^{*}(z)\right]^{1 / 2} .
$$

Therefore,

$$
r^{*}(z)=r_{c}\left[\frac{{ }_{c c, v a c}}{B_{v a c}^{*}(z)}\left(\frac{1-\beta_{c}}{1-\beta^{*}(z)}\right)^{1 / 2}\right]^{1 / 2} .
$$

A differential volume element in the barrier cell is then 


$$
d V^{*}=\pi\left[r^{*}(z)\right]^{2} d z=\frac{B_{c c, v a c}}{B_{v a c}^{*}(z)}\left(\frac{1-\beta_{c}}{1-\beta^{*}(z)}\right)^{1 / 2} \pi r_{c}^{2} d z \text {. }
$$

Finally, we present the central-cell beta equation and field reduction:

$$
\beta_{c}=\frac{n_{c} T_{c}+n_{c} T_{e c}+P_{\alpha}^{\text {superthermal }}}{\left(B_{c c, v a c}^{2} / 2 \mu_{0}\right)},
$$

and

$$
B_{c c, p l a}=B_{c c, v a c}\left(1-\beta_{c}\right)^{1 / 2} \text {, }
$$

where the superthermal alpha pressure is modeled

$$
\mathrm{p}_{\alpha}^{\text {superthermaI }} \approx 8.3 \times 10^{10} \mathrm{n}_{\mathrm{c}}\langle\sigma \mathrm{v}\rangle{ }_{\mathrm{DT}} \mathrm{T}_{\mathrm{ec}}^{3 / 2} \mathrm{E}_{\alpha} \mathrm{f}_{\alpha \mathrm{e}}
$$

\section{Trapped Ion Pumping in the Thermal Barrier}

The rate at which passing ions trap in the barrier cells per unit barrier-cell volume is given by the following equation, which has been fitted to Fokker-Planck results:

$$
\mathrm{J}_{\text {trap }} \approx \frac{\left[\mathrm{n}_{\mathrm{pass}}(\mathrm{z})\right]^{2}}{5.5 \times 10^{9} \mathrm{~T}_{\mathrm{c}}^{3 / 2}}\left(1+0.55 \frac{\mathrm{B}_{\mathrm{mb}, \mathrm{pla}}}{\mathrm{B}_{\mathrm{pla}}^{\frac{1}{x}}(\mathrm{z})}\right) \text {. }
$$

So the total current at which passing ions trap in the barrier cells is

$$
I_{\text {trap }}=2 \mathrm{e} \int_{\mathrm{z}_{\mathrm{bc}}}^{\mathrm{z} \mathrm{pm}} \frac{\left[\mathrm{n}_{\mathrm{pass}}(\mathrm{z})\right]^{2}}{5.5 \times 10^{9} \mathrm{~T}_{\mathrm{c}}^{3 / 2}}\left(I+0.55 \frac{\mathrm{Bb}_{2} \mathrm{pla}}{\mathrm{B}_{\mathrm{p} 1 \mathrm{a}}^{*}(\mathrm{z})}\right) \mathrm{dV} \mathrm{F}^{*},
$$

where $e$ is the electronic charge, the factor of 2 accounts for both ends of the machine, and the lower limit of integration is taken to be $z_{b c}$ instead of $z_{b m}$ because Eq. $(2-43)$ is not valid (does not approach correct limit) as 
$z \rightarrow z_{b m}$. The cutoff, $z_{b c}$, is defined as that point at which the fraction of velocity space available for trapped particles becomes so small that no greater a value of $g_{b}(z)$ than $g_{b 0}$ is possible. This is defined by

$$
\mathrm{B}_{\mathrm{pla}}^{*}\left(\mathrm{z}_{\mathrm{bc}}\right)=\left(\frac{2 \mathrm{~g}_{\mathrm{b} 0}-1}{\mathrm{~g}_{\mathrm{b} 0}^{2}}\right) \mathrm{B}_{\mathrm{mb}, \mathrm{pla}} \text {. }
$$

Trapped ions that are far enough away from the axis in the barrier cell will have drift surfaces that extend well beyond the plasma boundary. Thus, the barrier-cell plasma will be surrounded by a "halo" of trapped particles. A gas feed into this halo may thus remove some of these trapped particles by either charge exchange or ionization. ${ }^{2-6}$ The fraction of trapped particles that may be so removed by a gas feed is denoted by $f_{D}$. Thus, we define the gas feed current:

$$
\mathrm{I}_{\mathrm{GP}}=\mathrm{f}_{\mathrm{D}} \mathrm{I}_{\text {trap }}
$$

The remaining trapped ions, those are not removed by the gas feed, are pumped by three neutral beams [see Figure 2-1(b)]. On the barrier cell's outboard side (the transition region) there is a low-energy pump beam at $z=z_{\text {LEPB }}$, at the barrier midplane there is a high-energy pump beam at $z=z_{\mathrm{HEPB}}=\mathrm{z}_{\mathrm{bO}}$, and on the barrier cell's inboard side is a medium energy pump beam at $z=z_{M E P B}$. We define

$$
\begin{aligned}
& \phi_{\text {LEPB }} \equiv \phi^{*}\left(z_{\text {LEPB }}\right), \\
& \phi_{\text {MEPB }} \equiv \phi^{*}\left(z_{\text {MEPB }}\right),
\end{aligned}
$$

and

$$
\phi_{\text {HEPB }} \equiv \phi^{*}\left(z_{\text {HEPB }}\right)=\phi_{e}-\phi_{\mathrm{b}} .
$$


These three energetic pump beams must remove the trapping current $\left(1-f_{D}\right) I_{\text {TRAP }}$ that is not removed by the gas pump. They do this by charge exchanging with trapped ions in the barrier ce11, thus producing a passing ion (since the beams are aimed within the loss cone angle of the barrier ce11), and a neutral which leaves the plasma. "The required charge exchange currents are thus

$$
\begin{aligned}
& I_{L E P B}^{c x}=\left(1-f_{D}\right) f_{L E P B}\left[\frac{n^{*}\left(z_{L E P B}\right)}{n^{*}\left(z_{L E P B}\right)-n_{\text {pass }}\left(z_{L E P B}\right)}\right] I_{\text {trap }},(2-46 a) \\
& I_{M E P B}^{C X}=\left(1-f_{D}\right) f_{M E P B}\left[\frac{n^{*}\left(z_{\text {MEPB }}\right)}{n^{*}\left(z_{\text {MEPB }}\right)-n_{\text {pass }}\left(z_{\text {MEPB }}\right)}\right] I_{\text {trap }},(2-46 b)
\end{aligned}
$$

and

$$
I_{H E P B}^{c x}=\left(1-f_{D}\right) f_{H E P B}\left[\frac{n^{*}\left(z_{H E P B}\right)}{n^{*}\left(z_{H E P B}\right)-n_{\text {pass }}\left(z_{H E P B}\right)}\right] I_{\text {trap }}, \quad(2-46 c)
$$

where

$$
\mathrm{f}_{\mathrm{LEPB}}+\mathrm{f}_{\mathrm{MEPB}}+\mathrm{f}_{\mathrm{HEPB}}=1 \text {. }
$$

The Eractions $f_{\text {LEPB }}, f_{M E P B}$, and $f_{\text {HEPB }}$ determine the way in which the total required pumping current is divided among the three energetic beams. The density ratios in square brackets in Eqs. (2-46) are ratios of total ions to trapped ions in the barrier; these are necessary because the beams will

\footnotetext{
*Some of the neutrals from charge exchange of the trapped ions reionize before escape; however, we estimate that the additional neutrals (besides beam neutrals) from charge exchange of passing ions will largely offset the reionization of trapped ions which charge exchange. Thus, we make the approximation here that all trapped ions which charge exchange, escape.
} 
charge exchange with passing ions as well as trapped ions, though the former type of occurrence does not pump.

It must be borne in mind that $I_{\text {LEPB, }}^{c x}$ MEPB, and $I_{\text {HEPB }}^{c x}$ are charge exchange currents; the total beam currents (trapped in plasma) are given by

$I_{L E P B}=\left(1-f_{D}\right) E_{L E P B}\left[\frac{g_{b}\left(z_{L E P B}\right)}{g_{b}\left(z_{L E P B}\right)-1}\right]\left(1+\frac{<\sigma v>_{L E P B}}{<\sigma v>c x}{ }_{L E P B} I_{\text {TRAP }}\right.$,

$\left.I_{M E P B}=\left(1-E_{D}\right) f_{\text {MEPB }}\left[\frac{g_{b}\left(z_{M E P B}\right)}{g_{b}\left(z_{M E P B}\right)-I}\right]\left(1+\frac{<\sigma v>_{M E P B}^{\text {ion }}}{<\sigma v>c x}\right)_{\text {TEPB }}\right)$,

and

$I_{H E P B}=\left(1-f_{D}\right) f_{H E P B}\left[\frac{g_{b}\left(z_{H E P B}\right)}{g_{b}\left(z_{H E P B}\right)-1}\right]\left(1+\frac{<\sigma v>{ }_{H E P B}}{<\sigma v>{ }_{H E P B}^{c x}}\right) I_{\text {TRAP }}$,

where we have used Eq. (2-17). The portions of these currents that are ionized are used to fuel the central cell; this will be important when we write the particle and energy balance equations. For now, we define

$$
\begin{aligned}
& I_{\text {LEPB }}^{\text {ion }}=I_{\text {LEPB }}-I_{\text {LEPB }}^{c x}, \\
& I_{\text {MEPB }}^{\text {ion }}=I_{\text {MEPB }}-I_{M E P B}^{c x},
\end{aligned}
$$

and

$$
I_{\text {HEPB }}^{\text {ion }}=I_{\text {HEPB }}-I_{\text {HEPB }}^{c x}
$$

The injection energies of the three beams are denoted by $V_{L E P B}$, $V_{M E P B}$, and $\mathrm{V}_{\mathrm{HEPB}}$. The requirement that ions produced by ionization and charge ex- 
change of these beams be passing ions (i,e., that they be in the barrier-cell loss cone) sets lower bounds on $V_{M E P B}$ and $v_{\text {HEPB }}$ and an upper bound on $v_{L E P B}$. These bounds may be written

$$
\begin{aligned}
& V_{L E P B}<\frac{\phi_{L E P B}-\phi_{e}}{\left\{\left[\frac{{ }_{m b, P l a}}{B_{p l a}^{*}\left(z_{L E P B}\right)}\right] \sin ^{2} \theta_{L E P B}^{i n j}-1\right\}}, \\
& V_{M E P B} \geq \frac{\phi_{e}-\phi_{M E P B}}{\left\{1-\left[\frac{B_{m b}, P l a}{B_{P L A}^{*}\left(z_{M E P B}\right)}\right] \sin ^{2} \theta_{M{ }_{M P B}}^{i n j}\right\}},
\end{aligned}
$$

and

$$
V_{H E P B} \geq \frac{\phi_{e}-\phi_{H E P B}}{\left\{1-\left[\frac{B_{m b, p l a}}{B_{\mathrm{Pl} l a}^{*}\left(z_{H E P B}\right)}\right] \sin ^{2} \theta_{H E P B}^{i n j}\right\}},
$$

where $\theta_{L E P B}^{i n j}, \theta_{M E P B}^{i n j}$, and $\theta_{\text {HEPB }}^{i n j}$ are the injection angles of the low-, medium-, and high-energy pump beams, respectively. of course, these injection angles must be within the loss cone, so, in the latter two cases, it must be further specified that

$$
\theta_{M E P B}^{i n j} \leq \arcsin \left[\frac{\left.B_{p 1 a^{(z}}^{*}{ }_{m b P B}\right)}{B_{m b}, p l a}\right]^{1 / 2},
$$

and

$$
\theta_{\mathrm{HEPB}}^{i n j} \leq \arcsin \left(\frac{1}{\sqrt{R_{b}}}\right)
$$

Finally, the pumping power necessary is given by

$$
P_{\text {PUMP }}=\left(I_{\text {LEPB }} V_{\text {LEPB }}+I_{\text {MEPB }} V_{\text {MEPB }}+I_{\text {HEPB }} V_{\text {HEPB }}\right) / 1000.0 \text {, }
$$


where the factor of 1000.0 yields an answer in MW, since the currents are in amperes, and the energies are in keV.

\section{Plug Mode1}

The plug mode 1 used is essentia11y identical to that developed by Logan and Rensink in 1978, and used in previous TMR studies at Livermore. The model is an analytic fit to Fokker-Planck studies of plug mirror confinement.

Let the potential drop seen by the plug ions be denoted by $\phi_{\mathrm{p}}$. Thus

$$
\phi_{p}=b_{e}+\phi_{c}
$$

The plug vacuum mirror ratio and plug plasma mirror ratio are given by

$$
\mathrm{R}_{\mathrm{p}, \mathrm{vac}} \equiv \mathrm{B}_{\mathrm{mp}, \mathrm{vac}} / \mathrm{B}_{\mathrm{O}_{\mathrm{p}}, \mathrm{vac}}
$$

and

$$
\mathrm{R}_{\mathrm{P}} \equiv \mathrm{B}_{\mathrm{mp}, \mathrm{pla}} / \mathrm{B}_{0 \mathrm{p}, \mathrm{pla}}
$$

respectively. There is no field reduction at the plug mirror, and the "longthin approximation" is used at the plug midplane:

$$
B_{m p, p 1 a}=B_{m p, v a c} \text {, }
$$

and

$$
\mathrm{B}_{0 \mathrm{p}, \mathrm{p} 1 \mathrm{a}}=\mathrm{B}_{0 \mathrm{p}, \mathrm{vac}}\left(1-\beta_{\mathrm{p}}\right)^{1 / 2}
$$

First we define

$$
E_{c} \equiv \frac{\phi_{p}}{R_{p} \sin ^{2} \theta_{i n j}-1},
$$

and 


$$
R_{p, \text { eff }} \equiv \frac{R_{p} \sin ^{2} \theta_{\text {inj }}}{1+\left(\phi_{p} / E_{p, i n j}\right)}
$$

where $\theta_{i n j}$ is the injection angle of the plug neutral beam, and $E_{p, i n j}$ is the injection energy of the plug neutral beams, respectively. Then we calculate

$$
\ln \Lambda_{\mathrm{ei}} \approx \ln \left(5.7 \times 10^{13} \mathrm{~T}_{\mathrm{ep}} / \sqrt{\mathrm{n}_{\mathrm{p}}}\right)
$$

and

$$
\ln \Lambda_{i \mathrm{i}} \approx \ln \left[1.4 \times 10^{15}\left(\mathrm{~T}_{\mathrm{ep}} \mathrm{E}_{\mathrm{p}} / \mathrm{n}_{\mathrm{p}}\right)^{1 / 2}\right],
$$

where $E_{p}$ is the plug ion energy (for which one must provide an initial guess to begin this calcuation).

Next, we solve the following two equations numerically for $\left(\bar{E}_{L} / E_{p}\right.$, inj $)$ and $\left(\tau_{i i} / \tau_{\mathrm{drag}}\right)$ :

$$
\left(\frac{\bar{E}_{L}}{E_{p, i n j}}\right)=\frac{1+\left(E_{c} / E_{p, i n j}\right)\left(\tau_{i i} / \tau_{d r a g}\right)}{1+\left(\tau_{i i} / \tau_{d r a g}\right)},
$$

and

$$
\left(\frac{\tau_{i i}}{\tau_{d r a g}}\right)=\frac{0.156}{\sqrt{A}}\left(\frac{E_{p, i n j}}{T_{\text {e.p }}}\right)^{3 / 2} \frac{\log _{10}\left(R_{p, e f f}\right)}{\ln \left[1 /\left(\bar{E}_{L} / E_{p, i n j}\right)\right]}\left(\frac{l_{n} \Lambda_{e i}}{\ln \Lambda_{i i}}\right),
$$

where $A_{p}$ is the plug ion mass in AMU. Then we calculate

$$
\begin{aligned}
& \left(\mathrm{n} \tau_{\mathrm{drag}}=1.0 \times 10^{13} \mathrm{~A}_{\mathrm{p}} \mathrm{T}_{\mathrm{ep}}^{3 / 2} / 1 \mathrm{n} \Lambda_{\mathrm{ei}},\right. \\
& \left(n_{\tau}\right)_{p}^{i i} \equiv 2.76 \times 10^{12} \sqrt{A_{p}} E_{p, i n j}^{3 / 2} \log _{10}\left(R_{p, e f f}\right) / \ln \Lambda_{i i}, \\
& (n \tau)_{p}^{e i} \equiv 1.0 \times 10^{13} A_{p} T_{e p}^{3 / 2} \ln \left[1 /\left(\bar{E}_{L} / E_{i n j}\right)\right] / \ln \Lambda_{e i},
\end{aligned}
$$


and

$$
(n \tau)_{p}=\left\lfloor\frac{1}{(n \tau)_{p}^{i j}}+\frac{1}{(n \tau)_{p}^{e i}}\right\rfloor^{-1}
$$

To complete the iterative process, we use the ion energy balance of the plug (neglecting charge exchange) to solve for

$$
E_{P}=\frac{3}{2} T_{e p}+\frac{(n \tau)_{d r a g}}{(n \tau)_{p}}\left(E_{p, i n j}-\bar{E}_{L}\right)
$$

This new value of $\mathrm{E}_{\mathrm{p}}$ is then inserted back into $\mathrm{Eq}$. (2-58b), and the entire process is repeated until it converges onto a self-consistent set of parameters. At very high values of $\mathrm{T}_{\mathrm{ep}}$, such as are encountered in tandem mirrors with barrier cells, it has been found that the Logan-Rensink plug model can yield anomalously high values of $\mathrm{E}_{\mathrm{p}}$. Consequently, in this study, if we obtain $E_{p}>2.5 E_{p, i n j}$, we set $E_{p}=2.5 E_{p, i n j}$ before beginning the next iteration. In this way, we ensure $E_{p} \leq 2.5 E_{p}, i_{n j}$.

The particle balance equation for the plug ions may be written

$$
I_{p, i n j}=e \frac{n_{p}^{2}}{(n \tau)_{p}} \cdot\left(\frac{4 \pi}{3} r_{p}^{3}\right)
$$

where $I_{p, i n j}$ is the neutral beam current into one plug, e is the electronic charge, and $r_{p}$ is the plug radiug given by conservation of magnetic flux:

$$
r_{p}=r_{c}\left[\frac{B_{c c, v a c}}{B_{O_{p}, v a c}}\left(\frac{1-\beta_{c}}{1-\beta_{p}}\right)^{1 / 2}\right]^{1 / 2} \text {. }
$$

The total neutral-beam power into both plugs is then

$$
P_{N B}=\left(2 I_{p, i n j} E_{p, i n j}\right) / 1000.0,
$$


where the factor of 2 accounts for both ends of the machine, and the factor of 1000.0 yields an answer in $M W$, since $I_{p, \text { inj }}$ is in amperes and $E_{p \text {, inj }}$ is in keV. Finally, we present the plug beta equation:

$$
E_{p}=\frac{n_{p}\left(0.9 E_{p}\right)+n_{p} T_{e p}}{\left(B_{0 p}^{2}, v_{a c} / 2 \mu_{0}\right)},
$$

where the factor of 0.9 accounts for the fact that most of the plug ion energy is perpendicular (the factor would be $2 / 3$ for a perfectly isotropic plasma).

\section{The Particle and Energy Balance Equations}

The particle balance of the central-cell ions is written ${ }^{*}$

$\frac{n_{c}^{2} v_{c}}{(n \tau)_{c}}+\frac{1}{2} n_{c}^{2}\langle\sigma v\rangle_{D T} v_{c}+I_{G P}=I_{L E P B}^{i o n}+I_{M E P B}^{i o n}+I_{\text {HEPB }}^{\text {ion }}+I_{A U X}^{i o n}$,

where

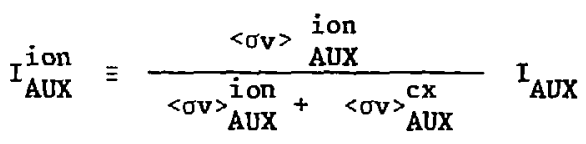

and

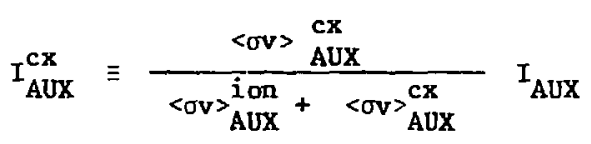

comprise an auxiliary fuel source located at the central-cell potential,

$$
(n \tau)_{c} \approx 5.547 \times 10^{11} \frac{g\left(R_{c i} / 2\right)}{\ln \Lambda_{i i}} T_{c}^{3 / 2}\left(\frac{\phi_{c}}{T_{c}}\right) \exp \left(\frac{\phi_{c}}{T_{c}}\right)
$$

*Throughout this section, we neglect radial loss compared to end loss for DT fuel ions as well as electrons. No thermal alpha population is assumed to be present. 
is the Pastukhov ( $\mathrm{n} \tau)$,

$$
\begin{aligned}
& \mathrm{R}_{\mathrm{ci}} \equiv \mathrm{B}_{0 \mathrm{p}, \mathrm{pla}} / \mathrm{B}_{\mathrm{cc}, \mathrm{pla}}, \\
& \ln \Lambda_{\mathrm{ii}}=\ln \left[3.08 \times 10^{14}\left(\frac{\mathrm{T}_{\mathrm{c}}^{3 / 2}}{\mathrm{n}_{\mathrm{c}} 1 / 2}\right)\right],
\end{aligned}
$$

and

$$
g(x)=\frac{\sqrt{\pi}}{2}\left(1+\frac{1}{x}\right)^{1 / 2} \ln \left\{\left[\left(1+\frac{1}{x}\right)^{1 / 2}+1\right] /\left[\left(1+\frac{1}{x}\right)^{1 / 2}-1\right]\right\} .
$$

The first term on the left-hand side of Eq. (2-70) is the Pastukhov end loss of central-cel1 ions, and the second term is the ion burnup rate in fusion reactions. The third term on the left-hand side of Eq. (2-70) reflects the following fact: The current of passing ions that becomes trapped is, in steady-state, exactly equal to the current of trapped ions that is pumped back into passing space minus the current of trapped ions that is pumped by the gas feed. Any trapped ion that either ionizes or charge exchanges with the gas feed produces an ion that, being born on a flux surface so far outside of the plasma, is essentially lost from the plasma. Thus, the entire gas-feed current is a drain on the central-cell ion population.

The first three terms on the right-hand side of Eq. (2-70) are the fueling due to the pump beams, and the last term on the right-hand side is the ionization current of an auxiliary fueling source. It may be preferable to do all fueling via the pump beams, in which case this term would be equal to zero.

The central-ce11 ion energy balance may be written (note: $E_{c, i n j}$ may be zero if, for example, pellets are used for auxiliary fueling): 


$$
\begin{aligned}
& \frac{1}{4} n_{r}^{2}\langle\sigma V\rangle_{D T}\left(E_{\alpha}\left(1+\frac{E_{R}}{E_{F U S}}\right) E_{\alpha i}-E_{R}\right] v_{c}-\frac{n_{c}^{2} v_{c}}{(n \tau)_{c}}\left(t_{c}+T_{c}\right) \\
& +\frac{n_{c}^{2} V_{c}}{(n \tau)_{e i}} \cdot \frac{3}{2}\left(T_{e c}-T_{c}\right)+\left[I_{A U X}^{i o n} E_{c, i n j}+I_{A U X}^{c x}\left(E_{c, i n j}-\frac{3}{2} T_{c}\right)\right. \\
& +I_{\text {LEPB }}^{\text {ion }}\left(V_{L E P B}+\phi_{L E P B}-\phi_{e}\right)+I_{L E P B}^{c x}\left(V_{L E P B}+\phi_{L E P B}-\phi_{e}-\frac{3}{2} T_{c}\right) \\
& +I_{M E P B}^{i \text { on }}\left(v_{M E P B}+\phi_{M E P B}-\phi_{e}\right)+I_{M E P B}^{c x}\left(v_{M E P B}+\phi_{M E P B}-\phi_{e}-\frac{3}{2} T_{c}\right) \\
& +\mathrm{I}_{\mathrm{HEPB}}^{\text {ion }}\left(\mathrm{V}_{\mathrm{HEPB}}+\phi_{\mathrm{HEPB}}-\phi_{\mathrm{e}}\right)+\mathrm{I}_{\mathrm{HEPB}}^{\mathrm{cx}}\left(\mathrm{V}_{\mathrm{HEPB}}+\phi_{\mathrm{HEPB}}-\phi_{\mathrm{e}}-\frac{3}{2} \mathrm{~T}_{\mathrm{c}}\right) \\
& \left.-I_{G P}\left(\frac{3}{2} T_{c}\right)\right] / e=0
\end{aligned}
$$

where

$$
\begin{aligned}
& \mathrm{E}_{\mathrm{R}} \approx 45.0+\frac{3}{2} \mathrm{~T}_{\mathrm{c}}, \\
& (\mathrm{n} \tau)_{\mathrm{ei}} \approx \frac{2.50 \times 10^{13} \mathrm{~T}_{\mathrm{ec}}^{3 / 2}}{1 ; \Lambda_{\mathrm{ei}}}
\end{aligned}
$$

and

$$
\operatorname{In} \Lambda_{\mathrm{ei}} \approx \operatorname{In}\left[2.65 \times 10^{13} \mathrm{~T}_{\mathrm{ec}} / \sqrt{\mathrm{n}_{\mathrm{c}}}\right]
$$

The first term of Eq. $(2-76)$ is the alpha heating of ions; $E_{R}$ is the reactant energy, which is assumed to be distributed between the fusion neutron and fusion a1pha in the same proportion as is the fusion energy. The second term is the Pastukhov energy loss out the ends. The third term is the energy transfer between ions and electrons. The fourth, sixth, eighth, and tunth terms are the energy gains due to ionization of the auxiliary fuel beam, LEPB, 
MEPB, and HEPB, respectively. The fifth, seventh, ninth, and eleventh terms are the charge-exchange energy transfers between the auxiliary fuel beam, LEPB, MEPB, and HEPB, respectively. Finally, the twelfth term is the energy loss due to either ionization or charge exchange with the gas feed.

The central-cell (passing) electron particle balance, which determines $\phi_{\mathrm{e}}$, may be written as follows:

$$
\left(I_{L E P B}^{\text {ion }}+I_{M E P B}^{\text {ion }}+I_{\text {HEPB }}^{\text {ion }}+I_{A U X}^{\text {ion }}+I_{s}\right) / e+\frac{n_{p}^{2}\left(2 V_{p}\right)}{(n \tau)_{P}}=\frac{n_{c}^{2} v_{c}}{(n \tau)_{e c}} \text {, }
$$

where

$$
(\mathrm{n} \tau)_{\text {ec }} \approx 8.19 \times 10^{9} \frac{g\left(\mathrm{R}_{\mathrm{ce}} / 2\right)}{\ln \Lambda_{\mathrm{ee}}} \mathrm{T}_{\mathrm{ec}}^{3 / 2}\left(\frac{{ }_{\mathrm{e}}}{\mathrm{T}_{\mathrm{ec}}}\right) \exp \left(\frac{\mathrm{e}}{\mathrm{T}_{\mathrm{ec}}}\right),
$$

and

$$
\ln \Lambda_{\mathrm{ee}} \approx \ln \left(2.83 \times 10^{13} \mathrm{~T}_{\mathrm{ec}} / \sqrt{\mathrm{\pi}_{\mathrm{c}}}\right)
$$

The first four terms on the left-hand side of Eq. (2-80) are the electrons produced by ionization of the LEPB, MEPB, HEPB, and auxiliary fuel beam, respectively. The fifth term is an auxiliar. current, $I_{s}$, of cold electrons that one may wish to inject into the central $=11$. The sixth term reflects the fact that every time a plug ion is lost out the end of the machine, a plug electron must become a passing electron in order to maintain quasi-neutrality in the plugs. The term on the right-hand side of Eq. (2-80) is the Pastukhov end loss of electrons.

The central-cell (passing) electron energy balance may be written as follows (note: potential energies in this equation are referenced to the centra1-cel1 potentia1, $\left.\phi_{\mathrm{e}}\right)$ : 


$$
\begin{aligned}
& \left.\frac{1}{4} n_{c}^{2}<\sigma v\right\rangle_{D T}\left[E_{\alpha}\left(1+\frac{E_{R}}{E_{F U S}}\right) E_{\alpha e}\right] v_{c}+\frac{1}{e} I_{s} \phi_{e} \\
& -\frac{n_{c}^{2} v_{c}}{(n \tau)_{e c}}\left(\phi_{e}+T_{e c}\right)+\frac{n_{p}^{2}\left(2 V_{p}\right)}{(n \tau)_{p}}\left(\phi_{b}+T_{e p}\right)-\frac{n_{c}^{2} v_{c}}{(n \tau)_{e i}} \cdot \frac{3}{2}\left(T_{e c}-T_{c}\right) \\
& +P_{c P}^{*}+P_{c P}=\frac{-1}{e} \int I_{L E P B}^{\text {ion }}\left(\phi_{b}+\bar{E}_{e ~ L E P B}\right) \\
& +I_{M E P B}^{\text {ion }}\left(\phi_{e}-\phi_{\text {MEPB }}\right) \\
& \left.+I_{\mathrm{HEPB}}^{\text {ion }}\left(\phi_{b}\right)\right] \text {, }
\end{aligned}
$$

where

$$
\begin{aligned}
& \overline{\mathrm{E}}_{\mathrm{e} L E P B} \approx \phi_{\mathrm{b}}+\phi_{\mathrm{LEPB}}-\phi_{\mathrm{e}} \text {, } \\
& P_{c p} \approx G_{e p} \frac{n_{p}^{2}}{(n \tau)_{p}^{e e}}\left(T_{e p}-T_{e c}\right) \exp \left(-\frac{\phi_{c}+\phi_{b}}{T_{e p}}\right) \quad\left(2 v_{p}\right), \\
& P_{c p}^{*} \approx \int_{z_{b O}}^{z} G_{e t} \frac{n_{p}^{2}}{(n \tau)_{t}^{e e}}\left(T_{e p}-T_{e c}\right) \exp \left(-\frac{\phi_{e}+\phi_{c}-\phi^{*}(z)}{T_{e p}}\right) 2 \cdot d V^{*}, \\
& G_{\mathrm{ep}} \approx G_{\mathrm{et}} \approx 1, \\
& \left(\mathbf{n}_{\tau}\right)_{\mathrm{p}}^{\mathrm{ee}} \approx 8.19 \times 10^{9} \frac{\mathrm{T}_{\mathrm{ep}}^{3 / 2}}{\ln \Lambda_{\mathrm{p}}^{\mathrm{ee}}}
\end{aligned}
$$




$$
\begin{aligned}
& (n \tau)_{t}^{e e}=8.19 \times 10^{9} \frac{\mathrm{T}_{\mathrm{ep}}^{3 / 2}}{\ln \Lambda_{t}^{\mathrm{ee}}}, \\
& \ln \Lambda_{\mathrm{p}}^{\mathrm{ee}}=\ln \left(2.83 \times 10^{13} \mathrm{~T}_{\mathrm{ep}} / \sqrt{\pi_{\mathrm{p}}}\right),
\end{aligned}
$$

and

$$
\ln \Lambda_{t}^{e e}=\ln \left\{2.83 \times 10^{13} T_{\text {ep }} /\left[n^{*}(z)\right]^{1 / 2}\right\}
$$

The first term on the left-hand side of Eq. (2-83) is the alpha heating of the passing electrons. The second term on the left is the gain due to the influx of cold auxiliary electrons [see fourth and Fifth terms of Eq. (2-80)]. The third term on the left is the Pastukhov energy loss. The fourth term on the left is the energy gain each time a trapped electron becomes a passing electron. The fifth term on the left is the energy transfer between central-cell ions and electrons. The sixth and seventh terms on the left model the thermal energy transfer between the passing electrons and the trapped electrons in the transition region and plug, respectively.

The terms on the right-hand side of Eq. (2-83) are contributions to passing-electron energy due to the arrival of electrons in the LEPB, MEPB, and HEPB, respectively.

The final energy balance we present to complete the system of equations describing the barrier cell tandem mirror is the trapped electron energy balance: 


$$
\begin{aligned}
& P_{E C R H}+\frac{n_{p}^{2}\left(2 v_{p}\right)}{(n \tau)_{d r a g}}\left(E_{p}-\frac{3}{2} T_{e p}\right)-\frac{n_{p}^{2}\left(2 v_{p}\right)}{(n \tau)_{p}}\left(\phi_{c}+\phi_{b}+T_{e p}\right) \\
& =P_{c P}^{*}+P_{C P}+P_{\text {synchrotron }}+\frac{1}{e} I_{\text {LEPB }}^{\text {ion }} \mid\left(\phi_{L E P B}+\phi_{b}-\phi_{e}\right)+\bar{E}_{e} \text { LEPB } \mid \cdot(2-90)
\end{aligned}
$$

The first term on the left-hand side of Eq. (2-90) is the ECRH power input, and the second term is the heating due to plug ion drag. The third term is the energy loss due to cooling by incoming neutral-beam electrons. The first two terms on the right-hand side are the energy losses to the passing electrons in the transition region and plug, respectively. The third term on the right is the loss of energy due to synchrotron radiation in the plugs and transition regions. The final term is the cooling effect of electrons that arrive with the LEPB.

Finally, we take the fusion power to be given by

$$
\left.P_{\text {fusion }}=\frac{1}{4} n_{c}^{2}<\sigma V\right\rangle_{D T} E_{F U S}\left(\pi r_{c}^{2} L_{c}\right),
$$

and the neutron wall loading to be given by

$$
\Gamma=\left(E_{n} / E_{\text {FUS }}\right) P_{\text {fusion }} /\left(2 \pi r_{F W} L_{c}\right)
$$

TMR SCALING WITH THERMAL BARRIERS

At presert, there are many pieces of the physics model described in the preceeding section, such as the plug-to-center-cell electron transfer power $P_{c p}$, the relationship between $\phi_{c}+\phi_{b}, T_{e p}, T_{e c}$ and $n_{p}, n_{b}$, the precise barrier ion trapping rate $J_{\text {trap' }}$ and the center-cell MHD beta limit, which are only crudely estimated and which are the subject of ongoing theoretical investigation. Until uncertainties associated with these parts of the physics model are resolved by further work, a detailed point design for a TMR with 
thermal barriers is unwarranted. However, at this time we have identified the essential physics and basic scaling laws for the thermal barrier concept, and so we can project trends in the various parameters that should lead us to the TMR goals set forth in the first section of this report: $Q \geq 10$, $\mathrm{p}_{\text {net }} \leq 500 \mathrm{MWe}, \mathrm{L}_{\mathrm{c}} \leqslant 50 \mathrm{~m}\left(\Gamma \geq 2 \mathrm{MH} / \mathrm{m}^{2}\right)$, and $\mathrm{B}_{\text {cond }} \leqslant 12 \mathrm{~T}$.

The most important and, at present, least known parameter is the centercell beta limit $B_{c}$, which is determined by MHD stability considerations, ballooning in particular. The ballooning-mode beta limit for $\sigma_{c}$ will depend on plasma pressure profiles in the barrier (pumping), radial electric fields (rotation), and detailed magnet design (field-line lengths and curvatures). Radial electric fields may or may not exist, depending on whether radial electron transport rates compete with end loss. The first iteration magnet design described in Chapter 3, developed only with interchange as an MHD stability constraint, has been subsequently evaluated for ballooning by an MHD $\operatorname{code}^{2-8}$ recently modified to treat geometries appropriate for tandem mirrors with thermal barriers. The resulting low beta limit ( $\beta_{c} \leq 10 \%$ ) for ballooning stability in the present thermal barrier TMR design, as compared to stable betas $B_{c} \approx 50 \%$ found in previous tandem mirror design appears to be due in part to the rather long length of the present thermal barrier regions $\left(L_{b} \geq 14 \mathrm{~m}\right)$, which reduçes field line-bending energy. Ongoing magnet design efforts seek to improve the center-cell beta by reducing the length of the thermal barrier.

Since the center-cell beta and the thermal barrier length $L_{b}$ are important unresolved parameters, we characterize the TMR $Q$ scaling in terms of these parameters in an approximate way as follows: assuming a sufficiently small barrier density $n_{b} \ll n_{c}$ makes the pump power dominate, we use Eq. (2-14) for Q. Integrating the ion-trapping current density Eq. (2-41) over the axial field profile of the barrier cell in the present magnet set, 
and assuming that half of the pumping is achieved by charge exchange with appropriate neutral beams injected into the barrier as described in Chapter 5 (the other half of the trapped ion current is assumed to be lost radially by non-omigeneous drift surfaces ${ }^{2-6}$ we can estimate a pump-power (trapped) scaling as

$$
P_{\text {pump }} 2 V_{b} \approx k_{b} \frac{\pi_{c}^{2}}{T_{i c}^{3 / 2}}\left[\frac{\pi r_{c}^{2} B_{c}\left(1-\beta_{c}\right)^{1 / 2}}{B_{a b}}\right] L_{b} \phi_{b}(M W),
$$

where the proportionality constant $k_{b} \approx 3.6 \times 10^{-41} \mathrm{~T}_{\mathrm{ic}}{ }^{3 / 2}$ is a weak function of the axial field profile shape in the barrier, with the indicated temperature dependence valid in the range $20<\mathrm{T}_{\mathrm{ic}}<40 \mathrm{keV}$. Here $\mathrm{n}_{\mathrm{c}}$ is in $\mathrm{m}^{-3}$, $r_{c}$ and $L_{b}$ in metres, $T_{i c}$ and $\phi_{b}$ in keV. With this expression for $P_{\text {pump }} 2 V_{b}$, and approximating $\phi_{b} \approx 3 \mathrm{~T}_{\mathrm{ic}}\left(\phi_{\mathrm{b}} / \mathrm{T}_{\mathrm{ic}}\right.$ varies only logarithmically), Eq. (2-14) for $Q$ can be written as

$$
\begin{aligned}
& Q \approx \frac{\frac{1}{4} \mathrm{n}_{c}^{2}\langle\sigma \mathrm{V}\rangle_{\mathrm{DT}}(17.6)\left(1.6 \times 10^{-19}\right) \pi r_{c}^{2} \mathrm{~L}_{c}}{k_{b} \frac{n_{c}^{2}}{T_{i c}^{3 / 2}}\left[\frac{r_{c}^{2} B_{c}\left(1-\beta_{c}\right)^{1 / 2}}{B_{m b}}\right] L_{b} \phi_{b}} \\
& Q \approx 6.5 \times 10^{21}\left(\frac{\left\langle\sigma v{ }_{D T}\right.}{T_{i c}}\right)\left[\frac{B_{m b}}{B_{c}\left(1-\beta_{c}\right)^{1 / 2}}\right]\left(\frac{L_{c}}{L_{b}}\right) .
\end{aligned}
$$

We note that $\left\langle\sigma v{ }_{\mathrm{DT}} / \mathrm{T}_{\text {ic }}\right.$ has a broad maximum around $\mathrm{T}_{i \mathrm{c}} \approx 30 \mathrm{keV}$. We can take the maximum $B_{m b} \approx 12 T$, assuming the solenoidal barrier coil can have an efficiency approaching $100 \%$. An economic neutron wall loading $\Gamma \approx 3 \mathrm{MW} / \mathrm{m}^{2}$ is achieved for a center-cell plasma radius $r_{c} \approx 1$ m and first-wall radius $r_{w} \approx 1.5 \mathrm{~m}$, accomnodatable in the present magnet set, at a center-cell density $n_{c} \approx 1.4 \times 10^{20} \mathrm{~m}^{-3}$. For this density, the required center-cell field scales as $B_{c} \approx 2.1 / \sqrt{B_{c}}$ (tesla), assuming $T_{e c}=T_{i c}$, including alpha pressure. 
With these assumptions, and taking $\mathrm{P}_{\text {fusion }} \mathrm{v}_{c} /\left[\left(\mathrm{P}_{\mathrm{ECRH}}+\mathrm{P}_{\mathrm{NB}}\right) 2 \mathrm{v}_{\mathrm{p}}\right] \approx \mathrm{L}_{\mathrm{c}}$ (in $\mathrm{m}$ ) to take into account the plug power, the overall $Q$ reduces to roughly

$$
Q \approx\left\{\left[0.8\left(\frac{B_{c}}{1-B_{c}}\right)^{1 / 2} \frac{L_{c}}{L_{b}}\right]^{-1}+L_{c}^{-1}\right\}^{-1} .
$$

Figure 2-2 shows a $p$ lot of $Q$ versus $L_{b}$ for several values of $\beta_{c}$ and for $a$ center-cell length $L_{c}=50 \mathrm{~m}$, which gives a reactor power $P_{\text {net }} \approx 500 \mathrm{MW}(\mathrm{e})$. Longer $L_{c}$ give proportionally more $Q$ and $P_{\text {net }}$. We see from Fig. 2-2 that we need $\beta_{c}$ in the range of 60 to $70 \%$ at barrier lengths of 5 to 6 metres in order to meet our reactor goals of $Q=10$ at $\mathrm{L}_{c}=50 \mathrm{~m}[500 \mathrm{MW}(\mathrm{e})]$. To achieve $Q=10$ at lower $B_{c}$ would require longer center-cell lengths and corresponding$1 y$ more power.

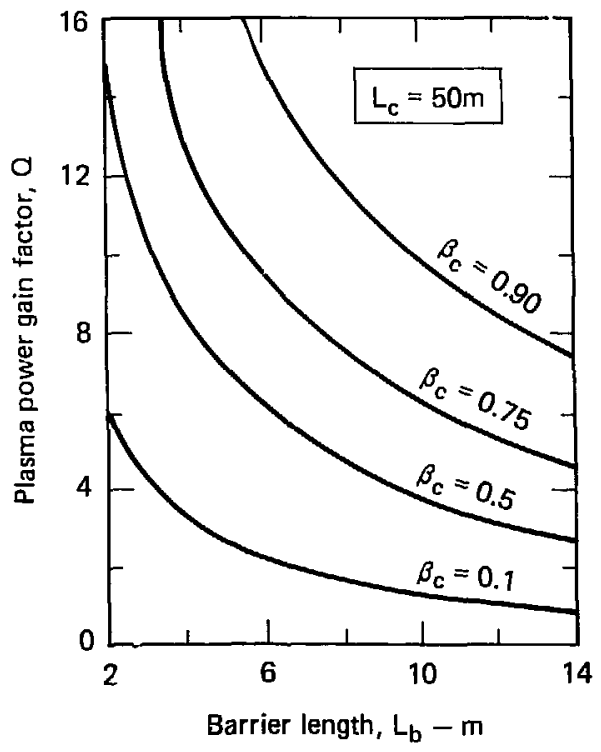

Fig. 2-2. Plasma gain factor, Q, with thermal barriers as a function of barrier length for center cell length $\mathrm{L}_{\mathrm{c}}=50 \mathrm{~m}$. 
If our present design approaches for adding thermal barriers to a TMR (as in Fig. 2-1) do not ultimately yield a satisfactory center-cell MHD beta limit for a small reactor, we can explore other magnet design options to improve the center-cell beta. One promising approach we are presently examining capitalizes on the fact that the shorter transition regions between center cel1 and yin-yang plugs in standard tandem mirrors without thermal barriers already have ballooning beta limits $\beta_{c} \approx 0.5$. The idea is to add thermal barriers between the yin-yang plugs and the A-cells of a previous IMR design, ${ }^{2-4}$ as schematically shown in Fig. 2-3. The yin-yang plugs, operated at lower density, magnetic field, and beam power, would serve primarily as MHD anchors close to the center cel1, where beta can then be high. The Iower density A-cells, which previously served only to reduce the potential drop from the yin-yang plugs, would now be ECRH-heated to a potential greater than the yin-yang plugs, so that the A-cells have the primary function of electrostatic confinement of the center-cell ions. The required ECRH power to raise the A-cell potential would be small because of the thermal barriers added to the gaps between the yin-yang plugs and the A-cel1s. Since the functions of MKD anchor and electrostatic plug are now separated, the A-cell at low betas need not also be minimum-B, allowing flexibility in its magnet design. Pursuit of this promising candidate for a TMR with thermal barriers may result in improved performance through its greater flexibility as well as higher center-cel1 beta. 

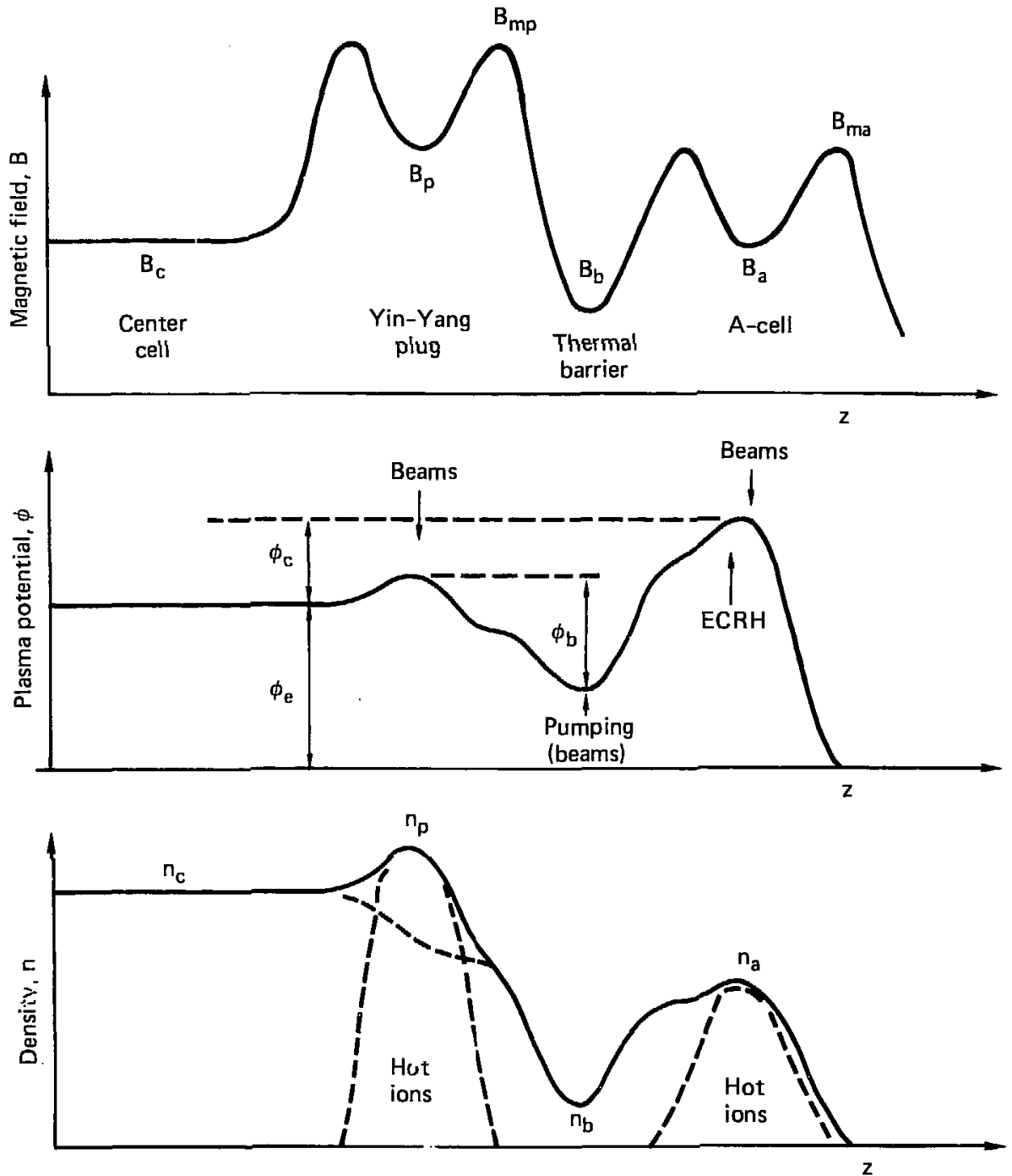

Fig. 2-3. Schematic of magnetic field (B), plasma potential ( $\phi$ ), and density $(n)$ as functions of $z$ for tandem mirror reactor with thermal barriers and A-cells. 
2-1. R. W. Moir, W. L. Barr, G. A. Carlson, W. L. Dexter, J. N. Doggett, J. H. Fink, G. W. Hamilton, J. D. Lee, B. G. Logan, W. S. Neef, Jr., M. A. Peterson, and M. E. Rensink, Preliminary Design Study of the Tandem Mirror Reactor, Lawrence Livermore Laboratory Rept. UCRL-52302 (1977).

2-2. B. G. Logan, W. I. Barr, D. J. Bender, G. A. Carlson, W. L. Dexter, J. N. Doggett, R. S. Devoto, J. F. Fink, T. K. Fowler, G. W. Hamilton, D. Lappa, J. D. Lee, W. D. Neef, Jr., A. A. Mirin, R. W. Moir, M. A. Peterson, and M. E. Rensink, in Proc. 7th Intern. Conf. on Plasma Physics and Cont. Fusion Research, Innsbruck, Austria, 1978 (IAEA, in press); also Lawrence Livermore Laboratory, Rept. UCRL-80644 (1978).

2-3. J. F. Clauser, R. K. Goodman, and G. M. Melin, "Measurements of Axial Electron Temperature Profiles in 2XIIB," Bull. Am. Phys. Soc. 23, p. 775 (1978).

2-4. G. A. Carlson, B. M. Boghosian, J. H. Fink, J. O. Myall, and W. S. Neef, Jr., Parametric Studies of Tandem Mirror Reactors, Lawrence Livermore Laboratory, Rept. UCID-18158 (1979).

2-5. D. E. Baldwin, B. G. Logan, and T. K. Fowler, An Improved Tandem Mirror Fusion Reactor, Lawrence Livermore Laboratory, Rept. UCID-18156 (1979).

2-6. J. Kesner, Passive Generation of Ambipolar Potential Barriers in a Tandem Mirror, University of Wisconsin Report UWFDM-303 (May 1979).

2-7 D. L. Galbraith and T. Kammash, The Dynamic Behavior of a Mirror Fusion Reactor, EPRI. Rept. ER-521 (1978).

2-8. T. B. Kaiser, "Numerica1 Investigation of the Ballooning Stability of TMX at Finite $\beta, "$ Bull. Am. Phys. Soc. 23, 754 (1978). 
CHAPTER 3. MAGNETS

J. 0. Myall

Introduction . . . . . . . . . . . . 67

Central Cell . . . . . . . . . . . . . 69

Barrier Coil . . . . . . . . . . . . . 69

Transition Coil ................ . 72

Plug Yin-lang Coils . . . . . . . . . . . . . 74

Circularizing Coil .............. . 75

Alternative Reactor Design with

Solenoidal Plug Coils . . . . . . . . . . . . 78

References .................. . 80 
The arrangement of magnets that we studied for the tandem mirror reactor is shown in Figure $3-1$. The components are:

- Central ce11 solenoid (actually a series of short solenoidal coils with gaps for blanket coolant pipes)

- Barrier solenoidal coil

- Inner transition "cee" coil

- Plug yin-yang coils

- Outer circularizing "cee" coil.

The total system is symmetric about the center except for a $90^{\circ}$ rotation about the axis.

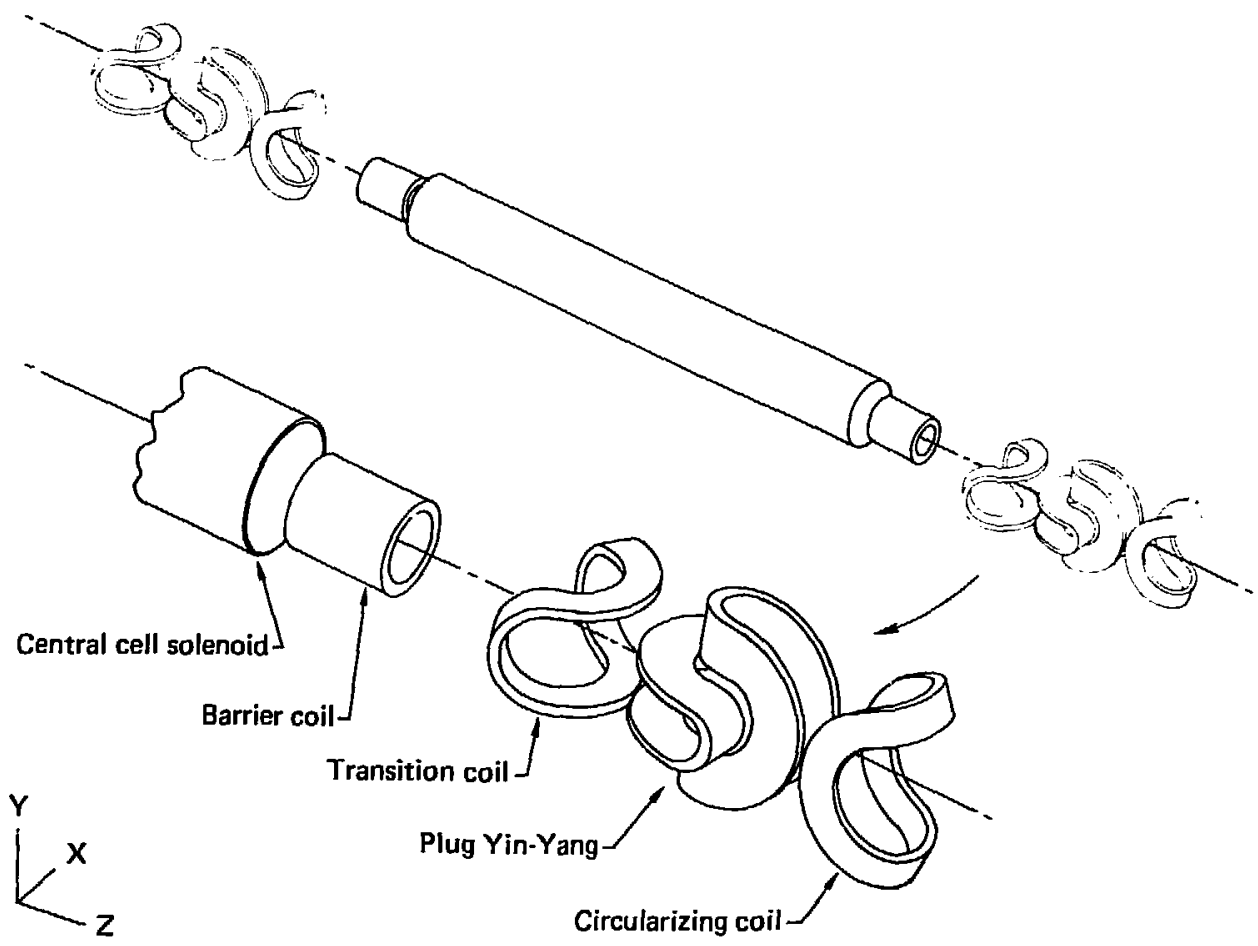

Fig. 3-1. Arrangement of magnets. 
The magnets have been designed by means of the EFFI computer code ${ }^{3-1}$. They give the magnetic field values indicated in Fig. 3-2, which shows the variation in field magnitude on the machine axis. The shape of the curve is characteristic of tandem mirrors incorporating thermal barriers; the absolute field magnitudes are those chosen for our particular design. The thermal barrier concept requires that the uniform field in the central cell rise sharply at both ends to a peak, in this case $12 \mathrm{~T}$, followed by a minimum point at $10 \%$ of this value, i.e., 1.2 T. This magnetic field profile is provided by a high field solencid and a gap separating it from the outer group of plug and transition coils. The length of the barrier cel1 between the 12-T peak and the inner mirror point of the plug is $14.3 \mathrm{~m}$. The plug coils are required to give an axial mirror ratio of 1.5 and also a positive radial well. These requirements are satisfied by means of a yin-yang coil pair. The yin-yang distorts the plasma cross section into an elliptical shape. In consequence, we have provided transition cee coils on both sides of the yin-yang in order to restore circularity. A primary constraint to the coil design is the need for magnetohydrodynamic (MHD) stability. This system has been designed to achieve interchange stability (which significantly affected the design of the transition coils). The arerchange stability calculation with the final transition coil yielded an upper limit of 1.38 for the central cell-to-plug $B$ ratio.

The only coil with a high field requirement and hence a high conductor field $(12.5 \mathrm{~T})$ is the barrier solenoid. This is therefore the only coil for which niobium-tin superconductor is required. It is also the on ly coil for which structural zequirements are here considered; the other coils should not present any difficulty in this regard.

In the following paragraphs, the several coils are discussed individually. 


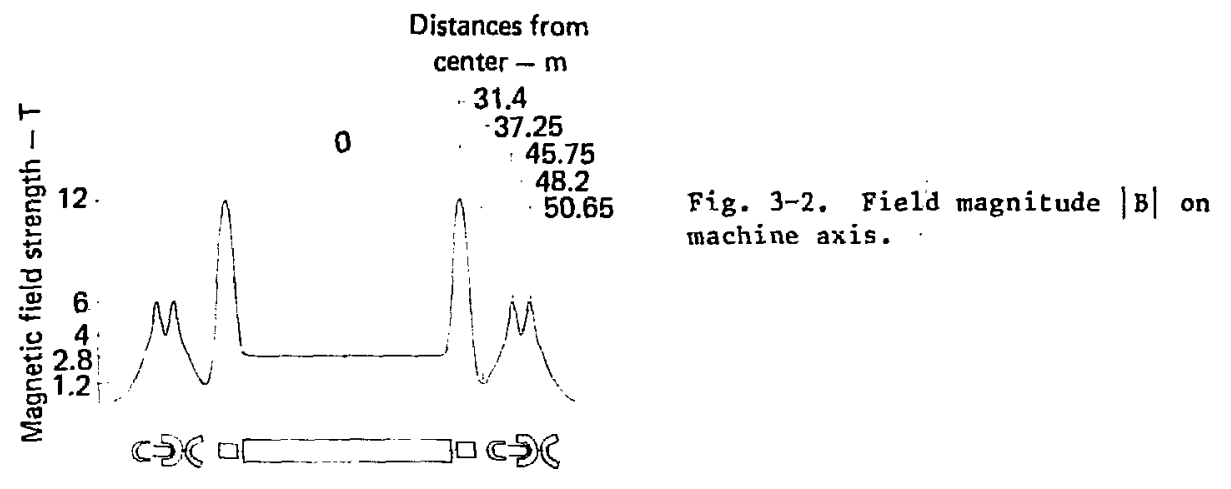

CENTRAL CELL

Figure 3-1 shows the central cel1 magnet represented as a continuous solenoid $56 \mathrm{~m}$ in total length (actua11y it will have discrete short solenoids accupying about $30 \%$ of the total cylindrical surface-see the section on Central Cell Design, Chapter 8 ). The inside coil radius for this magnet model is $2.6 \mathrm{~m}$, and the centerline field is $2.8 \mathrm{~T}$.

BARRIER COIL

This coil is required to give a peak field of $12 \mathrm{~T}$ on its centerline. It is a solenoid having an inside radius of $1.3 \mathrm{~m}$, a length of $4.5 \mathrm{~m}$, a radial thickness of $0.46 \mathrm{~m}$, and a current densicy of $24.3 \mathrm{MA} / \mathrm{m}^{2}$. The conductor volume is $19.9 \mathrm{~m}^{3}$. The inside radius accommodates approximately $0.7 \mathrm{~m}$ of shielding around the plasma. The length and thickness were chosen to give a high magnetic efficiency $(96 \%)$ so that the peak field in the conductor is only 12.5 T. This efficiency is expensive in terms of conductor volume, but the conductor field is the controlling factor, being at the limit of current niobium-tin superconductor development with ordinary liquid helium coolant. 
The conductor field is a maximum only at the inner radius-it falls off rapidly as the radius increases. Figure 3-3 shows a contour plot of the magnitude of the magnetic field $|B|$ for a section through the coil. The outer (radial) two-thirds of the section experiences fields of less than 8 T. Thus only the inner third of the coil requires niobium-tin superconductor. The coil would be built in two layers, with a supporting ring between them.

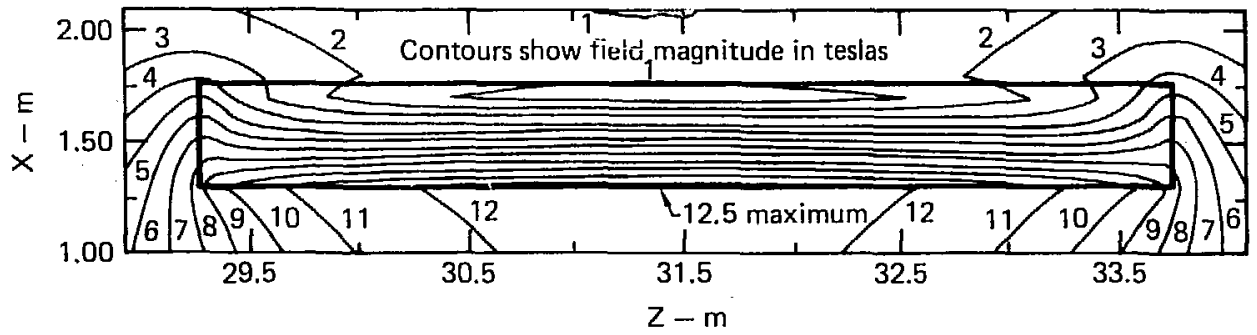

Fig. 3-3. Section through barrier coil showing contours of field magnitude $|B|$.

The upper curve in Fig. 3-4 shows the average radial force per unit of conductor volume plotted against the radius; it is approximately linear. If we ignore the hoop stress in the conductor and assume that the radial force is taken entirely by the supporting ring, the radial stress in the niobium-tin layer of the conductor is given by the lower curve. Figure 3-4 also shows the radial variation of the conductor field at the midplane of the coil. At a radius of approximately $1.45 \mathrm{~m}$, the conductor field has decreased to $8 \mathrm{~T}$. This radius defines the location of the supporting ring. The radial compressive stress at this point is approximately $32 \mathrm{MPa}$ ( 4640 psi), which is an acceptable stress in the conductor. The niobium-tin superconductor will be in a matrix of copper. The strain in copper $\left(\mathrm{E}=117 \times 10^{3} \mathrm{MPa}\right)$ at the above stress is only $0.027 \%$. If we assume that the support ring is made of a steel with a working stress of about 100,000 psi at cryogenic temperature, its 


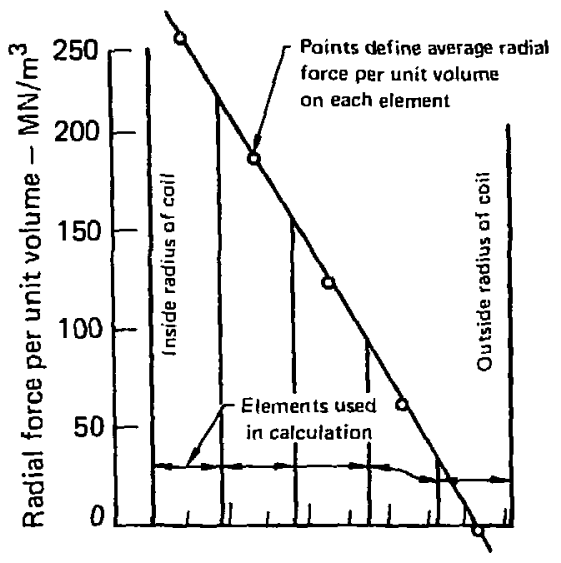

Fig. 3-4. Radial body force and stress in barrier coil.

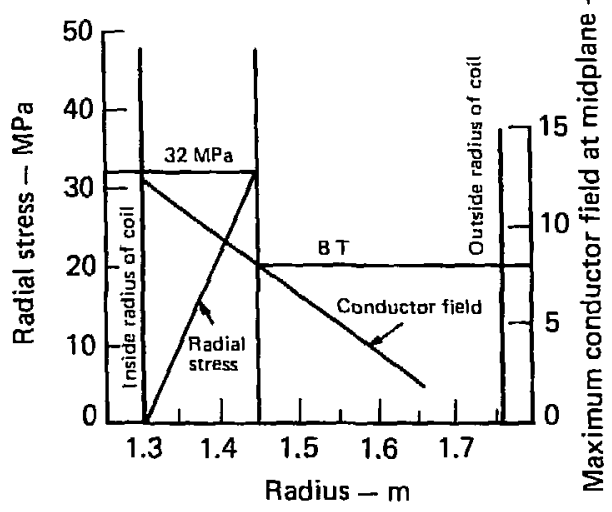

thickness needs to be only $\sim 0.07 \mathrm{~m}$, and $i$ ts tensile strain is $\sim 0.33 \%$. The same strain would occur in the superconductor. The combined strain in the superconductor is well within the limit for niobium-tin $(\sim 0.6 \%$ with no degradation in current carrying capacity ${ }^{3-2}$. If the hoop stress in the conductor were taken into account, the support ring could be significantly thimer.

Improved plasma performance would be obtained if the field in the barrier coil were even larger-this improved performance could be achieved by designing the niobium-tin superconductor to operate at lower temperature through the use of superfluid helium. 
The plasma within the cencral cell and the barrier coil is of circular cross section. If this plasma fiere allowed to enter the plug yin-yang directly it would be distorted to an elongated (elliptical) shape. The transition coil elongates the plasma in the opposite (perpendicular) direction, thus opposing the subsequent effect of the yin-yang and enabling the plasma to return to an approximately circular section at the center of the latter. The distortion is illustrated in Fig. 3-5, in which vacuum field lines are shown plotted in two perpendicular planes (both of these field lines begin at a radius of $1 \mathrm{~m}$ at the center of the reactor).
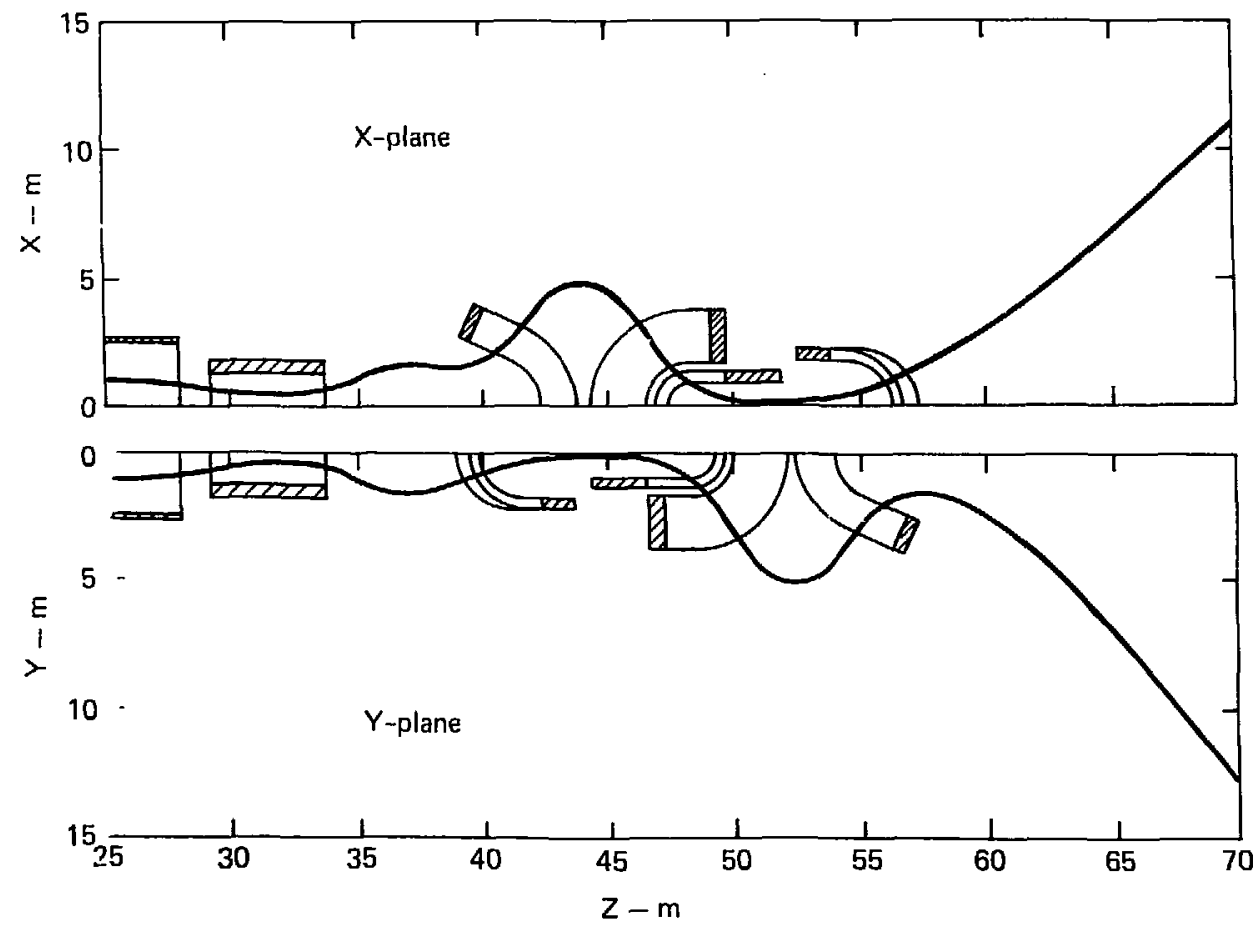

Fig. 3-5. Line plots through outer part of machine. 
The field shaping requirement of the transistion coil can be achieved by four axial conductors, one in each quadrant, with adjacent quadrant currents in opposite directions; i.e., Ioffe bars. In practice, the axial currents ran be provided by a cee coil. The minimu total current would result with a $180^{\circ}$ cee coil; however, several additional factors iffect the design of the coil. It must be close to the plug yin-yang and produce a significant axial field in order to keep the elliptical excursion of the flux bundle within reasonable l1mits (if the latter extends too far beyond the coils the ends of the ellipse spread into a dog-bone shape). On the other hand, the cee coil must not contribute more than a small proportion of the total field within the yin-yang; otherwise the latter will not develop its necessary magnetic well. These complications (and more) are quantified by the requirement for MHD interchange stability, in which if:

$$
\begin{aligned}
& x, y=\text { field line distances from axis, } \\
& \ddot{x}, \ddot{y}=\text { curvatures }\left(\frac{d^{2} x}{d s^{2}} \text { and } \frac{d^{2} y}{d s^{2}}\right), \\
& p \quad=\text { plasma pressure, } \\
& |B|=\text { magnitude of magnetic field; }
\end{aligned}
$$

then the integra1:

$$
\int \frac{p(x \ddot{x}+y \ddot{y})}{|B|} d s \text {, }
$$

taken along a field line close to the machine axis and between the two outer mirror points is required to be $\geq 0$. With reference to Fig. $3-5$, the field line excursion that occurs in the $x z$ plane between the transition and yin-yang coils has a small value of $|B|$, a large value of $y$, and a large negative curva-ure; the field line excursion therefore makes a major negative contribution 
to the above integral. The magnitude of the negative effect is determined by the geometry of the transition coil. In our design, the major arc angle of the coil has been roughly optimized at $130^{\circ}$. The reduced angle necessitates a larger current (and a bulkier coil) in order to maintain the required quadrupole effect, but thereby increases the local axial component of the field sufficiently to produce the required stability.

The transition coil is separated from the barrier coil sufficiently to give a minimum field on centerline in the barrier region of $1.2 \mathrm{~T}$.

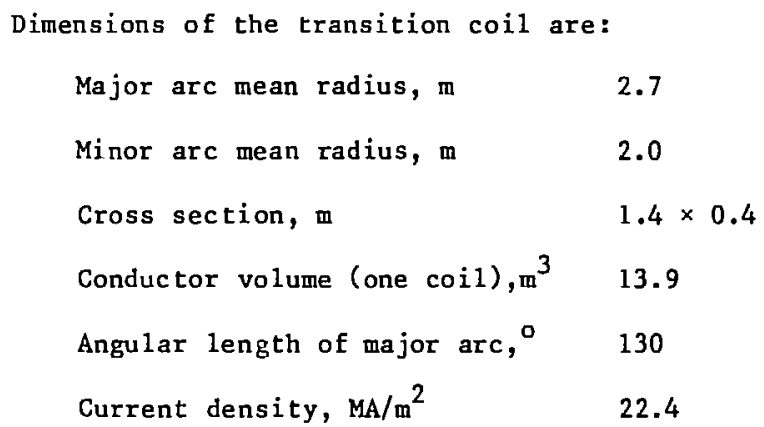

Niobium-titanium superconductor will be used; the maximum conductor field is s1ightly over 6 T.

PLUG YIN-YANG COILS

The plug coils are specified to have an on-axis central field of $4 \mathrm{~T}$ and a mirror field of $6 \mathrm{~T}$ (hence, a vacuum mirror ratio of 1.5 ). They must a1so have a radial magnetic well to provide the "anchor" for MHD stability. This requirement led to the choice of a full $180^{\circ}$ yin-yang.

Each half of the gin-yang consists of three layers of equal thickness; these are separated in the minor arc portion of the coils. The purpose of this separation is to reduce the average current density and thereby the magnitude of the field within the conductor (the miximum norma1ly occurs on the 
inside of the 'minor arc). For the same reason, the conductor section is relatively deep and narrow. A maximum conductor field of $9.0 \mathrm{~T}$ was calculated, giving a magnetic efficiency of $66 \%$. Contours of $|B|$ are shown in Fig. 3-6 for a section of the yin-yang in the yz plane. Further refinement of the design (by using still deeper and narrower sections and/or more spreading of the conductor in the minor arc) should permit the reduction of the maximum conductor field to the specified $8 \mathrm{~T}$. Niobium-titanium superconductor will be used. The following are the dimensions of the coils:

$$
\begin{array}{ll}
\text { Mean radius of major arc, m } & 2.7 \\
\text { Mean radii of minor arcs, m } & 1.06,1.18 \text {, and } 1.30 \\
\text { Depth of conductor section, m } & 2.1 \\
\text { Thickness of each of } 3 \text { Iayers } & 0.12 . \\
\quad \text { of conductor section, m } & 40.9
\end{array}
$$

The center and outer layers have short straight sections between the major and minor arcs in order to give the required $0.04-m$ separation between layers. These dimensions permit a minimum shielding thickness around the plaswa of approximately $0.8 \mathrm{~m}$ at the corner of the section.

The current density is $22.8 \mathrm{MA} / \mathrm{m}^{2}$ for each half of the yin-yang. Figure 3-7, a transverse section at the center of the plug, illustrates the magnitude of the radial magnetic well.

CIRCULARIZING COIL

This coil performs a function similar to that of the transition coil. The circularizing coil converts the elliptical magnetic flux bundle leaving the plug to an approximately circular cross section, thus facilitating the 


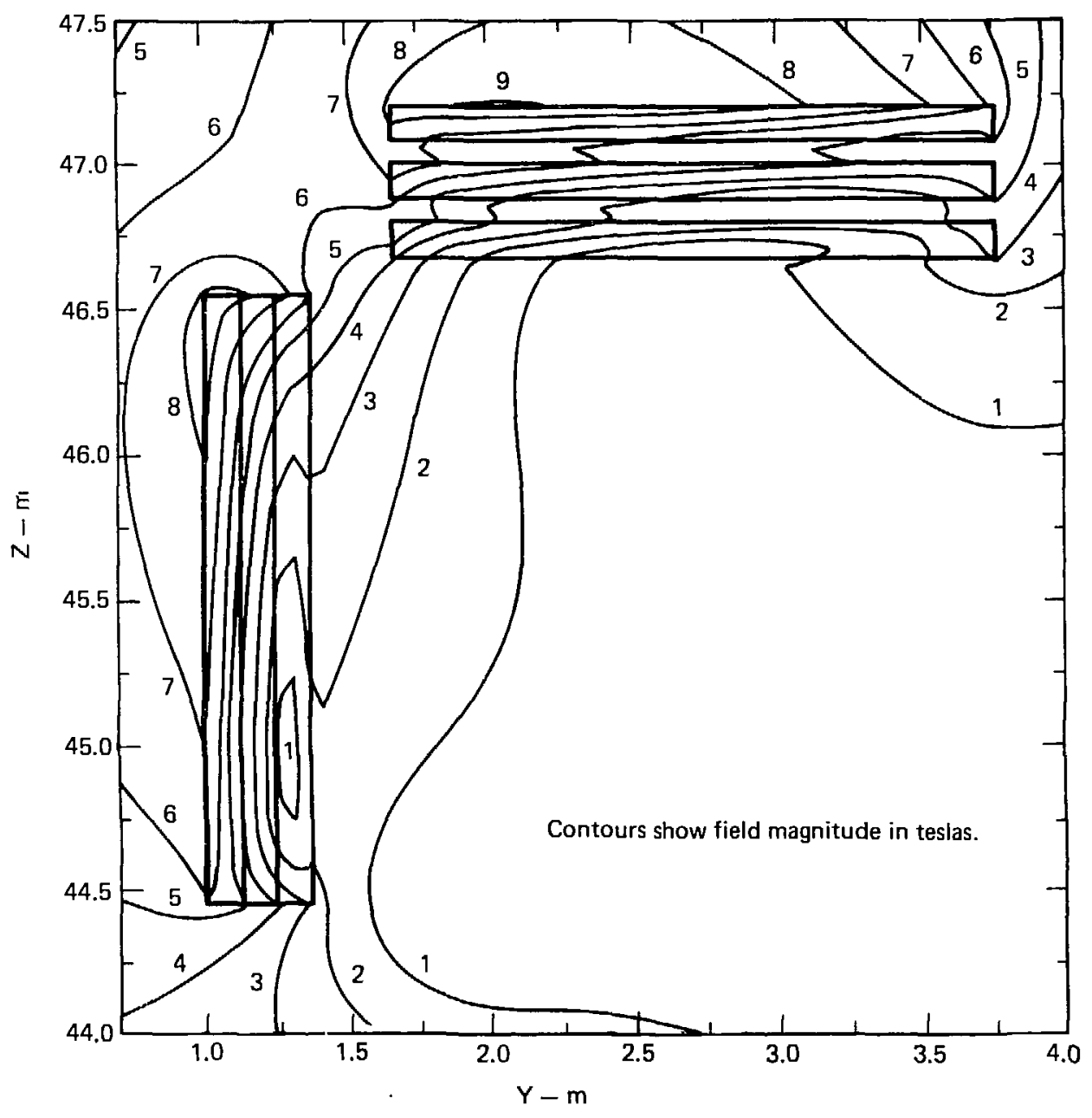

Fig. 3-6. Section through plug yin-yang coils showing contours of field magnitude $|\mathrm{B}|$. 


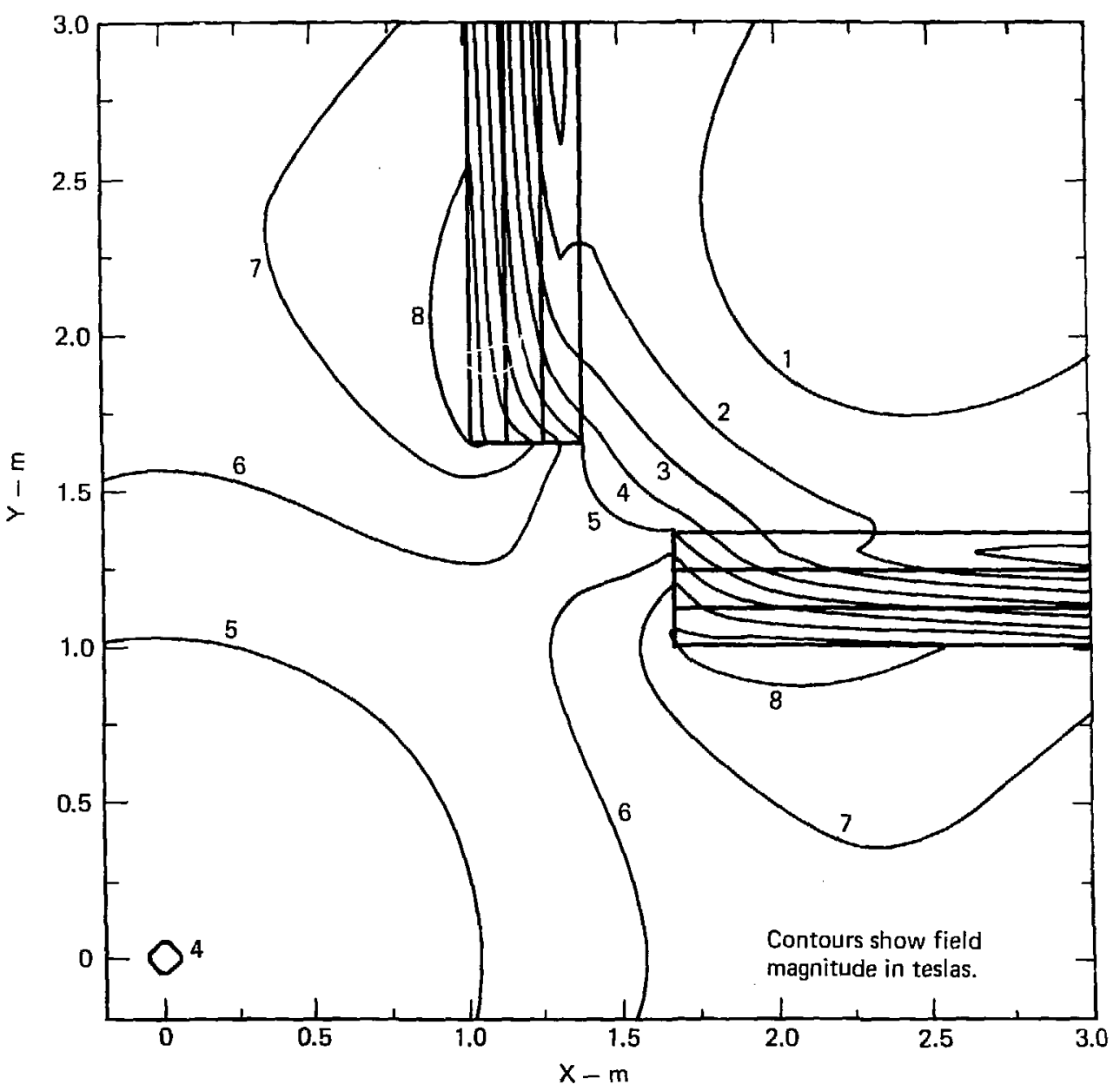

Fig. 3-7. Transverse section through center of plug ( $z=48.2 \mathrm{~m}$ ) showing contours of field magnitude, and illustrating radial well. 
design of the plasma direct converter. For calculational convenience the coil has been made identical to the transition coil (only the orientation is different). Making the coii identical is not strictly necessary, however, since the stability criterion does not involve the circularizing coil. A full $180^{\circ}$ major arc cee coil wou d be equally effective and would have a smaller conductor volume.

$\begin{array}{lc}\text { The following are the dimensions of the circularizing coil: } \\ \text { Mean radius of major arc, m } & 2.7 \\ \text { Mean radius of minor arc, m } & 2.0 \\ \text { Conductor cross section, m } & 1.4 \times 0.4 \\ \text { Conductor volume (one coil), } \mathrm{m}^{3} & 13.9 \\ \text { Angular length of arc, o } & 130 \\ \text { Current density, MA/ }{ }^{2} & 22.4 \\ \text { Superconductor material } & \text { Niobium-titanium }\end{array}$

\section{ALTERNATIVE REACTOR DESIGN WITH SOLENUIDAL PLUG COILS}

For comparison purposes, a magnet set has been designed in which the plug is not required to have a radial well. (In this more speculative design the "anchor" for MHD stability must be provided by some other means, such as a hot electron ring in the plug, i.e., an EBT cell; we have not yet quantified the stability requirement in such a case.) This alternative design permits the plug coils to be merely a pair of short solenoids giving the required axial mirror ratio. There is no elliptic distortion of the plasma and therefore no need for either the tra.sition or circularizing coils. The result is a considerable reduction in the length of the reactor, and in the volume (and hence cost) of the conductor. The overal1 length of the plug and barrier coil set is reduced from 28 to $16.3 \mathrm{~m}$. The length of the barrier 
region (from the barrier peak field to the first plug mirror) is reduced from 14.3 to $9.3 \mathrm{~m}$. This reduction would considerably improve the reactor performance because the barrier "pump" beam power is proportional to the volume of the barrier region. The conductor volume for the plug coils is reduced from $40.9 \mathrm{~m}^{3}$ for a yin-yang pair to on $1 y 14.3 \mathrm{~m}^{3}$ for a solenoidal pair. The conductor volume reduction is even more dramatic because the alternative design has no transition or circularizing coil-a combined volume of $27.8 \mathrm{~m}^{3}$. Thus, the total reduction is from 68.7 to $14.3 \mathrm{~m}^{3}$, a factor of almost 5 . The peak conductor field goes from 9.0 to $7.9 \mathrm{~T}$. The following are the actual dimensions of the two plug solenoids: inside radius, $1.5 \mathrm{~m}$; radial thickness, $0.34 \mathrm{~m}$; length, $2.0 \mathrm{~m}$; cument densities, 24.6 and $25.0 \mathrm{MA} / \mathrm{m}^{2}$. Figure $3-8$ shows the magnitude of the magnetic field plotted along the axis of the machine (this figure should be compared with the corresponding Fig. 3-2 for the normal design).

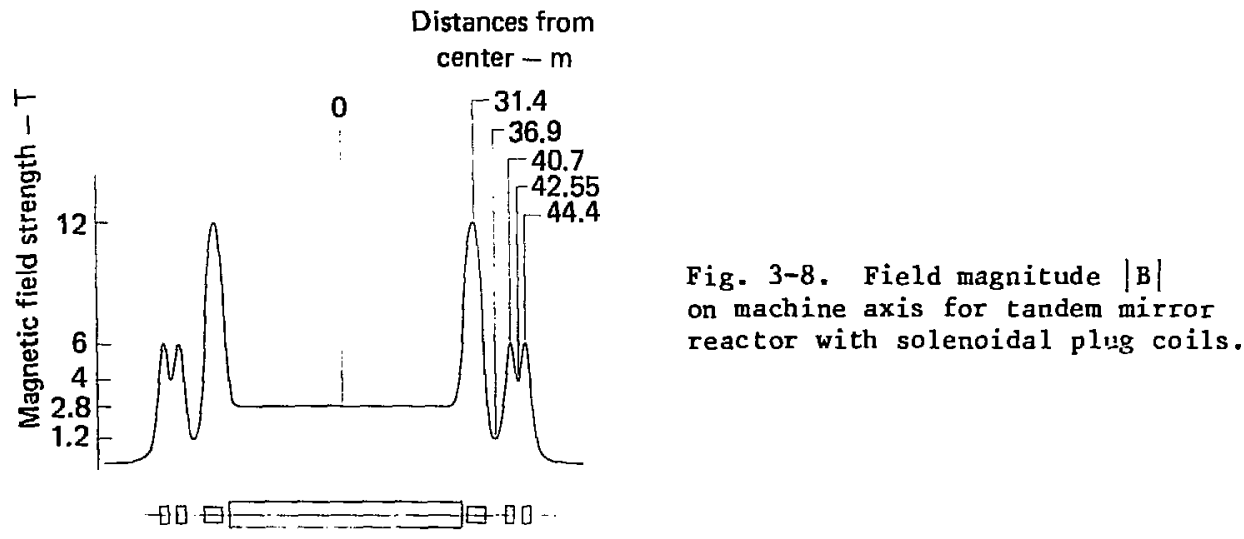




\section{REFERENCES}

3-1. S. J. Sackett, EFFI-A Code for Calculating the Electromagnetic Field, Force and Inductance in Coil Systems of Arbitrary Geometry, Lawrence Livermore Laboratory, Livermore, CA, UCRL-52402 (1978).

3-2. D. W. Deis, D. N. Cornish, A. R. Kosdahl, and D. G. Hirzel, Mechanical Strain of Large, Multifilament $\mathrm{Nb} 3 \mathrm{Sn}$ Conductors for Fusion Magnets, Lawrence Livermore Laboratory, Livermore, CA, UCRL-78890 (1977). 
G. W. Hamilton, J. L. Erickson, J. H. Fink

Introduction .............. 83

Neutral-Beam Specifications . . . . . . 85

Ion Source ................ 86

Double-Charge-Exchange Cell . . . . . . . 91

400-kV Acceleration Column ........... . 96

Insulation Problems . . . . . . . . 102

High-Voltage Protective Circuit . . . . . . 103

Voltage Regulation ......... 107

Metal Vapor Stripping Cell . . . . . . . . 107

Disposal of Charged Beams . . . . . . . . 110

Vacuum Pumping and Drift Duct Blocking. . . . . . 111

Protection Against Neutron, Microwave, and Synchrotron Radiation . . . . . . . . 115

References ................ 119 
Neutral beam requirements for the end plugs of a Tandem Mirror Reactor $(T M R)^{4-1}$ have been greatly reduced by the increased plug electron temperature made possible by thermal barriers and by electron cyclotron resonance heating (FCRH). In this version neutral beams in the end plugs are required only to maintain the density of fast $\mathrm{H}^{+}$, for which the loss rate is low because of the reduction of electron drag. The neutral-beam requirement for each plug of the TMR is now only $0.25 \mathrm{MW}$ of 400-keV $\mathrm{H}^{0}$ trapped in the plasma. Several changes in viewpoint are possible because of the reduced rer quirements. One change is the fact that it is not essential to have a high trapping fraction of the injected neutrals to have a favorable power balance, making it possible to reduce the density and size of the plasmas in the end plugs, with resulting economies in the magnets and structure. Another change is the fact that the neutral-beam design can be conducted with only a few extrapolations beyond existing technology. It is possible to be rather specific about the technical developments required for such a system to become operational.

As part of an operating system, the injectors must be conpact, operate at better than $50 \%$ efficiency, and require scheduled maintenance shutdown not more than once a year. These injectors are estimated to cost about $\$ 1.00 / \mathrm{W}$, injected ${ }^{4-2}$.

While such injectors seem advanced with: respect to today's low duty cycle, short pulse, $120-\mathrm{keV}_{\text {beams }}{ }^{4-3}$, their development is consistent with today.'s seam programs. Our need for beams of longer pulse length-about $1.5 \mathrm{~s}$ in the Tokamak Fusion Test Reactor (TFTR) or $30 \mathrm{~s}$ in the Magnetic Fusion Test Facility (MFTF) -and for beams delivering higher energy will lead to progress 
that should make the TMR injector an acceptable engineering goal within the next decade.

In the process of designing such units, some of the problems encountered involve more than filling in "engineering details." They call for good judgment when available data are inadequate. In some cases ingenious inventions are needed to meet the specific requirements of the support structure, or perhaps the beam alignment, heat transfer, circuitry, continuous pumping, radiation hardening, magnetic shielding, safety, etc. We believe that engineers can be relied upon to provide satisfactory answers to such questions. However, before the design of the TMR neutral-beam injector can be started, the feasibility of the overall design objectives must be demonstrated.

Fortunately, these developments are either an outgrowth of the positive ion program or are related to it. Thus the initial development efforts to demonstrate feasibility need not be very expensive, nor need they radically alter present beam programs. However, subsequent efforcs, such as TETR upgrade or MFTF-B, that utilize these concepts will incur large costs and some engineering risk. Getting the first injector operational may require as much as $\$ 3 / \mathrm{W}$.

Figure 1-5 is an overall view of the 400-keV injector assembly we will describe to show how the TMR injection requirements may be met. Figure 4-1 is a schematic of the injector. The injector consists of a low-energy source of $\mathrm{H}^{-}$ions, a 400-kV acceleration column, a vapor stripping cell to convert the $\mathrm{H}^{-}$ions to $\mathrm{H}^{0}$, a charged beam disposal section, and the systems required for high-voltage power and protective circuits, voltage insulation, gas pumping, and radiation protection. Each of these components will be discussed in the following sections. 


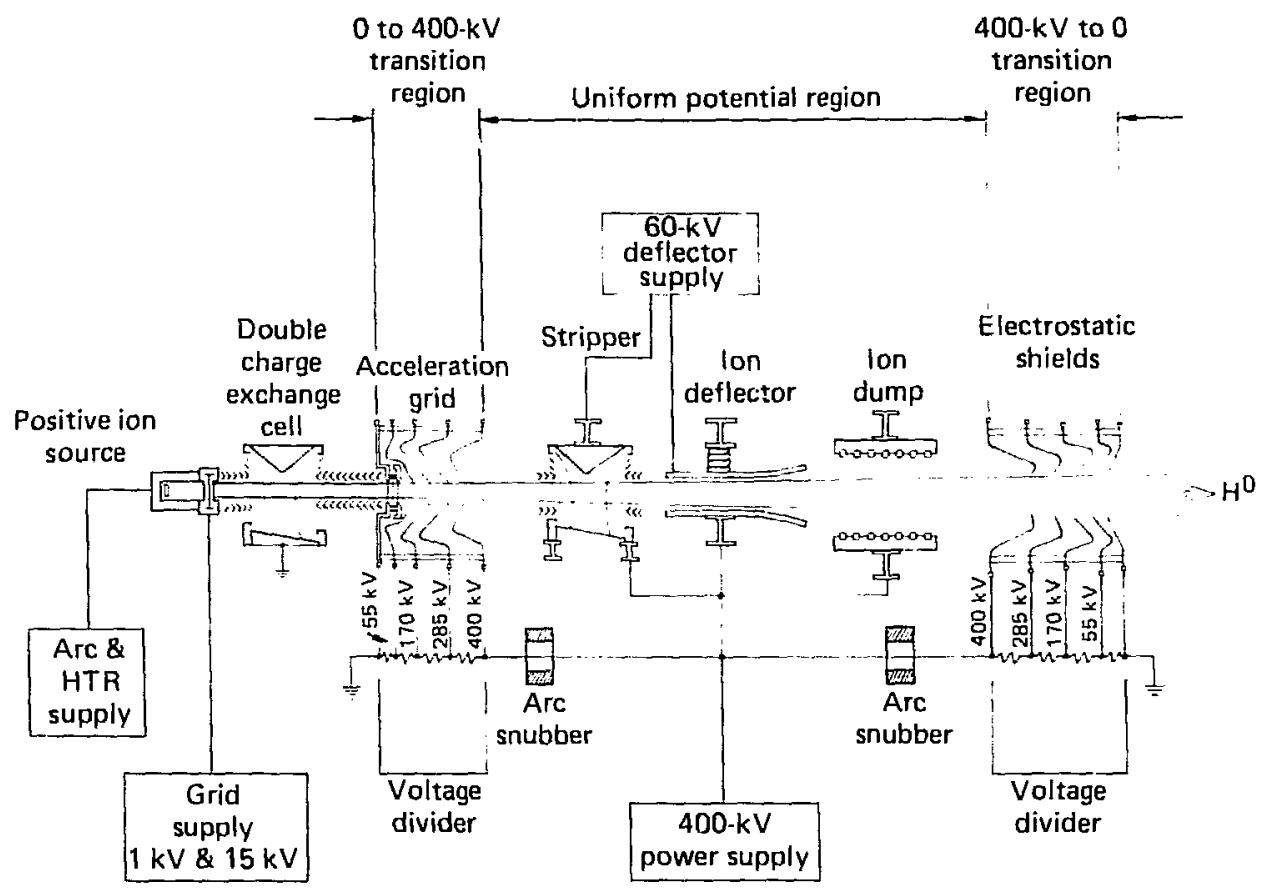

Fig. 4-1. Components of 400-keV injector.

NEUTRAL-BEAM SPECIFICATIONS

The specifications for the neutra1-beam system are determined by the basic requirement for $0.25 \mathrm{~W}$ trapped in each end-plug plasma at $400 \mathrm{keV}$. We have chosen $\mathrm{H}^{0}$ rather than $\mathrm{D}^{0}$ or $\mathrm{T}^{0}$ for this system to reduce the gyroradius and thereby to improve the DCLC plasma stability criterion. This choice is also convenient to reduce the neutron radiation from the end-plug plasma. The neutralization efficiency for negative ions is independent of the choice of isotope. 
Table 4-1 indicates the specifications of the components of the $400-\mathrm{keV}$ neutral-beam system calculated from the TMR requirements, for a design using negative ions produced by double-charge-exchange and neutralized by a vapor stripping cell optimized for maximum $\mathrm{H}^{0}$ output. No attempt is made for electrostatic direct energy conversion of the residual charged beams. Thermal energy recovery of the 1.9 MW deposited on beam dumps is a possibility. We have considered other options for each component of the injection system. These considerations will be discussed in the following sections.

ION SOURCE

The fundamental component of a neurral-beam injector is the ion source. If an injector is to be efficient and reliable, and to operate continuously for at least one year, its ion source must be capable of such performance. To adapt today's positive ion sources to meet the goals of the future, considerable development is required. Progress must be made to ensure extended cathode life, to provide grid cooling, and to form beams that are 80 or $90 \%$ atomic ions.

To achieve operating efficiencies of $50 \%$ or better with hydrogen at energies in excess of $75 \mathrm{keV}$ or deuterium at energies greater than $150 \mathrm{keV}$, the neutrals must be formed from high-energy negative ions instead of from positive ions as is the current practice. This is because the charge-exchange cross section of negative ions is large and relatively independent of the beam energy. At present there are several types of negative ion sources under development. 
TABLE 4-1. Neutra1-beam performance specifications.

\begin{tabular}{|c|c|c|c|c|}
\hline Item & Efficiency & $\begin{array}{c}\text { Voltage } \\
\text { (kev) }\end{array}$ & $\begin{array}{l}\text { Current } \\
\text { (A) }\end{array}$ & $\begin{array}{l}\text { Power } \\
\text { (MW) }\end{array}$ \\
\hline $\begin{array}{l}\mathrm{H}^{0} \text { trapped in plasma, } \\
\text { each plug }\end{array}$ & -- & 400 & 0.625 & 0.25 \\
\hline$\stackrel{\text { Trapping }}{=n_{p}} \frac{\text { fraction }}{\mathrm{T}^{v} / \mathrm{v}_{B}}$ & \multicolumn{3}{|c|}{ Trapping fraction } & \\
\hline$H^{0}$ injected into plasma & -- & \multirow[t]{2}{*}{400} & \multirow[t]{2}{*}{3.205} & \multirow[t]{2}{*}{1.282} \\
\hline $\begin{array}{l}\text { Neutralizer efficiency } \\
\text { (optimized metal vapor } \\
\text { stripping cell) }\end{array}$ & 0.60 & & & \\
\hline $\mathrm{H}^{-}$beam from accelerator & -- & 400 & 5.342 & 2.137 \\
\hline Power loss in accelerator & - & 400 & 0.33 & 0.132 \\
\hline Power loss in voltage divider & - & 400 & 0.10 & 0.040 \\
\hline $\begin{array}{l}\text { Transparency of electron } \\
\text { suppressor before } \\
\text { acceleration }\end{array}$ & 0.80 & & & \\
\hline $\mathrm{H}^{-}$beam produced & - & 1 & 7.09 & 0.007 \\
\hline \multicolumn{5}{|l|}{$\begin{array}{l}\text { Double charge exchange } \\
\text { conversion efficiencies - }\end{array}$} \\
\hline from $1-\mathrm{keV} \mathrm{H}^{+}$ & \multicolumn{4}{|c|}{$\begin{array}{l}0.20 \text { (net after electron detachment in gas } \\
\text { between Cs vapor and accelerator) }\end{array}$} \\
\hline from $\mathrm{H}_{2}^{+}$and $\mathrm{H}_{3}^{+}$ & \multicolumn{4}{|c|}{$\begin{array}{l}0 \text { (assuming no dissociation of low-energy } \\
\text { molecular ions) }\end{array}$} \\
\hline
\end{tabular}

Composition of 1-keV beamm-

$$
\begin{array}{ll}
\mathrm{H}_{1}^{+} & 0.80 \\
\mathrm{H}_{2}^{+} \text {and } \mathrm{H}_{3}^{+} & 0.20
\end{array}
$$

$\begin{array}{llllll}1-\mathrm{keV} \text { positive beam } & -- & 1 & 44.3 & 0.044\end{array}$

Extractor grid transparency $\quad 0.5$

Ion source current density $\quad 0.25 \mathrm{~A} / \mathrm{cm}^{2}$ at plasma

Area of extraction grids $=$

$44.3 /(0.5 \times 0.25)=354 \mathrm{~cm}^{2}=7 \times 50 \mathrm{~cm}$ 
TABLE 4-l. (cont.)

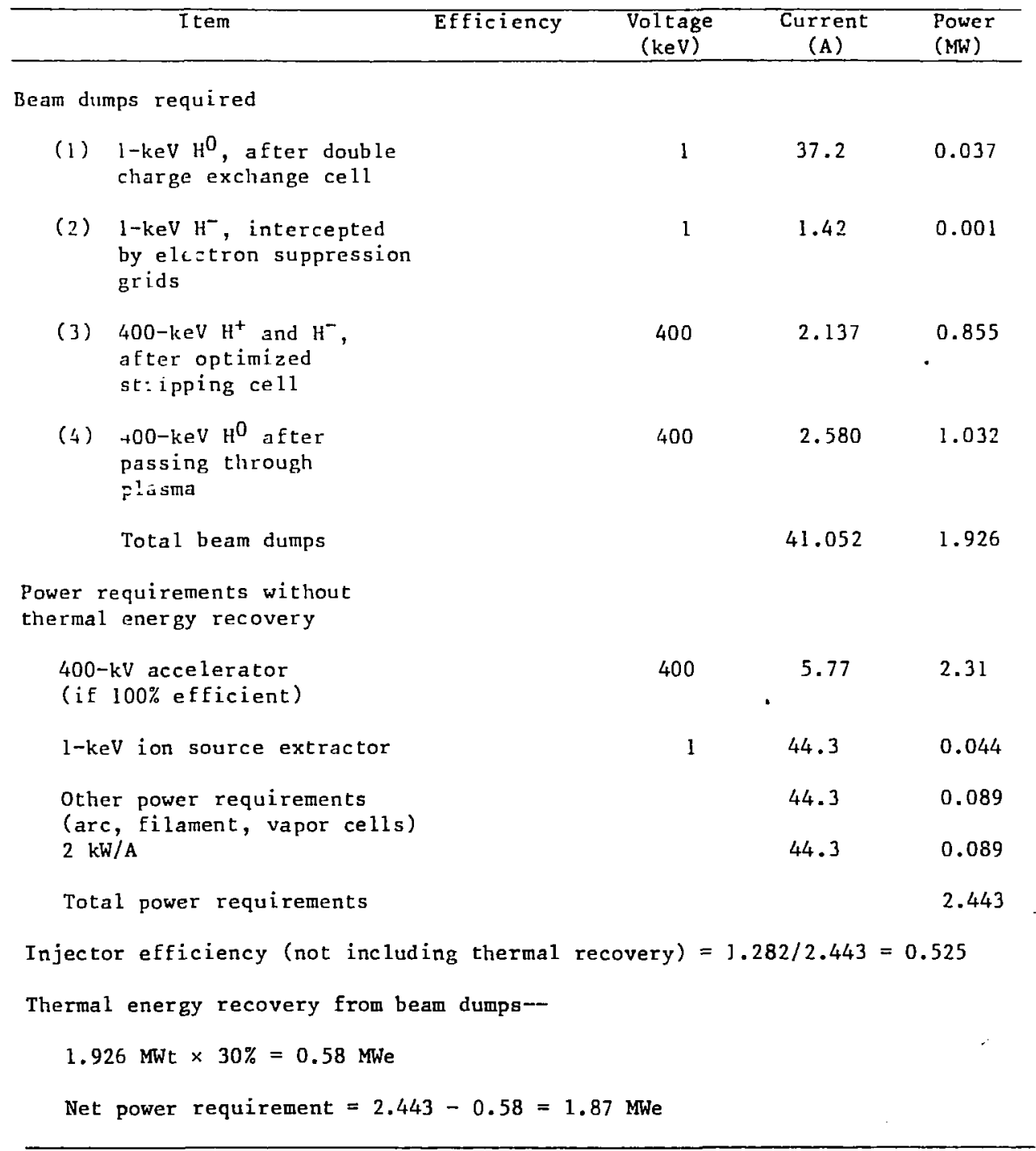


One approach to source design produces negatives from posilives by means of a metal-vapor double-charge-exchange cell. Approximately $20 \%$ of an incoming positive ion beam at $1 \mathrm{keV}$ becomes negative in a cesium vapor cell of proper design. The remaining $80 \%$ of the beam leaves the cell as unwanted low-energy neutrals. To make this process functional a positive ion source is needed that can continuously deliver $1-k e V$ ions that are 80 or $90 \%$ atomic. This requirement is very similar to that of the ongoing positive ion program. However, in this case the desired beam energy is much lower. As a consequence, the ion source needed for the double-charge-exchange method of forming negative ions will be a by-product of the present neutral-berm program.

A second approach to negative ion source design uses direct extraction of $\mathrm{H}^{-}$produced in a plasma by bombardment of surfaces with low work function. A transverse magnetic field is required to separate the extracted $\mathrm{H}^{-}$ from the plasma electrons. Both approaches have assets and liabilities; we are choosing the double-charge-exchange approach for the TMR design study because the requirements are straightforward and because we expect a somewhat higher attainable current density per unit length of a long module.

Figure 4-2 is a drawing of our concept of a continuously operating source of l-keV atomic hydrogen ions $\left(\mathrm{H}_{1}^{+}\right)$. We show a large-area longlife cathode, which could be made of lanthanum hexaboride, supporting $10 \mathrm{~A} / \mathrm{cm}^{2}$ of thermionic electron emission. The walls of the arc chamber are covered with magnets to form magnetic cusps called a picket fence. This configuration reduces the rate of ion recombination at the walls and thereby increases the yield of atomic ions.

The extracted low-energy beam current is $0.87 \mathrm{~A}$ per linear centimetre of a long plasma source. This current requires about $87 \mathrm{~A}$ of thermionic emission per centimetre-that can be provided by $9 \mathrm{~cm}^{2}$ of emission surface per linear centimetre. 


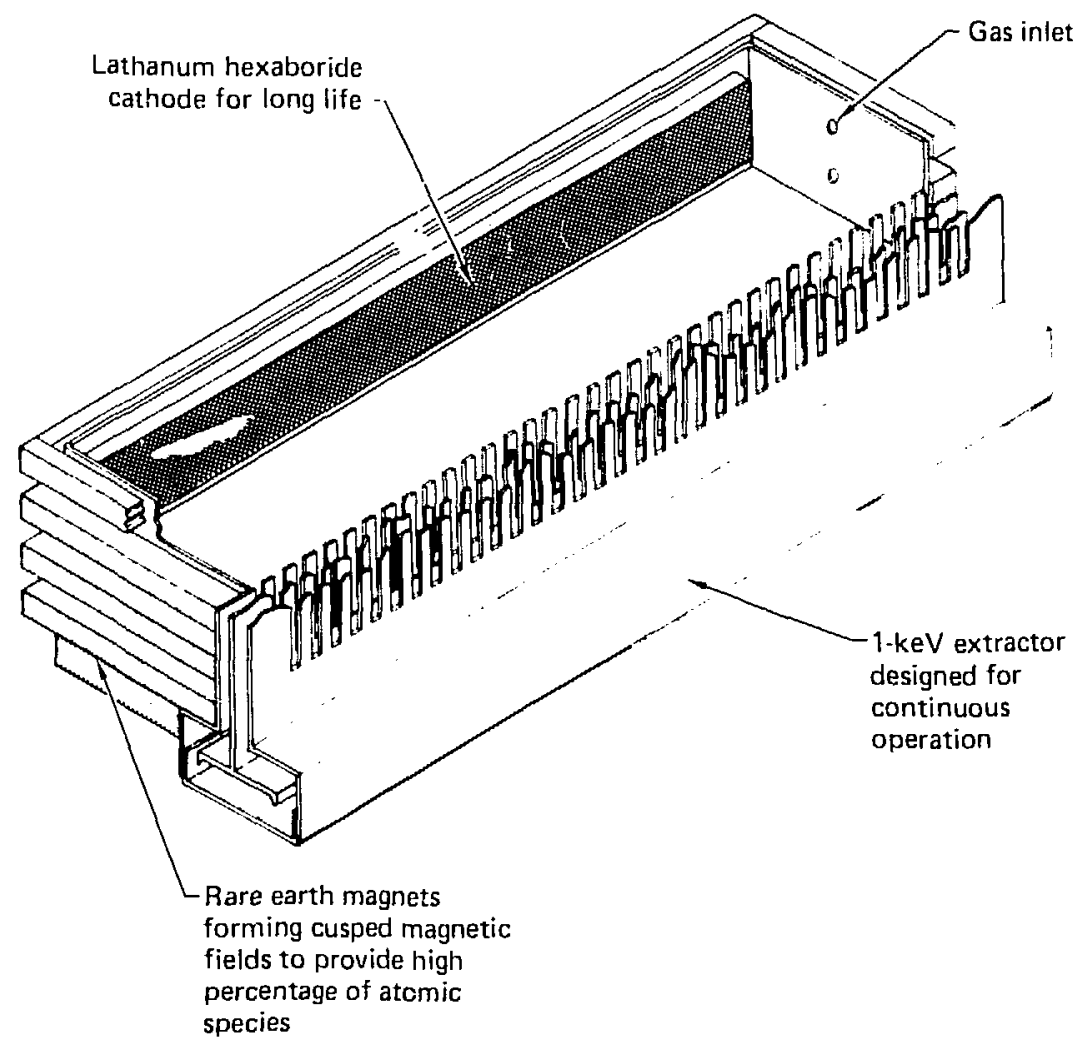

Fig. 4-2. Low-energy $\mathrm{H}^{+}$ion source for $\mathrm{H}^{-}$production by double-charge-exchange.

To provide continuous operation, a $1-\mathrm{keV}$ extractor grid is required to remove the energy dissipation resulting from ion bombardment of the grid rods. A proposed extractor ${ }^{4-4}$ has been studied using conduction-cooled grid rods. This extractor is capable of dissipating a power loading of $40 \mathrm{~W} / \mathrm{cm}^{2}-$ adequate for the purpose. 
Experiments now being performed at Lawrence Berkeley Laboratory ${ }^{4-5}$ and in the Soviet Union ${ }^{4-6}$ are producing negative ions by passing a positive ion beam through a plume of sodium vapor. The plume is produced in a cell that confines the metal vapor while allowing the beam to pass.

Of the several types of nozzles that could be considered for producing the plume, we have chosen a plug-type nozzle. ${ }^{4-7}$ As shown in Fig. 4-3, the vapor is collected in manifolds on both sides of the nozzle. The vapor undergoes a Prandtl-Meyer expansion around the edge of a slit with the free-jet direction limited by the background pressure in the cell. The boundary layer flow along the nozzle surface is trapped between two well-directed jets, thus eliminating any significant loss of material.

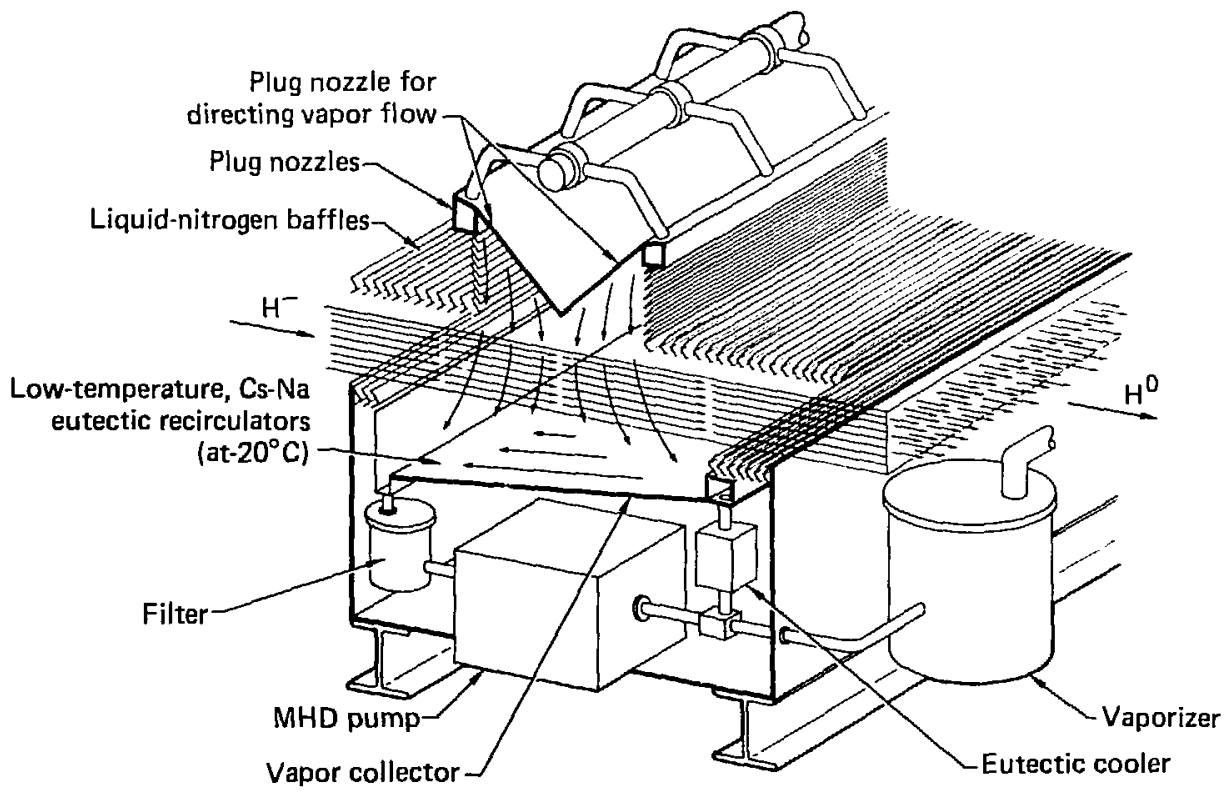

Fig. 4-3. Double-charge-exchange cell using cesium-sodium eutectic. 
The alkali metals are all capable of performing as double-chargeexchange targets for the positive beam. Present experimerts most often use sodium, but passing a l-keV positive through cesium or rubidium vapor produces a higher equilibrium fraction $(21 \%) .^{4-8}$ However, since the beam is at only $1 \mathrm{keV}$, it is more difficult to focus than the 7-keV beam that produces the maximum equilibrium fraction in sodium ( $12 \%$ ). We have chosen cesium as the material for this double-charge-exchange cell.

When the beam enters the cesium plume it is assumed to be $80 \% \mathrm{H}^{+}$and $20 \% \mathrm{H}_{2}^{+}$and $\mathrm{H}_{3}^{+}$. As the hydrogen ions collide with cesium atoms they capture an electron to become neutral, or capture two electrons to become negative. The cross sections for these two processes are designated $\sigma_{10}$ and ${ }_{1-1}$, respectively. Some of the neutral hydrogen atoms so formed can becone positives $\left(\sigma_{01}\right)$ or negatives $\left(\sigma_{0-1}\right)$ by further collisions. Eventually, the beam will reach an equilibrium state in which the charge-exchange processes are balanced. We assume that the $\mathrm{H}_{2}^{+}$and $\mathrm{H}_{3}^{+}$are not dissociated at this low energy and do not contribute to the $\mathrm{H}^{-}$production.

The thickness, $\pi$, of the vapor plume is its density, $n$, times the beam path length, $\ell$. The fractions of positive, neutral, or negative hydrogen atoms $\left(\mathrm{F}^{+}, \mathrm{F}^{0}, \mathrm{~F}^{-}\right)$at any given point along the path length are determined by the cross sections for the various atomic processes between I-keV hydrogrn and thermal cesium. A series of differential equations characterizes the situation:

$$
\begin{aligned}
& \mathrm{dF}^{+} / \mathrm{d} \pi=-\left(\sigma_{10}+\sigma_{1-1}\right) \mathrm{F}^{+}+\sigma_{01} \mathrm{~F}^{0}+\sigma_{-11} \mathrm{~F}^{-} \\
& \mathrm{dF}^{0} / \mathrm{d \pi}=\sigma_{10} \mathrm{~F}^{+}-\left(\sigma_{01}+\sigma_{0-1}\right) \mathrm{F}^{0}+\sigma_{-10} \mathrm{~F}^{-} \\
& \mathrm{dF}^{-} / \mathrm{d} \pi=\sigma_{1-1} \mathrm{~F}^{+}+\sigma_{0-1} \mathrm{~F}^{0}-\left(\sigma_{-11}+\sigma_{-10}\right) \mathrm{F}^{-}
\end{aligned}
$$


The solution ${ }^{4-9}$ of these equations results in Fig. 4-4. The maximum conversion from $\mathrm{H}^{+}$to $\mathrm{H}^{-}$occurs at a plume thickness, $\pi$, of greater than $2 \times 10^{15}$ atoms $/ \mathrm{cm}^{2}$. This is the design thickness for the double-chargeexchange cell.

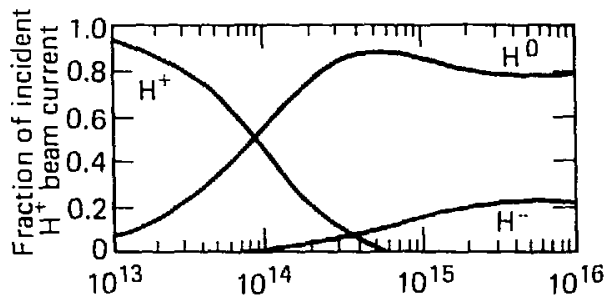

Fig. 4-4. Beam composition in cesium double-charge-exchange cell.

Target thickness - Cs atoms $/ \mathrm{cm}^{2}$

Molecular ions $\left(\mathrm{H}_{2}^{+}, \mathrm{H}_{3}^{+}\right)$entering the double-charge-exchange cell are a potential problem. At $1 \mathrm{keV}$, the optics of the ion beam are tenuous at best. If $1-k e V$ molecular ions dissociate in the cesium to become half or third energy components, the situation worsens. One current goal of the Lawrence Berkeley Laboratory source development program is to produce a positive ion beam that is $90 \%$ atomic. With this goal achieved, the beam can be transported efficiently through the cell.

The utmost concern when using a metal vapor cell in a high-voltage beam line is that all vapor be confined to the cell. The minutest coating of cesium on an insulator or an electrode will enhance field emission characteristics or provide enough of a conducting path to encourage breakdown. An additional concern is that cesium would become a contaminant in the reactor plasma.

Several features of the cell design serve to eliminate metal vapor 1oss. They include a flowing eutectic collector at $-20^{\circ} \mathrm{C}$, the plug nozzle design, and the venetian blind collectors surrounding the cell apertures. By taking these precautions, we limit the cesium flux from the cell to less than 
$10^{11}$ atoms $/ \mathrm{cm}^{2} / \mathrm{s}$. The loss of cesium during periodic shutdowns is prevented by shutter valves at each aperture. The valves guard against cesium loss both during routine maintenance and in the event of a sudden reactor shutdown.

The key to cesium vapor control is the liquid collector. The normal melting point of cesium is $28^{\circ} \mathrm{C}$. By forming a eutectic with sodium ree Fig. 4-5), the cesium can be contained as a liquid at as low as $-29^{\circ} \mathrm{C} .{ }^{4-10}$ Therefore, all collection of vapor once it has crossed the beamline can be made as a liquid at $-20^{\circ} \mathrm{C}$ rather than above the normal cesium melting point. Use of the eutectic results in a drop in vapor pressure of more than 2 orders of magnitude. Cesium could be collected as a solid at even lower temperatures with lower vapor pressures, but the problem of recirculating this solid cesium without heating and melting the surface is not a trivial one.

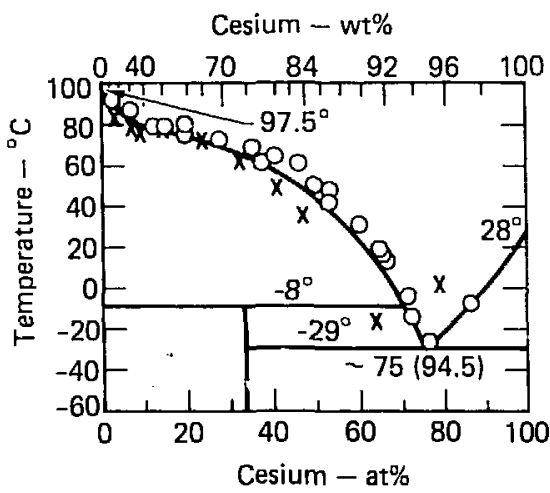

Fig. 4-5. Melting point depression for cesiur-sodium eutectic.

The vapor pressure of cesium is much higher than that of sodium. Thus when we boil the eutectic to produce the vapor plume, the plume is predominantly (99\%) cesium and performs the double-charge-exchange function as cesium. The potential for losing enough cesium to disrupt the viscosity of the flowing collector is very low. First, the quantity of cesium lost from 
the recirculating volume is very small due to the precautions discussed above. Second, at $-20^{\circ} \mathrm{C}$ (the operating point for the collector), the atomic percentage of cesium can vary from 73 to $81 \%$ without the eutectic solidifying. For these reasons, careful monitoring of the makeup in the eutectic will not be necessary. We propose an $80 \%$ (atomic percent) cesium-20\% sodium eutectic mixture for the doubie-charge-exchange cell. But since the vapor plume remains $99 \%$ cesium, the cell will produce a $20 \%$ double-charge-exchange efficiency for the $1-\mathrm{keV} \mathrm{H}^{+}$beam.

Al1 cesium-sodium eutectic is continuously recycled to permit extended beamline operation. As the flow leaves the collector it is filtered to remove cesium or sodium hydrides, which may tend to form a solid residue. The material can be further purified before it is sent to a holding manifold. From here, it is pumped to the collector or to the boiler.

There is a secondary beneficial characteristic of having a metal vapor plume crossing the beamline. The plume acts as a gas curtain that prevents background hydrogen from diffusing through it. In theory and based on data from the Soviet Union, ${ }^{4-11}$ this effect can produce an order-of-magnitude pressure drop across the double-charge-exchange cel1. This pressure drop enables us to pump the large quantity of gas escaping from the source at a higher pressure, and reduces the flow of gas into the accelerator region.

A current of $\mathrm{I}^{+} \mathrm{A}$ enters the double-charge-exchange cell. When it leaves as a $20 \%$ negative beam, it has collected $1.2 \mathrm{I}^{+}$A of electrons. This electron flow must be provided for in the overall beamline circuit. However, on a local scale, it must come from the cesium vapor plume. As this electron flow takes place, a positive plasma is formed in the plume that expands in all directions until the electron debt is equalized. Work at Lawrence Berkeley Laboratory is determining the consequences of this placma formation with respect to metal vapor confinement. It may be necessary to provide a source of 
electrons (an emitter) in the cesium plume prior to the point at which it crosses the beamline. With this source of electrons, the plasma formation and therefore its growth orit of the plume will be limited.

The nozzle design and the characteristics of the plume as it undergoes an isentropic expansion are described elsewhere. ${ }^{4-12}$ The mass of cesium flowing across the beamline is determined from the relation $Q_{M V}=n i w v$, in which $n=$ density, $Q=$ length of plume along the beamline, and $v=$ velocity. For this cell with width, $w=110 \mathrm{~cm}$, the vapor flow is $1.3 \mathrm{~g} / \mathrm{s}$.

The mass flow is used to determine the power required to operate the double-charge-exchange cell. The majority of the eutectic is maintained at $-20^{\circ} \mathrm{C}$ with constant refrigeration losses due to conduction and the energy of the incoming vapor plume on the collector. Vapor in the plume must be heated from -20 to $425^{\circ} \mathrm{C}$. Also, the manifolds, slits, and nozzle surfaces must be maintained at $425^{\circ} \mathrm{C}$ to guarantee the proper flow characteristies. Circulating the eutectic involves a pump load, and the liquid nitrogen-cooled venetian-blind collector vanes add to the power required by the ce1:. An estimate for this total is $30 \mathrm{~kW}$. Neutral-beam power requirements are often expressed as electrical input power divided by injected neutra1-beam power. This double-charge-exchange cell has a power need (as defined above) of $0.023 \mathrm{We} / \mathrm{W}$.

400-kV ACCELERATION COLUMN

The 400-kV He acceleration column will be similar to the 1.2-MV Pierce-type acceleration column previously described. ${ }^{4-13}$ The potential along the beamline will vary according to the planar Child's Law; i.e., 


$$
\begin{aligned}
j & =(4 / 9) E_{0}(2 q / m)^{1 / 2} v(x)^{3 / 2} / x^{2} \\
& =5.46 \times 10^{-8} v(x)^{3 / 2} / x^{2} \text { for } H^{-},
\end{aligned}
$$

in units of $A, V$, and $\mathrm{cm}$. The current density is $\mathrm{j}=5.34 \mathrm{~A} /(7 \times 50) \mathrm{cm}^{2}$ $=0.016 \mathrm{~A} / \mathrm{cm}^{2}$; therefore, the length of the acceleration column is $30 \mathrm{~cm}$. As shown in Fig. 4-6, the acceleration column will be divided into four stages by three intermediate electrodes at positions $x=7.5,15.0$, and $22.5 \mathrm{~cm}$. The required voltages relative to the $\mathrm{H}^{+}$ion source at these points are 55,170 , and $285 \mathrm{kV}$; these voltages will be supplied by a voltage divider feeding the intermediate electrodes. The maximum electric field required is on 1 y $17 \mathrm{kV} / \mathrm{cm}$.

It is necessary to prevent the electrons in the background plasma from entering the 400-kV acceleration colum. The most straightforward technique for electron suppression is the immersion of two or more grids in the lowenergy beam, biased to repel thermal electrons produced by ionization of background gas and cesium vapor. Electron suppression by immersed grids has been tested by a series of direct conversion experiments at Lawrence Livermore Laboratory and at Fontenay-aux-Roses. ${ }^{4-14}$ Radiation-cooled …1s immersed in the low-energy beam have been proven effective for suppressing plasma electrons if the beam power density is not greater than the limitation established by thermionic emission from the negative grid wires (100 to $200 \mathrm{~W} / \mathrm{cm}^{2}$ ).

Figure 4-7 shows a grid assembly at the entrance of the 400-kV acceleration column. The assembly consists of three sets of aligned grids of $80 \%$ transparency. The suppression field is established between Grid 1 at ground potential and Grid 2 at -300 v. Fringing field effects are reduced by Grid 3 , also at $-300 \mathrm{v}$. Spacing between wires is determined by the requirement that the potential midway between wires be negative relative to the plasma potential by several times the electron temperature. The wire diameter is 


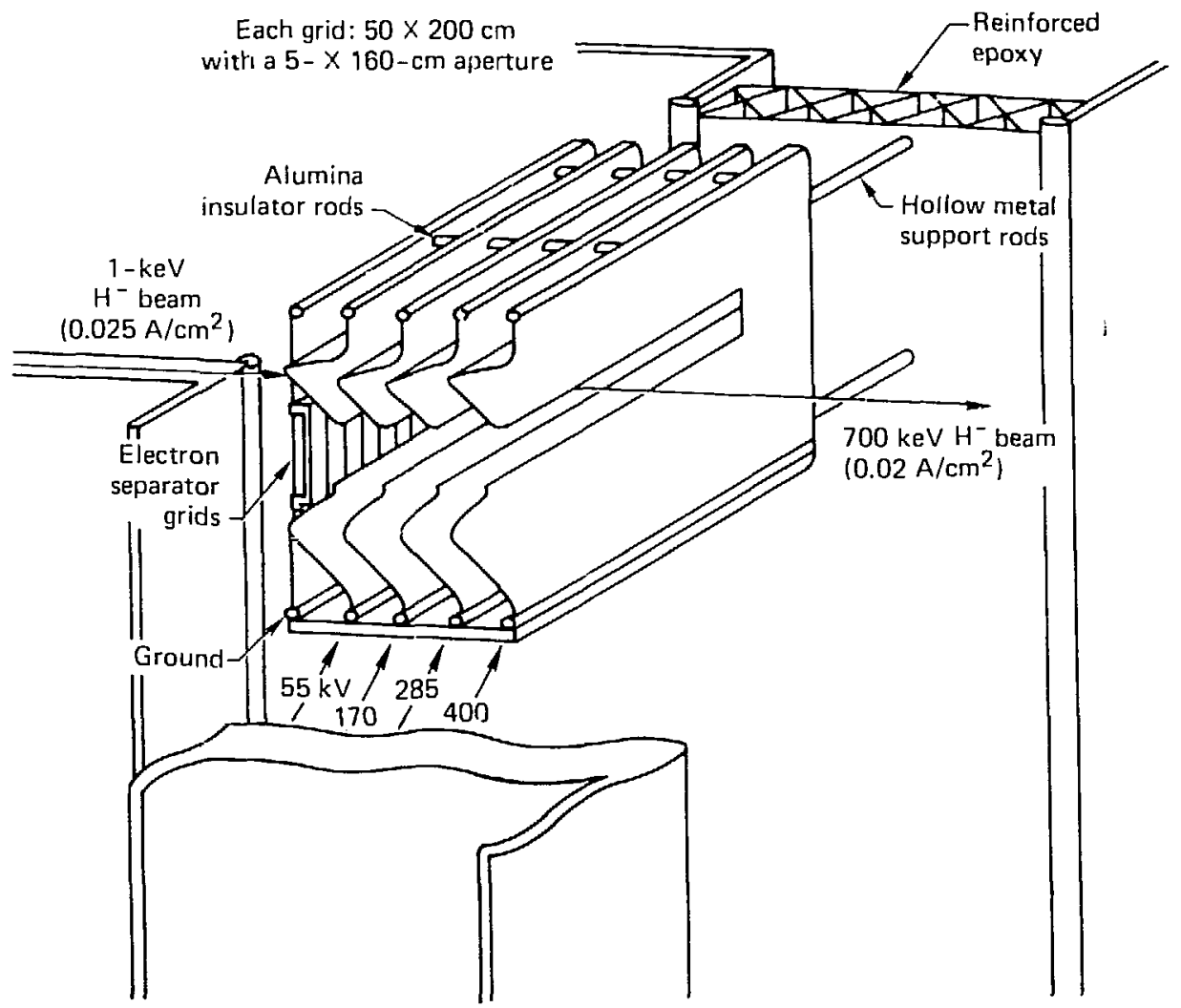

Fig. 4-6. Details of 400-keV grid assembly.

determined by the sputtering lifetime required and by the effect of wire diameter upon fringing fields.

The negative grids will collect about $1 \mathrm{~mA} / \mathrm{cm}^{2}$ of positive ions (mostly $\mathrm{H}_{2}^{+}$) extracted from the background plasma. The grid power requirement at $300 \mathrm{~V}$ will be only about $100 \mathrm{~W}$.

It is possible to ground either end of the acceleration column and to operate the other end at positive or negative high voltage. It is convenient in this design to ground the ion source end of the accelerator to simplify the 


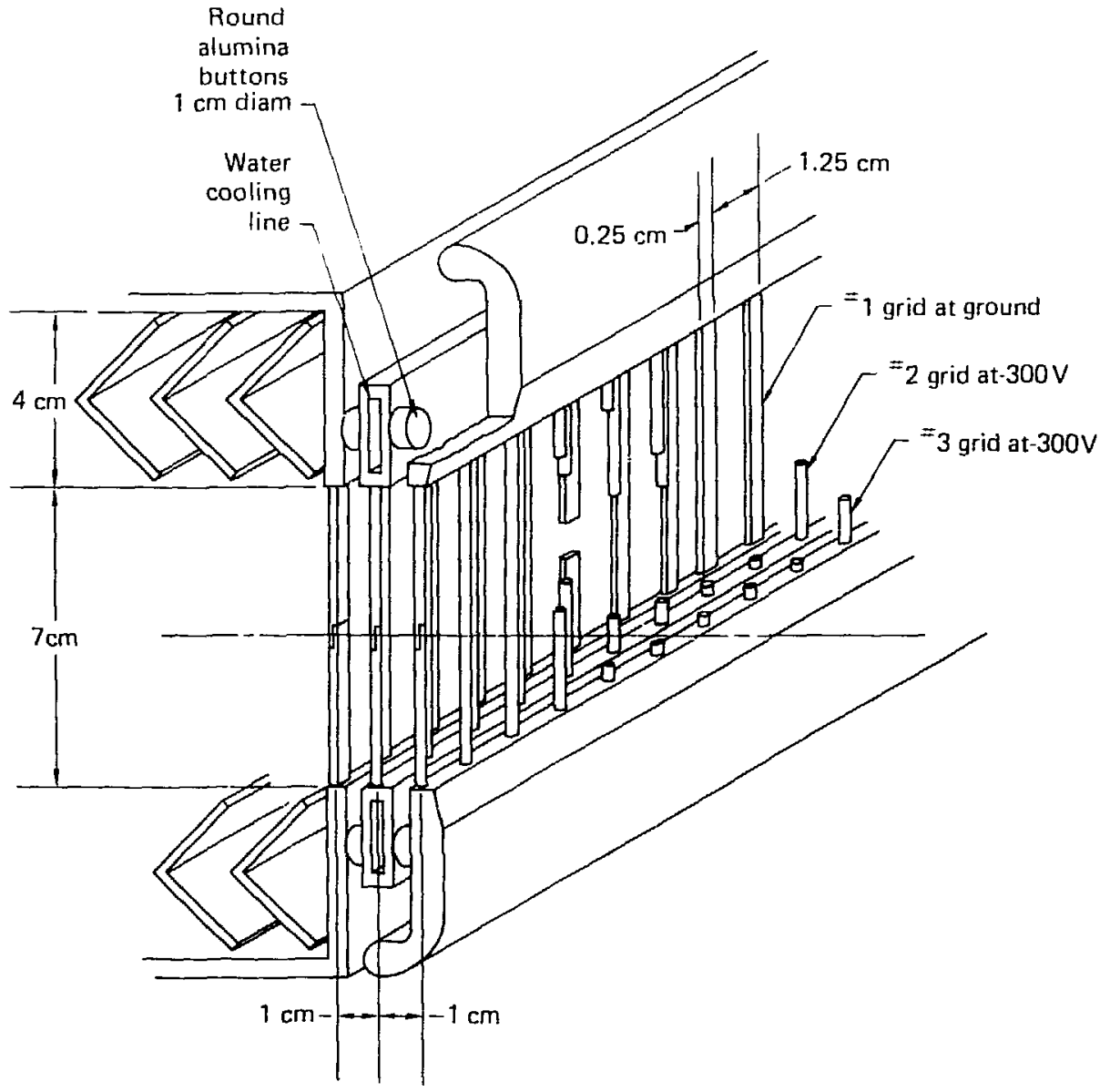

Fig. 4-7. Electron suppression grids to prevent thermal electrons from entering $\mathrm{H}^{-}$accelerator. 
pumping of gas from the ion source and to simplify the many electrical and mechanical connections to the low-energy positive ion source and to the charge-exchange cell. The output end of the accelerator will be at $+400 \mathrm{kV}$, where the $\mathrm{H}^{-}$beam will enter a region of uniform potential including tie stripping cell, the ion deflector, and the ion dump collecting the charged components emerging from the stripping cell. A transition region is then required to return the neutral beam to ground potential for injection into the TMR.

Equipotential shields are required on all sides of the region at $+400 \mathrm{keV}$ to maintain uniform potential gradients. We specify four equipotential shields at voltages corresponding to those of the intermediate electrodes along the acceleration column.

The energy released during a high-voltage breakdown $\left({ }_{-1}^{1} \mathrm{CV}^{2}\right)$ must be not more than a few joules to avoid pitting and deconditioning the electrodes. ${ }^{4-15}$ This limitation requires an external voltage protective circuit to isolate the effects of a breakdown and to suppress the arc. The limitation on capacitively stored energy also limits the areas and spacings of high-voltage electrodes and shields.

The maximum size of the acceleration column is limited by the interelectrode capacitance of the fourth acceleration stage, for which $\mathrm{V}=115 \mathrm{kV}$ and the spacing $d$ is a minimum of $7.5 \mathrm{~cm}$. Because of the electrode shape the average value of $\mathrm{d}$ will be at least $10 \mathrm{~cm}$. In the vacuum of $10^{-4}$ Torr we can tolerate an energy release of 2 to $10 \mathrm{~J}$ per breakdown. This release leads to an allowable interelectrode capacitance of $250 \mathrm{pF}$ and a maximum electrode area of $3 \mathrm{~m}^{2}$. This area is ample for the $400-\mathrm{kV}$ acceleration column. Because of the stored energy limitation, several parallel sets of equipotential shields are required to enclose the $400-\mathrm{kV}$ uniform potential region. 
Electrons and positive ions will be produced within the acceleration column by electron detachment of $\mathrm{H}^{-}$and by ionization of the background $\mathrm{H}_{2}$. Some of the electrons and positive ions will be collected by the intermediate electrodes and will constitute a load on the voltage divider. This load can be computed by integrating the rates of electron and ion production along the 30-col beam path through the accelerator.

The rate of electron production within the acceleration column by detachment from $\mathrm{H}^{-}$and by ionization of $\mathrm{H}_{2}$ gas is

$$
\int n_{\text {gas }}\left(\sigma_{-1,0}+\sigma_{i}\right) I^{-} d x=0.33 A,
$$

if $n_{\text {gas }}=3.3 \times 10^{12} \mathrm{~cm}^{-3}$ and $\mathrm{I}^{-}=5.342 \mathrm{~A}$. In the integration we have taken into account the variation of the cross sections with $\mathrm{H}^{-}$energy. Similarly, the rate of slow ion production by ionization of $\mathrm{H}_{2}$ is

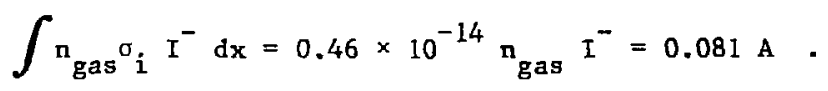

Most of the ions and electrons will be accelerated with the beam, but some fraction will be collected by the electrodes. We should design for an electron load of about $0.03 \mathrm{~A}$ and for an ion load of about $0.01 \mathrm{~A}$ collected by the intermediate electrodes and constituting a load upon the potential divider. This computation assumes that the background gas density is equivalent to a gas pressure of $10^{-4}$ Torr at room temperature.

The accelerator efficiency will be determined by the power consumed by the electrons and slow ions produced in the background gas. When we assume that the total energy consumed by an ion-electron pair or by an electron detachment is 400-keV, the power loss is $132 \mathrm{~kW}$. 
Experience is available on long-term operation of accelerators in the 400-kV voltage range at lower beam currents. 4-16́

\section{Insulation Problems}

Accelerator standoff insulators will be ceramic with a resistive coating to avoid electrostatic charge accumulation. This resistive coating must be developed for the purpose because previous attempts to apply such coatings have resulted in enhanced emission of secondary electrons. ${ }^{4-17}$ The insulators must be shielded from direct exposure to radiation and to particles emitted by the beam. Standoff insulators are also required to support the equipotential shields. Figure 1-5 shows the standoff insulators and the other insulators required.

A large portion of the main vacuum tank will be at $400 \mathrm{kV}$ to enclose the uniform potential region. This is done by enclosing the main vacuum tank in a pressurized atmosphere of sulfur hexafluoride, which has an insulation strength several times better than vacuum or atmospheric air. A11 of the external high-voltage circuitry is enclosed in the same SF 6 pressurized tank, and will also be enclosed in equipotential shields for high-voltage reliability.

Two large-area epoxy insulators are required to isolate the $400-\mathrm{kV}$ portion of the vacuum tank from the grounded portion. The epoxy insulators are exposed to vacuum on one side and to the pressurized $5 F_{6}$ on the other side. The geometry of the vacuum tank provides shielding from $x$-ray and particle bombardment .

A filled epoxy matching the coefficient of expansion of the metal housing and reinforced with fiberglass rods is cast in place to form the insulator. ${ }^{4-18}$ A conductive coating is applied to the epoxy surface to suppress secondary emission and to inhibit outgassing. 


\section{High-Voltage Protective Circuit}

The high-voltage protective circuit must be designed to extinguish a breakdown across a high-voltage gap before the breakdown becomes fully developed and without perturbing the voltages across adjacent gaps in the highvoltage assembly. The protection is accomplished by limiting to a few joules the energy released in a breakdown and thereby avoiding the production of charge carriers by damage to electrode surfaces.

The protection is easier to accomplish in high vacuum than in gas discharges because of the slower risetime $(1$ to $2 \mu \mathrm{m})$ and lower peak currents of high vacuum sparks in comparison with gas discharge sparks. Consequently, the allowable release of stored energy in a breakdown without damaging electrode surfaces by pitting is several joules perhaps as high as $40 \mathrm{~J} .{ }^{4-15}$

Safety circuits are required in addition to the arc suppression circuits. In Fig. 4-8 we show the high-voltage protective circuit, which includes seven components. We shall describe these components beginning at the acceleration electrodes and working back to the $400-k V$ power supply to show how the components perform the required function.

As shown in Fig. 4-8, each branch of the high-voltage circuit requires a voltage divider to supply the three intermediate electrodes of each acceleration column and each assembly of equipotential grids. The current capacity of the voltage dividers should be larger than the electrical load on the intermediate electrodes, about $0.03 \mathrm{~A}$. The voltage dividers may be either resistors or zener diodes and will consume about $40 \mathrm{~kW}$.

The RC time for recharging the interelectrode capacitance will be $0.7 \mu s$, representing the recovery time to reestablish normal voltage after the quenching of a breakdown. Faster operation and less power loss is possible if zener diodes are used in series with resistors. 


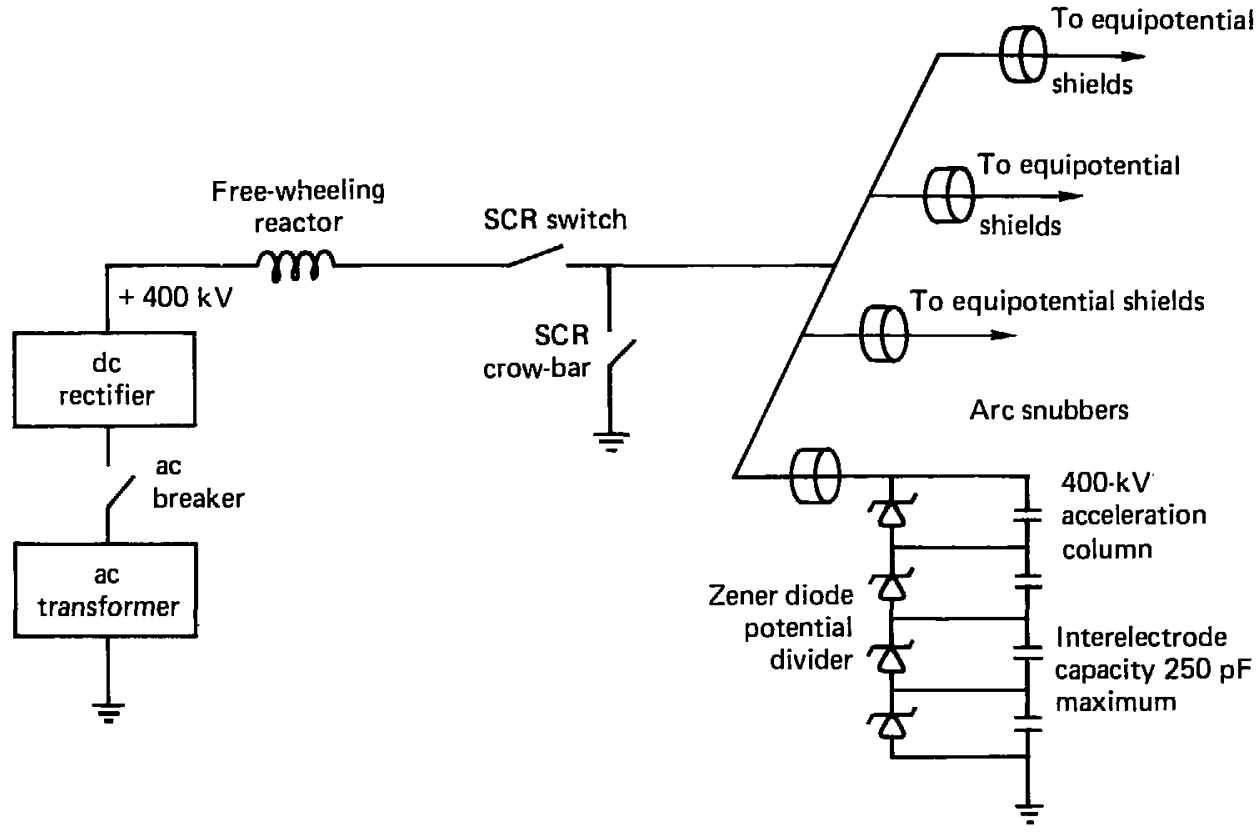

Fig. 4-8. High-voltage protective circuit.

The electrical load of the equipotential grids will be very small because $I^{-}=0$ and $n_{\text {gas }}$ is reduced by an order of magnitude. A few milliamperes of divider current will be sufficient to supply these grids, with negligible power consumption.

Each branch of the high voltage circuit feeding the acceleration column and the four or five sets of equipotential grids will be protected by an arc snubber ${ }^{4-19}$ capable of dropping the applied voltage within a microsecond of the onset of a breakdown between electrodes. The arc snubber consists of an assembly of ferromagnetic cores in which eddy currents are generated by the fast current pulse caused by the breakdown. 
The eddy currents in the arc snubber have the effect of a time-dependent pure resistance. Therefore, the total voltage applied to the acceleration column will be reduced at the onset of an arc such that the arc will be quenched at one electrode gap without increasing the voltages at adjacent gaps. With proper design most of the arcs will be quenched by the snubber and voltage divider, which lave no active components.

Tests of snubber performance for a single-gap 40-kV accelerator indicate time constants between 0.07 and $0.27 \mu s$, depending upon the capacitance and initial voltage. This range is fast enough to quench a vacuum arc with a risetime of several microseconds.

Other high-voltage elements, such as the electrostatic shields that do not carry significant currents, can be protected by inductive type arc suppressors ${ }^{4-20}$ which are considerably smaller components than the magneticcore arc snubbers previously described.

It will be necessary to back up the passive arc snubber with an actively operated crowbar and high-voltage switch to interrupt the high-voltage de power in about $2 \mu \mathrm{s}$. A wel1-tested and economical design for this purpose consists of a string of SCR's as a switch in series with the dc high-voltage power supply and load, and a second string of SCR's as a crowbar to ground. It is possible to open the SCR series switch by momentarily shorting the power supply to ground through the crowbar. The crowbar is then reopened by an LC commutation network.

An SCR valve operating on this principle has been developed and tested at $400-\mathrm{kV}$ for HVDC transmission. ${ }^{4-21}$ A similar valve is now operating at the 120-kV LBL test stand. 4-22

Vacuum tubes have also been used as high-voltage switches and crowbars, but not at $400 \mathrm{keV}$. It appears that SCR's are less expensive and require less development for this purpose than vacuum tubes. 
The system will be programmed to close the SCR switch and to reestablish operation a few milliseconds after an interruption. The interruption time should be short in comparison with the $0.1-s$ ion lifetime in the TMR plug. ${ }^{4-23}$ If for some reason this is not possible within a specified time interval, it may be necessary to open the ac circuit breaker.

It will be necessary to protect the 400-keV power supply from overcurtent and over-voltage when the SCR crowbar is fired. The current limitation must be effective until either the SCR crowbar is reopened by the commutation circuit or until the ac breaker is opened, which may be a maximum of $1 / 60$ s later when the ac current passes through zero.

The commutation circuit in the 120-kV LBL system reopens the SCR crowbar in a ha1f-period of $23 \mu \mathrm{s}$. Therefore, a current-limiting inductance of 0.1 Henry is sufficient to limit the current rise to $100 \mathrm{~A}$ while $400 \mathrm{kV}$ is applied to the inductance for $23 \mu \mathrm{s}$. Such a pulsed voltage can be supplied by a free-wheeling back-biased reactor.

The ac circuit breaker would normally be installed to interrupt the low-voltage ac feeding the primary of the transformer in the 400-kV power supply. However, it may be considered more positive to install the breaker in the high-voltage output of the transformer, as shown in Fig. 4-8. A highvoltage ac breaker interrupts the circuit when the current goes through zero, so the maximum delay time may be $1 / 60 \mathrm{~s}$.

There are several motivations for changing the ac frequency to $400 \mathrm{~Hz}$ rather than $60 \mathrm{~Hz}$. The faster response time of the breaker is one of these motivations. Other motivations would be the sma1ler size of the transformer core and of the ripple filter, if any. A cost study is required to resolve this possibility. 
A great saving in capital cost and operating efficiency is possible if the 40-MW high-voltage power supply is not regulated in the usual sense of the word. A large shunt regulator or series regulator would be very expensive and would consume a large fraction of the output power. If the TMR can tolerate 5 to $10 \%$ fluctuations of injected neutral-beam energy, a large savings would result.

It would be necessary to regulate the arc power of the ion sources to follow fluctuations in power supply voltage in order to maintain the proper conditions for optimized beam optics. Arc power regulation is much more simple than high-voltage acceleration regulation because of the low voltages and low power involved. ${ }^{4-15}$

It is also possible to eliminate the need for regulation by making the extracted current densicy and thereby the beam current fluctuate with the $3 / 2$ power of the high voltage. This fluctuation results in a beam of constant cross section and could be accomplished by making the positive ion extraction voltage track with the high-voltage ripples.

METAL VAPOR STRIPPING CELL

The physics of atomic collisions in a metal vapor stripping cell have been described in our irevious reactor designs. 4-13 The mechanical design of the metal vapor stripping cell is essentially the same as that of the cesium vapor double-charge-exchange cell previously described. Therefore, the only new material required in this section is a discussion of some design decisions applying to this application. 
The purpose of the stripping cell is to convert the $\mathrm{H}^{-}$beam to $\mathrm{H}^{0}$ at maximum efficiency by collisional electron detachment. The maximum conversion efficiency $\mathrm{F}^{0}$ is limited by ionizing collisions converting the $\mathrm{H}^{0}$ to $\mathrm{H}^{+}$. The conversion efficiency is relatively high (up to $65 \%$ for vapor cells or $85 \%$ for plasma cel1s) because the cross section for detachment of the first electron is several times larger than the ionization cross section. For 400-keV H', the optimum stripping cel1 thickness for maximum $F^{0}$ is $1 \times 10^{16}$ vapor atoms $/ \mathrm{cm}^{2}$ or $0.2 \times 10^{16}$ plasma ions $/ \mathrm{cm}^{2}$. Such a plasma cell without background gas is not a trivial development; therefore, we choose a vapor cell for this application that is adequate for the purpose.

Metal vapors are preferred to other types of gases because they can be easily condensed without large gas pumping systems. Other condensable vapors such as $\mathrm{CO}_{2}$ or $\mathrm{H}_{2} \mathrm{O}$ have disadvantages, such as electrostatic charge buildup on condensed insulating surfaces and production of incondensable gases by dissociation of molecular vapors. Atomic gases such as argon, xenon, etc., are undesirable because they form thermally insulating layers on the condensing target and therefore can be used for a limited time only. The choice of metal vapor for the stripping cell will be determined largely by the scattering of the vapor by the beam. This scattering will be the only major loss of vapor, if we use LN cooled baffles, suitably located, and a plug type nozzle. A low-z metal such as lithium may be less objectionable as a reactor contaminant than a high-z metal, but it is more subject to loss by momentum transfer from the $\mathrm{D}^{-}$beam to the target.

For several reasons it will be necessary to develop the metal vapor stripping cells to such a level of efficiency that there is negligible vapor contamination of the system outside the cells. It is assured that a metal vapor flux of less than $10^{11}$ atoms $/ \mathrm{cm}^{2}-\mathrm{s}$ will be tolerable. This flux should be sputtered away by a background current density of several $\mu \mathrm{A} / \mathrm{cm}^{2}$. 
Cold fingers in noncritical locations will be required to collect whatever does escape. With these assumptions any of the alkali metals will be satisfactory for the stripping cell.

Finally, a design decision must be made whether or not to operate the stripping cell in a mode suitable for beam direct conversion, in which the kinetic energy of the residual charged beam is electrostatically recovered to improve the overall efficiency. As previous $1 y$ described, ${ }^{4-13}$ beam direct conversion probably requires an overdense stripping cell to eliminate the residual $\mathrm{H}^{-}$. Consequently, the conversion efficiency $\mathrm{F}^{0}$ will not be optimum. The $\mathrm{F}^{0}$ penalty for an overdense plasma cell is small, but is somewhat more significant for an overdense vapor stripping cel1. For an optimized vapor cell $\mathrm{F}^{0}=0.6$, while for an overdense cell $\mathrm{F}^{0}$ may be as small as 0.45 .

Furthermore, we believe that thermal coniersion of the residual charged beam energy is practical only if electrostatic direct conversion is not used. The two types of beam energy conversion may not be compatible.

From these considerations we can convute the injection efficiency of three alternative systems using a consistent set of assumptions:

$$
\begin{aligned}
& \mathrm{F}^{0}=0.6 \text { for optimized ce11; } 0.45 \text { for overdense cel1 } \\
& \text { Cost of } \mathrm{H}^{-} \text {production }=20 \mathrm{keV} / \mathrm{H}^{-}
\end{aligned}
$$$$
\text { Thermal conversion efficiency }=30 \%
$$$$
\text { Direct conversion efficiency }=7 \mathrm{C}^{\prime \prime},
$$

Under these assumptions the injection efficiencies for three alternative systems are as follows, not including thermal conversion in the $\mathrm{H}^{0}$ beam dump:

System

1.

2.

Optimized stripping cell without thermal conversion Optimized :tripping cell with thermal conversion in the $\mathrm{H}^{+}$and $\mathrm{H}^{-}$collectors

3. Overdense stripping cell with beam direct conversion of $\mathrm{H}^{+}$on $1 \mathrm{y}$
Percent

57.1

64.5

67.7 
The difference in efficiency between system 2 and system 3 is trivial, depending upon details in the assumptions. System 2, with thermal conversion, has two advantages over System 3:

- The beam direct-converter system, operating upon $\mathrm{H}^{+}$, requires a collection electrode at a potential $400-\mathrm{kV}$ above the stripping cell potential; this makes a total voltage difference of $800 \mathrm{kV}$ relative to the negative ion source, while the thermal convezter requires a voltage difference of only $400 \mathrm{kV}$.

- The useful beam per unit area is greater with an optimized stripping cell.

For these reasons we choose an optimized stripping cell with thermal conversion of the residual $\mathrm{H}^{-}$and $\mathrm{H}^{+}$beams, these beams will be approximately equal in current as they emerge from the stripping cell.

DISPOSAL OF CHARGED BEAMS

The charged beam emerging from the optimized stripping cell will consist of roughly equal currents of $\mathrm{H}^{+}$and $\mathrm{H}^{-}$that must be deflected from the neutra1-beam line and disposed of. Magnetic deflection using the fringing field of the magnetic containment device is the usual solution for this. However, the TMR fringing field at this point is in the wrong directon for charged beam deflection. A sweep magnet suitable for a beam cross section $7 \times 50 \mathrm{~cm}$ would occupy an inconveniently large volume near the reactor. Therefore, it is expedient to use a transverse electrostatic field of about $5 \mathrm{kV} / \mathrm{cm}$ to deflect the $\mathrm{H}^{-}$and $\mathrm{H}^{+} \pm 15^{\circ}$, as shown in Figs. 1-5 and 4-7. The deflection electrodes should be about $50 \mathrm{~cm}$ long, followed by a drift space of $75 \mathrm{~cm}$ to deflect the charged beams $\pm 20 \mathrm{~cm}$. The beams will then be collected at grazing incidence by pressurized water-cooled collectors 
connected to a steam generator for thermal energy recovery. No attempt will be made to decelerate the beams before collection, since this may be difficult when both positive and negative ions are involved in the same system.

The electric field induced by separation of the $\mathrm{H}^{-}$and $\mathrm{H}^{+}$beams will probably be neutralized by the background plasma and therefore will not affect the trajectories. However, it is easy to compute an upper limit for the electric field strength using a one-dimensional model and Gauss's 1 aw, neglecting the neutralization by background plasma. This upper limit is $E=j b / E_{0} \dot{v}$, where $\mathrm{jb}=2.2-\mathrm{A} / \mathrm{m}$ charged-beam current per unit length. Therefore, $E$ is not more than $0.7 \mathrm{kV} / \mathrm{cm}$, which is small compared to the applied field of $5 \mathrm{kV} / \mathrm{m}$. The maximum possible deflection due to bean space charge is $5 \mathrm{ctu}$, which can be allowed for in the design of the collectors.

Stripping of the $\mathrm{H}^{0}$ and $\mathrm{H}^{-}$in this region will be small because we are designing for a residual gas pressure of $10^{-5}$ Torr. The largest gas load in this region will be from the charged beam dumps.

In order to avoid loading the deflection electrodes with electrons produced in the stripping cell, we will operate one electrode at $+400 \mathrm{kV}$ (the same potential as the stripping cell) and operate the other electrode at $+340 \mathrm{kV}$. Consequent $1 \mathrm{y}$, the $\mathrm{H}^{-}$will be accelerated by a few percent while passing through the deflection electrodes and the $\mathrm{H}^{+}$will be decelerated. The asynmetry in the deflections will be small and not important.

VACUUM PUMPING AND DRIFT DUCT BLOCKING

In any negative-ion-based neutral-beam system, a tremendous volume of gas must be pumped relative to the neutral beam current.

The positive-ion source has a gas efficiency of $30 \%$. Thus, for every ampere of positives leaving the source, $2.33 \mathrm{~A}$ of equivalent neutral current escapes. 
The double-charge-exchange ce 11 is $20 \%$ efficient; i.e., for every ampere of positives that enters the cel1, $0.2 \mathrm{~A}$ is changed to negatives but $0.8 \mathrm{~A}$ escapes as neutrals that must be pumped away.

An optimum cesium stripping cell is $62 \%$ effective. Then for every ampere of high-energy neutral beam that leaves the cell, $0.61 \mathrm{~A}$ of high-energy residual ions are lost to the beam dumps. Gas in this region must be pumped at the lowest possible pressure as any reionization of the beam on background gas is a direct loss of high-energy beam.

The advantage, as far as pumping goes, of negative-ion-based systems using double-charge-exchange is that the additional cesium vapor plume that crosses the beamline acts as a gas curtain. This high resistance to gas flow through the cell allows us to pump more of the gas in a higher pressure zone ahead of the double-charge-exchange cell. The metal vapor stripping cell also prevents gas in the accelerator region from diffusing downstream into the drift duct.

Figure 4-9 is a graphic representation of gas flow in the plug injector, and illustrates that the negative-ion gas efficiency of a double-chargeexchange source is $4 \%$, i.e., $4 \%$ of input gas is converted to negative ion beam. Figure 4-9 also shows that for an ampere of neutral-beam injected, 39 equivalent $A$ must be pumped at various pressures.

The largest fraction of gas is pumped at $2 \times 10^{-3}$ Torr in the region between the source and the double-charge-exchange cell. From Table 4-1 and Fig. 4-9, the gas involved is

$$
Q_{A}=\frac{0.19 \times \frac{70 \%}{2.5 \%} \times 3.2 \mathrm{~A}}{2 \text { ions per molecule }}=8.5 \frac{\text { Torr-litre }}{\mathrm{s}}
$$

At $2 \times 10^{-3}$ Torr, this throughput requires a pumping speed of $4250 \mathrm{litres} / \mathrm{s}$. This speed will be accomplished using two large diffusion-ejector pumps . 


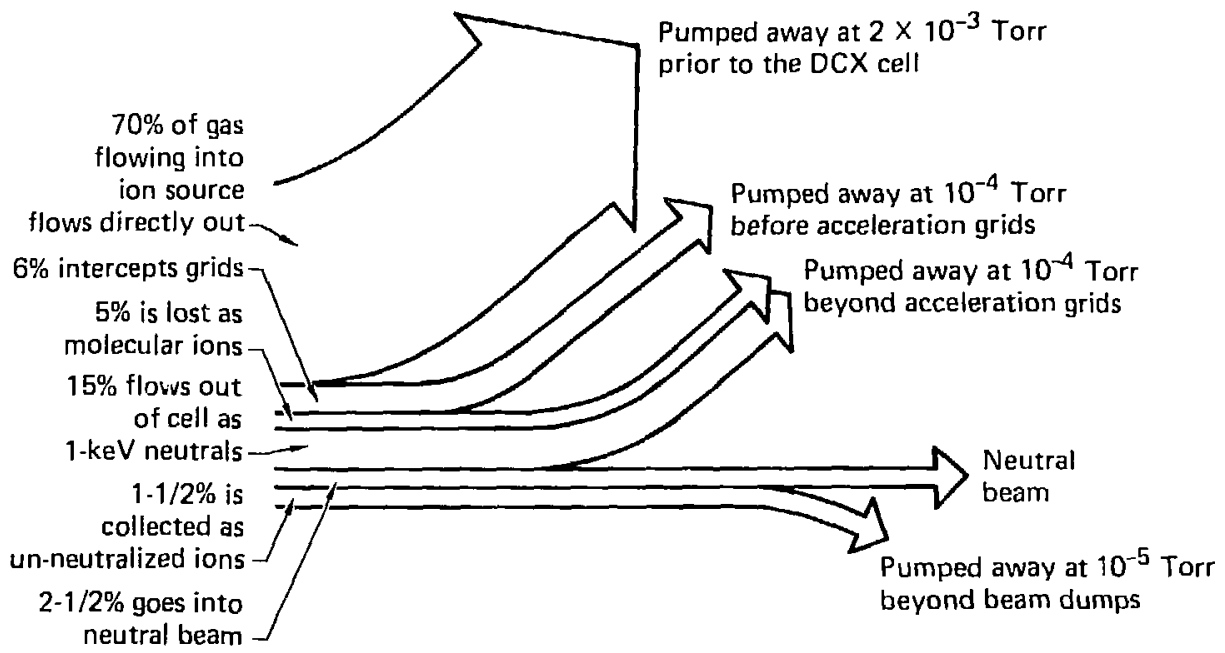

Fig. 4-9. Beamline pumping load-97.5\% of input gas.

The throughput required in the region between the double-charge-exchange cell and the stripping cell is

$$
Q_{B}=\frac{0.19 \times 26 \% / 2.5 \% \times 3.2 \mathrm{~A}}{2 \text { jons per molecule }}=3.2 \frac{\text { Torr-1itre }}{\mathrm{s}} \text {. }
$$

This throughput will be accomplished using recycling cryocondensing panels, shown in Figure 4-10, in the region surrounding the acceleration grids. The area of cryopanel in this region is

$$
A_{B}=\frac{3.2 \text { Torr-1itres } / \mathrm{s}}{10^{-4} \text { Torr } \times 4 \text { litres } / \mathrm{s} / \mathrm{cm}^{2}}=0.8 \mathrm{~m}^{2} \text {. }
$$

The third pumping region is between the stripping cell and the reactor and involves pumping residual gas from the beam dumps at $10^{-5}$ Torr. Because the vapor pressure of $\mathrm{H}_{2}$ at $4.2 \mathrm{~K}$ is greater than $10^{-7}$ Torr, the pane 1 


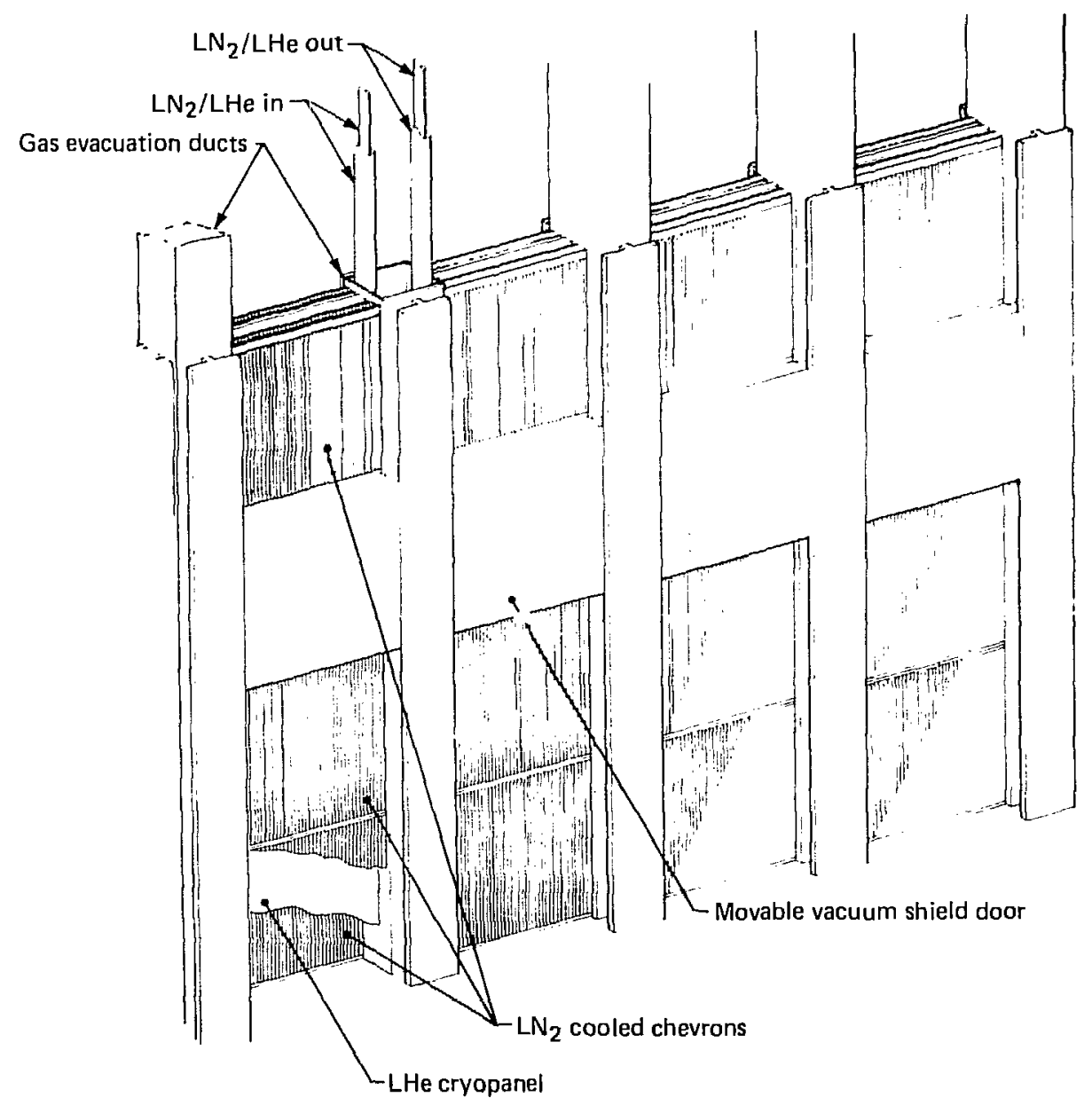

Fig. 4-10. Cryopanels for continuous operation. 
must be subcooled to $3.8 \mathrm{~K}$ in order to maintain a sticking fraction of nearly unity. The panel area required is

$$
A_{C}=\frac{\frac{1}{2} \times 0.19 \times 1.5 \% / 2.5 \% \times 3.2 \mathrm{~A}}{10^{-5} \text { Tort } \times 4 \text { litres } / \mathrm{s} / \mathrm{cm}^{2}}=0.5 \mathrm{~m}^{2}
$$

Modern experiments using neutral beams in a pulsed application have encountered beam attenuation in the drift duct leading to the reactor. ${ }^{4-24}$ This beam attenuation is primarily due to out-gassing of the duct walls caused by bombardment with reionized high-energy particles. Drift duct blocking will not present a problem in this design for two reasons. First, the neutral-beam current density is low enough that the density of particles reionized and striking the walls will be reduced. Second, and more important, modern neutralizers use gas while the TMR design uses metal vapor; i.e., where the TMR stripper isolates the duct from the majority of beamline gas, the modern neutralizers are suppliers of the gas that initiates the duct blocking phenomenon.

PROTECTION AGAINST NEUTRON, MICROWAVE, AND SYNCHROTRON RADIATION

An injector can be damaged by excessive levels of radiation impinging on sensitive elements. The usual form of this sensitivity is degradation due to bulk heating. The TMR plug injector will be subjected to four main types of radiation (neutrons, gamma, synchrotron, and microwaves injected for If heating) which it must survive for one year of dc operation, at least. Actual Monte Carlo calculations of the neutron and gamma radiation striking various components have not yet been made for this injector design. This discussion will be based on estimates of synchrotron and microwave radiation as well as comparisons to Monte Carlo calculations that have been performed for other injector designs. 
D-D reactions produce $2.45-\mathrm{MeV}$ neutrons, and $\mathrm{D}-\mathrm{T}$ reactions produce 14.1-MeV neutrons. Neutrons produced in the plug plasma will be able to enter the neutral beam duct at various angles, with some small fraction streaming directly down the beamline until stopped either by a beamline component or the back wall. Primary neutrons striking the duct walls will produce ganma radiation and secondary neutrons. Neutron radiation from the plasma is lineof-sight dependent, and sensitive beamline components are therefore located where a direct view of the plasma is not possible. However, gammas and secondary neutrons evolved from primary neutron interactions prevent radiation levels from dropping off to zero anywhere in the injector.

Synchrotron radiation is higher in the plug than elsewhere in the reactor due to the combination of high electron temperature and high magnetic field. The synchrotron radiation and any ECRH microwave energy not absorbed by the plasma is able to turn corners based on what waveguide characteristics are perceived by the wave as it travels throughout the injector chamber.

Susceptible materials in the injector include the large-area epoxy insulating walls, the alumina insulators, and high-voltage feedthroughs, liquidnitrogen-cooled chevrons, and liquid-helium-cooled aluminum cryopanels (see Table 4-2). Each of these has been observed in Monte Carlo analysis of other beamlines ${ }^{4-25}$ and found to have sufficient radiation resistance to operate for a full year. All epoxy and LHe surfaces can be shielded from line-ofsight to the plasma to avoid most high-energy neutron and synchrotron radiation. Even so, it may be necessary to cover the inside surface of the epoxy wall with ceramic tiles to inhibit outgassing. Alumina insulators must be located in the acceleration and deceleration regions of the injector at 1ocations near the injector centerline. Previous data have shown, however, that the radiation levels at this distance from the plasma are low enough. 
TABLE 4-2. Preliminary design limits for radiation-sensitive components.

\begin{tabular}{|c|c|}
\hline Cryopane1 & $\begin{array}{l}\text { neutron and ganma heating rate design limits } \\
\text { Peak, W/kg } \\
\text { Average, } W / \mathrm{kg}\end{array}$ \\
\hline Insulator & $\begin{array}{l}\text { gamma plug neutron dose rate design limits } \\
\text { Epoxy, rad/s } \quad 5000 \\
\text { Alumina, rad/s } 1000\end{array}$ \\
\hline Insilator & 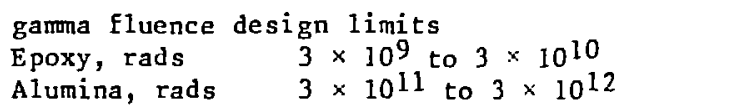 \\
\hline $\begin{array}{l}\text { Insulator } \\
\text { (Damage c } \\
\text { approxima }\end{array}$ & $\begin{array}{l}\text { - Fast neutron fluence design limits } \\
\text { Epoxy, } \mathrm{n} / \mathrm{cm}^{2} \quad 10^{18} \text { to } 10^{19} \\
\text { Alumina, } \mathrm{n} / \mathrm{cm}^{2} \quad 10^{20} \text { to } 10^{21} \\
\text { aused by a neutron fluence of } 4 \times 10^{8} \mathrm{n} / \mathrm{cm}^{2} \text { is } \\
\text { tely equal to danage caused by } 1 \mathrm{rad} \text { of gamma.) }\end{array}$ \\
\hline
\end{tabular}

Some regions of the beam duct near the first-wall aperture will require water cooling but the power density falls off rapidly due to the glancing angle of the neutrons striking the duct walls.

Two factors help to reduce the neutron radiation entering the injector. First, the plasma is lower density than in the cases studied in Ref. 4-25. Second, this is a hydrogen injector and produces a predominantly hydrogen plug plasma. Thus, the number of fusion reactions delivering neutrons to the injector will be lower than the cases for which we have Monte Carlo data that were $\mathrm{D}^{0}$ injectors. In addition, the injection of ECRH (39 MW) into each transition region of the plasma takes place $7 \mathrm{~m}$ from the injector aperture; therefore any unabsorbed ECRH is mostly attenuated before reaching the injector. A conservative estimate of ECRH power levels entering the duct is $3 \mathrm{~W} / \mathrm{cm}^{2}$.

The power density of synchrotron radiation is an order-of-magnitude higher than the ECRH level. Synchrotron radiation in the amount of 3 MW is produced in each plug. Let us assume a first-wall radius of $2.25 \mathrm{~m}$ and a line 
source of radiation $50 \mathrm{~cm}$ lang (equal to the length of the injector aperture). This assumption gives a power density of $30 \mathrm{~W} / \mathrm{cm}^{2}$ at the first wall. This level is still an order of magnitude or more below the neutron and gamma levels discussed in Ref. 4-25 so that our situation is less severe. Neutron and gamma radiation remain the most significant source of potential damage in the plug injector. We are confident that careful placement of the most sensitive elements of the beamline will enable the injector to operate reliably for a year before some maintenance may be required. 
REFERENCES

4-1. R. W. Moir, W. L. Barr, G. A. Carlson, W. L. Dexter, J. N. Doggett, J. H. Fink, G. W. Hamilton, J. D. Lee, B. G. Logan, W. 5. Neef, Jr., M. A. Peterson, and M. E. Rensink, Preliminary Design Study of the Tandem Mirror Reactor (TMR), Lawrence Livermore Laboratory, Livermore, CA, UCRL-52302 (1977).

4-2. Value Engineering: Approach to Optimizing Neutral Heating Cost and Performance, Office of Fusion Energy, U.S. Department of Energy report, DOE/ET-0113, (1979).

4-3. H. S. Staten, "Neutral Beam Injectors for the Magnetic Fusion Energy Program: Progress and Prognosis for the Future," IEEE Trans. Nucl. Sci., NS-26, No. 1 (1979).

4-4. J. H. Fink, The Design of a Continuous ly Operated 1-keV Deuterium-Ion Extractor, Lawrence Livermore Laborato:y, Livermore, CA, UCID-17825 (1978).

4-5. E. B. Hooper, Jr. and P. Poulsen, Conference Abstracts of the IEEE International Conference on Plasma Science, June 4-6, 1979, Montreal, Canada, (IEEE Service Center, Piscataway, NJ, 1979), Abstract IS4, P. 32.

4-6. N. N. Semashko, V. V. Kusnetsov, and A. I. Krylov, Proceedings of the Symposium on the production and Neutralization of Negative Hydrogen Ions and Beams, Brookhaven National Laboratory, Upton, Long Island, NY, (1970), BNL-50727, p. 170 .

4-7. K. Berman, "The Plug Nozzle: A New Approach to Engine Design," Astronautics 5, No. 4, 22, (1960). 
4-8. A. S. Schlachter, K. R. Stalder, and J. W. Stearns, "D- For tion by Charge-Transfer Collisions of 0.3 to $10-\mathrm{keV}$ Deuterium Ions and Atoms in Cesium, Rubidium, and Sodium Vapors," in Proc. of XI International Conference on the Physics of Electronic and Atomic Collisions, Kyoto, Japan (1979), (Society for Atomic Collision Research, Japan, 1979), p. 526.

4-9. J. E. Osher, Formation of a $D^{-}$Beam for Efficient Production of $D^{0}$ at High Energies, Lawrence Livermore Laboratory, Livermore, CA, UCID-15893 (1971).

4-10. M. Hansen, Constitution of Binary Alloys (McGraw-Hil1 Book Co., New York, 1958), p. 577.

4-11. W. R. EIlis, "Description of OGRA-4 at Kurchatov," Foreign Travel Report, August 28-September 30, 1978, U.S. Department of Energy, office of Fusion Energy, Germantown, MD.

4-12. J. H. Fink and J. L. Erickson, Direct Extraction of Negative Deuterium Ions to Form a $0.2 \mathrm{MeV}$ Neutral Beam, Lawrence Livermore Laboratory, Livermore, CA, UCRL-52843 (1979).

4-13. J. H. Fink and G. W. Hamilton, "A Neutral-Beam Injector for the Tandem-Mirror Fusion Reactor Delivering 147-MW of $1.2-\mathrm{MeV} \mathrm{D}^{0}$, IEEE Trans. P1asma Sci., PS-6, 417 (1978).

4-14. W. L. Barr and R. W. Moir, A Review of Direct Energy Conversion for Fusion Reactors, Lawrence Livermore Laboratory, Livermore, CA, UCRL-78204 (1976), in Technology of Controlled Fusion, Second ANS Topical Meeting, Richland, WA (1976).

4-15. W. R. Baker and D. B. Hopkins, Present and Future Technology of High Voltage Systems for Neutral Beam Injectors, Lawrence Berkeley Laboratory, Berkeley, CA, LBL-7261 (1978), presented at Plasma Heating Development Requirements Workshop, Gaithersburg, MD. 
4-16. J. Huguenin, and G. Visconti, "CERN-PS 540-keV High Gradient Preinjector Tube: Results of Two Years" Operation and Complementary Tests," Proc. of the 3rd Intern. Symposium on Discharges and Electrical Insulation in Vacuum, Paris (1968).

4-17. D. K. Davies, "Secondary Electron Characteristics of Insulator Surfaces Before and After Impulse Flashover," 8th Intern. Symposium on Discharges and Electrical Insultation in Vacuum, Albuquerque, NM (1978), (Sandia Laboratories, Albuquerque, NM, 1978).

4-18. J. P. Burkhart and C. F. Hofmann, "Applications of Cast Epoxy Resins in Power Circuit Breakers" C 74 064-2, Proc. of IEEE Power Engineering Society Winter Meeting, New York, NY (1974), (IEEE, Piscataway, NJ, 1974).

4-19. J. H. Fink, W. R. Baker, and H. M. Owren, Analysis and Applisation of a Transformer Core that Acts as an Arc Snubber, Lawrence Livermore Laboratory, Livermore, CA, UCRL-79016, Rev, 2 (1979).

4-20. W. F. Praeg, "Protection of Neutral Beam Accelerator Electrodes from Sparking," in Proc. 7th Symposium on Engineering Problems of Fusion Research, Knoxville, Tenn. (1977), (IEEE, Piscataway, NJ, 1977).

4-21. C. Beriger, I. Boban, and W. Faust, "A New SCR Valve for the Performance Test Circuit of the 400-kV Working Group at Mannheim-Rheinau", Brown Boveri Review 60, No. 2/3, 100 (1973).

4-22. J. V. Franck, A. A. Arthur, L. A. Brusse, and W. Low, "An SCR Series Switch and Impulse Crowbar at the Lawrence Berkeley Laboratory for CTR Neutral Beam Development", Proc. 7th Symposium on Engineering Problems of Fusion Research, ORNL (1977), (Oak Ridge National Laboratory, Oak Ridge, TN, 1977). 
4-23. R. S. Devoto and D. J. Bender, "Plasma Mode1s for Standard Mirror Reactors," Lawrence Livermore Laboratory, Livermore, CA, UCRL-81792 (1978).

4-24. L. D. Stewart, Neutral Beam System Reionization Losses, Princeton Plasma Physics Laboratory, Princeton, NJ, PPPL-1469 (1978).

4-25. J. D. Lee, R. H. Condit, C. L. Hoenig, T. P. Wilcox, and J. L. Erickson, Progress Report on the Neutral Beam Radiation Hardening Study, Lawrence Livermore Laboratory, Livermore, CA, UCID-18017 (1978). 


\section{CHAPTER 5. BARRIER PUMP NEUTRAL BEAMS}

G. W. Hamilton, B. G. Logan

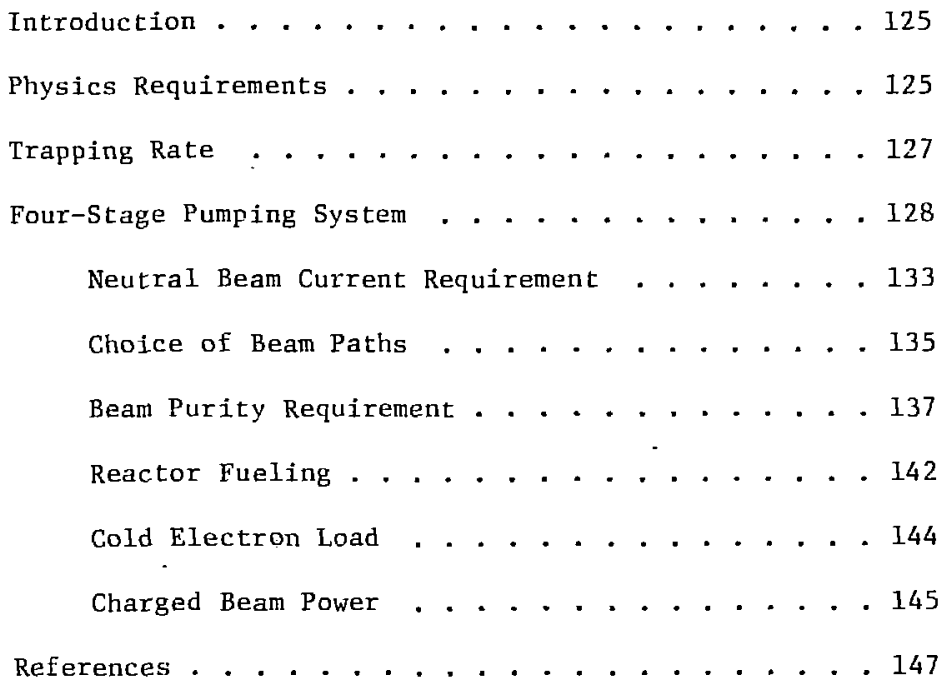




\section{INTRODUCTION}

The required low plasma density in the thermal barriers of the Tandem Mirror Reactor (TMR) ( $g_{b}$ not much larger than unity) can be maintained by pumping out trapped ions by means of charge exchange with neutral atoms ( $D^{0}$ and $\mathrm{T}^{\mathrm{O}}$ ) injected for the purpose. Conditions must be arranged so that after the charge exchange the ion trapped in the barrier goes to the wall as a neutral and the ion derived from the injected neutral goes into the central cell to become a passing particle trapped between the ambipolar plug potentials. In effect, the injected neutral has pumped out the trapped ion.

In this chapter, we deal with the special problems involved in chargeexchange pumping; we should avoid discussing neutral-beam technology that has been developed for other purposes.

\section{PHYSICS REQUIREMENTS}

For the injected neutral to escape from the barrier into the central cell the pitch angle $\theta$ after charge exchange must be sufficiently small, and the injected beam energy $E_{B}$ must be surficiently large that

$$
\sin ^{2} \theta<\left(1-\Delta \phi / E_{B}\right) / R .
$$

The potential difference $\Delta \phi(z)$ and the mirror ratio $R(z)$ are defined relative $\rightarrow$.

to thie potential $\phi_{\mathrm{e}}$ and magnetic field $\mathrm{B}_{\mathrm{mb}}=12 \mathrm{~T}$ at the entrance of the central cel1; i.e.,

$$
\Delta \phi(z)=\phi_{e}-\phi^{*}(z),
$$

and

$$
R(z)=B_{m b} / B(z) \text {. }
$$


The potential $\phi^{*}(z)$ and magnetic field $B(z)$ are defined at the point the charge exchange occurs. Obviously, the beams for the charge-exchange pump must be injected where the values of $\phi^{* *}, B$, and $\theta$ are appropriate for the beam energy used. Also, the neutral atom produced by charge exchange must escape Erom the plasma to the wall without being re-ionized by collisions with plasma ions and electrons. This escape probability should be not much less than unity.

Figure 5-1 shows those regions of velocity space in the thermal barrier where the ions either are passing through the thermal barrier or are trapped within the barrier. The equation of the hyperbola separating the trapped region Erom the passing region is the following:

$$
v_{\|}^{2}-(R-1) v_{\perp}^{2}=\Delta \phi(2 q / m)
$$

and the loss-cone angle $\theta_{\text {LC }}$ defined by the asymptotes of the hyperbcla is

$$
\theta_{L C}=\sin ^{-1}\left(R^{-1 / 2}\right)
$$

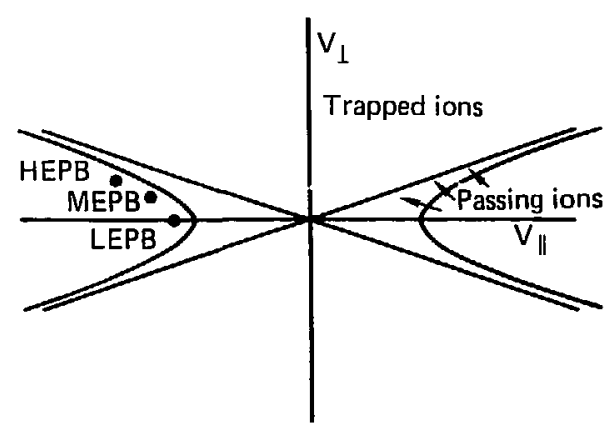

Fig. 5-1. Velocity space diagram showing the regions of velocity space at the center of the thermal barrier in which ions are either passing through the well or trapped within the wel1-asymptote angle is $\sin ^{-1}\left(R^{-1 / 2}\right)=18.4^{\circ}$ if $R=10$ (arrows indicate the diffusion of trapped and passing ions in velocity space filling up the barrier; ions injected by the three pump beams $\angle E P B, M E P B$, and $H E P B$ are indicated in velocity space after acceleration by the potential well). 
The passing ions diffuse into the trapped region of velocity space and must be pumped out by the charge-exchange pumps. We must compute the diffusion rate and design the pumps accordingly.

Not all of the injected neutrals will be effective for charge-exchange pumping. Some of the neutrals will be ionized collisionally before they charge exchange, some will penetrate without collisions of any sort, and some will charge exchange with passing ions rather than with trapped ions. Correction factors for each of these effects must be included in the design.

TRAPPING RATE

Central-cell ions passing through the thermal barrier are trapped within the barrier by diffusion through velocity space by ion-ion multiple scattering. If the local mirror ratio $R(z)$ is large, the trapping is caused by pitch-angle diffusion, which is proportional to $R$. On the other hand, if $R$ is not much larger than unity, the trapping is caused by diffusion of $v_{\|}$, which is independent of $R$. An empirical equation combining these two effects, scaled from Fokker-Planck results, is

$$
J_{\text {trap }}=\frac{\mathrm{n}_{\text {pass }}^{2}}{5.5 \times 10^{9} \mathrm{~T}_{\mathrm{ic}}^{3 / \overline{2}}}[1+0.55 \mathrm{R}(\mathrm{z})] \text { trapped ions } / \mathrm{cm}^{3} \cdot \mathrm{s} \text {. }
$$

This must be integrated over the volume of the barrier, taking into consideration the reduction in $n_{\text {pass }}(z)$ as the ions are accelerated by the ambipolar potential and as the area of the flux tube expands in the reduced magnetic field. The total trapped ion current in each barrier is

$$
I_{\text {trap }}=2 q \int_{z_{b c}}^{z_{p}} J_{\text {trap }}(z) \frac{\pi r_{c}^{2} B_{c}\left(1-B_{c}\right)^{1 / 2}}{B(z)} d z \text { amperes . }
$$


In this integration, the passing-ion density $n_{\text {pass }}(z)$ and the potential $\psi^{*}(z)$ relative to ground can be modeled as follows:

$$
n_{p a s s}(z)=\left\{\begin{array}{l}
\frac{n_{c} B_{b}(z)}{B_{m b}}\left[-\frac{T_{i c}}{\pi\left[\phi_{e}-\phi^{*}(z)\right]+T_{i c}}\right]^{1 / 2} \text { for } \phi^{*}(z)<\phi_{e}, \\
\frac{n_{c} B_{b}(z)}{B_{m b}}\left[\frac{\exp -\left[\phi^{*}(z)-\phi_{e}\right] / T_{i c}-\exp \left(-\phi_{c} / T_{i c}\right)}{1-\exp \left(-\phi_{c} / T_{i c}\right)}\right] \text { for } \psi^{*}(z)>\phi_{e},
\end{array}\right.
$$

and

$$
\phi^{\prime}(z)=\left\{\begin{array}{l}
\phi_{e}-T_{e c} \ln \left[\frac{n_{c}}{g_{b} n_{p a s s}(z)}\right] \text { for } z_{b}^{c}=z_{b}=z_{b 0}, \\
\phi_{e}+\phi_{c}-T_{e p} \ln \left[\frac{n_{p}}{g_{b} n_{p a s s}(z)}\right] \text { for } z_{b 0}>z>z_{p}
\end{array} .\right.
$$

These profiles of density and potential are shown schematically in Fig. 2-1. For the magnet design of Chapter 3 the result of the above integration is $I_{\text {trap }}=1,076$ A for both ends of the TMR.

FOUR-STAGE PUMP ING SYSTEM

The power requirement for the pump beam, which may be substantial, is proportional to the beam energy $E_{B}$, which Eq. (5-1) shows to be at least equal to $\phi$. A large economy is possible by arranging conditions so that $\phi$ is as small as possible and perhaps even negative. This requires four pumping stages, similar in philosophy to a multi-stage differential pumping system for gas, in which most of the gas is pumped at a high pressure. Only a small. fraction of the gas load is pumped in the high-vacuum stage of a wel1-designed system. 
The four stages of the charge-exchange pump will be designated as the gas pump (GP), the low-energy pump beam (LEPB), the medium-energy pump beam (MEPB), and the high-energy pump beam (HEPB).

It is possible to use the gas-pump (GP) stage to pump out most of the trapped particles by charge exchange in the region where $\Delta \phi<0$ and therefore where $E_{B}$ may be zero. This charge-exchange pumping requires merely a gas jet directed into the appropriate plasma region--where $\phi^{*}(z)>\phi_{e}$ and where the plasma density is low enough Eor the gas to penetrate into the plasma. This region is in the low-density plasma surrounding the main plasma column of the thermal barrier. Computations ${ }^{5-1}$ indicate that trapped particles follow drift surfaces extending out of the barrier plasma because of asymmetric confinement by the plug quadrupole magnetic field. The fraction ${ } \mathrm{D}$ of the trapped ions on such drift surfaces can be pumped by the gas pump; i.e., $I_{G P}=E_{D} I_{\text {trap }}$.

Another large part of the trapped-ion population is accessible for charge exchange with a low-energy pump beam (LEPB) having sulficient speed merely to penetrate the barrier plasma to the $z$-axis before undergoing charge exchange or re-ionization. The only requirement for pumping by the LEPB is that the trapped ion be in the region of velocity space such that it spends part of its time in the region $\phi^{\star}(z)>\phi_{e}$. This requirement defines the fraction $\mathrm{F}_{\text {LEPB }}=I_{\text {LEPB }} / I_{\text {trap }}$. To economize on pump beam power, conditions should be arranged to maximize $f_{D}$ and $f_{L E P B}$.

The injection speed $v_{B}$ of the LEPB should be chosen so that a finite fraction of the injected neutrals reaches the z-axis but that a small fraction penetrates completely through the barrier; i.e.,

$$
1<n_{b} \times \overline{\sigma_{T} v} / v_{B}<4
$$


where $x=60 \mathrm{~cm}$ is the thickness of the plasma fan in the transition region, and the rate coefficient $\overline{\sigma_{\mathrm{T}} \mathrm{v}}$ for beam attenuation includes charge exchange and ionization by barrier ions and electrons. Since $v_{B}$ is smaller than the plasma thermal speeds, $\overline{\sigma_{T} v}=1$ to $2 \times 10^{-7} \mathrm{~cm}^{3} \cdot \mathrm{s} .5-2$ Therefore, for $\mathrm{n}_{\mathrm{b}}=1.4 \times 10^{13} \mathrm{~cm}^{-3}, \mathrm{v}_{\mathrm{B}}$ should be between 2 and $17 \times 10^{7} \mathrm{~cm} / \mathrm{s}$, depending on the plasma temperature and the desired pentration. We have chosen to use $20-\mathrm{kV}$ ion sources for the LEPB. To optimize the beam density and beam divergence, it is convenient to produce the LEPB from a mixture of molecular ions $\left(\mathrm{D}_{2}^{+}\right.$and $\left.\mathrm{D}_{3}^{+}\right)$.

It may be possible to use pellet injection rather than neutral beam injection to attain the desired penetration. However, this seems unlikely because of the high rate of pellet ablation at a 200-keV electron temperat ure.$^{5-3}$

Fokker-Planck computations show that if $g_{b}=2$, most of the trapped ions are accessible to charge-exchange pumping by the LEPB and GP because their trapped orbits extend to the region where $\phi^{*}(z)>\phi_{e}$ xigure $5-2$ shows contours of trapped and passing ions in velocity space for the condition in which $n_{\text {pass }}=n_{\text {trap }}$. Three hyperbolas loss surfaces are indicated, corresponding to the mirror ratio $R=10$ for passing ions, to the mirror ratio $R=2$ for a LEPB injected into a 6-T magnetic field with $v_{\|}>0$, and to the mirror ratio $R=3$ for the MEPB to be described.

The fraction $f_{L E P B}+F_{D}$ of trapped ions accessible for pumping by the GP and LEPB can be computed by integrating the contours between the two hyperbolas corresponding to the passing ions and to the injected LEPB. This integration results in $f_{L E P B}+F_{D}=0.77$.

To pump out the ions trapped more deeply in the potential well, it is necessary to inject neutral beams with sufficient energy $E_{B}$ to satisfy Eq. (5-1) and thereby escape from the barrier after the neutrals are converted 


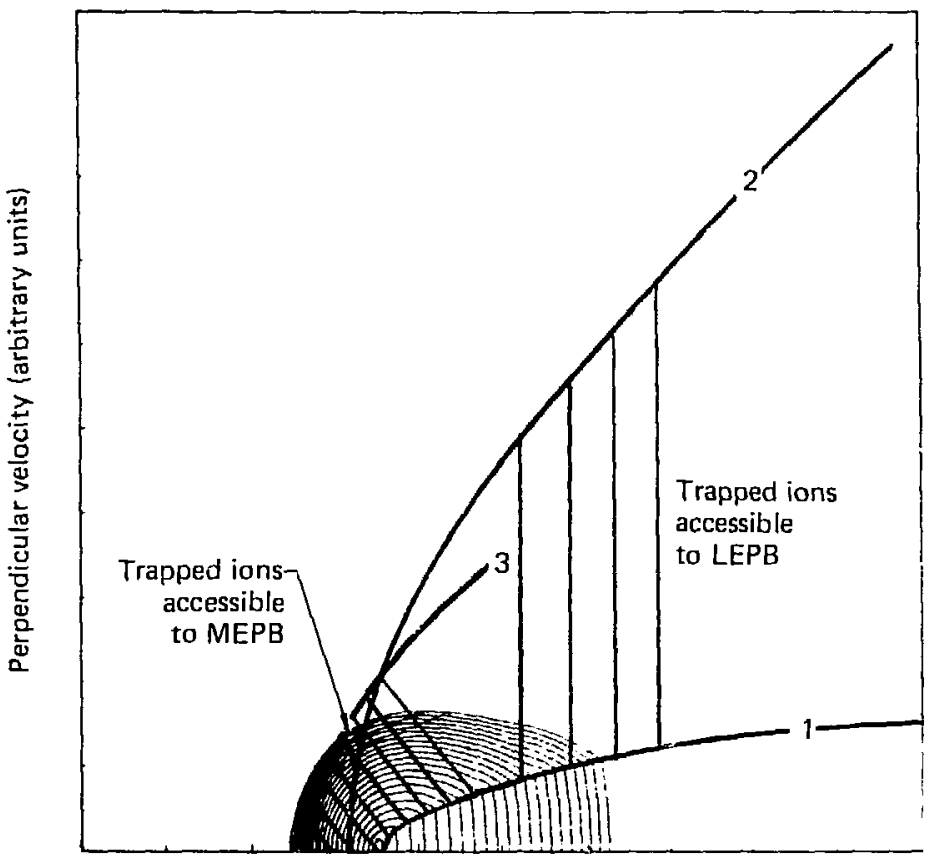

Parallel velocity (arbitrary units)

Fig. 5-2, Contours of ion density in velocity space, computed by a time-dependent Fokker-Planck code. The time is chosen such that $n_{\text {trap }}=n_{\text {pass }}$, such that $g_{b}=2$. The contours are plotted at equal logarithmic intervals, so that the ratio of densities for adjacent contours is 1.39. The three hyperbolas separate the trapped ions from the passing ions for three injection conditions: $1 . R=10$ for centra1-cell ions; 2. $R=2$ for LEPB ions injected into a 6-T magnetic field with $v \|>0$; and 3 . $R=3$ for MEPB ions injected into a 4-T magnetic field with $v_{\|}=\left(0.8 \phi_{b} q / m\right)^{1 / 2}$. The area in velocity space between hyperbolas 1 and 2 is accessible for charge-exchange pumping by the LEPB; the area between hyperbolas 1 and 3 is accessible to the MEPB.

to ions by charge exchange or by ionization. 'For each of the injected beams, a target area and a beam path must be selected so that $\phi, R$, and $\theta$ satisfy Eq. (5-1). For economy in beam power, it is best to pump as many trapped ions as possible at an intermediate potential $\triangle \dot{q}_{M E P B}$ with a MEPB and to pump only the ions trapped at the bottom of the potential well with a high-energy pump beam (HEPB) at the potential ${ }_{\text {HEPB }}=\phi_{b}=112 \mathrm{kV}$. 
We shall select $\Delta \phi_{\text {MEPB }}$ by using the requirement that $90 \%$ of the trapped ions not accessible to the LEPB be pumped by the MEPB; i.e., .

$$
\mathrm{f}_{\text {MEPB }}=I_{\text {MEPB }} / I_{\text {trap }}=0.9(1-0.77)=0.207 \text {, }
$$

and

$$
\mathrm{E}_{\mathrm{HEPB}}=\mathrm{I}_{\mathrm{HEPB}} / \mathrm{I}_{\text {traP }}=1-\left(\mathrm{f}_{\mathrm{D}}+\mathrm{f}_{\mathrm{LEPB}}+\mathrm{E}_{\mathrm{MEPB}}\right)=0.023 .
$$

We can obtain $\Delta \phi_{\text {MEPB }}$ from Fig. 5-2 by reading the value of $v_{\|}$at which the $10 \%$ density contour intersects the horizontal axis:

$$
\left(1-\Delta \phi_{M E P B}\right) / \phi_{b}=\left[v_{!}(10 \%) / v_{1}(100 \%)\right]^{2}=0.6 .
$$

Therefore

$$
\triangle \phi_{\text {MEPB }}=0.4 \phi_{b}
$$

Table 5-1 shows the values of $E_{B}$ and $\theta$-required to satisfy Eq. (5-1) For the MEPB and the HEPB, using the specified values of $\Delta \phi$ and $R$. We must Find injection paths satisfying these conditions.

TABLE 5-1. Beam parameters required to satisfy Eq. (5-1) for the medium-energy pump beam (MEPB) and the high-energy pump beam (HEPB).

\begin{tabular}{lrr}
\hline$\Delta \phi=\phi_{\mathrm{e}}-\phi^{*}, \mathrm{kV}$ & MEPB & HEPB \\
$\mathrm{R}=\mathrm{B}_{\mathrm{b}} / \mathrm{B}$ & 45 & 112 \\
Maximum $\theta$, degrees & 3 & 10 \\
$\mathrm{E}_{\mathrm{B}}=\phi /\left(1-\mathrm{R} \sin ^{2} \theta\right), \mathrm{keV}$ & 50 & 139 \\
\hline
\end{tabular}


For each of the four stages of the charge-exchange pump, the neutralbeam current requirement is derived from the pumping rate requirements ${ }_{G}{ }_{G P}$, $I_{\text {LEPB }}$, $I_{\text {MEPB }}$, and $I_{\text {HEPB }}$. The pumping requirements must be multiplied by three factors, $F_{1}, F_{2}$, and $F_{3}$, to take into account the interactions not contributing directly to charge-exchange pumping:

- Some of the injected neutrals will be ionized and will not undergo charge exchange. To allow for this, we must multiply by the ratio of rate coefficients $\overline{\sigma_{c x} v}$ and $\overline{\sigma_{i} v}$ averaged for the speed of the injected neutrals relative to the speed of the plasma ions and electrons. Interactions with both trapped ( $n_{\text {trap }}$ ) and passing ( $n_{\text {pass }}$ ) ions must be included. However, charge exchange with passing ions is not a loss because another neutral is produced which contributes to the charge-exchange pumping. The factor accounting for these effects is

$\begin{aligned} F_{1}= & \text { injected neutral beam/pumping rate (neglecting the } \\ & \text { losses due to beam penetration and re-ionization.) }\end{aligned}$

$$
\begin{aligned}
& =\frac{\left(n_{\text {trap }}+n_{\text {pass }}\right)}{\left.\frac{\left(\overline{\sigma_{c x} v}\right.}{n_{\text {trap }}}+\overline{\sigma_{i x} v}\right)-n_{\text {pass }} \overline{\sigma_{c x} v}} \\
& =1+\frac{g_{b} \overline{\sigma_{i} v}}{\left(g_{b}-1\right) \overline{\sigma_{c x} v}}
\end{aligned}
$$

where

$$
g_{b}=\left(n_{\text {trap }}+n_{\text {pass }}\right) / n_{\text {pass }} .
$$


- Equation (5-11) implicitly assumes that the entire injected beam undergoes either ionization or charge exchange. If this is not true, the injected current must be increased by a factor $F_{2}$ to compensate for the fraction penetrating without interactions.

$$
\mathrm{F}_{2}=\left\{\mathrm{l}-\exp \left[-\int \mathrm{dl}\left(\mathrm{n}_{\text {trap }}+\mathrm{n}_{\text {pass }}\right)\left(\overline{\sigma_{i} v}+\overline{\sigma_{c x} v}\right) / v_{B}\right]\right\}^{-1}
$$

- If a finite fraction of the charge-exchange products is re-ionized before escape from the barrier plasma, the neutral-beam requirement is increased by a factor $F_{3}$ equal to the reciprocal of the escape probability:

$$
F_{3}=\exp \left[\left(n_{\text {trap }}+n_{\text {pass }}\right) \overline{L \sigma_{i} / v}\right] \text {, }
$$

where the length of the escape path L depends upon the distance from the plasma surface $x \approx 10 \mathrm{~cm}$ and upon the two angles $\theta$ and $\phi:$

$$
L=X /(\sin \theta \sin \phi)
$$

The pitch angle $\theta$ of the trapped ion before charge exchange is roughly equal to the loss-cone angle $\sin ^{-1} \mathrm{R}^{-1 / 2}$. The azimuthal angle $\Phi$ is a random variable. We must compute the average value of $F_{3}$ as $\Phi$ varies randomly from 0 to 180 degrees. The result is that for the low density of this reactor example $\left(1.4 \times 10^{13} \mathrm{~cm}^{-3}\right)$, the exponent is small and $F_{3}$ is about 1.2 . For the gas pump in the very low density region, $F_{3}=1$.

In this computation, we neglect the possibility of a second charge exchange during the escape from the plasma. The second neutral product will contribute to the pumping and is not a loss.

In this section, we are assuming that each neutral beam consists of only one energy component (this assumption is discussed elsewhere). Therefore, the neutral-beam current requirement for each pumping stage is

$$
I^{0}=I_{\text {pumping }} \times F_{1} F_{2} F_{3}
$$


where the required pumping rate is

$$
I_{\text {putuping }}=I_{G P}, I_{L E P B}, I_{M E P B} \text {, or } I_{H E P B} \text {. }
$$

The computations for $\mathrm{I}^{0}$ for each stage are shown in Tables 5-2 through 5-5. In these computations, we specify the use of $T^{0}$ for the MEPB and HEPB to fuel the central cell and to take advantage of the better chargeexchange cross section.

\section{Choice of Beam Paths}

The location of the GP and LEPB is determined by the potential profile, such that $\phi^{*}$ is a few $\mathrm{kV}$ above $\phi_{e}$. This location is shown on the potential profile in Fig. 2-1 and in Fig. 1-4.

The beam paths for the MEPB and HEPB are chosen to fit several requirements:

- The beam path must be almost parallel to a magnetic flux line to satisfy Eq. (5-1).

- Each beam must be aimed at the target area where $\phi^{*}$ and $B$ have the values shown by Table 5-1. The potentials for the MEPB and HEPB are labeled on Fig. 2-1. Note that there are two possible locations for the MEPB injection.

- The useful part of the beam path must be stveral metres long so that most of the neutral beam is ionized or charge exchanged in the target region. Realistic conditions must be assumed for the beam density, beam access, and beam divergence. 
TABLE 5-2. Neutra1-beam requirements for each purping stage for the following specified parameters: $I_{\text {trap }}=1,076 \mathrm{~A}$ (both ends), $n_{\text {pass }}=7 \times 10^{12} \mathrm{~cm}^{-3}$, and $\mathrm{g}_{\mathrm{b}}=\mathrm{n}_{\mathrm{b}} / \mathrm{n}_{\text {pass }} \stackrel{\text { pass }}{=} 2$.

\begin{tabular}{|c|c|c|c|c|c|}
\hline & \multicolumn{5}{|c|}{ Pump stage } \\
\hline & $\mathbf{G P}$ & LEPB & MEPB & HEPB & Total \\
\hline$E_{B}$ (Erom Table 5-1) (kev/atom) & 0 & 10 & 50 & 139 & \\
\hline Injected species & $\mathrm{D}_{2}$ & $D^{0}$ & $\mathrm{D}^{0}, \mathrm{~T}^{0}$ & $\mathrm{~T}^{0}$ & \\
\hline Rate coefficients $\left(10^{-7} \mathrm{~cm}^{3} / \mathrm{s}\right):$ & & & & & \\
\hline $\overrightarrow{\sigma_{i} v}$ & 0.3 & 0.32 & 0.51 & 0.54 & \\
\hline$\overline{\sigma_{\mathrm{cx}} \mathrm{v}}$ & 1.4 & 1.4 & 0.80 & 0.31 & \\
\hline $\mathbf{f}$ & 0.4 & J.37 & 0.207 & 0.023 & 1.0 \\
\hline$I_{\text {pumping }}=\mp I_{\text {trap }}$ (A) & 430 & 398 & 223 & 25 & 1,076 \\
\hline$F_{1}$ [effect of ionization and & & & & & \\
\hline passing ions, Eq. $(5-11)]$ & 1.43 & 1.46 & 2.27 & 4.48 & \\
\hline $\mathrm{F}_{2}$ [attenuation factor, Eq. (5-12)] & 1.0 & 1.0 & 1.05 & 1.16 & \\
\hline $\mathrm{F}_{3}[$ escape factor, Eq. $(5-13)]$ & 1.0 & 1.2 & 1.2 & 1.2 & \\
\hline$I^{0}=F_{1} F_{2} F_{3} I_{\text {pumping }}$ & 615 & 697 & 639 & 156 & 2,107 \\
\hline Neutral beam power $=I^{0} E_{B}(M W)$ & 0 & 7.0 & 31.9 & 21.7 & 60.6 \\
\hline
\end{tabular}


It appears possible to satisfy these requirements by the beam paths indicated by Fig. 5-3, which shows the magnetic flux lines, and by Fig. 1-4, the scale drawing. Here we assume that in the final stage of a reactor design the details of the magnetic field will be reiterated to improve the conditions for magnetic pumping. Consequently, the correspondence between Fig. 5-3 and Table 5-1 is only approximate in this early stage of the design.

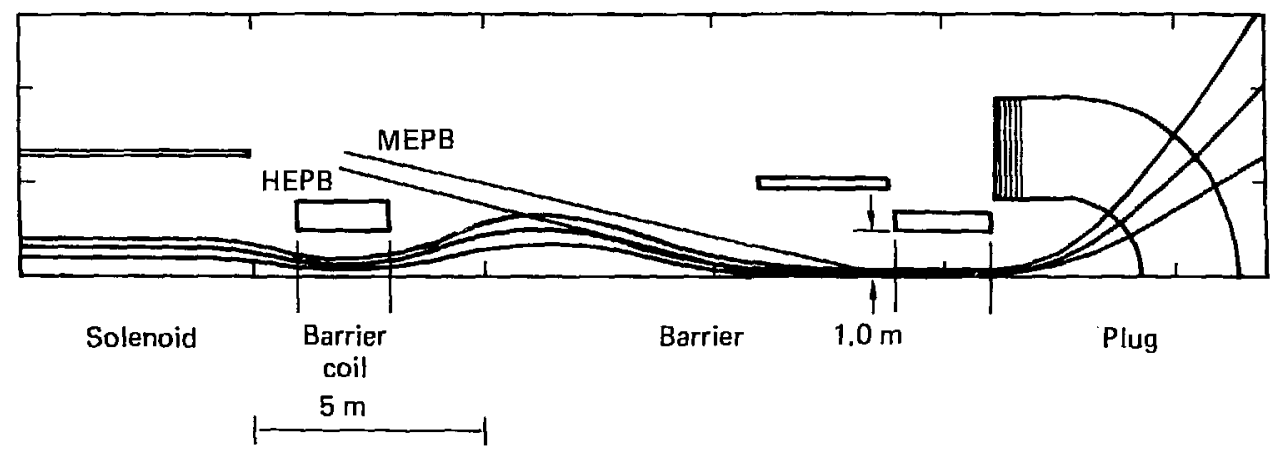

Fig. 5-3. Magnetic flux lines in the thermal barrier, with selected beam paths for MEPB and HEPB fulfilling the requirements of Table 5-1.

\section{Beam Purity Requirement}

Neutral beams derived from positive ions normally consist of a mixture of several energy components, derived from the mixture of $\mathrm{H}_{1}^{+}, \mathrm{H}_{2}^{+}$, and $\mathrm{H}_{3}^{+}$ in the primary beam. This causes a problem for the MEPB and HEPB because the half-energy and third-energy components will be trapped within the barrier if their energy is less than the required $E_{B}$ of Table 5-1. This problem does not affect the LEPB, for which the energy is not important except for plasma penetration. 
To indicate the severity of the problem, we shall compute the factor $\mathrm{F}_{4}$ defined as follows, withuut considering the correction factors $F_{1}, F_{2}$, and $\mathrm{F}_{3}$ :

$$
\begin{aligned}
& \mathrm{F}_{4}=\frac{\text { primary beam current }}{\text { pumpirig }}-\frac{-}{\text { seed of a mixed-energy neutal }}-\overline{\text { beam }}
\end{aligned}
$$

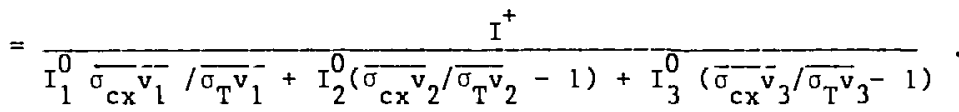

The subscripts 1,2 , and 3 denote the components derived from $\mathrm{T}_{1}^{+}, \mathrm{T}_{2}^{+}$, and $\mathrm{T}_{3}^{+}$. The subscripts $\mathrm{cx}$ and $\mathrm{T}$ denote the cross sections for charge exchange and for trapping the pump beam by all processes. The reaction rates ov are averaged for the relative speeds of a reactor plasma distribution. ${ }^{5-2}$

All three components contribute to the pumping mechanism by charge exchange. However, $\mathrm{I}_{2}^{0}$ and $\mathrm{I}_{3}^{0}$ also constitute a load that must be subtracted from the gross pumping speed.

The three neutral-beam components are related to the total pump beam $\mathrm{I}^{+}$as follows :

$$
I_{i}^{0}=n_{i} F_{i} I^{+} \times \text {atoms/ion }
$$

Here the composition of the primary beam is $\eta_{1}, \eta_{2}$, and $\eta_{3}$, and $F_{i}$ is the neutral fraction ${ }^{5-4}$ produced in an equilibrium neutralizing cell. Realistic values for the beam composition are 60-20-20\%. A more optimistic example is $80-15-5 \%$

Figure 5-4 shows $F_{4}$ computed as a function of $E_{B}$ for these two beam compositions and for a pure full-energy beam. The pumping speed is reduced by a large factor at energies in the range of the MEPB and becomes less than zero at energies in the range of the HEPB. Consequently, $F_{4}$ goes to infinity at high energy. 


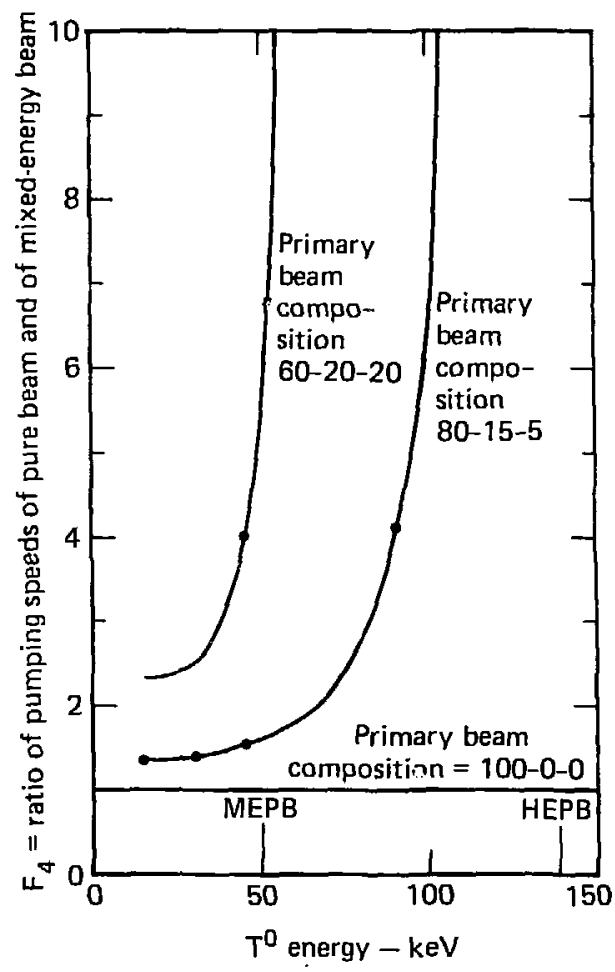

Fig. 5-4. Effect of half-energy and third-energy neutrals in chargeexchange pump beams. The effect becomes severe at higher energies, thus imposing a beam purity requirement.

It is clear that we must design beams for charge-exchange pumping without contamination by components of energy less than $E_{B}$. In principle, there are four ways to do this:

1. Development of a pure $T_{1}^{+}$plasma source is the most straightforward solution. However, we believe that a $90 \%$ pure source is the best attainable, and this is not good enough for the HEPB.

2. Neutral beams detived from $\mathrm{T}_{3}^{+}$will have no low-energy components. Howeyer, this implies an ion source voltage of $417 \mathrm{kV}$ for the HEPB. Also, there would be a large power cost because of the inefficient components derived from the $\mathrm{T}_{2}^{+}$and $\mathrm{T}_{1}^{+}$in the primary beam. 
3. Neutral beams derived from $\mathrm{T}^{-}$will have only one energy component. However, as shown in Chapter 4 the neutral-beam density is only $10 \mathrm{~mA} / \mathrm{cm}^{2}$ and the cost of $\mathrm{T}^{-}$production is $40 \mathrm{~kW}$ per injected ampere.

4. Magnetic separation of $\mathrm{T}_{1}^{+}$from the molecular ions in the primary beam before neutralization is possible. This can be done in a compact system by an iron-core deflection magnet in the highpressure $\left(10^{-3}\right.$ Torr) end of the neutralizer, implying a loss of $18 \%$ of the full-energy beam by premature charge exchange as the beam passes through the deflection magnet. This penalty seems preferable to the alternatives for the MEPB and HEPB.

The deflection magnet must be compact to minimize the beam loss by premature charge exchange. This is possible if the deflection angle is not large. As shown in Fig. 5-5, it is sufficient to deflect the $\mathrm{T}_{1}^{+}$by 10 degrees to separate the $\mathrm{T}_{1}^{+}$from the 7.1 degree deflection of the $\mathrm{T}_{2}^{+}$and from the 5.8 degree deflection of the $\mathrm{T}_{3}^{+}$. The required deflection field is

$$
\begin{aligned}
\int \mathrm{B}_{\perp} \mathrm{d} 1=\left(2 \mathrm{mE} \mathrm{E}_{\mathrm{B}} / \mathrm{q}\right)^{1 / 2} & =0.016 \mathrm{~T} \cdot \mathrm{m} \text { for the HEPB } \\
\text { of } & =0.010 \mathrm{~T} \cdot \mathrm{m} \text { for the MEPB } .
\end{aligned}
$$

This deflection requires a magnetic field strength of only $0.08 \mathrm{~T}$ if the thickness of the magnetic field is $20 \mathrm{~cm}$. Including the fringing fields, the overall thickness will be about $40 \mathrm{~cm}$. Figure 5-5 shows the deflection magnet and neutralizer to scale in two views. The deflection magnets are viewed from the side in Fig. $1-4$ and therefore are not visible.

The same design can be used for the HEPB and for the MEPB. the conductance of the neutralizer must be adjusted so the gas pressure is a maximum of $10^{-3}$ Torr for the HEPB and $0.4 \times 10^{-3}$ Torr for the LEPB. These gas pressures will result in the same $18 \%$ penalty for both beams. 
(a)

Collimator for $T^{0}$ derived from $T_{1}^{+}$ Acceptance angle $=10^{\circ} \pm 1.5^{\circ}$

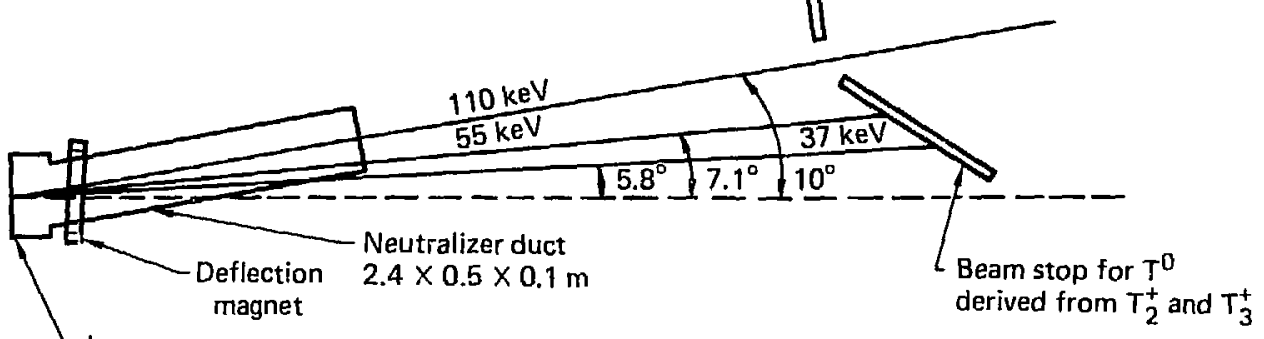

lon source

(plan view)

6-m drift from deflection magnet to collimator

(b)

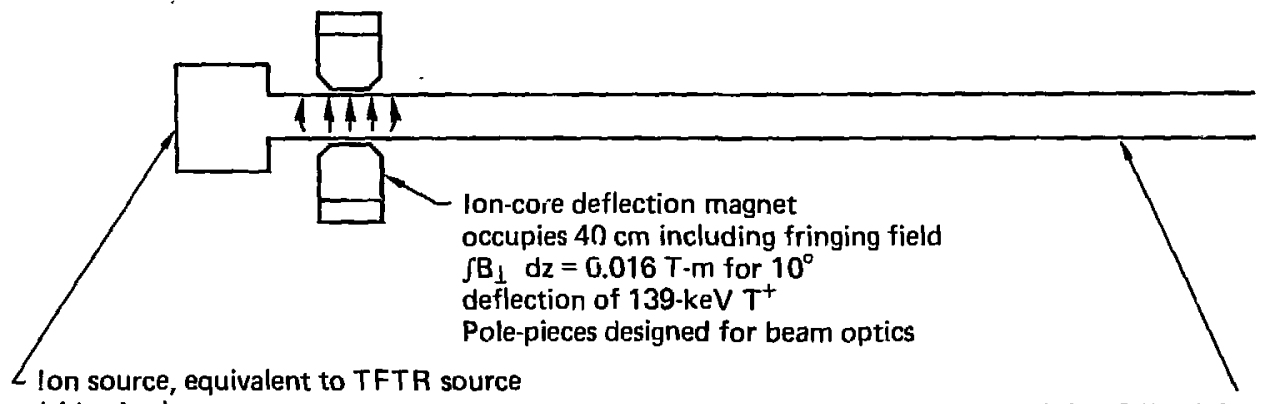

(side view)

$110-\mathrm{kV}, 50-\mathrm{A}$ total beam $/ \mathrm{module}$

$80 \% \mathrm{~T}_{1}^{+} ; 20 \% \mathrm{~T}_{2}^{+}$and $\mathrm{T}_{3}^{+}$

Neutralizer duct, about $2.4 \times 0.5 \times 0.1 \mathrm{~m}$

Gas pressure varies lineaily from

$10^{-3}$ to $10^{-5}$ Torr

Fig. 5-5. Magnetic deflection of the primary beam before neutralization: (a) plan view, showing products of $\mathrm{T}_{1}^{+}$separated from products of $\mathrm{T}_{2}^{+}$and $\mathrm{T}_{3}^{+}$by a 10-degree magnetic deflection; and (b) side view, showing a compact iron-core deflection magnet at the high-pressure end of the neutralizer duct. 
To attain a neutralization fraction $90 \%$ of the equilibrium fraction, the length of the neutralizer after the deflection magnet will be $-2 \mathrm{~m}$ for both the HEPB and the LEPB. This results in $54 \%$ neutralization of the HEPB and $82 \%$ neutralization of the MEPB.

\section{Reactor Fueling}

In the example of Table $5-2$, all the neutral pump beams are converted to ions and injected into the central cell as reactor fuel except for the fraction that penetrates through the plasma without ionization or charge exchange. The slow ions produced by the gas pump will not enter the central cell because they are born without kinetic energy outside the dense plasma and will follow flux lines to a collector. The reactor fuel injected by each beam is $\mathrm{I}^{0} / \mathrm{F}_{2}$, shown by Table 5-3 to be a total of $1,440 \mathrm{~A}$. It will be convenient to adjust conditions so that this injected current fulfills $100 \%$ of the reactor fueling requirements. In the next step of the reactor design, the parameters $f_{D}, f_{\text {LEPB }}$, $F_{M E P B}, f_{H E P B}$, the gas pump baffles, and perhaps the length of the central cell will be adjusted to equalize the fueling requirement and the injected pump beam current. This equalization will be deferred until the next design stage when we will have a fully consistent design. The injected currents of $\mathrm{D}^{+}$and $\mathrm{T}^{+}$should be proportional to the rate of Coulomb scattering to equalize the densities of $\mathrm{D}^{+}$and $\mathrm{T}^{+}$in the central-cell plasma. The Coulomb scattering rate is inversely proportional to the ion mass, since both species have the same temperature and charge. We have specified that the HEPB and most of the MEPB be $100 \% \mathrm{r}^{0}$ to take advantage of the larger charge-exchange cross section. Table 5-3 shows how the injected currents can be adjusted to fulfill these requirements. 
TABLE 5-3. Reactor fueling by charge-exchange pump beams.

GT $\overline{\text { LEPB }}$ MEPB

Total

Current injected into central ce $11, \mathrm{I}^{0} / \mathrm{F}_{2}$ (A)

0

697

609

134

1,440

$\mathrm{T}^{+}$injected (A)

0

0

513

134

647

$D^{+}$injected (A)

0

697

96

0

793

TABLE 5-4. Cold-electron load (both ends).

\begin{tabular}{|c|c|c|c|c|c|}
\hline & GP & LEPB & MEPB & HEPB & Total \\
\hline $\begin{array}{l}\left(F_{1} F_{2} F_{3}\right)^{-1} \text { calculated } \\
\text { from Table 5-2 }\end{array}$ & 0.699 & 0.571 & 0.350 & 0.160 & \\
\hline $\begin{array}{l}\left(1-F_{2}^{1}\right) \text { calculated } \\
\text { from Table 5-2 }\end{array}$ & 0 & 0 & 0.048 & 0.138 & \\
\hline I $_{\text {ionization }}($ Eq. 5-18) (A) & 185 & 299 & 385 & 110 & 979 \\
\hline Electron current into plug (A) & 0 & 299 & 385 & 110 & 794 \\
\hline Potential difference $(\mathrm{kV})$ & 112 & 112 & 67 & 0 & \\
\hline Cold-electron power load, a (MW) & 0 & 33.5 & 25.8 & 0 & 59.3 \\
\hline
\end{tabular}

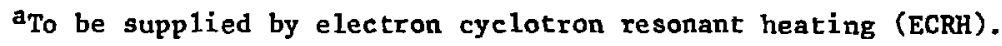


The cold electrons produced by ionization of injected neutrals will be a load on the electron energy balance. If the cold electrons enter the plug plasma, they will create a load for electron cyclotron resonant heating (ECRH). If they enter the central cell, they will be heated indirectly by collisions with alpha particles.

The rate of electron production ( $I_{\text {ionization }}$ ) by ionization of each of the neutral beams $I^{0}$ can be computed by the factors $F_{1}, F_{2}, F_{3}$. The injected neutral beam can be divided into three fractions: charge-exchange pumping, ionization, or penetration through the plasma:

$$
\mathrm{I}^{0}=\mathrm{I}_{\text {pumping }}+\mathrm{I}_{\text {ionization }}+\mathrm{I}_{\text {penetration }}
$$

where

$$
\mathrm{I}_{\text {pumping }}=\mathrm{I}^{0} / \mathrm{F}_{1} \mathrm{~F}_{2} \mathrm{~F}_{3}
$$

and

$$
I_{\text {penetration }}=I^{0}\left(1-F_{2}^{-1}\right)
$$

Consequent1y,

$$
I_{\text {ionization }}=I^{0}\left[1-\left(F_{1} F_{2} F_{3}\right)^{-1}-\left(1-F_{2}^{-1}\right)\right] \text {. }
$$

The results of this computation are shown in Table 5-4, with the additional assumption that electrons produced by the gas pump can be baffled from the plug plasma. 
Electrons produced by the HEPB at the bottom of the potential well have potential energy of $\phi_{b}+\phi_{c}$ relative to the plug plasma and require no further heating. Electrons produced by the MEPB have potential energy of only $0.4 \phi_{b}+\phi_{c}$, while electrons produced by the LEPB have potential energy of $\phi_{c}$. The ECRH load it determined by the need to heat the cold electrons to a rotal energy of $\phi_{b}+\phi_{c}$. This computation is also shown in Table 5-4.

\section{Charged Beam Power}

In Table 5-5, we compute the charged beam power and the total input power required for the charge-exchange beams. This computation is based on the neutra1-beam power $E_{B} I^{0}$ for each pump beam as computed in Table 5-2. The correction factors $F_{5}, F_{6}$, and $F_{7}$ are required to account for the beam fractions lost because of the neutralization, the nonuseable molecular ions in the primary beam, and the premature charge exchange in the deflection magnet. These losses have been discussed in other sections of this chapter. The result is that for this example the total input power required for the pump beams is $118.9 \mathrm{MWe}$, of which $56.1 \mathrm{MW}$ is collected by beam dumps in the injectors and is available for thermal or electrical energy recovery. If the efficiency of themal energy recovery is $30 \%$, the net input power is 101.2 MWe. A further saving of about $10 \mathrm{MW}$ is possible by using beam direct recovery where this is practical rather than thermal energy recovery. Beam direct recovery at $70 \%$ efficiency has been shown to be practical in experiments at LLL/LBL, ORNL, and at Fontenaymaux-Roses under conditions similar to those of the MEPB and HEPB. 
TABLE 5-5. Charged beam power requirement.

\begin{tabular}{|c|c|c|c|c|c|c|}
\hline & $\overline{G P}$ & & IEPB & $\overline{M E} \overline{P B}$ & $\overline{\mathrm{HE}} \overline{\mathrm{PB}}$ & Tota1 \\
\hline Neutralization fraction, $F_{5}$ & 1.0 & & 0.88 & 0.82 & 0.54 & \\
\hline Fraction in usable specie, $F_{6}$ & 1.0 & & 1.0 & 0.9 & 0.9 & \\
\hline Fraction lost in deflection, $F_{7}$ & 0 & & 0 & 0.18 & 0.18 & \\
\hline $\mathrm{I}^{+}=\mathrm{I}^{0} / \mathrm{F}_{5} \mathrm{~F}_{6}\left(1-\mathrm{F}_{7}\right)$ & -- & or & $\begin{array}{lll}792 & \mathrm{Al}^{+} \\
396 & \mathrm{~A} & \mathrm{D}_{2}^{+}\end{array}$ & 1,056 & 391 & 1,843 \\
\hline $\begin{array}{l}\text { Charged beam power, } \\
\mathrm{I}^{+} \mathrm{E}_{\mathrm{B}} \text { (MWe) }\end{array}$ & 0 & & 7.9 & 52.8 & 54.5 & 115.2 \\
\hline $\begin{array}{l}\text { Arc and filament power, } \\
2 \mathrm{keV} / \text { ion extracted (MWe) }\end{array}$ & 0 & & 0.8 & 2.1 & 0.8 & 3.7 \\
\hline Total pump beam input power (MWe) & 0 & & 8.7 & 54.9 & 55.3 & 118.9 \\
\hline $\begin{array}{l}\text { Number of neutral-beam modules } \\
\text { requi red, both ends, based on } \\
\mathrm{I}^{+}=60 \mathrm{~A} / \text { module }\end{array}$ & 0 & & 6 & 18 & 8 & 32 \\
\hline $\begin{array}{l}\text { Ion beam power available for } \\
\text { energy recovery in the } \\
\text { injector, (MWt) }\end{array}$ & 0 & & 0.84 & 20.8 & 34.5 & 56.1 \\
\hline $\begin{array}{l}\text { Net pump beam input power af ter } \\
\text { thermal energy recovery at } \\
30 \% \text { efficiency (MWe) }\end{array}$ & 0 & & 8.4 & 48.6 & 44.2 & 101.2 \\
\hline $\begin{array}{l}\text { Beam power available for } \\
\text { direct conversion, } \\
\text { full-energy charged beam } \\
\text { only, I0/F }-I^{0} \text { (MWe) }\end{array}$ & 0 & & 0 & 7.0 & 18.5 & 26.5 \\
\hline $\begin{array}{l}\text { Net pump beam input power after } \\
\text { beam direct conversion at } 70 \% \\
\text { and thermal conversion at } 30 \% \\
\text { efficiency (MWe) }\end{array}$ & 0 & & 8.4 & 45.9 & 37.5 & 91.8 \\
\hline
\end{tabular}




\section{REFERENCES}

5-1. J. Kesner, Passive Generation of Ambipolar Potential Barriers in a Tandem Mirror, University of Wisconsin, Rept. UWFDM-303; to be pub1ished in Comments on Plasma Physics and Controlled Fusion.

5-2. A. C. Riviere, "Penetration of Fast Hydrogen Atoms into a Fusion Reactor P1asma", Nuc1. Fusion 11, 363 (1971).

5-3. S. L. Milora and C. A. Foster, "A Revised Neutral Gas Shielding Model for Pellet-Plasma Interactions", IEEE Tran. Plasma Sci., Ps-6, 578 (1978).

5-4. C. F. Barnett, J. A. Ray, E. Ricci, and M. I. Wilker, Atomic Data for Controlled Fusion Research, Oak Ridge National Laboratory, Rept. ORNL-5206, Vol. I, p. A.6.2 (1977). 
CHAPTER 6. ELECTRON CYCLOTRON RESONANT HEATING

G. W. Hamilton, B. Arfin

Introduction . . . . . . . . . . . . 151

Choice of Frequency and Resonant Magnetic Field . . 152

Microwave Polarization and Absorption . . . . . 153

ECRH Power Requirements . . . . . . . . . . 155

Waveguide Limitations . . . . . . . . . . 156

Gyrotron Radio Frequency Sources . . . . . . . 158

Appendix. Calculation of Maximum Power

That Can Be Carried in a Waveguide . . . . . . . . 160

References . . . . . . . . . . . . 162 
INTRODUCTION

Some means of heating the electrons up to $200 \mathrm{keV}$ in the plug is required to increase the ambipolar potential confining the central cell ions. The available plasma heating techniques are nectral-beam injection, electron beam heating, and several types of radio frequency ( $r f$ ) heating. ${ }^{-1}$ our design studies show that the thermal barrier greatly reduces the heating requirements because the barrier makes it possible to heat the plug plasma without also heating the large central cell.

Neutral-beam requirements are significantly reduced by If heating, which reduces the electron drag. In this design the $400-k e V$ neutral beams are required only to provide the fast ions required for plug confinement. Plug electrons are heated primarily by $r f$. We feel that not enough information is available on the absorption of electron beams in the end-plug plasma for a reactor design.

Four types of rf heating are available for consideration:

- Alfven wave heating 1-10 $\mathrm{MHz}$

- Ion cyclotron resonant heating (ICRH) $50-100 \mathrm{MHz}$

- Lower hybrid resonant heating (LHRH) $\quad 1-5 \mathrm{GHz}$

- Electron cyclotron resonant heating (ECRH) $30-150 \mathrm{GHz}$

In general, the cost per watt increases as a weak function of frequency. ${ }^{6-2}$ However, we prefer ECRH for this application for several reasons:

- ECRH transfers the rf power to the electrons; such a transfer is our objective.

- More experimenta1 data are available for $\mathrm{EGRH}^{6-3}$ than for the other types of $\mathrm{rf}$ heating. 
- ECRH power can be transmitted by simple waveguides, requiring no special antenna systems if polarization is not important.

- High-power oscillators (gyrotrons) in the ECRH frequency range have recently become available; this development makes it possible to extrapolate the performance of existing rf systems to the leve1s required by TMR without the need for totally new inventions.

CHOICE OF FREQUENCY AND RESONANT MAGNETIC FIELD

Efficient transfer of rf power to a plasma normally requires coupling with one of the fundamental plasma resonances. In ECRH the resonant frequency is the fundamental or a harmonic of the electron cyclotron frequency; i.e.,

$$
f=f_{c e} \text { or } 2 f_{c e}
$$

where

$$
f_{c e}=e B / 2 \pi m_{e}=28 B\left[f_{c e} \text { in } \mathrm{GHz}, \mathrm{B} \text { in Teslas }\right] .
$$

However, the resonant condition must occur where the plasma is accessible to the injected $\mathrm{r} f$ power. This requires that the microwave frequency be higher than the electron plasma frequency to avoid reflections from the plasma cutoff; i.e.,

$$
\mathbf{f}>\mathbf{f}_{\text {pe }}
$$

where

$$
f_{p e}=(1 / 2 \pi)\left(n_{e} e^{2 / m} \varepsilon_{0}\right)^{1 / 2}=8,976 n_{e}^{1 / 2}\left[f_{p e} \text { in } H z, n_{e} \text { in cm } c^{-3}\right] \cdot(6-2)
$$

Fortunately, Conditions (6-1) and (6-2) are mutually compatible in the TMR plugs, where the maximum density is $\mathrm{n}_{\mathrm{e}}=3 \times 10^{13} \mathrm{~cm}^{-3}$. Therefore, $f_{p e} \leq 49 \mathrm{GHz}$ and the Conditions $(6-1)$ and $(6-2)$ may be satisfied if $B$ is 
larger than $1.7 \mathrm{~T}$. These requirements may be satisfied either in the plug itself or in the transition region between the plug and the thermal barrier. The electrons in the transition region are electrostatically trapped by the positive plug potential and belong to the same population as the plug electrons. The transition region is convenient for installation of wave guides because space is available between the magnet conductors.

Some other practical considerations affecting the choice of frequency are the magnetic field required for other reasons and the state-of-the-art of high-frequency gyrotrons. In order to heat the transition region near the plug and to avoid heating the central cell electrons, we will design Eor $B>2 \mathrm{~T}$ and $\mathrm{f}_{\mathrm{ce}}>56 \mathrm{MHz}$. To avoid a requirement for gyrotron frequencies higher than $100 \mathrm{MHz}$ we will use B < $3.6 \mathrm{~T}$ for ECRH. These conditions can be satisfied in the transition region in the following range:

Range of conditions for ECRH in the transition region:

$$
\begin{array}{ll}
2<\mathrm{B}<3.6 \mathrm{~T} & \mathrm{n}_{\mathrm{e}}=2.2 \times 10^{13} \mathrm{~cm}^{-3} \\
56<\mathrm{f}_{\mathrm{ce}}<100 \mathrm{GHz} & \mathrm{E}_{\mathrm{pe}}=42 \mathrm{GHz}
\end{array}
$$

MICROWAVE POLARIZATION AND ABSORPTION

Conditions must be selected such that the microwaves injected into the plasma will be strongly absorbed either in a single transit through the resonant zone or by repeated transits within a cavity with reflecting boundaries. In TMR it is not possible to enclose the transition plasma completely within a cavity. However, we can show that the microwaves will be almost $100 \%$ absorbed by the plasma electrons in a $\sin _{\overline{5}}$ le pass.

If we use circular waveguides for microwave injection, approximately $50 \%$ of the injected power will be in the ordinary mode for which the planepolarized rf electric field is parallel to the B field; and $50 \%$ of the power 
will be in the extraordinary mode for which the rf electric field is perpendicular to the B field. The extraordinary mode is absorbed much more strongly than the ordinary mode because the electrons are less free to move with the rf electric field. However, because of the high density and temperature of the TMR electrons, even the ordinary mode will be totally absorbed in a single transit. This condition can be seen from Porkolab's absorption theory. ${ }^{6-3}$ The absorption coefficient $\Gamma_{0}$ is defined as $T=\exp \left(-\Gamma_{0}\right)$ where $T$ is the transmitted fraction. Porkolab's results for the ordinary mode are:

$$
\begin{aligned}
\Gamma_{0} & =2 \pi \mathrm{L}_{B}(\omega / c)\left(\mathrm{T}_{\mathrm{e}} / \mathrm{m}_{\mathrm{e}} \mathrm{c}^{2}\right) Q_{0} \\
& =2.56 \times 10^{-6} \mathrm{~L}_{B}(\mathrm{~cm}) \mathrm{f}(\mathrm{GHz}) \mathrm{T}_{\mathrm{e}}(\mathrm{eV}) \mathrm{Q}_{0},
\end{aligned}
$$

where

$$
\begin{aligned}
& Q_{0}=\frac{1}{4} \frac{\alpha^{2}\left(1-\alpha^{2}\right)^{1 / 2}}{1+N_{z}^{2}\left(1-\alpha^{2}\right)}, \\
& \alpha^{2}=f_{p e}^{2} / f^{2}<1, \\
& N_{z}=c k_{z} / \omega_{0} \ll 1 \text { for } k \perp B \sim,
\end{aligned}
$$

and

$$
L_{B}=|B(d B / d x)|^{-1}
$$

The component of the index of refraction along the magnetic field, $N_{z}$, is small if $\underset{\sim}{\mathrm{k}}$ is perpendicular to $\underset{\sim}{\mathrm{B}} . \mathrm{N}_{\mathrm{z}}$ does not strongly affect $\mathrm{Q}_{0}$ even if $\mathrm{N}_{2}$ is not small. Also, $L_{B}$, the scale length of the magnetic field gradient at the resonant layer, is 100 to $500 \mathrm{~cm}$ in the TMR transition region. In respect to absorption of microwaves, the worst condition will be at the time of startup when the plasma is sustained only by the injected neutral 
beams. The absorption parameters during startup and during TMR operation are shown in Table 6-1.

TABLE 6-1. ECRH absorption parameters in the transition plasma.

\begin{tabular}{|c|c|c|}
\hline & Startup & Operation \\
\hline $\mathrm{n}_{e}, \mathrm{~cm}^{-3}$ & $0.5 \times 10^{13}$ & $2.2 \times 10^{13}$ \\
\hline $\mathrm{T}_{\mathrm{e}}, \mathrm{keV}$ & 10 & 200 \\
\hline $\mathrm{E}_{\mathrm{pe}}, \mathrm{GHz}$ & 20 & 42 \\
\hline$F=56$ to $100 \mathrm{GHz}$, dep & only upon $B$. & \\
\hline$\alpha=\mathrm{f}_{\mathrm{pe}} / \mathrm{f}$ & 0.2 to 0.36 & 0.42 to 0.75 \\
\hline $\mathrm{Q}_{0}$ & 0.01 to 0.05 & 0.05 to 0.095 \\
\hline $\begin{array}{l}\text { Minimum value of } \Gamma_{0} \\
\quad \text { if } L_{B}=200 \mathrm{~cm}\end{array}$ & 5.12 & 25.6 \\
\hline$T=\exp \left(-\Gamma_{0}\right)$, maximum & $6 \times 10^{-3}$ & $10^{-11}$ \\
\hline
\end{tabular}

This computation indicates that absorption in a single transit will be no problem in TMR even under startup conditions. A mixture of wave modes and polarizations can be injected with efficient absorption. This conclusion is supported by reports on recent experiments in ECRH. ${ }^{6-4}$

ECRH POWER REQUIREMENTS

There are two categories of ECRH power requirements:

1. The largest portion of ECRH power is needed to heat the cold electrons produced by ionization of the charge-exchange beams used to pump the trapped ions out of the thermal barriers. In Table 5-4 of Chapter 5 this is computed as $30 \mathrm{MW}$ for each thermal barrier. 
2. The second requirement for ECRH heating is the maintenance of the 200-keV electron temperature in the plugs and transition region. This requirement is computed as $9 \mathrm{MW}$ for each end of TMR, based upon the power transfer from the hot plug electrons to the cooler center cell electrons and upon the power loss by synchrotron radiation.

The total requirement for ECRH power is therefore 39 MW at each end of TMR. This requirement represents an increase over existing technology that is surely attainable during the next two decades.

WAVEGUIDE LIMITATIONS

Figure 1-4 is a layout indicating an assembly of waveguides delivering ECRH power to the transition region of TMR. Each waveguide has a diameter equal to 20 to 80 wavelengths of the radiation at frequencies in the range from 56 to $100 \mathrm{GHz}$. In the present state-of-the-art the capacity of each waveguide is limited by the power absorbed by the windows, which consist of two layers of dielectric cooled by a liquid fluorocarbon flowing between the 1ayers.

It is necessary to make a design decision of whether to design within the 1 imitations of If windows or to design the waveguides to operate under vacuum without windows but with differential pumping to maintain the high vacuum ( $10^{-8}$ Torr) required for gyrotron operation. It appears that both approaches are feasible, but it is difficult to predict at this time which approach is better. In Table 6-2 we present the waveguide parameters suitable for operation of the ECRH system both with and without windows. Both examples appear to be feasible with moderate improvements of existing technology. 
TABLE 6-2. Two examples of ECRH parameters for TMR.

Wich rf windows Without windows

Total ECRH power to each end, MW 39 39

Power density in waveguides, $\mathrm{kW} / \mathrm{cm}^{2}$ 2 10

Gyrotron power output, each, MW

Number of gyrotrons required 39 20

Waveguide diameter, $\mathrm{cm}$ 25 16

Overa11 efficiency, \% 60 60

Gyrotron input power, MW total , MW each 60 1.7 60

Gyrotron electron beam, $\mathrm{kV}$

Several factors must be considered in the decision whether to use waveguide windows. A window at the tube is an asset for two reasons: (1) to enable the manufacturer to test the tube before shipment; and (2) to protect the tube from damage when the reactor vacuum is vented.

Windows would be needed at both ends of the waveguide if the waveguide were pressurized to avoid $\mathrm{rf}$ breakdowns. Such breakdowns are expected if the waveguide under low vacuum must penetrate the fringing field of TMR in a condition such that $f=\mathrm{f}_{c e}$. However, this problem can be avoided by other means-by evacuating the waveguide to high vacuum or by avoiding the condition $f=f_{c e}$ within the waveguide. Injection of rf into the non-minimum-B transition region is convenient in this respect because there is only one radial position in a non-minimum-B field where $f=f_{c e}$, while there are two such positions in a minimum-B field. 
It may be difficult to shield an if window near a reactor from neutrons and other types of bombardment. The window location must be protected by neutron shielding and by a miter-bend so the window is not directly exposed to radiation and particles emitted by the reactor plasma.

If the $\mathrm{rf}$ window is eliminated the complications involved are a differen- tial pumping system to maintain a vacuum at $10^{-8}$ Torr and a gate valve that may be closed to isolate the gyrotron from the waveguide. If the differential pumping system is cryogenic, it must be somehow protected from heating by stray rf. These complications do not seem impossible to solve.

In the Appendix to this chapter a computation is shown to demonstrate that the average microwave power density specified by this design is at least one order of magnitude less than the ultimate power density limitation imposed by $\mathrm{rf}$ breakdown.

GYROTRON RADIO FREQUENCY SOURCES

The recent development of gyrotron oscillators with continuous outputs up to $200 \mathrm{~kW}$ at $28 \mathrm{GHz}$ has improved the feasibility of ECRH at the power levels required for fusion reactors. The gyrotron oscillator converts power from an electron beam to rf oscillations by passing the beam through a resonant cavity in a magnetic field tuned such that the electron cyclotron frequency resonates with one of the cavity frequencies. ${ }^{6-5}$ The cavity dimensions may be much larger than the wavelength if high mode numbers are used for the cavity resonance. The relativaly large cavity dimensions permit high output powers before the limitations associated with high power densities become critical. 
A pulsed gyrotron has been reported to deliver $1.1 \mathrm{MW}$ at $100 \mathrm{GHz}$ for $100 \mathrm{ss}^{6-6}$ This performance would fulfill the TMR requirements if the pulse length and efficiency were improved. Efficiencies of 30 to $45 \%$ have been measured in other installations; these can probably be improved to the $60 \%$ overall efficiency assumed in Table 6-2.

We expect the capital cost of a large ECRH system to be about $\$ 1.00 / \mathrm{W}$ delivered to the plasma. This is a rate competitive with the capital costs of neutra1-beam systems. This estimate is based on comparisons of the ECRH components with neutra1-beam components and the components of other large power systems. Costs of existing gyrotrons are not relevant to gyrotron costs for a reactor because of the development expenses that will not apply to a reactor. 
The maximum power density that can be transmitted along a circular waveguide will be found as a function of the maximum allowable peak fields. The general formula will be derived for the $\mathrm{TE}_{\text {on }}$ modes and then the power in the $\mathrm{TE}_{01}$ and $\mathrm{TE}_{02}$ modes will be found for a peak field of $29 \mathrm{kV} / \mathrm{cm}$.

The fields for the $\mathrm{TE}_{\text {on }}$ modes can be written as:

$$
\begin{aligned}
& H_{z}=H_{0} J_{0}(k r) e^{j(\omega t-\beta z)}, \\
& H_{r}=\frac{j B H_{0}}{k} J_{1}(k r) e^{j(\omega t-\beta z)}, \\
& E=\frac{j \omega \mu H_{0}}{k} J_{1}(k r) e^{j(\omega t-\beta z)},
\end{aligned}
$$

where:

$$
\begin{aligned}
& \mathrm{k}=\frac{2 \pi}{\lambda_{\mathrm{c}}}=\mathrm{p} / \mathrm{b}, \\
& \beta^{2}=\omega^{2} \mu E-\mathrm{k}^{2}, \\
& \mathrm{p}=3.82,7.016 \text { for } \mathrm{TE}_{01}, \mathrm{TE}_{02}, \\
& \lambda \text { is the free space wavelength, } \\
& \mathrm{b} \text { is the guide radius in } \mathrm{cm} .
\end{aligned}
$$

The power transmitted can be found by use of Poynting's vector:

$$
P_{z}=\frac{1}{2} \operatorname{Real} \int_{\theta=0}^{\theta=2 \pi} \int_{r=0}^{r=b} E_{0} H_{r}^{*} r d \theta d r .
$$


This $c$ an be shown to be

$$
\frac{P_{z}}{\pi b^{2}}=1.326 \times 10^{-3} \frac{\lambda}{\lambda_{g}} \frac{J_{0}^{2}(k b)}{J_{1}^{2}(k r)_{\max }} \mathrm{E}_{0}^{2} \mathrm{~W} / \mathrm{cm}^{2}
$$

where

$$
\begin{aligned}
& \lambda_{\mathrm{g}} \text { is the guide wavelength, } \\
& \mathrm{E}_{0} \text { is the maximum field in } \mathrm{V} / \mathrm{cm} .
\end{aligned}
$$

The maximum value of the rf electric field limited by breakdown in air has been measured as $\mathrm{E}_{0}=2.9 \times 10^{4} \mathrm{~V} / \mathrm{cm} .^{6 \mathrm{~A}-1}$ In vacuum or in pressurized gas $\mathrm{E}_{0}$ would be more than this. The ratio $\lambda / \lambda_{\mathrm{g}}$ is almost unity for an oversize waveguide. The Bessel functions $J_{0}(k b)$ and $J_{1}(k r)$ depend upon the mode. The calculations for the modes $\mathrm{TE}_{\mathrm{O} 1}$ and $\mathrm{TE}_{02}$ are shown in $\mathrm{Table}$ 6-1.

TABLE 6A-1. Calculation of average power density in a circular waveguide limited by if breakdown at $\mathrm{E}_{0}=2.9 \times 10^{4} \mathrm{~V} / \mathrm{cm}$.

\begin{tabular}{lcc}
\hline Calculation & Mode $\mathrm{TE}_{\mathrm{O}}$ & Mode $\mathrm{TE}_{02}$ \\
\hline $\mathrm{kb}$ & 3.83 & 7.016 \\
$\mathrm{~J}_{0}(\mathrm{~kb})$ & 0.4028 & 0.3001 \\
$\mathrm{~J}_{1}(\mathrm{kr})_{\max }$ & 0.582 & 0.582 \\
$\mathrm{P}_{\mathrm{z}} / \pi \mathrm{b}^{2}, \mathrm{kH} / \mathrm{cm}^{2}$ & 534 & 297 \\
\hline
\end{tabular}


REFERENCES

6-1. M. Porkolab, "Review of RF Heating" from Theory of Magnetically Confined Plasmas, Proceedings of the Course, International School of Plasma Physics, Varenna, Italy (1977), (Pergamon Press, New York, 1979).

6-2. B. W. Reed, 0. N. Bowen, H. M. Hi11, J. Q. Lawson, W. G. Newnan, and A. J. Sivo, Preliminary Report on the Development of RF Auxiliary Heating Systems for TERP-1, Princeton Plasma Physics Laboratory, Princeton University, Princeton, N.J., PPPL-1410 (1977).

6-3. M. Porkolab, "Electron Cyclotron Resonance Heating of Tandem Mirrors," Lawrence Livermore Laboratory, Livermore, CA, UCRL-52634 (1978).

6-4. C. L. Hedrick, R. A. Dand1, J. A. Cobble, R. A. Dory, H. O. Eason, E. G. Harris, G. R. Haste, H. Ikegami, E. F. Jaeger, N. H. Lazar, D. H. McNeill, D. G. McAlees, D. B. Nelson, L. W. Owen, D. A. Spong, and N. A. Uckan, "Transport and Scaling in the Elmo Bumpy Torus (EBT)," Physics and Controlled Nuclear Fusion Research 1976, Sixth Conference Proceedings, Berchtesgaden, Nuclear Fusion Supplement 1977, Vol. II, p. 145 (1977).

6-5. R. S. Symons and H. R. Jory, "Small-Signal Theory of Gyrotrons and Gyroklystons," Proc. Beventh Symposium on the Engineering Problems of Fusion Research, Knoxvilie, TN (1977), (IEEE, New York, 1977).

6-6. V. V. Alikaev, V. A. Flyagin, V. I. Khizhnyak, A. G. Luchinin, G. S. Nusinovich, V. G. Usov, and S. N. Vlasov, "Gyrotrons for the Electron-Cyclotron Plasma Heating in Large Tokamaks," Proc. of Joint Varenna-Grenoble International Symposium on Heating in Toroidal Plasmas, Grenoble, France (1978), (French Atomic Energy Commission, Grenoble, France, 1978).

6A-1. H. E. M. Barlow, "The Relative Power-Carrying Capacity of High-Frequency Waveguides," Proc. IEE (London, Pt. III, Vol. 99, p. 21, 1952). 


\section{CHAPTER 7. PLASMA DIRECT CONVERTER}

$$
\text { W. L. Barr }
$$

Introduction . . . . . . . . . . . . . 165

Particle Energies at Plasma Direct Converter . . . 167

Spacings in Plasma Direct Converter . . . . . . 168

Capacitively Stored Energy ............ . 171

Grid Voltage . . . . . . . . . . . . . 171

Structural Design ............... . 172

Vacuum Requirement ............... 175

Efficiency .................... 178

References .................. . 180 
INT RODUCTION

The single-stage plasma direct converter (PDC) on only one end of the reactor receives $180 \mathrm{MW}$ of ion power and produces $115 \mathrm{MW}$ of electricity for a net efficiency of $64 \%$. The largest single loss of efficiency is due to the high average energy of the alphas $(2.5 \mathrm{MeV})$ at the PDC. Without them the efficiency would be $77 \%$. The PDC has a single collector stage and two watercooled grids, and its maximum diameter is $\sim 13 \mathrm{~m}$. The reason it can be so compact is that only an insignificant amount of electron power has to be handled by the PDC. By selectively leaking most fast ions out one end and most hot electrons out the other, the power loading on the PDC grids is greatly reduced. A simple thermal dump at the electron end receives the $160 \mathrm{MW}$ of electrons plus $45 \mathrm{MW}$ of alphas that cannot be selectively leaked to the PDC. One novel feature of the TMR concept is that the end-loss ions can be preferentially lost out one end and the electrons preferentially lost out the other end. This condition is accomplished by lowering the potential in one plug slightly so that, as the ions that are confined in the central cell scatter in phase space, they escape over the lower potential barrier before they can gain enough energy to pass over the higher one. The electrons are unaffected by the change in one plug potential since they are confined by the combination of the positive potential, $\phi_{e}$, and the magnetic mirror. By holding the entrance grid to the ion collector at a low negative potential and by decreasing the magnetic mirror on the electron end, the central cell electrons can be allowed to leak out one end before they scatter into the loss cone for the ion end.

The great advantage in separating the end-loss electrons from the ions is gained at the ion end. At the PDC, which recovers most of the ion power 
electrically, an electron repeller grid (see Fig. 7-1) prevents electrons from reaching the ion collector, which is held at high positive potential. Any electrons flowing out of or produced in the ion end are reflected by the repeller grid and are eventually collected on the entrance grid, which immediately precedes the repeller grid. The entrance grid therefore has to handle the heat generated not only by the small fraction of the ions that are intercepted but also by all of the electrons that go out that end. By causing most of the end-1oss electrons to go out the other end, the heat load on the entrance grid is drastically reduced-in this case from over $160 \mathrm{MW}$ to essentially only the intercepted fraction of the $155 \mathrm{MW}$ of ions.

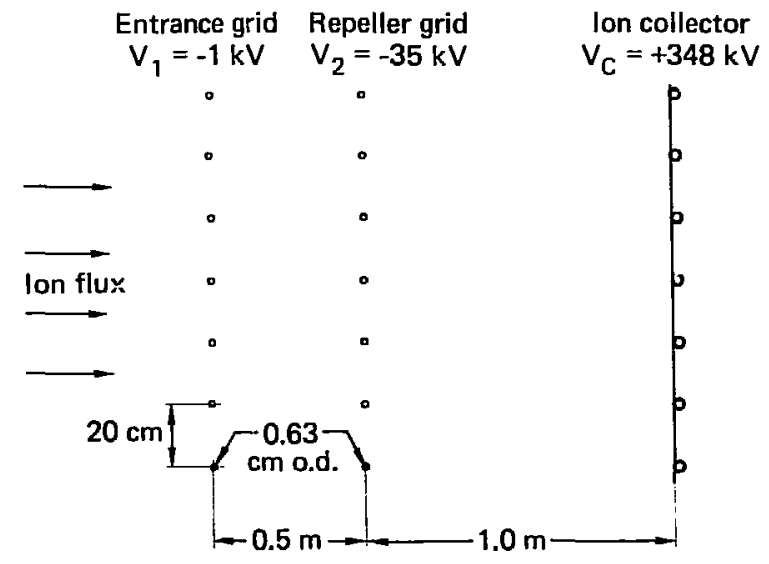

Fig. 7-1. Grids and collector in plasma direct converter.

The low heat load allows a relatively compact PDC for the ion power. The small size of the PDC allows the use of convectively cooled grids, which in turn allow a higher power density than radiative cooling allows. The longest grid element is about $5 \mathrm{~m}$ long, and the diameter could be as large as $1 \mathrm{~cm}$. Grids of this size can be water-cooled and can easily handle more than 
a $300-\mathrm{W} / \mathrm{cm}^{2}$ heat load on one side. The flux tube containing the escaping ions must have a cross-sectional area of $65 \mathrm{~m}^{2}$ to reduce the average power density to about $280 \mathrm{~W} / \mathrm{cm}^{2}$. Because of the circularizing coil the $\mathrm{flux}$ tube has a circular cross section, and the field liner radiate out to form an expanding cone. Therefore, the grids and collector of the PDS should each be formed into the curved surface of a spherical segment in order to intercept the ions at nearly normal incidence.

PARTICLE ENERGIES AT PLASMA DIRECT CONVERTER

The current of lost fuel ions going to the PDC is just equal to the total number of fuel ions confined in the central ce11 at any one time divided by the mean confinement time:

$$
I_{c}=\frac{q n_{c}^{2} v_{c}}{\left(n_{\tau}\right)_{c}}=333 \mathrm{~A}
$$

On the average each ion escapes with a mean energy of $\mathrm{T}_{\mathrm{ic}}=40 \mathrm{keV}$ (i.e., thermal energy in two degrees of freedom), and is then accelerated by the potential difference $\phi_{c}+\phi_{e}=355 \mathrm{kV}$ before reaching the entrance grid at the PDC. The average energy is therefore $395 \mathrm{keV}$, and the minimum is $355 \mathrm{keV}$. Ions that escape after being confined in a plug also have at least $355 \mathrm{keV}$ of energy at the PDC, but their mean energy is $755 \mathrm{keV}$. The current of plug ions to the PDC is only

$$
I_{p}=\frac{q n_{p}^{2} v_{p}}{(n \tau)_{p}} \times 2=4.8 \mathrm{~A} .
$$


The alpha particles that result from the fusion reactions arrive in the end tanks in three different energy groups. Because the effective magnetic mirror ratio seen by the particles in the centraî cell is only $3.65,15 \%$ (50 MW) of the alphas are born in the loss cone and escape immediately with their full $3.52 \mathrm{MeV}$ of energy. Half of the resulting $28.6 \mathrm{~A}$ (electrical) goes out each end and, after acceleration by the difference in potential $\left(\phi_{e}=200 \mathrm{kv}\right)$, the alphas arrive with $1960 \mathrm{keV}$ per unit of charge. The trapped $85 \%$ of the alphas transfer $129 \mathrm{MW}$ to the plasma electrons and $136 \mathrm{MW}$ to the ions. While cooling by transferring energy to the plasma, $22 \%$ (42 A) of the alphas scatter into the loss cone and escape with $20 \mathrm{MW}$ of the remaining $25 \mathrm{MW}$ of power. They, too, are accelerated by $\phi_{e}$ and arrive half at each end, with a mean energy of $783 \mathrm{keV}$. The other $63 \%$ of the alphas thermalize with the central plasma and eventually diffuse radially, depositing 5 MW on the wall. They are reionized as they leave the wall, and stream out along the outer magnetic field lines as cold ions. The result is that the alphas deliver $44.8 \mathrm{MW}$ out each end, since they are too energetic to be affected by the difference in potential at the two plugs.

Except for the alphas, the total ion energy distribution at the PDC is narrow, with a high minimum of $355 \mathrm{keV}$ per unit of charge. This is an ideal distribution for a single-stage PDC.

SPACINGS IN PLASMA DIRECT CONVERTER

The grid parameters are determined by the spacing d between the collector surface and the surface of the negative grid (see Fig. 7-1). Elementto-element spacing in a grid must be small compared to d to give reasonably smooth surfaces of equipotential near the grid so that the ions are not 
deflected by more than about $10^{\circ}$. The spacing $d$ is determined by the current density of the ions. The current density $j_{i}$ is about:

$$
j_{i} \approx \frac{340 \mathrm{~A}}{65 \mathrm{~m}^{2}}=5.2 \mathrm{~A} / \mathrm{m}^{2},
$$

if we ignore the alphas since their contribution to space charge is small. At a given current density, the space charge of the ions seriously distorts the applied electric field near the collector if the spacing $d$ is too large. For spacings larger than a maximum value, a virtual anode forms in front of the collector. The location of this virtual anode is the maximum effective spacing, $d_{\max }$, and is given approximately by Child's law for the space charge limit in a plane diode. When solved for $d_{m a x}^{2}$, Child's law states that:

$$
d_{\max }^{2}=\frac{4 \varepsilon_{0}}{9} \sqrt{\frac{2 q}{M}} \frac{v^{3 / 2}}{j_{i}},
$$

where $\varepsilon_{0}=8.85 \times 10^{-12} \mathrm{~F} / \mathrm{m}, q$ is the charge, $M$ is the mass of an ion, and $V$ is the voltage difference across the space $d_{\max }$. In this expression it is assumed that the ions all have zero energy at the anode. In the PDC the collector voltage is about $350 \mathrm{kV}$ and only some of the ions are decelerated to near zero energy. The average ion arrives with $40 \mathrm{keV}$ of kinetic energy remaining. For this reason, Child's law gives a slightly smaller value for $d_{\max }$ than the true value. Actually, in a PDC there is no serious consequence of exceeding $d_{\max }$. If the spacing is greater than $d_{\max }$, a virtual anode forms at $d_{\text {max }}$ as the space charge of the ions increases the potential there until it is essentially equal to anode (i.e., collector) potential. If the potential at the virtual anode were to rise above the collector voltage, some ions would be reflected before reaching the collector. But, the emission of secondary electrons from the collector prevents the space charge potential from rising more than a few volts above that of the collector. The result is 
that in a PDC the effective spacing adjusts automatically so that it satisfies Childs's law.

The voltage difference across the spacing $d$ is approximately the difference between the $350-\mathrm{kV}$ collector voltage and the $-35-\mathrm{kV}$ grid voltage, or $385 \mathrm{kV}$. Child's law then gives $\mathrm{d}_{\max }=1.58 \mathrm{~m}$ for an equal mixture of $350-\mathrm{keV}$ tritons and deuterons making up the current density $j_{i}=5.2 \mathrm{~A} / \mathrm{m}^{2}$. We choose $\mathrm{d}=1.0 \mathrm{~m}$ and set the spacing between the negative grid and the grounded grid equal to $0.5 \mathrm{~m}$.

The longest grid elements are approximately 5 m long. By using highstrength, high-temperature alloys, such as Ta-10 $\mathrm{W}$ or Mo-0.5 Ti (which can stand an internal water pressure of 400 bars), the $280-W / \mathrm{cm}^{2}$ heat 1 oad could be handled on grid elements less than $2 \mathrm{~mm}$ in outer diameter. Elements of that small size would intercept only about $1 \%$ of the ions. But, the manifolds to supply the high pressure water would intercept much more. Also, there is a danger from the possible failure of such a high pressure system.

For the present study we consider larger diameter elements that can handle the $280 \mathrm{~W} / \mathrm{cm}^{2}$ on one side with much lower water pressure and temperature. The elements considered are tubes of 316 stainless steel with a $0.63-\mathrm{cm}$ outside diameter and 1-ma-thick walls. An input water pressure of 20 bars and an outlet pressure of 5 bars allow a temperature rise in the water of $120^{\circ} \mathrm{C}$ in a 5-m-long tube. The maximum tube wall outer temperature is $275^{\circ} \mathrm{C}$. The spacing "a" between tubes in a grid should be small compared with $\mathrm{d}=1000 \mathrm{~mm}$, but much larger than the 6.3-mm-diam of the tubes. We choose $a=200 \mathrm{~mm}$, making the grid $3.2 \%$ opaque to the ions. The water manifolds and the support structure add to this opacity. 


\section{CAPACITIVELY STORED ENERGY}

In designing the support structure, the total capacitively stored electrical energy must be considered. The stored energy that can be released in a spark must be kept below about $10 \mathrm{~J}$ to prevent damage to the electrodes. To I imit the stored energy available, the PDC must be electrically divided into sections. The total stored energy $E_{s t}$ in the $P D C$ is:

$$
\mathrm{E}_{\mathrm{st}}=\frac{1}{2} \mathrm{Cv^{2 }}
$$

where

$$
\begin{aligned}
& C=\frac{E_{0} A}{d} \\
& \epsilon_{0}=8.85 \times 10^{-12} \mathrm{~F} / \mathrm{m} .
\end{aligned}
$$

$A=65 \mathrm{~m}^{2}$ is the surface area of the $\mathrm{PDC}, \mathrm{d}=1.0 \mathrm{~m}$ is the grid-to-collector spacing, and $v=385 \mathrm{kV}$ is the potential difference between grid and collector. The above gives $E_{s t}=43 \mathrm{~J}$, indicating that the $P D C$ must be divided into at least five sections. We choose the configuration shown in Fig. 7-2, which divides it into seven sections and allows for the possibility of higher power density and higher ion energy near the center.

\section{GRID VOLTAGE}

Each section of the PDC is similar to a plane, triode vacuum tube. We must operate it beyond the cutoff condition to prevent electrons at the entrance gri.1 (cathode) from reaching the collector (plate). Spangenburg ${ }^{7-1}$ 


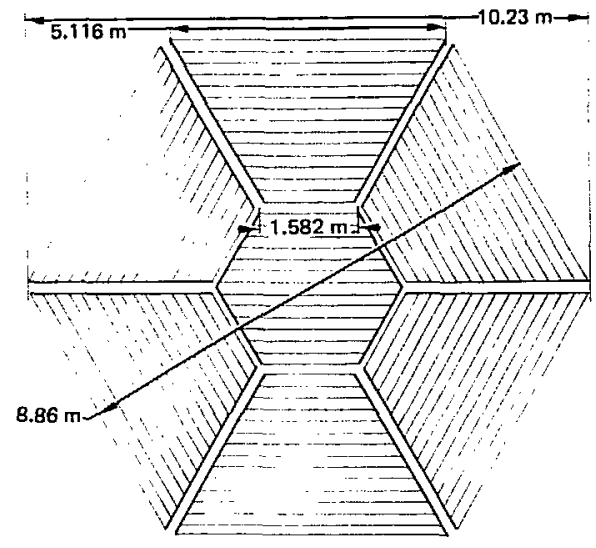

Fig. 7-2. Seven sections of entrance grid.

has analyzed the grid voltage required for cutoff in triodes, and his graph shows that the grid voltage, $v_{g}$, must satisfy:

$$
\mathrm{v}_{\mathrm{g}} \leq-\mathrm{v}_{\mathrm{p}} / 13
$$

for our geometry ratios. Here, $v_{p}=350 \mathrm{kV}$ is the collector voltage. Spangenburg's analysis does not include the space charge of the ions. The effect of space charge can be approximately allowed for by observing that in a space-charge 1 imited diode (considering only the negative grid and the collector now), the electric field at the cathode is just $4 / 3$ times the vacuum field. We can include this effect by reading Spangenburg's graph at $3 / 4$ of the actual d value. This gives $\mathrm{v}_{\mathrm{g}} \leq-\mathrm{v}_{\mathrm{p}} / 10=-35 \mathrm{kV}$. We set $\mathrm{v}_{\mathrm{g}}=-35 \mathrm{kV}$, since the PDC is well below its space charge limit.

STRUCTURAL DES IGN

The hexagonal shape of the PDC shown in Fig. 7-2 offers a practical approximation to the ideal spherical surface. The six sections outside the central hexagon are tilted toward the incident beam to receive it at nearly 
normal incidence. The $6.5-\mathrm{m}^{2}$ area of the central section is smaller than the $11.1 \mathrm{~m}^{2}$ of each outer section because of the higher power density at the center and because of the unused outer corners. In the center section the longest grid tube is $3.16 \mathrm{~m}$ as compared to $5.12 \mathrm{~m}$ for the longest outer tube. This difference allows the center section to receive up to twice the power density that the outer tubes are designed for.

There are 17 tubes in each outer grid section and they vary in length from $1.58 \mathrm{~m}$ to $5.12 \mathrm{~m}$. The center section has 14 tubes with lengths from 1.58 to $3.16 \mathrm{~m}$. It is assumed here that all tubes have the same inner and the same outer diameters. A more detailed design should adjust the diameters so that the temperature rise of the water is the same in all tubes. Similarly, the cantilever supports for the outer sections which also serve as water manifolds (see Fig. 7-3) could be optimized by varying the inner and outer diameters along the length. In this way the sag could be limited with a minimum of external area exposed to the ion flux. Here we simply note that the cantilever supports for the outer sections could be made from 316 stainless ste:1 tubes with uniform 25-mm 0.d. and 1.3-m wall thickness. The greatest sag would be 0.23 m at the tip of the supports for those outer grid panels that lie in nearly vertical planes. This greatest sag would occur in the plane of the grid. The center section is supported from the rear (see Fig. 7-4), and we assume that the same size tubes can be used there.

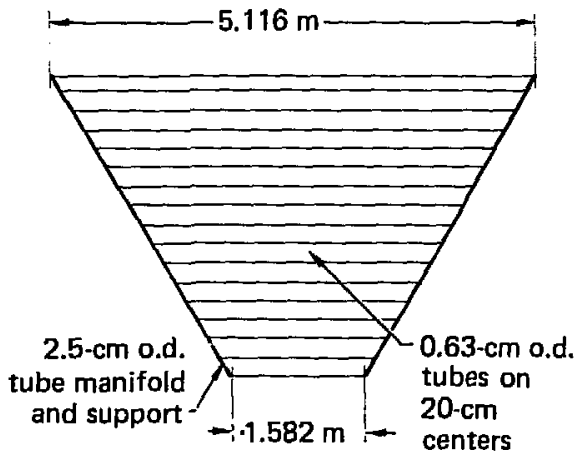

Fig. 7-3. Outer section of entrance grid. 


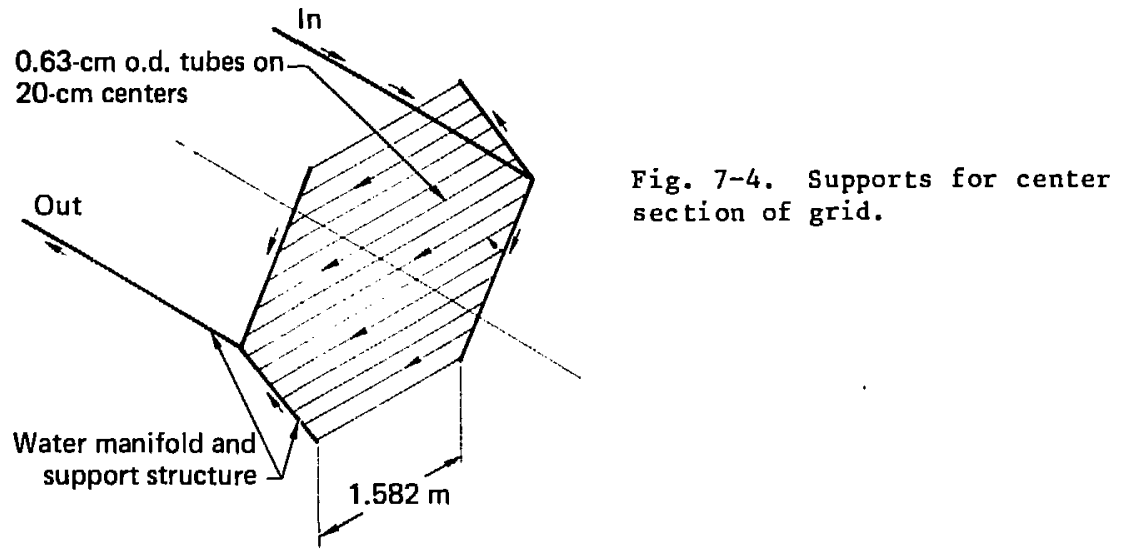

There are six outer panels for each grid, and each panel requires two support tubes $3.76 \mathrm{~m}$ in length. But only $3.32 \mathrm{~m}$ of that length is exposed to the flux of ions. The center panel of each grid requires support tubes on four of the six sides. Each side is $1.58 \mathrm{~m}$ long. Therefore, the total length of exposed support tubes in each complete grid is $46.2 \mathrm{~m}$ giving an intercepting area of $1.15 \mathrm{~m}^{2}$. There is a $6.3-\mathrm{mm}$ grid element every $200 \mathrm{~mm}$ across the grid, giving an intercepting area of $2.05 \mathrm{~m}^{2}$ for the elements. The total intercepting area is $3.2 \mathrm{~m}^{2}$, or $4.9 \%$ of the $65 \mathrm{~m}^{2}$ total. Both grids have identical structures.

The center section of each grid is supported from behind by two 25-mm stainless steel tubes, which also carry the cooling water. High voltage insulators are required where the four tubes pass through the collector surface. The insulators must hold off 350 and $385 \mathrm{kV}$ at the supports for the entrance and the negative grid, respectively. Shielding against sputtering must also be provided at each insulator. Because of the 20-cm spaces between grid elements, no insulation is needed where the supports for the entrance grid pass near the negative grid. Only a negligible fraction of the collector surface area need be lost to the feedthrough insulators. If each insulator 
structure has an outer diameter of $15 \mathrm{~cm}(\sim 50 \mathrm{kV} / \mathrm{cm})$, th.e tolal area lost for ion collection is only $0.07 \mathrm{~m}^{2}$. Figure $1-6$ shows a sketch of the structure.

VACUUM REQUIREMENT

The vacuum in the region between the plug and the PDC must be good enough so that only an allowable fraction of the escaping ions undergo charge exchange. Any ion that does capture an electron from a gas molecule will arrive at the PDC as a fast neutral. It will impact the collector with its full $\sim 355 \mathrm{keV}$ of energy and will generate heat but no direct electric power. The cold ion that is produced is trapped by the combination of the magnetic field and the high positive potentials of the plug on one side and the PDC on the other. It will finally diffuse to a magnetic flux tube that intercepts a grid element and will be collected there. Since itr energy is low, very little heat is generated, but electrical power is consumed at the negative grid.

It is more serious if the ion suffers charge exchange inside the PDC near the collector where the potential is positive. Then the resulting ion is accelerated and can deposit a significant amount of heat on the grid that collects it.

The cross section for charge exchange of fast $\mathrm{D}^{+}$with $\mathrm{D}_{2}$ molecules is $4 \times 10^{-18} \mathrm{~cm}^{-2}$ at $395 \mathrm{keV}$ and has a broad maximum value of $8.6 \times 10^{-16} \mathrm{~cm}^{-2}$ between about 4 and $20 \mathrm{keV}$. Those ions that reach the PDC with only slightly more than the minimum $355 \mathrm{keV}$ are decelerated into that 4- to 20-keV range where the cross section is large. Ions with the average $395 \mathrm{keV}$ or more see a much smaller average cross sectici. We can, therefore, estimate the vacuum requirement by calculating the pressure at which about $2 \%$ of those worst-case (355-keV) ions undergo charge exchange. The fraction, $F_{0, L}$, of those ions 
that capture an electron in a charge-exchange collision in going the distance L from the plug to the coilector surface is:

$$
\mathrm{F}_{0, \mathrm{~L}}=1-\exp \left\{-\int_{0}^{\mathrm{L}} \mathrm{n}_{0} \sigma_{10} \mathrm{dx}\right\},
$$

or approximately

$$
\mathrm{F}_{0, \mathrm{~L}} \approx \int_{0}^{\mathrm{L}} \mathrm{n}_{0} \sigma_{10} \mathrm{dx}
$$

Here, $n_{0}$ is the neutral gas density, $\sigma_{10}$ is the cross section for electron capture, and both $\mathrm{n}_{0}$ and $\sigma_{10}$ may vary with the distance $\mathrm{x}$ along the ion path. For those worst-case ions, the last $20 \mathrm{~cm}$ before collection dominates the integral so that:

$$
\mathrm{F}_{0, \mathrm{~L}} \approx \mathrm{n}_{0} \sigma_{10}(\max )(20 \mathrm{~cm})
$$

For $\mathrm{F}_{0, \mathrm{~L}} \leq 0.02$, we must have $\mathrm{n}_{0} \leq 1 \times 10^{12} \mathrm{~cm}^{-3}$ giving a pressure $p \leq 3 \times 10^{-5}$ Torr. This is a rough estimate of the vacuum requirement. For a more accurate determination of the allowed neutral density, a proper average of $\mathrm{F}_{0, \mathrm{~L}}$ must be calculated using the ion energy distribution.

The allowed pressure, $P$, and the gas throughput, $Q$, then determine the pumping speed, s, that is required. The 340-A input of atomic ions becomes $Q=30$ Torr-litres/s of molecular gas. Then:

$$
\mathrm{S}=\frac{\mathrm{Q}}{\mathrm{P}}=\frac{30 \text { Torr-1itres } / \mathrm{s}}{3 \times 10^{-5} \text { Torr }}=1.0 \times 10^{6} \mathrm{litres} / \mathrm{s}
$$

is the required pumping speed. Cryopumping can be used not only for the hydrogen isotopes, but also for the small amount of He. The He will be pumped by cryotrapping because of the 30-times-greater input of $\mathrm{D}_{2}$ and $\mathrm{T}_{2}$ which will act as the sorptive medium. A cryopump with two sets of chevrons has a specific pumping speed of 4.41 itres $/ \mathrm{s}-\mathrm{cm}^{2}$ for a mixture of $\mathrm{D}_{2}$ and $\mathrm{T}_{2}$ gas at 
$300 \mathrm{~K}$. Therefore, only $23 \mathrm{~m}^{2}$ of cryopanels is needed. As compared to the $65-\mathrm{m}^{2}$ frontal area of the PDC, the $23 \mathrm{~m}^{2}$ of cryopanels seems rather modest. The cryopanels can easily be located on the side walls in the PDC tank where they are protected from neutron bombardment. Figure 7-5 indicates their location.

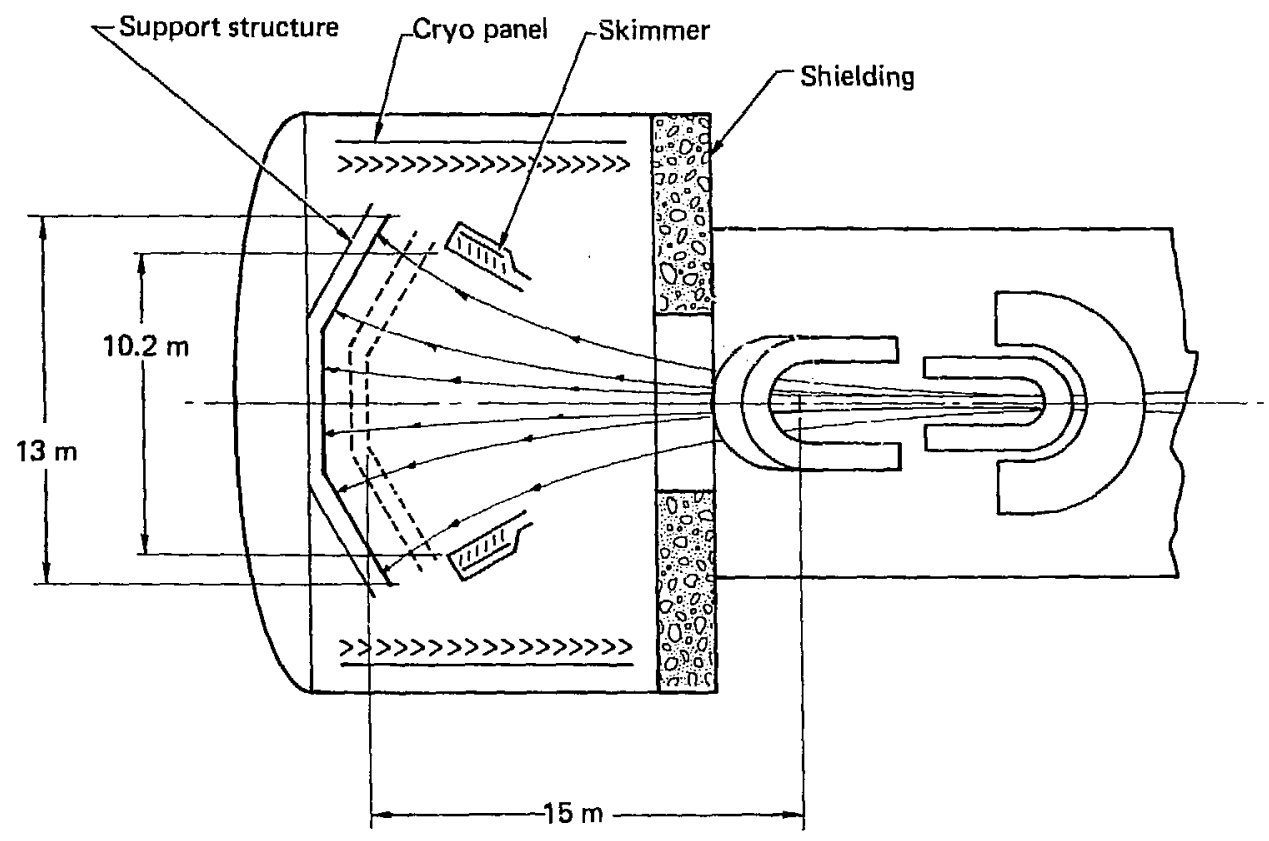

Fig. 7-5. Location of cryopanels and skimmers.

Skimmers near the PDC remove the cold plasma that streams out along magnetic field lines that lead from the outer boundary of the central cell plasma. These skimmers (see Fig. 7-5) direct their gas onto enclosed cyropanels. 


\section{EFFICIENCY}

The efficiency of the PDC can best be determined by considering the losses. The losses are:

- Electrons. We assume here that all hot electrons are lost out one end and do not affect the PDC at the other end. The cold electrons that neutralize the space charge of the ions give rise to only an insignificant loss.

- Grid interception. The grids together wich their supfurts each intercept $4.9 \%$ of the ion flux giving a $9.8 \%$ total loss.

- Repeller grid power consurption. The negative grid is held at $v_{g}=-35 \mathrm{kV}$ by a power supply that must supply not only the $4.9 \%$ of $360 \mathrm{~A}$ of ions that it intercepts, but also about three secondary electrons for each ion intercepted. The above amounts to a power loss of about $17 \mathrm{~A}$ of ions and $50 \mathrm{~A}$ of electrons at $35 \mathrm{kV}$, or $2.4 \mathrm{MW}$, which amounts to $1.5 \%$ of the incident $159 \mathrm{MW}$ of total ion power.

- Energy spread. The ideal collector potential is $355 \mathrm{kV}$, the maximum value before reflecting even those ions with the lowest energy. The average ion, including alphas and plug ions, has $482 \mathrm{keV}$ of energy per unit of charge at the PDC and impacts the ideal collector with $127 \mathrm{keV}$, giving a $10 \mathrm{ss}$ of $26 \%$ in directly recovered power.

- Deflection at the grids. Those ions that pass close to a grid element, especially in the negative grid, suffer some deflection. The deflection is small because of the high energy of the ions, and is estimated to be less than $8^{\circ}$ maximum. After an $8^{\circ}$ deflection, a minimum-energy (355 kev) ion has only $348 \mathrm{keV}$ of energy in the forward direction. For this reason the collector voltage must be reduced from 355 to $348 \mathrm{kV}$ to prevent the reflection of some ions. This gives a $2 \%$ loss of recovered power. 
- Residual magnetic field. The strength of the magnetic field at the PDC is less than that in the central cell in inverse proportion to the areas. This gives $0.075 \mathrm{~T}$ at the $\mathrm{PDC}$. Since the ions were confined electrostatically and not magnetically, this residual magnetic field does not limit the parallel energy at the PDC, and causes no loss of recovered power.

- Charge exchange. By maintaining the pressure in the PDC vacuum tank below $3 \times 10^{-5}$ Torr, the loss of fast ions by charge exchange can be made negligible. The power consumed by the collection of the resulting cold ions on the repeller grid is then also negligible.

- Coolant pumping power. Because of the rather large size of the tubes that make up the grids and those that can be used to cool the collector, we assume the pumping power is negligible.

After allowing for all of these various losses, a total of $338 \mathrm{~A}$ of ions are collected at $348 \mathrm{kV}$, giving $115 \mathrm{MW}$ of net electric power output from the PDC after supplying $2.4 \mathrm{MW}$ to the repeller gria, yielding an efficiency of $64 \%$. The largest single loss is that due to the high average energy $(\sim 2.5 \mathrm{MeV})$ of the alphas at the PDC. The efficiency is $77 \%$ if the alphas are not included in the calculation. 


\section{REFERENCES}

7-1. K. R. Spangenburg, Vacuum Tubes (McGraw-Hill Book Co., New York, 1948), P. 125 ff. 


\title{
CHAPTER 8. CENTRAL CELL
}

W. S. Neef, Jr.

\begin{abstract}
Centra1 Ce11 Design . . . . . . . . . . 183
Cylindrical Pods . . . . . . . . . . . . 184

Blanket and Shield Neutronics ......... 191

Blanket and Shield Assembly ........... 193

Shield ................ . 196

Seal Washer . . . . . . . . . . . 196

Central Cell Servicing . . . . . . . . . . 198

Large Module Replacement . . . . . . . . 198

Pod-Module Replacement . . . . . . . . 201

Summary of Central Cell Design and Cost Estimate . . 204

References .................. 206
\end{abstract}


CENTRAL CELL DESIGN

The heart of any fusion power plant is the energy conversion blanket which absorbs thermonuclear neutrons (converting the neutron kinetic energy to temperature rise in a circulating coolant), and breeds tritium as future fucl. The TMR blanket and shield assembly has the simple geometry of a straight cylindrical annulus.

Our first attempt at a TMR blanket design ${ }^{8-1}$ used wedge-shaped pressure-tight submodules arranged in a ring. They contained quasi-static lithium in canisters that were ccoled by helium at a pressure of 60 atm and a maximum coolant temperature of $550^{\circ} \mathrm{C}$. The shape of these modules and our desire to avoid cold-welding by contact led us to a rigid pressure container of nonideal shape and a large structural fraction (about 17\%' Sufficient tritium breeding was achieved but the design proved expensive. Contributing to the high cost of the central cell was a low solenoid current density $\left(1200 \mathrm{~A} / \mathrm{cm}^{2}\right)$, which we are now confident can be tripled with present niobium-titanium superconductor. The MFTF yin-yang coil is designed for $2980 \mathrm{~A} / \mathrm{cm}^{2}$ with a $7.5-\mathrm{T}$ field at the conductor. Our conductor fiald strength will be about half that value.

Improvements in tandem mirror physics leading to higher reactor $Q$ have been under development. Preliminary results indicate that best reactor econorics will probably be at a first-wall neutron power loading between 2 and $4 \mathrm{NW} \cdot \mathrm{m}^{-2}$. We have proceeded to develop a new blanket and shield concept for the rentral cell quite independent of the end-plug changes, knowing that wh could deal with the transition zone near each plug without basically changing a new centra1-ce11 design approach. 
In order to reduce reactor cost and to improve maintainability we have established the following set of design goals for this iteration of the TMR design. They are:

- Simple design; easily built parts

- Rugged, reliable, pretested components

- Simple building for shielding, containment, and support

- Operation by a standard: If it doesn't require replacement, don't move it

- Remote servicing by simple motions with simple tools

- Inexpensive construction, operation, and maintenance We have achieved these goals by redesign of the central cell.

CYLINDRICAL PODS

It is well known that a cylinder is a much more material-efficient pressure vessel than our previous wedge. Long cylinders were studied (some as long as the entire central cell) but the problem of replacement ended that investigation. Cylinders pointing toward the plasma centerline have been pro. posed, $(8-2)$ but in that case each was removed from inside the vacuum vessel (i.e., toward the plasma). We wished to minimize plumbing disconnects both for first-wal1 replacement and for flaw correction. Further, we wished to propose a reactor offering two modes of blanket change: (1) remove large segments for first-wall change; and (2) remove only the offending blanket submodule in the event of some malfunction, to effect the quickest possible repair.

Our new design employs annular rows of short tubes. Four and five rows were considered, the latter to be used if the tritium breeding material is liquid lithium. Four rows are sufficient if lithium oxide is the breeder. To 
achieve the highest breeding material content the tubes (called "pods" in this design) change in diameter approximately in proportion to distance from the reactor centerline. Figure 8-1 shows an arrangement of four rows of pods. The pods are mounted permanently to two different gas distributor housings. (Figs. 8-2 and 8-3). The two shapes are necessary to permit withdrawal of the pod-modules radially inward from their mounting location on the shield. If one of the smaller pods must be repaired it will be necessary to remove the two neighboring large pods to gain access to the small one. This method of removal can be used for correction of a pod malfunction by moving a servicecar down the bore of the reactor and extracting and replacing the offending unit. This remote servicing machine will be described in more detail in CEntral Cell Servicing.

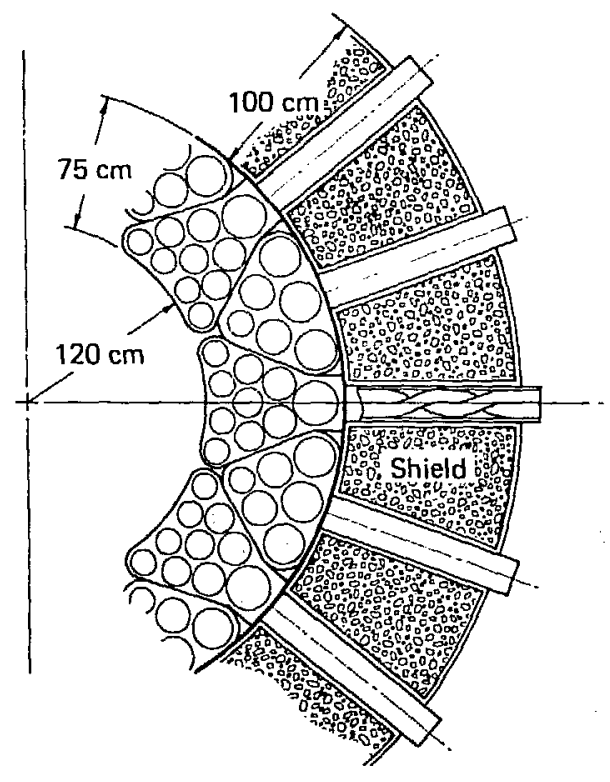

Fig. 8-1. Cross section of blanket and shield. 

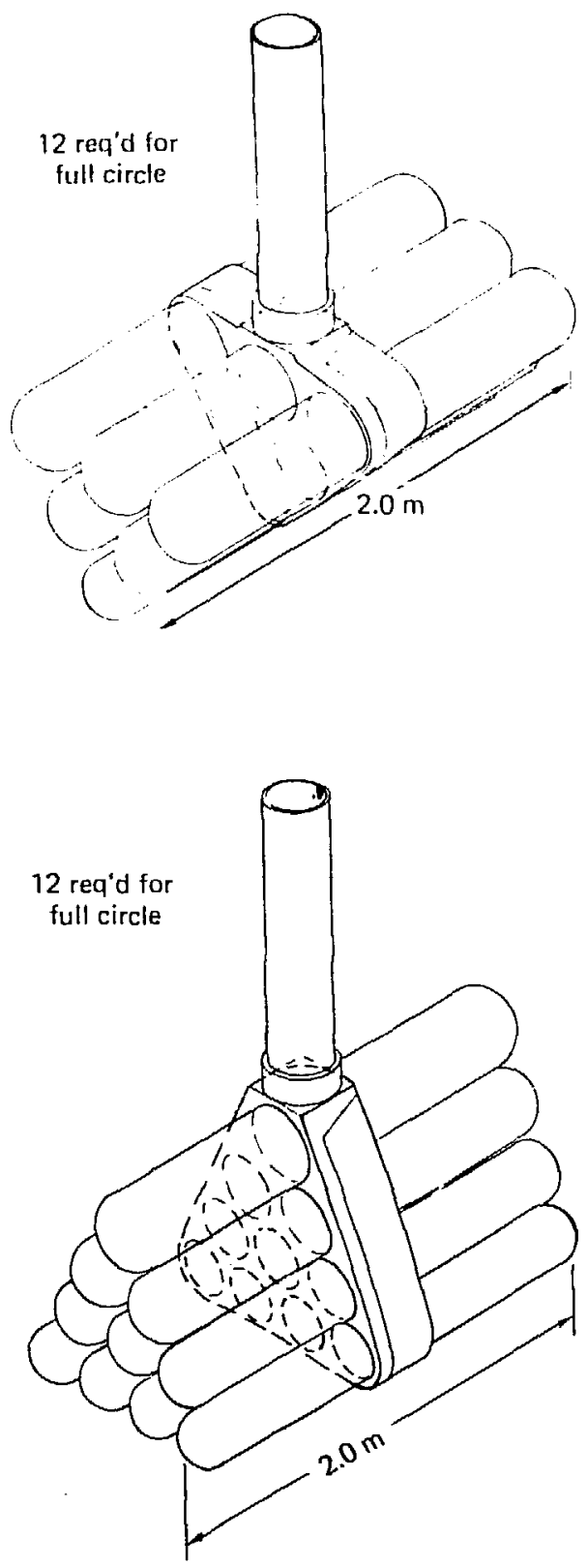

Fig. 8-2. Blanket pod cluster-Type S.

Fig. 8-3. Blanket pod cluster-Type T. 
Each gas-distributor module has a stem that extends through to the back of the shield where it is welded at the time of assembly. Figure 8-4 shows this stem containing gas inlet and exit tubes (twisted to reduce neutron streaming). A bolted cover flange carrying helium coolant tubes provides the gas seal. That flange must be removed to allow access to the aforementioned stem weld. The flange's coolant tubes mate with the twisted gas tubes in the module stem. The seal between those tubes need not be perfect. The flange seal and the tube joints between the cover flange and the supply and return manifolds must be of high-vacuum quality and must be tested using mass spectrometer methods.

Each pod (there are ten in one assembly and six in the other) passes through the gas distributor support. Two metres were chosen as the pod length, which sets the length of a major central cell module at 2 m also. The mass of such a module is approximately 500 tonnes.

The construction features of the pod and its relationship to the gas distributors is shown in Figure 8-5. The canister containing the breeding material, $\mathrm{Li}_{2} \mathrm{O}$ in finely divided granules, is sheet stainless steel with a thin coating nearly impervious to tritium. Berylium oxide is one coating possibility. Tritium is removed from the canister by a small purging flow of helium at the same pressure as the main coolant stream. The fraction of maximum theoretical density that is attainable for $\mathrm{Li}_{2} \mathrm{O}$ is not known; it most probably lies between 60 and $90 \%$. Our calculations of tritium breeding ratio indicate a range of values from 1.07 at $50 \%$ theoretical density to 1.27 at 90\%. The more finely divided particles will allow prompt tritium purge and hence lower blanket inventory. Work by JAERI $8-3,8-4$ in Japan leads us to conclude that tritium can be extracted easily so long as the particles of $\mathrm{Li}_{2} \mathrm{O}$ are small, preferably 10 to $100 \mu \mathrm{m}$ across. 


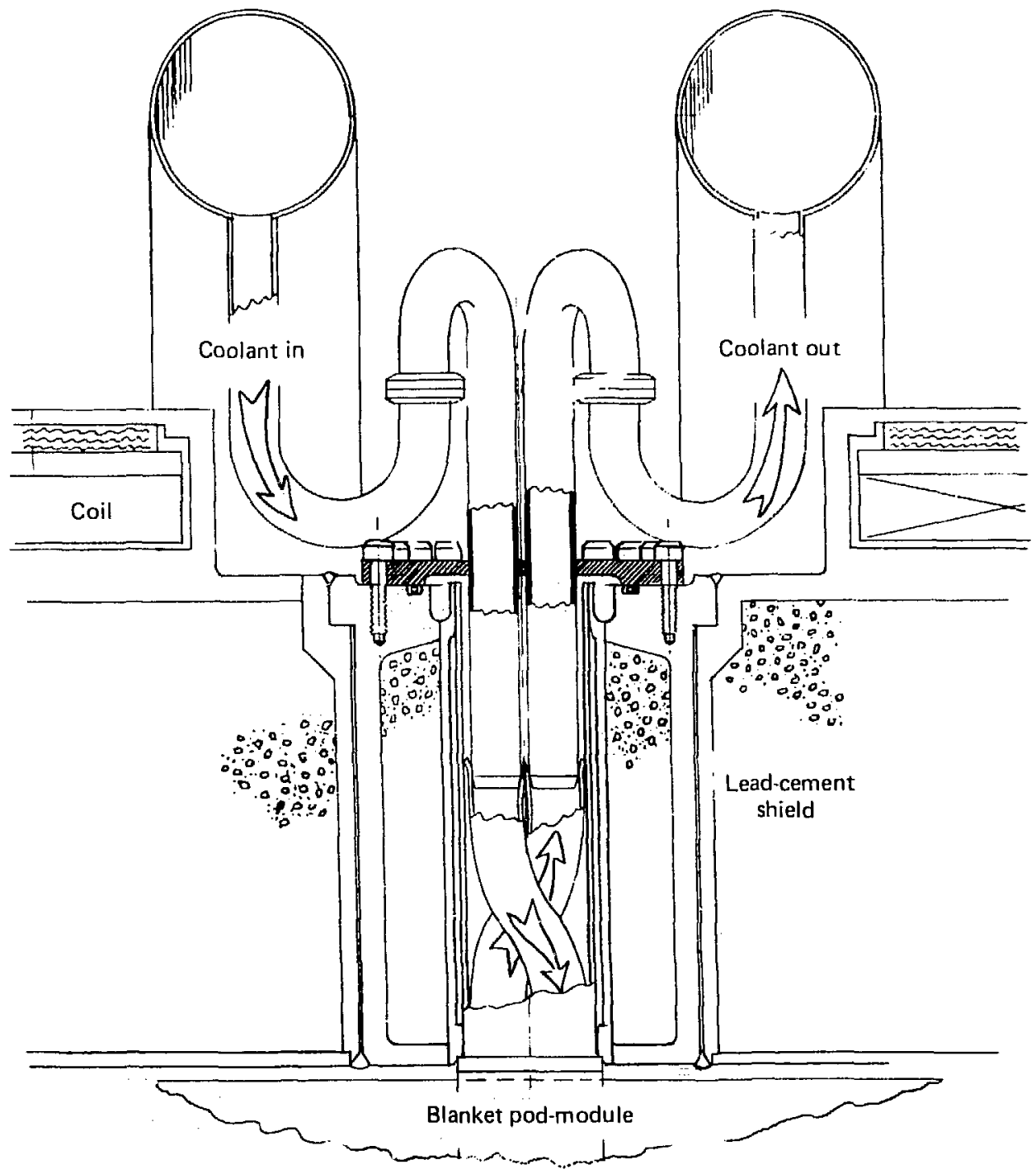

Fig. 8-4. Module coolant connection. 


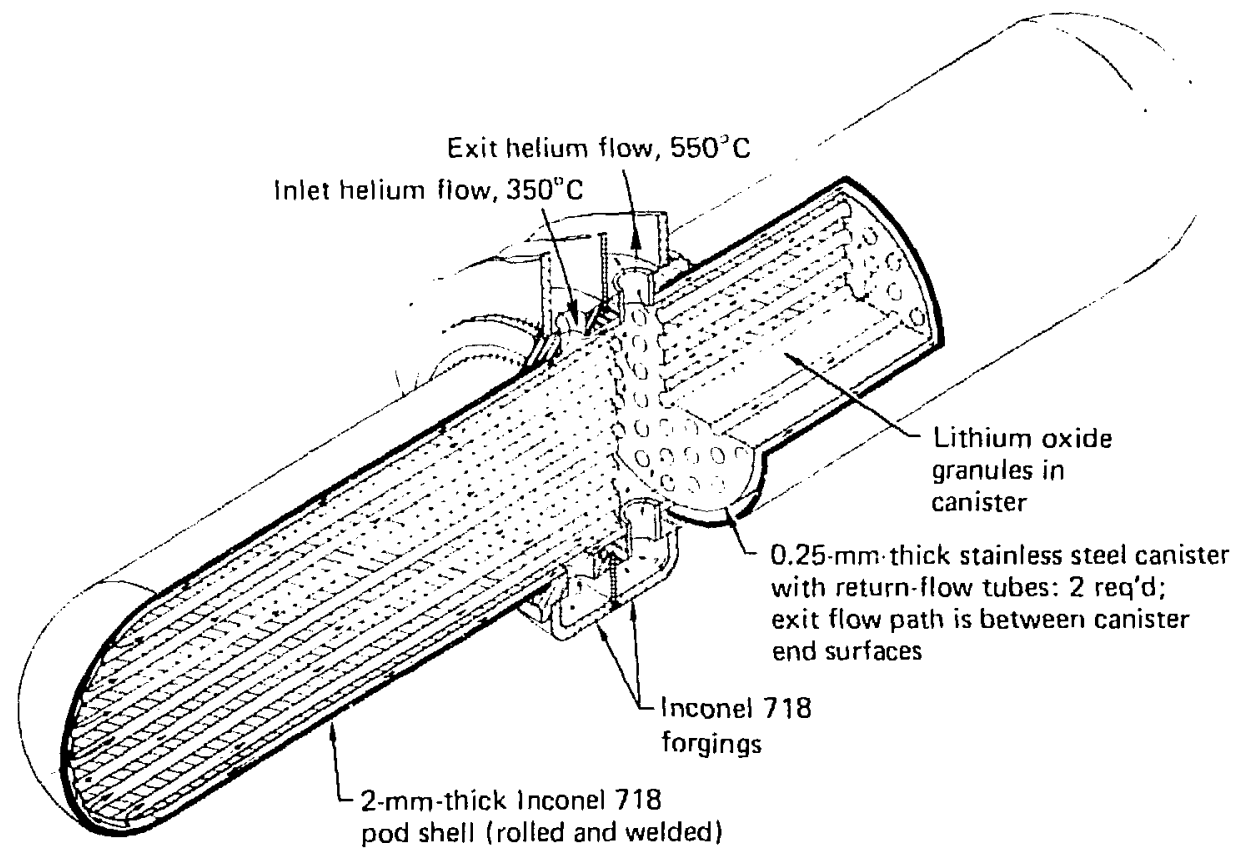

Fig. 8-5. Blanket pod.

The coolant flow path brings helium first to the pod shell where it flows toward the hemishell end. The outside of the $\mathrm{Li}_{2} \mathrm{O}$ canister is cooled simultaneously by that flow stream. Upon reaching the end of the shell the helium returns, entering tube passages running lengthwise through the canister thereby cooling the internal mass of $\mathrm{Ii}_{2} \mathrm{O}$. The presence of the tritiumpurging helium flow at 60 atm pressure is essential to obtain blanket cooling. The helium serves as the heat transfer medium from the $\mathrm{Li}_{2}{ }^{\mathrm{O}}$ particles to the tube walls of the canister. The number and location of these $0.5-\mathrm{cm}^{-}$ diam tubes has only been estimated. 
To return the coolant to the gas distributor it is necessary that a collection gap about $4 \mathrm{~cm}$ long be provided just adjacent to the midplane of the canister. Helium flows radially outward from this collector plenum through short tubes that also serve to anchor the canister within the pod. To further stabilize the canister position in the flow stream, several fins or corrugations of the canister outer shell will serve to pilot it inside the pod.

It will be noted that the outer shell of the canister of $\mathrm{Li}_{2} \mathrm{O}$ is continuous through the gas distributor. There are two reasons: the breeding of tritium requires some lithium in that zone in order to achieve an adequate breeding ratio, and also, structural members are required to 1 imit the bending stress in the gas distributor face-plate. The two halves of the pod shell are joined by threading, which permits a clamping grip on the inlet/exit helium flow divider. Figure 8-6 shows an exploded view of the gas distributor. Once the two pod shells are threaded, the pod collars can be brazed to the gasdistributor housing. This blazing locks the whole gas-distributor assembly since all ten (or in the small nodule, six) pods will simultaneously be brazed. The heavy pod collars perform the second vital load bearing function. The 60-atm helium pressure cannot be resisted by the walls of the gas distributor housing acting alone because of the large flat-plate area. Additional tension members are required as provided by the threaded pod collars. The central portion of the : od assembly is stressed only axially by the gas pressure. The outboard portions of the she11, $2 \mathrm{~mm}$ thick, experience the circumferential stress of any cylindrical pressure vessel plus the tension produced by the pressure on the hemispherical end cap. Inconel 718 has been chosen as the material for this entire structure. 
(Exploded view

- pods not shown)

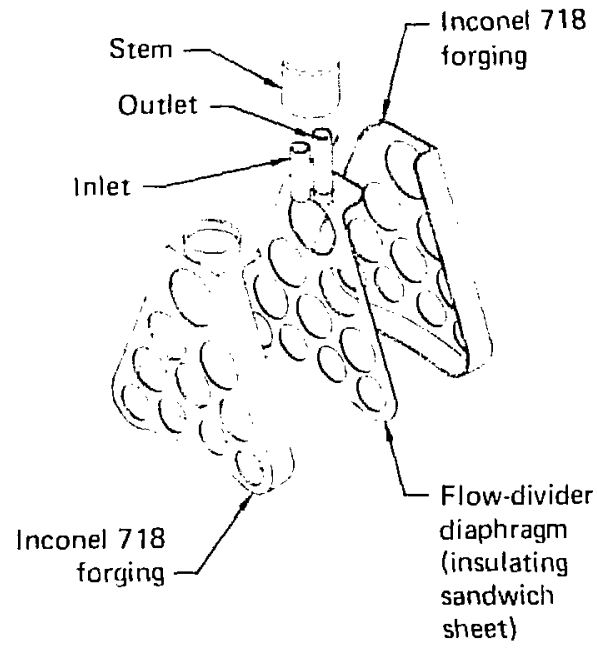

Fig. 8-6. Pod-module coolant flow distributor.

\section{BLANKET AND SHIELD NEUTRONICS*}

A Monte Carlo type code, TARTNP, is used extensively at LLL for neutronics calculations. Using that code, we ran both one-dimensional and three-dimensional problems describing our $\mathrm{Li}_{2} \mathrm{O}$ blanket concept. The geometry for the three-dimensional calculations is fairly complex. Only a few approximations were needed to permit reasonably short problem-running time.

Figure 8-7 shows a cross section of the blanket segment analyzed by the TARTNP three-dimensional runs. Adjacent segments are mirror images of the one illustrated and are so treated by "reflecting" regions described in the code input. Note that there are four annular rows of pod shells.

Whork performed by D. Hanchar, 1979 summer employee from Massachus.tts Institute of Technology, under the directior of J. D. Lee. 


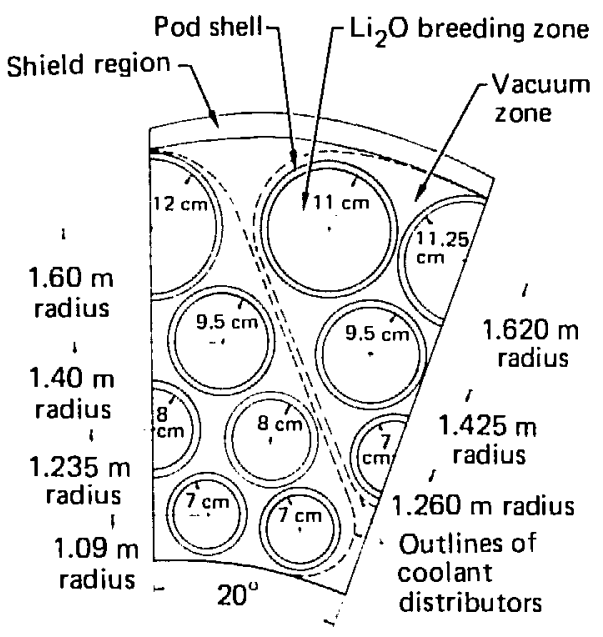

Fig. 8-7. End view of neutronics models.

The hemispherical ends on the pod shells were replaced by blunt (flat) cylinder ends. We bracketed the actual geometry with two cases: one, the cylinders extended from -100 to $+100 \mathrm{~cm}$ giving more than the actual volume of breeding $\mathrm{Li}_{2} \mathrm{O}$; and two, the cylinders were truncated at the plane where the radius of the largest hemishell begins. Figure 8-8 compares these two cases. Since Case 2 understates the actual volume of $\mathrm{Li}_{2} \mathrm{O}$ we are confident that the actual breeding ratio and blanket energy multiplication lie between the results of the two cases.

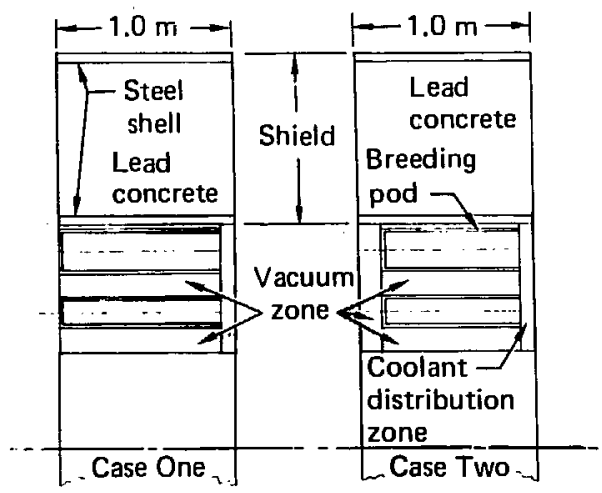

Fig. 8-8. Side view oi neutronics models. 
The reason only two pods appear in each part of Figure $8-8$ is that the pod-tubes are staggered. A radial plane cut through the blanket bisects the pods of alternate rows and misses pods in the remaining rows.

Both cases were run using various neutron batch sizes. Ten batches were run in all cases. The number of neutrons in each batch was taken as 400,600 and 800. Results are shown in Table 8-1 for the three assumed batch sizes. Tritium breeding ratios are shown for all three batch sizes. We present the energy multiplier for only the larger batch size.

TABLE 8-1. Blanket neutronics.

\begin{tabular}{ccccc} 
& $\frac{\text { Tritium breeding ratio }}{400} \frac{\text { Batch size }}{600}$ & 800 & $\frac{\text { Blanket energy multiplier }}{\frac{\text { Batch size }}{800}}$ \\
\hline $3 D^{a}$ Case 1 & 1.339 & 1.241 & 1.234 & 1.086 \\
3D Case 2 & 1.119 & 1.128 & 1.136 & 1.018 \\
\hline
\end{tabular}

a Three-dimensiona1

BLANKET AND SHIELD ASSEMBLY

In considering methods of reactor maintenance, the size and shape of the largest serviceable module is a difficult decision. We set a limit of 500 tonnes on module mass because nuclear components of that mass are routinely installed by conventional cranes. This limit imposed another limit of $\sim 2$ m on module length if a full cross-section "slice" of the reactor is to be removed. The solenoid coil shape and the desire to avoid conductor joints impose the geometry limit that any module must contain a full coil-hence, must be a "slice" of the entire centra! call assembly.

A result of the above decisions is that the parting planes between neighboring modules must pass between the spherical ends of adjacent pods. 
Also in that plane some clearance for removal must be provided. We provide a metal pressurized cushion in that region like a huge steel washer with the same inside diameter and outside diameter as the shield. It is only a few centimetres thick. Depressurizing it allows it to shrink and be slipped from between modules-just clearing the round noses of the pods. During operation, this seal-cushion provides a high impedance vacuum seal between modules. We believe cold-welding can be prevented. First, the contact pressure between the cushion face and the shield is only a few atmospheres. Second, a thin coating of molybdenum or other refractory alloy can be plasma-torchsprayed onto one of the surfaces to inhibit accidental bonding. No bolted or welded circumferential joints must be broken to removs one of the large modules. Figure 1-7 illustrates the arrangement of the module components. The seal cushion is discussed in more detail in a later section.

Each module has the gas-distributor stems of the pod-submodules on the module's center plane. There are two solenoidal coils on each module, symmetrically disposed from the center plane. The module sits in curved V-blocks surrounding the lower $180^{\circ}$ of the module. Those blocks take the unbalanced coil thrust (axial) which is zero or very small at the solenoid center and much larger near the two ends. The top half of the coils must also be supported, and several wedge blocks will be provided, oriented for vertical removal by the crane.

Gas manifolds for the helium coolant are somewhat unique and are shown in Fig. 8-9. To conserve space at the bottom of the machine one master manifold serves two adjacent modules. Upon reaching the horizontal centerline this manifold bifurcates and becomes two parallel manifolds on opposite sides of the module division plane. These two smaller manifolds lift off the large fiswer manifolds with the module when it is being removed by the crane. This requires that the pod stem feed pipes for the lower ha1f of the module have 
remote-disconnect joints so they may be spparated from the lower fixed manifold. Obviously there must also be four manifold couplings to permit division of the two smaller manifolds near the horizontal centerline.

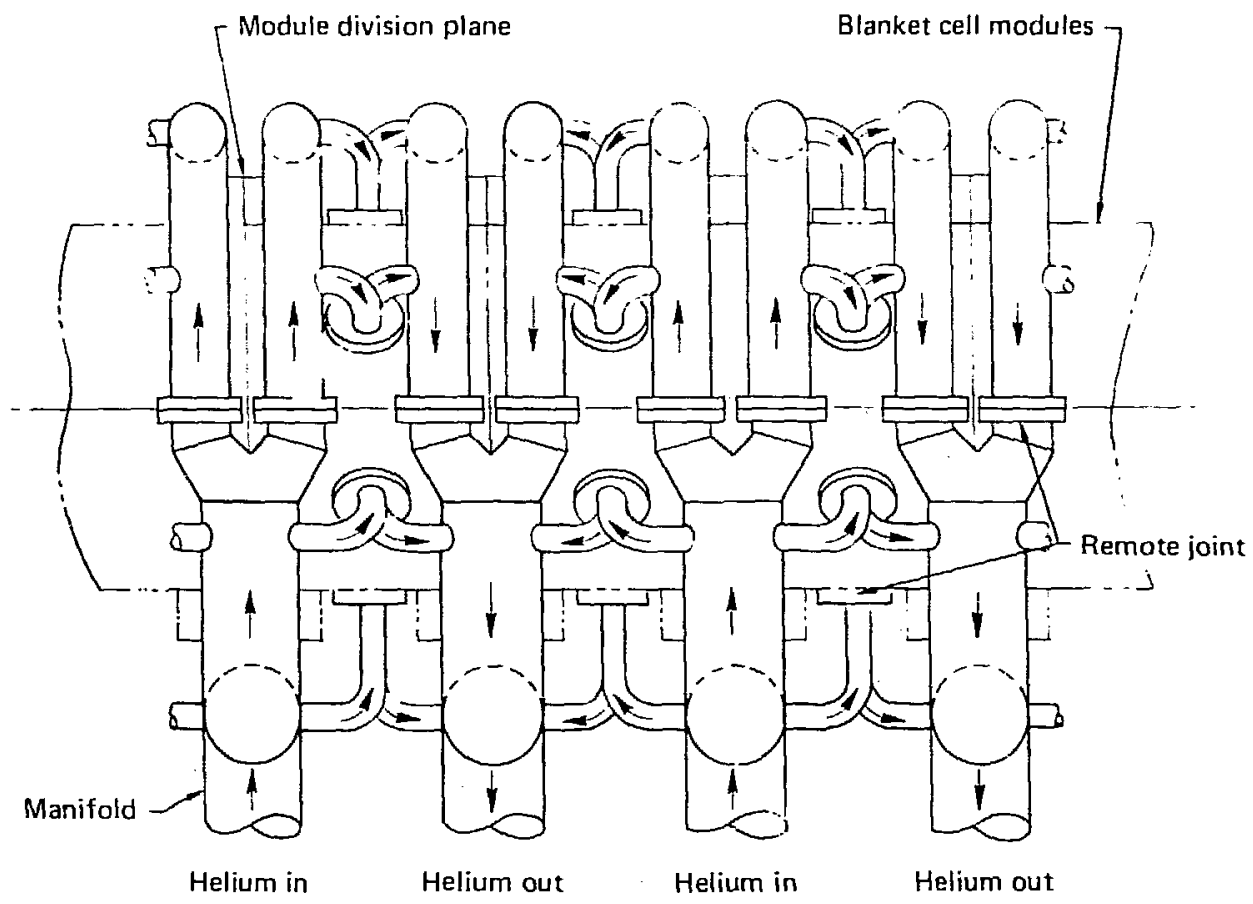

Fig. 8-9. Coolant gas manifold system.

There will be twenty helium pipe joints to open by zemote tools whe.: a module is removed: four $20 \mathrm{~cm}$ in diameter (on manifolds) and sixteen $6 \mathrm{~cm}$ in diameter (two on each pod stem connection). 
We reduce fabrication costs of the central cell by using a very inexpensive shield and integrating the magnet fabrication with the shield so that duplication of form and structure can be avoided. The shield is fabricated as a hollow shell-an inner cylindrical shell, two face-plate "washers," and an outer shell. The outer shell of the shield cylinder is used as the coil form for the solenoid magnets. Appropriate layers of insulation would be included between the shield outer wall and the superconductor. The dimensions of the shield for one central ce11 module are $2.2 \mathrm{~m}$ inner radius, $1.0 \mathrm{~m}$, radial thickness, and $2 \mathrm{~m}$, length. After coil winding and installation, the hollow snell would then be poured full of lead-concrete. This "concrete" uses lead shot as the aggregate. A range of sizes will yield a higher packing factor. A high density matrix material $(\rho=10 \mathrm{~g} / \mathrm{cm})$ is used to fill the voids and complete the lead shield without pouring molten lead. This matrix materal is proprietary with Chemtree Corp., Centra1 Va11ey, New York, and is called "Lead-cement."

\section{Sea1 Washer}

The pressurized-cushion sea $1^{*}$ between central ce11 modules was mentioned earlier in the section, Blanket and Shield Assembly. It must provide a very low conductance gas seal between the $10^{-2}$-Torr vacuum, which can be readily achieved in the "trench" surrounding the central cell, and the plasma chamber

झPatent applied for by General Atomic Company, San Diego, as a result of 1978-1979 contract work with Lawrence Livermore Laboratory and Pacific Gas and Electric Company for the Electric Power Research Institute. 
pressure which must be in the $10^{-6}$ to $10^{-7}$ Torr range. This low conductance is achieved by flat plate contact over a radial dimension of $1 \mathrm{~m}$ (the shield thickness) with an assumed average gap of $0.03 \mathrm{~mm}$. The conductance of such a gap is about $0.04 \mathrm{litres} / \mathrm{s}$. With 28 modules (hence 58 sealing surFaces) the total gas inflow is 0.02 Torr-litres/s, a very small gas load. A method of halving that gas flow is to build the cushion as a permanent part of the shield on one end of each module. This method has the added advantage of not requiring a separate disassembly operation to remove the seal cushion. A disadvantage is the reduced module installation clearance.

The cushion seal, whether separable or integrated with the shield, uses amega-joint expansion elements, which behave similarly to a bellows but are much more rugged. The price of ruggedness is comparative stiffness. Only 1 cm of clearance for assembly can be obtained by evacuating the cushion to allow the ambient pressure in the trench to collapse it. Though normally evacuated, the trench is backfilled to atmospheric pressure with dry ait or inert gas prior to the beginning of a module change-over.

Another concern for the cushion seal is neutron leakage through the gap between adjacent shield segments. This gap need not be unimpeded radially. The two faces of the cushion can be contoured on their inner surfaces (in contact with the pressure energizing fluid) to create a labyrinth (see Figure 8-10). Some neutron leakage is unavoidable because the effective shield thickness will be reduced in the cushion zone; however, the leaking neutrons are not aimed at a solenoid coil. The upper half of the machine, where such leakage has biological significance, can have fitted shield pieces separately lifted away by the crane before module removal. The lower half of the leakage goes into the concrete foundation, service gallery hardware, and the earth. 


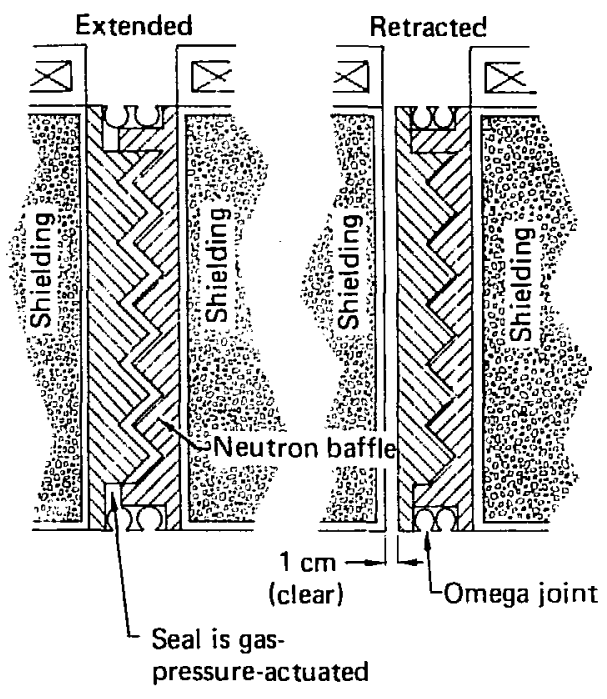

Fig. 8-10. Madule-to-module seal cushion.

CENTRAL CELL SERVICING

Two methods of blanket replacement are available to operators of this TMR: (1) use of the gantry crane to remove a 2-m-lang central-cell module containing 18 pod-submodules, or (2) using a service car operating in cooperation with disconnect tools, removal of only the pod-submodule which has experienced a failure. The first method should be used for periodic first-wall replacement. The second method applies to unscheduled blanket repairs.

Large Module Replacement

The design feature of this reactor, which permits rapid change of central cell modules, is the vacuum trench surrounding it. This trench contains locators and supports for each module and a number of hinged covers, one or two of which have to be lifted to yield access to any given module. The vacuum seals of the trench are all static. The cover seals must be opened and 
resealed several times in the 1 ife of the reactor. The seals can be elastomeric since they are in a low radiation environment and are easily replaced. Along the short edge of the cover the vacuum seal is between the cover and the building structure. Along the long edge the seal is between adjacent covers. The corner of the cover presents a difficult topological problem. We suggest an inflatable hose design of square cross section to provide seal effectiveness at the corners. Figure 8-11 shows the transition region at the corner. The procedure for removal of a large module is outlined below:

1. Shut off plasma by stopping all neutral beam injection, de-energize magnets .

2. Backfill vacuum trench with dry air or inert gas.

3. Deflate all door seals.

4. Using the traveling crane, remove the desired trench $\operatorname{cover}(s)$ and store them.

5. Deflate all pressurized seal cushions between modules and evacuate the cushion cavity of those seals affecting the desired module.

6. Lift out magnet thrust blocks and store.

7. Disconnet cryogenic services to solenoid coils on desired module.

8. Disconnect instrumentation on module.

9. Disconnect helium purge lines for tritium recovery.

10. Disconnect manifold feeders between fixed lower helium manifold and eight lower pod-module subassemblies (sixteen remotely operated couplings).

11. Disconnect four remotely operated couplings that attach the upper helium manifolds to the fixed lower manifolds.

12. Attach the crane hoist to the module and lift the module assembly out of the trench; translate the module to a waiting transporter in the service space beside the trench. 


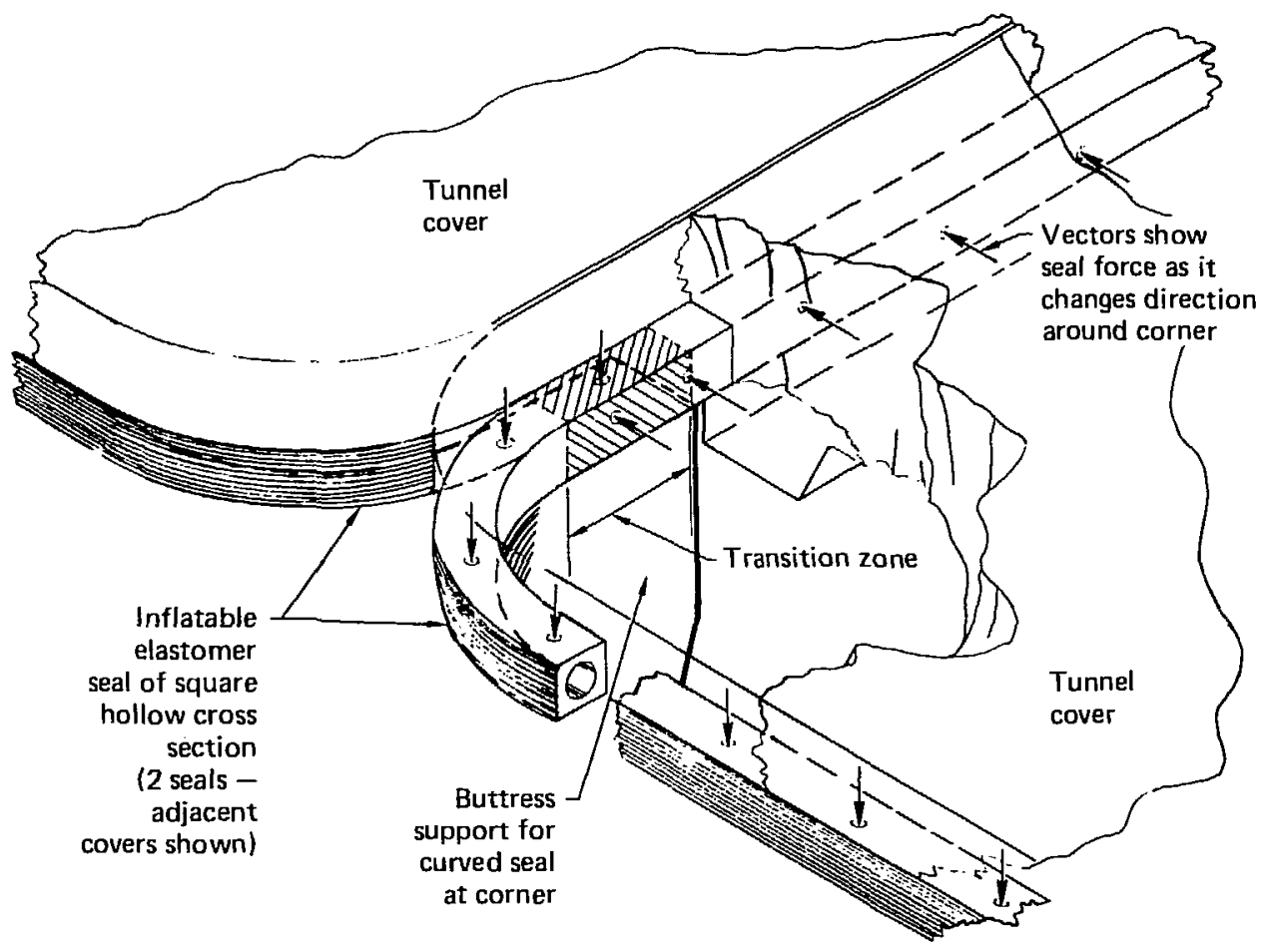

Fig. 8-11. Vacuum trench cover seal.

13. Mount the module on the transporter.

14. Transport to the hot cell for module disassembly.

15. Using a new-module transporter, bring a new pretested module from the assembly hall to a position next to the open trench cover.

16. Install the replacement module reversing the procedure outlined above.

As many modules as desired can be lifted out serially to accomplish replacement of a portion of the first-wall. Items 6 through 11 (above) can be performed simultaneously on all modules to be replaced thereby shortening multiple module change-over time. 
If a $\mathrm{flaw}$ is detected in one pod-submodule its replacement can be accomplished more quickly by another service scenario. At the two ends of the central cell are solenoidal coils generating a 12-T magnetic field between the central cell and the thermal barrier region. These barrier coils, by their physical presence, prevent large objects from entering the "bore" of the central cell. No other reactor component interferes with translation of these coils perpendicular to the reactor axis to temporarily eliminate this obstruction. The sections of the reactor in which these coils reside can be part of two tunnels running perpendicular to the reactor axis and containing a vacuum-airlock separating the reartor from a hot cell. The tunnels are shown in Fig. 8-12 (it will be noted that the "dead end" of each tunnel which extends several meters past the reactor serves as a parking place for the barrier coil).

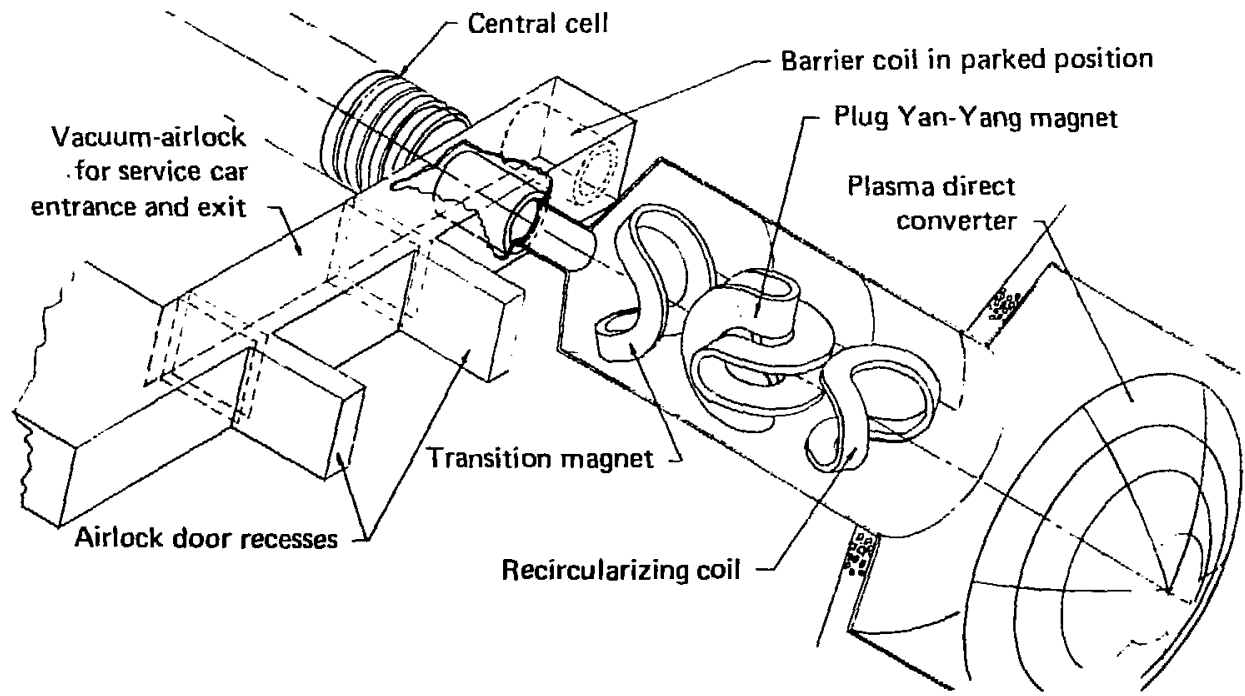

Fig. 8-12. Service-car access tunnel. 
The service $c a r(s)$ park in the vacuum-airlock under normal operating conditions. When blanket service is required the barrier coil is disconnected from its current and cryogenic connections and moved aside to its parking area. The "movers" are large pneumatic cylinders and the transport tracks are below the coil and well clear of the plasma zone. Some bracing structure that serves to hold the coil in place against axial magnetic forces will have to be disengaged to permit the coil's movement.

A concept of the service car is shown in Fig. 8-13. It is equipped with carbon-cloth-covered pneumatic tires mounted on bogie trucks. The wheels roll directly on the pods and the coolant distributor housings. The bogie trucks smooth the "ride" for the module being transported. No special tracks are necessary. (Tracks would have to be cooled if permanently built-in, or would be time-consuming to install if temporary.) The blanket submodule can be replaced as follows:

1. Shut off plasma by stopping all neutral beam injection.

2. Relocate the barrier-cell mirror coil as described previously.

3. Bring the service car to the centerline of the reactor through the vacuum-airlock in the tunnel; the car moves sideways on a transporter to get into position at the open end of the central cell.

4. Move car to desired position in the central cell using laser quadcell control to steer and to position the car.

5. Extend positioning dowels to accurately aiign the car.

6. Extend the rotating inner she11 of the car and rotate it to the desired angle.

7. Engage pod-module grippers to the module desired. 


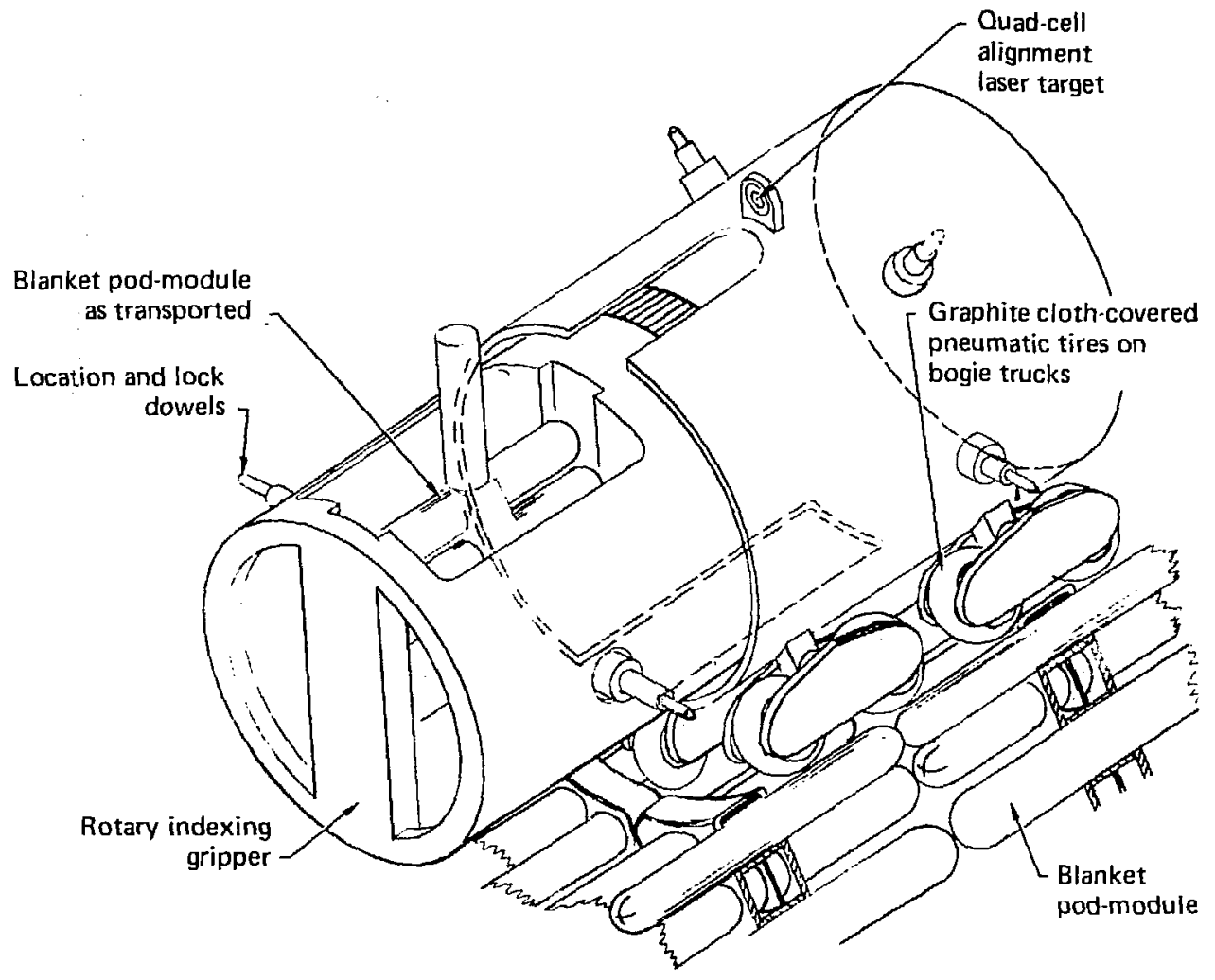

Fig. 8-13. B1anket service car.

8. By using remote tools located in the space provided outside the shield and between the two coils, remove two helium pipe connections and unbolt the gas system cover plate; park the plate nearby in the service gallery.

9. Grind off the pod stem weld that attaches the stem to the back of the shield.

10. Withdraw the pod-module into the service car extension. 
11. Retract the service-car extension cylinder back into the car (with the pod-module inside).

12. Move the car back to the entrance point and onto the transporter.

13. Transport the car with the faulty pod-module to a hot cell that is part of the tunnel system.

14. Load a new pod into the service car.

15. Repeat the previous steps for insta11ation of the replacement pod.

SUMMARY OF CENTRAL CELL DESIGN AND COST ESTIMATE

Our new centra1 ce11 has the following design features:

- Efficient cylindrica1 pod pressure vesse1s for the blanket

- $\mathrm{Li}_{2} \mathrm{O}$ neutron moderator: thin blanket

- Helium coolant

- One-tonne replaceable blanket pod-modules

- Access to pod connections Irom outside the shield

- Poured lead-cement shield

- Combined coil winding form and shield case

- A choice of maintenance methods:

a. Service car in bore of central ce11 for unscheduled blanket maintenance on pod-modules

b. Large-module ehange-out for scheduled first-wall replacement (a 2-m-thick "slice" of the central cell)

Our first TMR design (1977) had fabrication costs fo the centra1 cell of $3.0 \mathrm{million}$ dollars/m of length. As shown in Table 8-2 this new design will total about 1.72 million dollars/m. 
TABLE 8-2. Cost estimate of TMR central cell.

\begin{tabular}{|c|c|c|}
\hline Item & Description & $\begin{array}{c}\text { Cost } \\
\text { (million \$) }\end{array}$ \\
\hline T-breeder & $\mathrm{Li}_{2} \mathrm{O}$ He cooled & 0.41 \\
\hline Blanket structure & $\begin{array}{l}\text { Inconel } 718 \text { tube } \\
\text { and forge } \$ 35 / \mathrm{kG}\end{array}$ & 0.15 \\
\hline Shield & - & 0.66 \\
\hline $\begin{array}{l}\text { Solenoid coil superconductor } \\
\text { and coil structure }\end{array}$ & $\begin{array}{l}\$ 22 / \mathrm{kG} \\
\$ 15 / \mathrm{kG} \text { structure }\end{array}$ & 0.50 \\
\hline Vacuum cylinder & Part of shield & - \\
\hline \multirow[t]{2}{*}{ Main support structure } & Part of building & - \\
\hline & 1 cost per meter & 1.72 \\
\hline
\end{tabular}




\section{REFERENCES}

8-1. W. S. Neef, Jr., Mechanical Design Aspects of a Tandem Mirror Fusion Reactor, Lawrence Livermore Laboratory, Livermore, CA, UCRL-79434 (1977).

8-2. D. J. Bender, R. S. Devoto, J. H. Fink, T. R. Galloway, W. E. Kastenberg, J. D. Lee, W. S. Neef, Jr., K. R. Schultz, D. W. Culber, S. B. Rao, and S. R. Rao, Reference Design for the Standard Mirror Hybrid Reactor, Lawrence Livermore Laboratory, Livermore, CA, UCRL-52478 (1978).

8-3. K. Tanaka, H. Kudo, and H. Amano, "Tritium Recovery of the Fusion Reactor Lithium Oxide Blanket," Proc. Int. Conf. on Radiation Effects and Tritium Technology for Fusion Reactors, Gattlinburg, TN, October 1-3, 1975, CONF-7509893253.

8-4. S. Nasu, H. Kudo, K. Shiozawa, T. Takahashi, T. Kurasawa, M. Tachiki, and K. Tanaka, "Tritium Release From Neutron Irradiated Li ${ }_{2} \mathrm{O}^{\text {Pellets," }}$ J. Nuc. Mater., 68261 (i977). 